ESCOLA POLITÉCNICA DA UNIVERSIDADE DE SÃO PAULO DEPARTAMENTO DE ENGENHARIA DE SISTEMAS ELETRÔNICOS

\title{
REDE DE SENSORES PARA ENGENHARIA BIOMÉDICA UTILIZANDO O PROTOCOLO IEEE1451
}

WESLEY BECARI 
WESLEY BECARI

\section{REDE DE SENSORES PARA ENGENHARIA BIOMÉDICA UTILIZANDO O PROTOCOLO IEEE1451}

Dissertação apresentada à Escola Politécnica da Universidade de São Paulo para obtenção do Título de Mestre em Engenharia.

Área de Concentração: Sistemas Eletrônicos

Orientador: Prof. Dr. Francisco Javier Ramirez-Fernandez 
AUTORIZO A REPRODUÇÃO E DIVULGAÇÃO TOTAL OU PARCIAL DESTE TRABALHO, POR QUALQUER MEIO CONVENCIONAL OU ELETRÔNICO, PARA FINS DE ESTUDO E PESQUISA, DESDE QUE CITADA A FONTE.

Este exemplar foi revisado e alterado em relação à versão original, sob responsabilidade única do autor e com a anuência de seu orientador.

São Paulo, 2 de fevereiro de 2012.

Assinatura do autor

Assinatura do orientador

\section{FICHA CATALOGRÁFICA}

Becari, Wesley

Rede de sensores para engenharia biomédica utilizando o protocolo

IEEE1451 / W. Becari. -- ed.rev. -- São Paulo, 2012.

$184 \mathrm{p}$.

Dissertação (Mestrado) - Escola Politécnica da Universidade de São

Paulo. Departamento de Engenharia de Sistemas Eletrônicos.

1. Sensores biomédicos 2. Bioengenharia 3. Rede de sensores

I. Universidade de São Paulo. Escola Politécnica. Departamento de Engenharia de Sistemas Eletrônicos. II. t. 


\section{DEDICATÓRIA}

Dedico, nas pessoas de meus pais, a todos aqueles que se esforçam para educar a juventude. 


\section{AGRADECIMENTOS}

Em primeiro lugar ressalto a grande estima que possuo pelo professor Francisco Javier Ramirez-Fernandez. Iniciei o trabalho no grupo de Sensores Integráveis e Microssistemas (gSIM) no primeiro ano de graduação da Escola Politécnica da Universidade de São Paulo. Desde então percorro um caminho de aprendizagens, vivências e experiências. E justamente através dessas experiências percebi no professor Javier uma qualidade de poucos, sua personalidade. Retomo aqui uma passagem do livro do Apocalipse, capítulo 3, versículos 15 e 16 que diz: “Conheço tuas obras: não és frio nem quente. Oxalá fosses frio ou quente! Mas, porque és morno, nem frio nem quente, estou para vomitar-te de minha boca”. Dessa passagem fica marcado que Deus vomitará os mornos. Nesse sentido, posso afirmar que o professor Javier nunca foi, não é e nunca será uma pessoa morna, que vive o seguro, o apático. E é de pessoas quentes e íntegras, como o professor Javier, que precisamos.

À Andréia, mulher amada, pelo seu carinho. À Maria Clara, minha filha, por toda a alegria do mundo. Aos meus pais, Heloísa e Waldir, bem como aos meus irmãos, Willy e Christiane, e minha avó Luiza, por todo apoio, vivência e afeto.

Cabe ressaltar o apoio dos amigos Henrique, Maurício, Jair, Fátima, Márcio, Luqueze, Latife, Sandro e Bete, que dividem comigo as alegrias e angústias do trabalho na Universidade de São Paulo. Aos amigos de graduação Fernando, Murilo, Felipe, Vitor, Mirele, Gleison, Rhemzo e Vanessa.

À Capes pelo apoio financeiro durante o primeiro ano de trabalho. 


\section{RESUMO}

A utilização de sensores e de atuadores cresceu vertiginosamente nos últimos anos. As aplicações centralizadas em sensoriamento e controle avançaram com a instrumentação industrial, passando pela incorporação desses elementos em redes distribuídas até culminar, na atualidade, em redes integradas que possuem inúmeras funções e aplicações, dentre elas: controle, monitoramento, rastreamento e segurança. Entretanto o crescimento do número de sensores e atuadores conectados através de barramentos e redes não ocorreu de forma única, proliferando uma diversidade de formas de padronização na comunicação entre esses e seus respectivos monitores ou controladores. Dessa pluralidade de protocolos emergiu a necessidade de criação de um padrão que permitisse interoperabilidade entre transdutores e redes de controle, bem como a introdução do conceito de sensores e atuadores inteligentes. Nesse contexto foi proposto o protocolo IEEE1451 (Standards for Smart Transducer Interface for Sensors and Actuators). Nessa perspectiva o trabalho em questão apresenta os resultados do desenvolvimento e a utilização desse padrão em duas aplicações de engenharia biomédica. Primeiramente em um sistema embarcado capaz de realizar aquisição e processamento de biopotenciais, em específico, de eletrocardiogramas e a posterior transmissão dos dados coletados através de uma interface sem fio Wifi (IEEE802.11b/g) utilizando os protocolos IEEE1451.0 e IEEE1451.5. E em um eletrogoniômetro, para fins fisioterápicos, que realiza medidas de amplitude de movimento das articulações e transfere os dados serialmente através de uma interface USB (Universal Serial Bus) utilizando como padrões o IEEE1451.0 e o IEEE1451.2. Ambos os sistemas permitem além do monitoramento local, o acesso das informações coletadas pela Internet através de uma instrumentação remota implementada por um Web Service. Dessa maneira foi construída uma rede de sensores padronizada que permitiu demonstrar os benefícios e as potencialidades do protocolo IEEE1451.

Palavras Chaves: Rede de Sensores. Transdutores Inteligentes. Protocolo IEEE1451. Engenharia Biomédica. Bioengenharia. Sensores Médicos. 


\begin{abstract}
The use of sensors and actuators grew vertiginously in recent years. The centralized applications in sensing and controlling advanced with industrial instrumentation, going through the incorporation of these elements into industrial computer network protocols until currently culminating in integrated networks with innumerable functions and applications, amongst them: controlling, monitoring, tracking and security. However, the growth of the number of sensors and actuators connected through bus and computers networks did not occur in a single way, and a diversity of communication standards proliferated. From these protocols, plurality required the creation of a standard to allow interoperability between transducers and networks, as well as the introduction of the smart sensors and actuators concept. In this context the IEEE1451 protocol (Standards for Smart Transducer Interface for Sensors and Actuators) was proposed. In this perspective, this work presents two applications of biomedical engineering using the IEEE1451 standard. The first application is an embedded system for biopotencial acquisition and processing, specifically, electrocardiogram signals and data transmission through a wireless interface Wifi (IEEE802.11b/g) using IEEE1451.0 and IEEE1451.5 protocols. The other application is an electrogoniometer, for physiotherapy, that measures the joint movements amplitude and transfers the data through an USB (Universal Serial Bus) connection using the IEEE1451.0 and the IEEE1451.2 protocols as standards. Both systems allow local monitoring and the access to information through a remote instrumentation implemented by Web Services. Hence, this project demonstrates the IEEE1451 benefits and the potentialities.
\end{abstract}

Keywords: Sensors Networks. Smart Transducers. IEEE1451. Biomedical Engineering. Bioengineering. Medical Sensors. 


\section{LISTA DE ILUSTRAÇÕES}

Figura 2.1 - Modelo de Transdutor Inteligente (SONG; LEE, 2008a)..................... 27

Figura 2.2 - Modelo de Transdutor Inteligente utilizando o protocolo IEEE1451 (SONG; LEE, 2008a).................................................................. 30

Figura 2.3 - Estrutura do Protocolo IEEE1451 (SONG; LEE, 2007). ...................... 31

Figura 2.4 - Arquitetura da norma IEEE1451.0 .................................................. 33

Figura 2.5 - Arquitetura da norma IEEE1451.2 _.............................................. 37

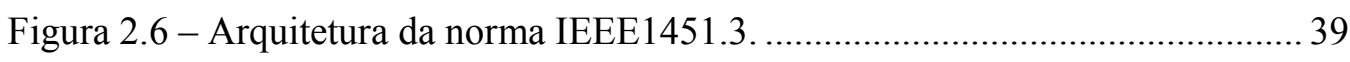

Figura 2.7 - Estrutura da norma IEEE1451.4 .................................................... 40

Figura 2.8 - Modelo de aplicação de Web Services. Adaptado de Gomes (2009). ... 46

Figura 2.9 - Estrutura em camadas de um Web Service ......................................... 48

Figura 2.10 - Detalhe das câmaras do coração. Adaptado de Guyton e Hall (2006). 53

Figura 2.11 - Eletrocardiograma normal. Adaptado de Guyton e Hall (2006).......... 55

Figura 2.12 - Derivações eletrocardiográficas (IVLINE, 2011)............................. 57

Figura 2.13 - Movimentos realizados pelo ombro. Adaptado de Lippert (2006)...... 59

Figura 2.14 - Movimentos realizados pelo cotovelo. Adaptado de Lippert (2006). . 60

Figura 2.15 - Movimentos realizados pelo punho. Adaptado de Lippert (2006)..... 61

Figura 3.1 - Arquitetura da rede de sensores biomédicos utilizando o protocolo

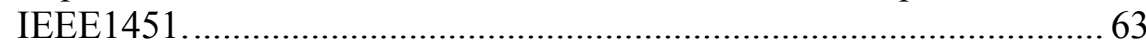

Figura 3.2 - Esquema de circuitos para condicionamento de biopotenciais.............. 66

Figura 3.3 - Esquema do filtro Butterworth passa-altas de $2^{\mathrm{a}}$ ordem........................67 67

Figura 3.4 - Esquema do filtro notch duplo T rejeita-faixas. ................................... 68

Figura 3.5 - Esquema do filtro Butterworth passa-baixas de $4^{\mathrm{a}}$ ordem...................... 69

Figura 3.6 - Esquema dos estágios amplificadores não inversores e somadores. ..... 71

Figura 3.7 - Esquema de funcionamento do acelerômetro a gás (MEMSIC, 2011). 73

Figura 3.8 - Medida da inclinação das articulações utilizando acelerômetros.......... 74

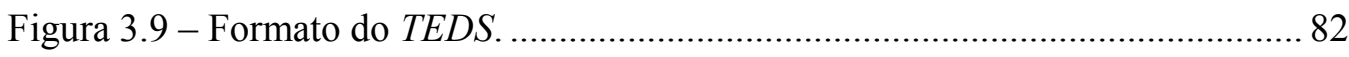

Figura 3.10 - TEDS do IEEE1451.0 (SONG; LEE, 2008) .................................... 84

Figura 3.11 - Modelo de referência IEEE1451.0 .................................................... 93

Figura 3.12 - Conexão direta entre WTIM e NCAP ............................................... 98

Figura 3.13 - Arquitetura de acesso remoto através de Web Services.................... 101

Figura 4.1 - Esquema elétrico do filtro passa-altas de $2^{\mathrm{a}}$ ordem. ............................ 104 
Figura 4.2 - Curva de resposta em frequência do ganho e fase do filtro passa-altas de $2^{\mathrm{a}}$ ordem. 105

Figura 4.3 - Esquema elétrico do filtro rejeita-faixas. 105

Figura 4.4 - Curva de resposta em frequência do ganho e fase do filtro rejeita-faixas. 106

Figura 4.5 - Esquema elétrico do filtro passa-baixas de $4^{\mathrm{a}}$ ordem. 107

Figura 4.6 - Curva de resposta em frequência do ganho e fase do filtro passa-baixas. 107

Figura 4.7 - Curva de resposta em frequência do ganho e fase do circuito condicionador de biopotenciais. 108

Figura 4.8 - Protótipo do circuito de condicionamento de biopotenciais. 109

Figura 4.9 - Resposta em frequência do circuito condicionador de biopotenciais. . 110 Figura 4.10 - Sinal de ECG condicionado. 111

Figura 4.11 - Medida de inclinação com o MX2125 em três inclinações distintas. 113 Figura 4.12 - Dois acelerômetros MX2125 alocados no braço e antebraço. 118

Figura 4.13 - Sistema embarcado microcontrolado para desenvolvimento do STIM. 121

Figura 4.14 - Sistema embarcado microcontrolado para desenvolvimento do WTIM.

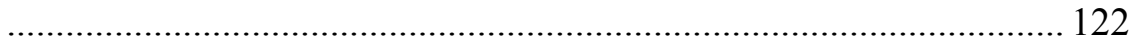

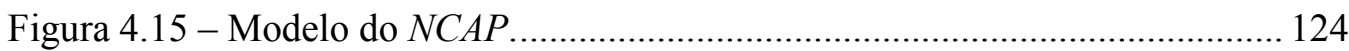

Figura 4.16 - Interface do software NCAP desenvolvido..................................... 124

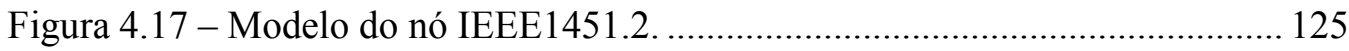

Figura 4.18 - Diagrama sequencial de conexão entre o TIM e o NCAP .................. 126

Figura 4.19 - Acesso às informações do STIM ........................................................ 127

Figura 4.20 - Acesso às informações dos TEDS do STIM...................................... 128

Figura 4.21 - Disponibilização dos dados coletados no sensor do STIM................ 128

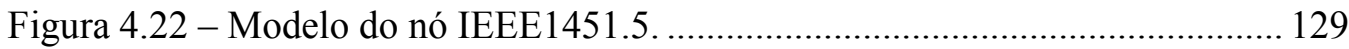

Figura 4.23 - Acesso às informações do WTIM. ................................................... 130

Figura 4.24 - Acesso às informações dos TEDS do WTIM. ................................... 131

Figura 4.25 - Disponibilização dos dados coletados no sensor do WTIM............... 131

Figura 4.26 - Descrição WSDL do Web Service desenvolvido................................ 132

Figura 4.27 - Resposta do NCAP à requisição de dados do STIM. ......................... 133

Figura 4.28 - Resposta do NCAP à requisição de dados do WTIM......................... 134

Figura 4.29 - Interface inicial do framework NIST IEEE1451.0 e IEEE1451.5 .... 135

Figura 4.30 - Anúncio do PHY TEDS do WTIM. ................................................... 136

Figura 4.31 - Recebimento da informação do TEDS do WTIM. ............................. 137 
Figura 4.32 - Transmissão dos dados coletados pelo medidor de frequência cardíaca.

138

Figura 4.33 - Rede de sensores com STIM, WTIM e NCAP .................................... 140 


\section{LISTA DE TABELAS}

Tabela 2.1 - Mapeamento entre elementos WSDL e elementos UDDI. .................... 52

Tabela 3.1 - Magnitudes de biopotenciais (WEBSTER, 1998)................................ 64

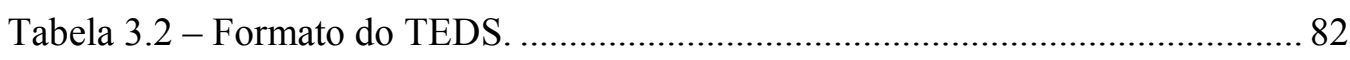

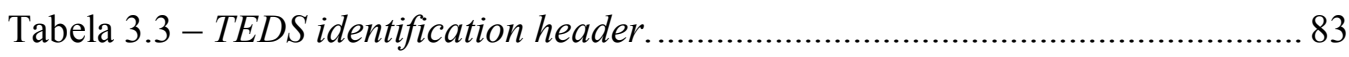

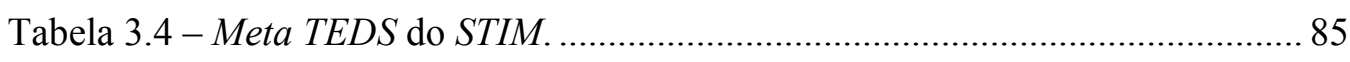

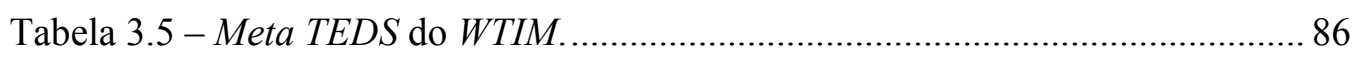

Tabela 3.6 - TransducerChannel TEDS do STIM. ................................................ 87

Tabela 3.7 - TransducerChannel TEDS do WTIM............................................... 88

Tabela 3.8 - User's Transducers Name TEDS do STIM. ....................................... 91

Tabela 3.9 - User's Transducers Name TEDS do WTIM....................................... 92

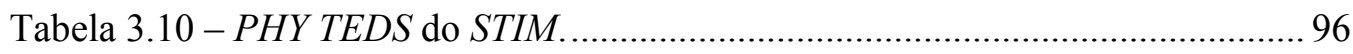

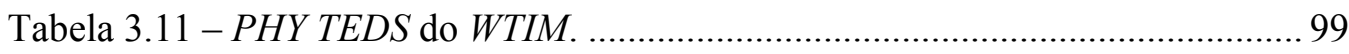

Tabela A.1 - Caracterização do eixo x do acelerômetro MX2125 …...................... 170

Tabela A.2 - Caracterização do eixo y do acelerômetro MX2125 …...................... 171

Tabela A.3 - Caracterização do acelerômetro MX2125 ......................................... 172

Tabela A.4 - Caracterização do MX2125 para medida de ADM........................... 173

Tabela A.5 - Meta TEDS IEEE1451.0 (IEEE, 2007a). ........................................ 180

Tabela A.6 - TransducerChannel TEDS IEEE1451.0 (IEEE, 2007a).................... 181

Tabela A.7 - User's Transducer Name TEDS IEEE1451.0 (IEEE, 2007a)........... 182

Tabela A.8 - PHY TEDS IEEE1451.2 extraído de Song e Lee (2008b).................. 183

Tabela A.9 - PHY TEDS IEEE1451.2 adaptado de IEEE P1451.2TM/D21 (não

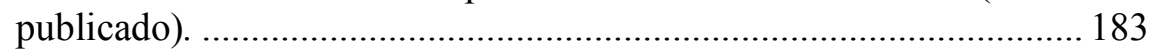

Tabela A.10 - PHY TEDS IEEE1451.5 (IEEE, 2007b).................................... 184 


\section{LISTA DE GRÁFICOS}

Gráfico 4.1 - Caracterização de um acelerômetro como inclinômetro..................... 117 Gráfico 4.2 - Relação entre ângulo medido pelo acelerômetro e ângulo de referência. 


\section{LISTA DE ABREVIATURAS}

AC Alternative Current

ADM Amplitude de Movimento

APIs Application Programming Interfaces

ASCII American Standard Code for Information Interchange

ASP Active Server Pages

AV Nódulo Atrioventricular

AXIS Apache EXtensible Interaction System

BACNet Building Automation Control Network

CAN Controller Area Network

CDC Communication Device Class

CEbus Consumer Electronics Bus

CI Circuito Integrado

CLP Controlador Lógico Programável

CMOS Complementary Metal-Oxide-Semiconductor

CMRR Common-Mode Rejection Ratio

CORBA Common Object Request Broker Architecture

CPLD Complex Programmable Logic Device

DC Direct Current

DCOM Distributed Component Object Model

DSP Processador Digital de Sinais

ECG Eletrocardiografia

EEG Eletroencefalografia

EMG Eletromiografia

ENG Eletroneurograma

ERG Eletroretinograma

FPGA Field-Programmable Gate Array

FIR Finite Impulse Response

FTP File Transfer Protocol

GND Ground 


\begin{tabular}{|c|c|}
\hline GPRS & General Packet Radio Service \\
\hline GPS & Global Positioning System \\
\hline GUI & Graphical User Interface \\
\hline HID & Human Interface Device \\
\hline HL7 & Health Level 7 \\
\hline HTTP & Hypertext Transfer Protocol \\
\hline $\mathbf{I} / \mathbf{O}$ & Input/Output \\
\hline IEEE & Institute of Electrical and Electronics Engineers \\
\hline IIR & Infinite Impulse Response \\
\hline IP & Internet Protocol \\
\hline ISM & Industrial Scientific and Medical \\
\hline JDDAC & Java Distributed Data Acquisition and Control \\
\hline JDK & Java Development Kit \\
\hline JSP & JavaServer Pages \\
\hline LIN-Bus & Local Interconnect Network \\
\hline LNA & Low Noise Amplifier \\
\hline LUFA & Lightweight USB Framework for AVRs \\
\hline MEMS & Micro-Eletro-Mecânicos \\
\hline MIPS & Milhões de instruções por segundo \\
\hline MMI & Mixed Mode Interface \\
\hline NCAP & Network Capable Application Processor \\
\hline NIST & National Institute of Standards and Technology \\
\hline PA & Power Amplifier \\
\hline PCs & Personal Computers \\
\hline PDA & Personal Digital Assistant \\
\hline PHP & Hypertext Preprocessor \\
\hline Profibus & Process Field Bus \\
\hline $\mathbf{R A M}$ & Random Access Memory \\
\hline RFID & Radio-Frequency IDentification \\
\hline RISC & Reduced Instruction Set Computing \\
\hline RMI & Remote Method Invocation \\
\hline RPC & Remote Procedure Call \\
\hline
\end{tabular}




\begin{tabular}{|c|c|}
\hline RS-232 & Recommended Standard 232 \\
\hline RS-485 & Recommended Standard 485 \\
\hline SA & Sinoatrial \\
\hline SMTP & Simple Mail Transport Protocol \\
\hline SOA & Service-Oriented Architecture \\
\hline SOAP & Simple Object Access Protocol \\
\hline SPI & Serial Peripheral Interface Bus \\
\hline SPICE & Simulation Program with Integrated Circuit Emphasis \\
\hline SSID & Service Set Identifier \\
\hline STIM & Smart Transducers Interface Module \\
\hline TBC & Transducer Bus Controller \\
\hline TBIM & Transducer Bus Interface Module \\
\hline TCP & Transmission Control Protocol \\
\hline TEDS & Transducer Electronic Data Sheet \\
\hline TII & Transducer Independent Interface \\
\hline TIM & Transducer Interface Module \\
\hline TLV & Type/Length/Value \\
\hline UART & Universal Asynchronous Receiver/Transmitter \\
\hline UDDI & Universal Description Discovery and Integration \\
\hline UDP & User Datagram Protocol \\
\hline URI & Uniform Resource Identifier \\
\hline UML & Unified Modeling Language \\
\hline URL & Uniform Resource Locator \\
\hline USB & Universal Serial Bus \\
\hline UUID & Universal Unique Identifier \\
\hline UWB & Ultra-Wide Band \\
\hline W3C & World Wide Web Consortium \\
\hline WSDD & Web Services Deployment Descriptor \\
\hline WSDL & Web Services Description Language \\
\hline WWW & World Wide Web \\
\hline XML & Extensible Markup Language \\
\hline WTIM & Wireless Transducer Interface Module \\
\hline
\end{tabular}




\section{LISTA DE SÍMBOLOS}

$\begin{array}{ll}\mathbf{A} & \text { Ampere } \\ \mathbf{b p m} & \text { Batimento por minuto } \\ \mathbf{d B} & \text { Decibel } \\ \mathbf{F} & \text { Faraday } \\ \mathbf{g} & \text { Unidade de aceleração } \\ \mathbf{H} & \text { Henry } \\ \mathbf{H z} & \text { Hertz } \\ \mathbf{o} & \text { Grau } \\ \mathbf{V} & \text { Volt } \\ \boldsymbol{\sigma} & \text { Desvio Padrão } \\ \boldsymbol{\sigma}^{\mathbf{2}} & \text { Variância } \\ \mathbf{\Omega} & \text { Ohm }\end{array}$




\section{SUMÁRIO}

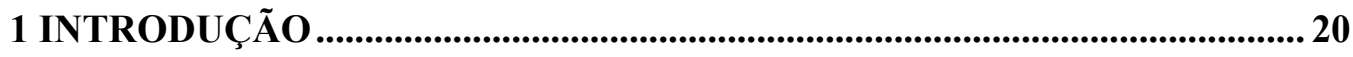

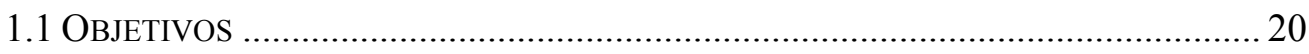

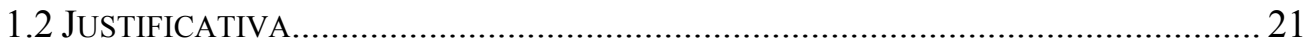

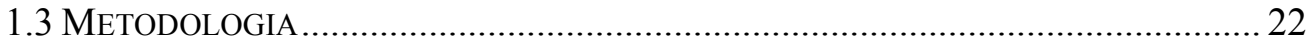

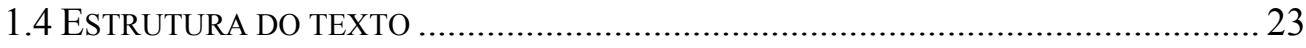

2 TRANSDUTORES INTELIGENTES E APLICAÇÕES EM ENGENHARIA

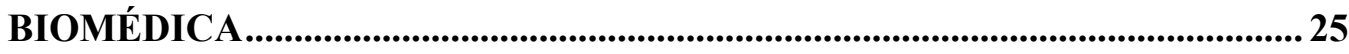

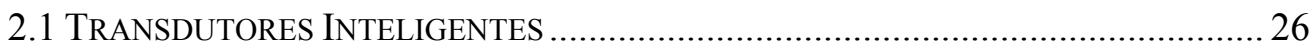

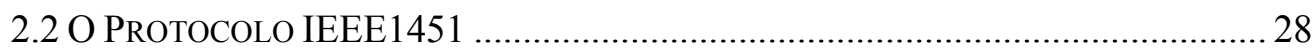

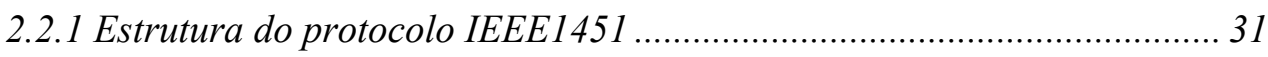

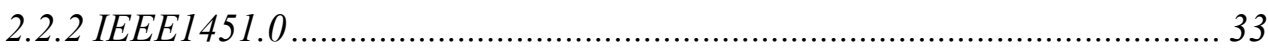

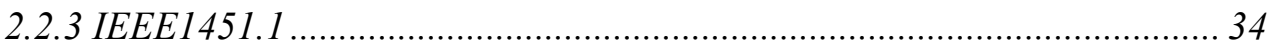

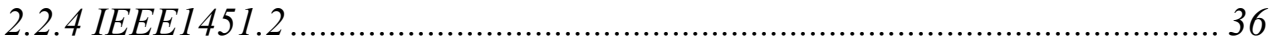

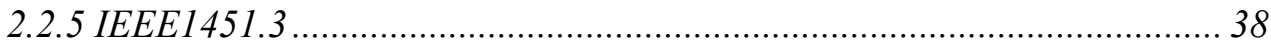

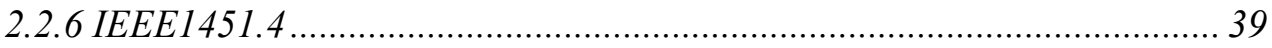

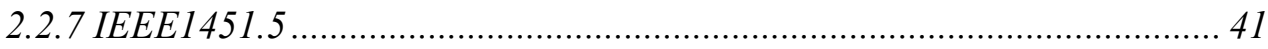

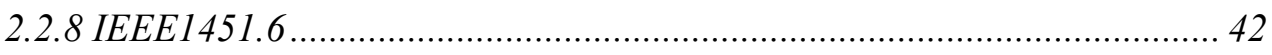

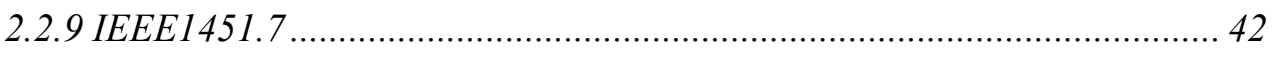

2.3 IMPLEMENTAÇÕES E FRAMEWORKS DO IEEE1451 ............................. 43

2.4 MÉTODOS DE ACESSO REMOTO - WEB SERVICES ................................. 45

2.4.1 Arquitetura de Web Services.................................................................... 46

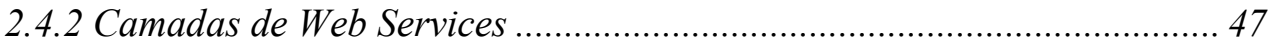

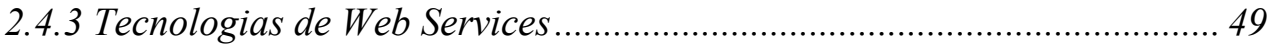

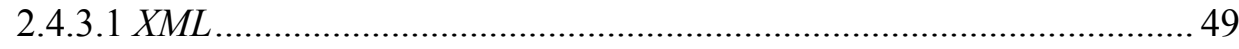

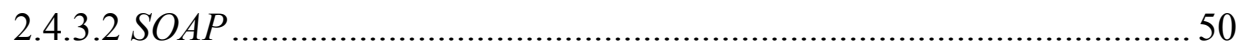

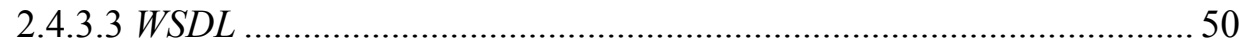

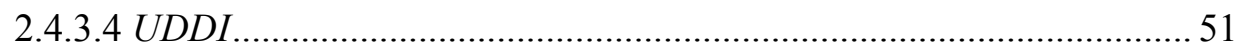


2.5.1 Eletrofisiologia do coração 53

2.5.2 Eletrocardiograma: medidas, monitorização e diagnóstico...................... 54

2.5.3 Derivações eletrocardiográficas.............................................................. 56

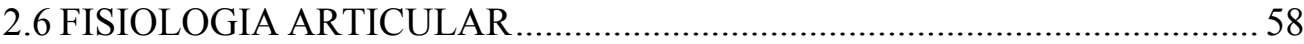

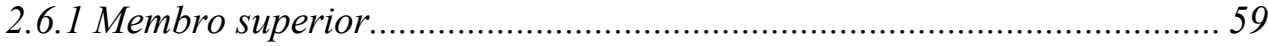

\section{DESENVOLVIMENTO DOS NÓS BIOMÉDICOS UTILIZANDO O}

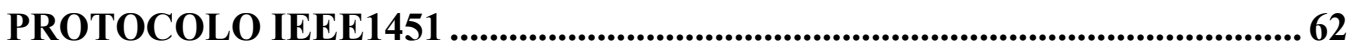

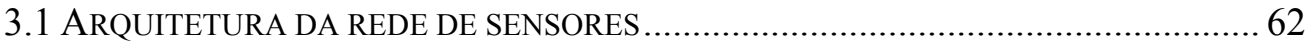

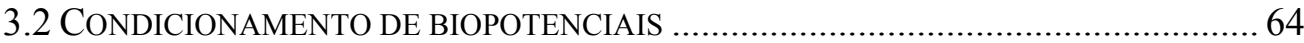

3.2.1 Arquitetura do circuito condicionador de biopotenciais ......................... 65

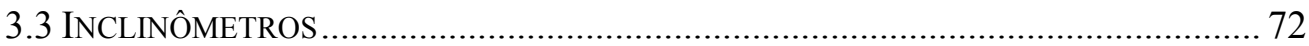

3.4 SISTEMAS EMBARCADOS MICROCONTROLADOS................................................. 75

3.4.1 Interface serial USB ............................................................................. 76

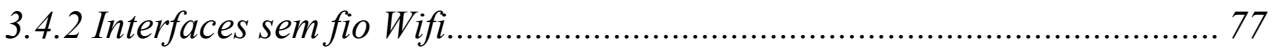

3.5 DESENVOLVIMENTO E IMPLEMENTAÇÃO Do IEEE1451.0 .................................. 78

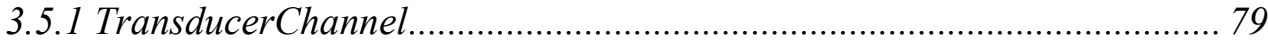

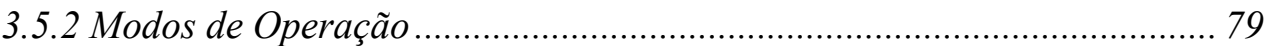

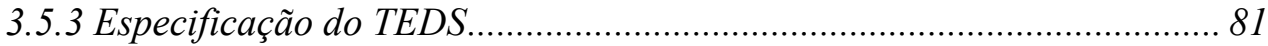

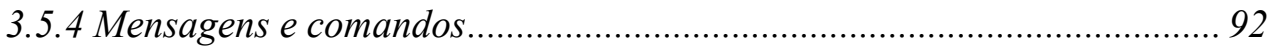

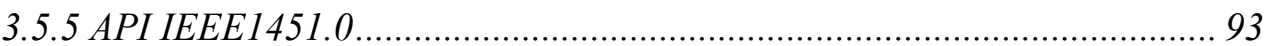

3.6 DESENVOLVIMENTO E IMPLEMENTAÇÃO DO IEEE1451.2 ….............................. 94

3.7 DESENVOLVIMENTO E IMPLEMENTAÇÃO Do IEEE1451.5 .................................. 98

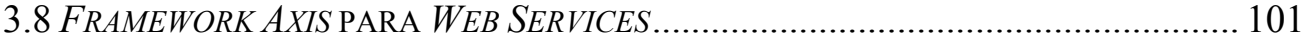

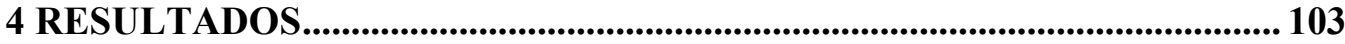

4.1 SIMULAÇÃO E ANÁLISE DO CIRCUITO DE CONDICIONAMENTO DE BIOPOTENCIAIS 103

4.1.1 Caracterização do circuito de condicionamento de biopotenciais........ 109

4.2 CARACTERIZAÇÃO DOS ACELERÔMETROS COMO INCLINÔMETROS ................... 112

4.2.1 Medida de inclinação com um acelerômetro ........................................... 112

4.2.2 Medida de ADM com dois acelerômetros............................................... 118 
4.3 Prototipagem dos SiSTEMAS EMBARCADOS MiCROCONTROLADOS 121

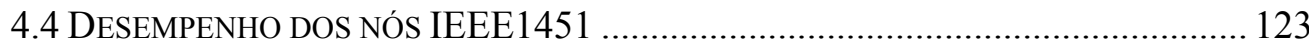

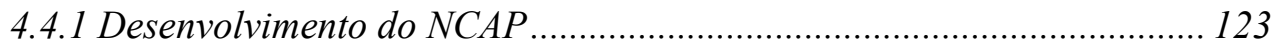

4.4.2 Desenvolvimento dos nós IEEE1451.2 …........................................ 125

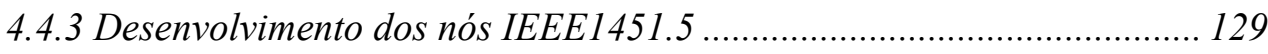

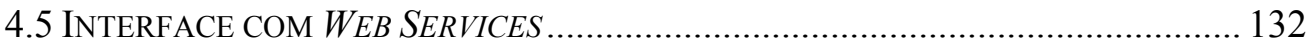

4.6 VALIDAÇÃO DO WTIM COM O FRAMEWORK NIST ......................................... 135

4.7 INTEGRAÇÃO DA REDE DE SENSORES PARA ENGENHARIA BIOMÉDICA ............. 138

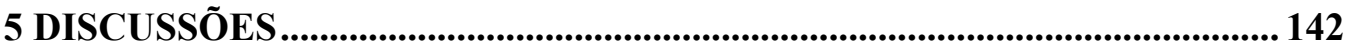

5.1 SENSORES BIOMÉDICOS E SISTEMAS MICROCONTROLADOS ....... 142

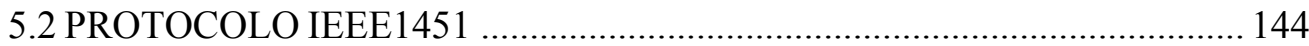

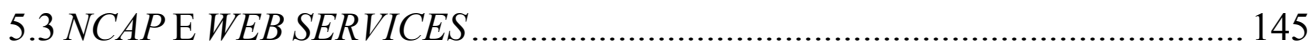

6 CONCLUSÕES E TRABALHOS FUTUROS .............................................. 147

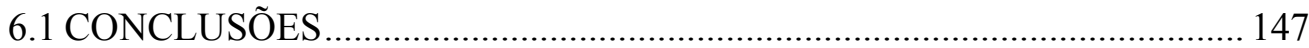

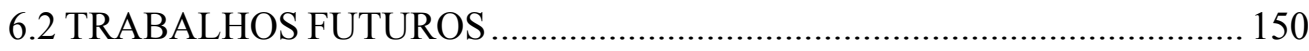

7 TRABALHOS APRESENTADOS .......................................................... 153

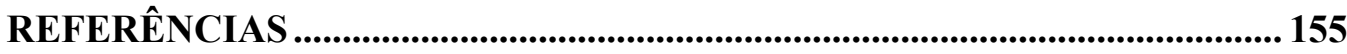

APÊNDICE A - SIMULAÇÃO DO CIRCUITO DE CONDICIONAMENTO DE BIOPOTENCIAIS ................................................................................................... 165

APÊNDICE B - SIMULAÇÃO DO CIRCUITO CONDICIONADOR COM INSTRUMENTOS VIRTUAIS............................................................... 166 APÊNDICE C - ESQUEMA DO CIRCUITO DE CONDICIONAMENTO DE BIOPOTENCIAIS - PARTE 1 ....................................................................... 167 APÊNDICE D - ESQUEMA DO CIRCUITO DE CONDICIONAMENTO DE BIOPOTENCIAIS - PARTE 2 ................................................................................. 168 APÊNDICE E - ESQUEMA DO CIRCUITO DE CONDICIONAMENTO DE

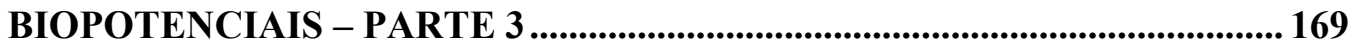
APÊNDICE F - CARACTERIZAÇÃO DO ACELERÔMETRO MX2125 EIXO X. 
APÊNDICE G - CARACTERIZAÇÃO DO ACELERÔMETRO MX2125 EIXO Y

APÊNDICE H - CARACTERIZAÇÃO DO ACELERÔMETRO MX2125 .... 172 APÊNDICE I - CARACTERIZAÇÃO DO MX2125 PARA MEDIDA DE AMPLITUDE DE MOVIMENTO 173

APÊNDICE J - ESQUEMA DE POLARIZAÇÃO DO MICROCONTROLADOR ATMEGA8535 174

APÊNDICE K - ESQUEMA DE POLARIZAÇÃO DO

MICROCONTROLADOR AT90USB162. 175

ANEXO A - MICROCONTROLADOR ATMEGA8535 176

ANEXO B - MÓDULO WIFI WIFLY GSX 802.11B/G 177

ANEXO C - MICROCONTROLADOR AT90USB162 ................................... 178

ANEXO D - ACELERÔMETRO PARALLAX MX2125.................................... 179

ANEXO E - META TEDS - IEEE1451.0 .............................................................. 180

ANEXO F - TRANSDUCERCHANNEL TEDS - IEEE1451.0 ....................... 181

ANEXO G - USER'S TRANSDUCER NAME TEDS -IEEE1451.0 ................. 182

ANEXO H - PHY TEDS - IEEE1451.2 _........................................................ 183

ANEXO I - PHY TEDS - IEEE1451.5 ......................................................... 184 


\section{INTRODUÇÃO}

\subsection{Objetivos}

O objetivo principal deste trabalho foi desenvolver uma instrumentação de aquisição de sinais associados ao protocolo de rede de sensores inteligentes IEEE1451 para aplicações em engenharia biomédica. Dentro desse contexto foram propostos o projeto e o desenvolvimento de sistemas embarcados microprocessados, que realizaram o monitoramento de sensores por uma rede de sensores Plug and Play com conexão via Internet através de uma arquitetura Web Services.

Ou seja, foram focos do trabalho: o desenvolvimento de sensores para tomada de medidas que auxiliem no diagnóstico e interface para engenharia biomédica, a exemplificar, sensores de amplitude do movimento no desenvolvimento de eletrogoniômetros e sensores de biopotenciais que condicionem sinais de eletrocardiogramas; o desenvolvimento de um sistema microcontrolado, capaz de realizar aquisições analógico-digitais, armazenamento dos dados e interface através de comunicações USB e Wifi $(802.11 \mathrm{~b} / \mathrm{g})$; a programação de softwares em linguagens ANSI C, $\mathrm{C}++$ e Java que padronizem os nós sensores de acordo com o protocolo IEEE1451, em específico, o IEEE1451.0, IEEE1451.2, IEEE1451.5; e a disponibilização através de Web Services das informações coletadas e padronizadas através dos sensores, favorecendo a utilização de recursos de instrumentação virtual e suas aplicações em um Laboratório Remoto.

Como objetivos secundários, o projeto também abriu possibilidades de aplicações, sem prejuízo da funcionalidade, em monitoramento ambiental com potencialidade de utilização de sensores de temperatura, luminosidade, radiação, $\mathrm{pH}$, umidade, concentração de dióxido de carbono e vapores de gases tóxicos em lugares distantes e inóspitos. Além disso, em sistemas de controle industriais para controle de variáveis em processos. 


\subsection{Justificativa}

A introdução de um novo protocolo seja de comunicação ou de padronização de linguagens, técnicas e procedimentos sempre se choca com interesses de fabricantes, empresas e usuários. A aceitação por parte desses pode ocorrer ou através de uma imposição política e estratégica ou por consenso de mérito e qualidade do protocolo. O IEEE1451 se situa nesse segundo grupo de protocolos. Desde sua idealização em 1993 até a atualidade, o protocolo nunca teve a pretensão de suprimir outros padrões já existentes e estabelecidos. Pelo contrário, o IEEE1451 priorizou o englobamento e encapsulamento dos outros protocolos, a permitir ao usuário uma interface de comunicação de transdutores inteligentes indiferente da rede de controle.

Essa característica, aliada a outras virtudes do IEEE1451, incentivaram fabricantes a repensarem acerca dos seus equipamentos e transdutores, bem como permitiu a academia realizar pesquisas e propor melhorias e novas funcionalidades. Entretanto cabe lembrar que o protocolo é recente, por isso ainda carece de aplicações e testes. A pesquisa acerca do protocolo vem rendendo boas discussões sobre algumas modificações em curso.

Em específico, trabalhos desenvolvidos acerca do protocolo IEEE1451 deixaram um legado de estudos e implementações, na sua maioria, acerca do padrão IEEE1451.2 (BATISTA, 2004) (ROSSI, 2005) (TANI, 2005) (VIEGAS, 2004) ou do IEEE1451.4 (SANTOS, 2005) (OLIVEIRA, 2005). Entretanto a partir de 2007 houve uma reviravolta na família de protocolos que constituem o IEEE1451. Nesse ano foi aprovado o protocolo IEEE1451.0, que além de possuir um índice remissivo, mudou a forma de abordagem dos outros protocolos, com exceção do IEEE1451.4. Esse novo rumo tomado pelo IEEE (Institute of Electrical and Electronics Engineers) em parceria com o NIST (National Institute of Standards and Technology) teve como objetivo tornar o protocolo mais acessível e operável. Dessa virada, todos os trabalhos até então apresentados tornaram-se obsoletos, o que, é claro, não desmereceu e nem desprestigiou a pesquisa realizada. 
A novidade apresentada nessa nova forma de organização do IEEE1451 requer, além de testes, implementações de fato. Nota-se que os atuais exemplos de aplicação, provenientes, na sua maioria, do grupo de pesquisa de Kang Lee ${ }^{1}$, têm como objetivo servir muito mais como prova de conceito do que como implementação de fato em engenharia, utilizando sensores ou atuadores em problemas reais.

Coube então, a partir da percepção que o protocolo IEEE1451 possui um grande potencial aliado com as atuais mudanças propostas pelo IEEE, retirar do "papel" esse protocolo e trazer para aplicações que utilizem sensoriamento como ponto fundamental de desenvolvimento. Optou-se por utilizar aplicações em engenharia biomédica como forma de demonstrar a utilização do IEEE1451 em aplicações não canônicas, posto que na literatura são observados, em larga medida, sensores de temperatura, pressão, entre outros.

\subsection{Metodologia}

As etapas constitutivas do projeto versaram, primeiramente, a revisão bibliográfica acerca dos temas fundantes: o protocolo IEEE1451, o desenvolvimento de sistemas embarcados microcontrolados e interfaces de comunicação; engenharia biomédica: eletrofisiologia do coração e fisiologia articular; e tecnologias Web Services.

A parte experimental do projeto ficou a cargo do desenvolvimento de sensores e sistemas de condicionamento utilizando microprocessadores. Bem como da codificação em linguagem de programação ANSI C, C $++^{2}$ e Java, dos protocolos publicados IEEE1451.0, IEEE1451.2 e IEEE1451.5. Os programas desenvolvidos foram utilizados tanto no sistema microcontrolado dedicado à aquisição de sinais como no sistema de monitoramento e disponibilização de dados que pode ser um computador pessoal ou um servidor.

\footnotetext{
${ }^{1}$ Líder do grupo de Desenvolvimento de Sensores e Aplicações no Laboratório de Engenharia de Manufatura do NIST.

${ }^{2} \mathrm{C}++$, cujo nome originalmente era de $\mathrm{C}$ com classes.
} 
Em relação ao sistema embarcado, são apresentados o desenvolvimento de uma eletrônica digital e analógica em placa de circuito impressa capaz de filtrar, amplificar e condicionar biopotenciais e informações provenientes de acelerômetros integrados.

O software de monitoramento programado em linguagem Java, tem o objetivo de disponibilizar os dados localmente através de uma interface gráfica e remotamente através de um Web Service implementado a partir do framework Apache Axis $2^{3}$ Java e independente de sistema operacional.

Os testes feitos com o protótipo funcional tiveram como objetivo a caracterização dos sensores e dos sistemas embarcados. Além disso, o trabalho também teve como foco certificar a característica de conexão e reconhecimento rápido (Plug and Play), sua funcionalidade como sistema de instrumentação para aquisição de dados e sua potencialidade de funcionar remotamente.

\subsection{Estrutura do texto}

Este primeiro capítulo faz uma introdução ao tema, ao problema e ao recorte dado para o projeto. No segundo capítulo é feita uma breve descrição da evolução de sistemas de transdutores. Esse histórico demonstra como os diferentes paradigmas de automação foram modificados a permitir novas funcionalidades e demandas. Tal evolução culmina, atualmente, com o conceito de transdutores inteligentes e dentro desse contexto são definidas as bases da família do protocolo IEEE1451.

Nesse mesmo capítulo são tratados os métodos de acesso remoto, em especial, o uso de Web Services. Essa tecnologia de computação distribuída ampliou a utilização de middlewares que permitem a mediação entre usuários remotos e sistemas de sensoriamento e controle.

Ao fim do capítulo, faz-se menção da literatura que serve como base para as duas aplicações de engenharia biomédica abordadas nesse trabalho. No subitem "Anatomia e fisiologia do coração" são apresentadas a origem e as formas do

\footnotetext{
${ }^{3}$ Disponível no site: ws.apache.org/axis/
} 
eletrocardiograma. Já "Fisiologia articular" apresenta os padrões para medição de amplitude de movimento de uma articulação e sua utilização em fisioterapia.

No terceiro capítulo, descreve-se a arquitetura do trabalho. Para tanto, são detalhados os materiais e métodos utilizados na construção da rede de sensores. Desde o desenvolvimento dos sensores, passando pela escolha dos microcontroladores e interfaces de comunicação, na especificação dos protocolos IEEE1451 e no detalhamento do framework de Web Services Axis2.

No quarto capítulo são apresentados os resultados da caracterização dos sensores e dos sistemas embarcados. Além disso, descreve-se o desenvolvimento do protocolo IEEE1451 e o desempenho dos nós IEEE1451.0, IEEE1451.2 e IEEE1451.5. São também apresentadas as interfaces Web Services que permitem o acesso remoto da informação coletada. Dando sequência ao capítulo é feita a validação do sistema sem fio através do framework desenvolvido por Song e Lee (2006) e, por último, a integração do sistema com intuito de demonstrar a funcionalidade da rede de sensores desenvolvida.

O quinto capítulo discute a aplicabilidade dos resultados obtidos com a padronização da comunicação entre sensores e redes de controle, a demonstrar os benefícios e limitações do protocolo IEEE1451. Dessa maneira conclui-se, com o sexto capítulo, a potencialidade do protocolo em questão. Por último, no capítulo sétimo, são sugeridas novas pesquisas a partir das experiências adquiridas no desenrolar desse trabalho. 


\section{TRANSDUTORES INTELIGENTES E}

\section{APLICAÇÕES EM ENGENHARIA BIOMÉDICA}

Transdutores estão por toda a parte (LEE, 2008). Esses dispositivos têm como base de funcionamento a conversão de energia de uma forma para outra. $\mathrm{O}$ termo transdutor refere-se a um grupo especializado de dispositivos. Esses podem ser sensores ou atuadores, sendo que sensores são transdutores que geram sinais elétricos proporcionais a parâmetros físicos, biológicos, ou químicos. E atuadores são transdutores que transformam sinais elétricos em ações físicas (SONG; LEE, 2008a) (NORTON, 1989) (JONES; CHIN, 1991).

Atualmente sensores e atuadores são considerados componentes fundamentais na engenharia, cujas aplicações versam o desenvolvimento técnico e industrial na monitoração e controle de processos, desde a obtenção do produto bruto até seu produto final. Graças aos avanços em microeletrônica a partir da década de 70, a tecnologia de sensores permitiu a evolução dos sistemas de automação, culminando no que se compreende hoje como sensores inteligentes (PAVEL, 2007).

Essa evolução dos sistemas de sensores e atuadores teve início com a utilização de painéis de controle fixos eletromecânicos, baseados em relês. Devido a dificuldades como cabeamento, capacidade de trabalho, espaço e manutenção, esses foram substituídos por sistemas digitais, mais flexíveis e escaláveis. A partir desses controladores digitais foi possível atingir maior confiabilidade e robustez, menor tamanho e custo, tornando esses sistemas um padrão na indústria com o nome de controladores lógicos programáveis (CLPs) (BRYAN; BRYAN, 1997 apud TANI, 2006).

Os CLPs flexibilizaram o sensoriamento e o controle, pois utilizavam uma programação simples, baseada em diagramas ladder, que já era utilizada nos sistemas tradicionais. Entretanto as aplicações aumentaram de tamanho e de complexidade. Dessa forma, a arquitetura centralizada dos CLPs se tornou onerosa em termos de cabeamento, instalação e manutenção, devido ao fato desses sistemas concentrarem todo o processamento e tratamento das entradas e saídas em um único ponto. A 
tornar proibitiva a ampliação do sensoriamento e controle em um ambiente industrial.

Uma solução encontrada para melhorar o controle e monitoração dos transdutores e permitir maior escalabilidade foi a utilização de sistemas distribuídos. Esses sistemas se caracterizaram pela multiplicidade de nós de processamento e pela comunicação direta entre si através de um ou mais canais de comunicação usando protocolo comum (SAMAD; MCLAUGHLIN; LU, 2007). Em outras palavras, ao invés de vários sensores e atuadores diretamente conectados a uma CLP, conectavase esses transdutores em barramentos, que por sua vez, estavam interligados aos controladores.

Essa arquitetura simplificou o cabeamento, a manutenção e a alteração dos nós do sistema (XIE; PU; MOORE, 1998 apud CESCHINI, 2008). Com ela os módulos de entrada e saída ficaram mais próximos do processo e das informações dos sensores e atuadores. Além disso, outro avanço a ser citado foi a transmissão das informações ao controlador central de forma digital. Essa transmissão foi realizada, primeiramente, através de um barramento de comunicação conhecido como fieldbus. Entre as vantagens dessa abordagem estavam a redução do cabeamento e a redução dos custos do sistema de controle (TANI, 2006).

\subsection{Transdutores Inteligentes}

A maior aproximação entre o sistema de controle e os processos também permitiu o aumento da capacidade dos próprios sensores e atuadores. Funcionalidades como: processamento digital de sinais e execução de algoritmos de decisão foram transferidos para o nível do sensor. Logo, os dados "brutos" que seriam transmitidos através do barramento foram suprimidos, o que permitiu um aumento do número de transdutores e diminuiu as necessidades de banda de transmissão da rede (GILSINN; LEE, 2001).

Ou seja, com o advento dos microprocessadores e a grande disponibilidade de recursos para o processamento de sistemas digitais, foi possível introduzir uma 
elevada capacidade de computação aos transdutores (CHONG; KUMAR, 2003). Essa capacidade de um dispositivo integrar um elemento sensor/atuador, uma unidade de processamento, uma interface de comunicação e um software associado para condicionamento de sinal, calibração e diagnóstico originou o conceito de Transdutor Inteligente, esquematizado na figura 2.1 (ELMENREICH; PITZEK, 2003) (SONG; LEE, 2008a).

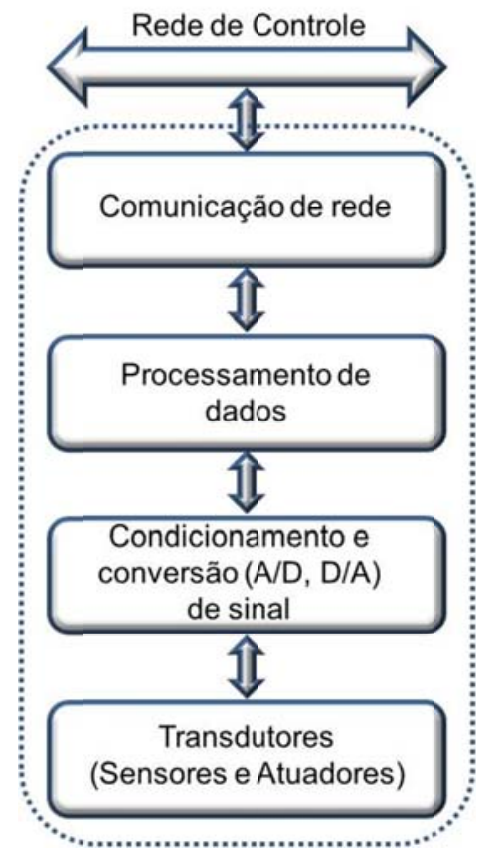

Figura 2.1 - Modelo de Transdutor Inteligente (SONG; LEE, 2008a).

Essa quebra de paradigma permitiu aos transdutores saírem de domínios exclusivos, como a automação industrial, e se dispersarem em outros ambientes, tais como: automação residencial, automação automotiva, automação agrícola, robótica, engenharia biomédica, entre outros. Essa ampliação de uso se apoiou também por áreas como a computação pervasiva ou ubíqua (WEISER, 1991). Nessas aplicações o uso de dispositivos embarcados ligados a sensores e redes de comunicação está totalmente integrado aos seres humanos. Para que isso ocorra são requisitos desses sistemas: escalabilidade, "inteligência", “invisibilidade" e interoperabilidade (SAHA; MUKHERJEE, 2003). 
Dentre as características apontadas acerca de Transdutores Inteligentes ainda há restrições e problemas de interoperabilidade ${ }^{4}$ nas tecnologias existentes. Em outras palavras, os dispositivos que englobam, atualmente, a ideia de Transdutores Inteligentes não possuem uma única maneira padronizada de comunicação. Nesse sentido o protocolo IEEE1451 foi idealizado como possível resposta para essa questão.

\subsection{O Protocolo IEEE1451}

Numerosas redes de controle foram desenvolvidas nas últimas duas décadas para diferentes tipos de aplicações. Esse fato fragmentou o mercado de automação, levando a uma diversidade de protocolos e padrões de comunicação para sistemas de transdutores. Esse excesso de técnicas e protocolos de interface diminuiu a interoperabilidade da instrumentação (SADOK; LISCANO, 2005). Algumas dessas padronizações encontradas no mercado são: Building Automation Control Network (BACNet), BITBUS, CEbus, ControlNet, Controller Area Network (CAN), Ethernet, Foundation FieldBus, Interbus, LonWorks, Local Interconnect Network (LIN-Bus), FlexRay, Modbus, Profibus e WorldFIP (LEE, 2001) (TANI, 2006).

A diversidade de redes de transdutores e a necessidade de acomodar sistemas que serão desenvolvidos no futuro têm guiado as pesquisas em direção de instrumentos que possam suportar redes heterogêneas e interoperabilidade entre sistemas (LEE, 2008). Em resposta às necessidades da indústria a uma interface padronizada de sensores, o IEEE propôs o desenvolvimento de uma interface padronizada para sensores e atuadores, conhecida como IEEE1451 (SONG; LEE, 2008a) (CORSO; BECARI, 2005) (BECARI et al., 2007). Essa solução para a padronização de sistemas de barramento distribuídos resultou no conjunto de normas da família IEEE1451.

O objetivo da família IEEE1451 (Standards for Smart Transducer Interface for Sensors and Actuators) é "definir um conjunto comum de interfaces para

\footnotetext{
${ }^{4}$ Compreende-se por interoperabilidade a habilidade de dois ou mais sistemas heterogêneos de trocar informações e usar esses dados de forma coerente.
} 
conectar transdutores inteligentes a redes, sistemas e instrumentos, através das tecnologias de comunicação emergentes" (LEE, 2001) (OLIVEIRA, 2005).

Em outras palavras, as normas IEEE1451 não propõem um novo protocolo de rede, em vez disso, elas definem um conjunto de interfaces normatizadas de hardware e software no sentido de separar o projeto dos transdutores da escolha das redes de comunicação (LEE, 2001). Sobre esse prisma o protocolo não impõe restrições aos aspectos construtivos dos transdutores, nem aos aspectos protocolares das redes. Ao separar as duas entidades, transdutores e redes, o modelo permite que o fabricante de transdutores se concentre no dispositivo de transdução, sem ter de se preocupar em adaptá-lo às muitas redes já existentes. Isso contribui para a melhoria da qualidade dos transdutores e para a redução dos seus custos. O objetivo final do modelo IEEE1451 é permitir o funcionamento imediato de qualquer transdutor em qualquer rede.

Outra característica importante da norma IEEE1451 é promover o desenvolvimento de dispositivos de conexão rápida (Plug and Play). Isso é possível através da padronização da documentação eletrônica do transdutor através do Transducer Electronic Data Sheet (TEDS). O TEDS contém as informações que permitem identificar e caracterizar os transdutores. Em uma palavra, é a documentação digital do sensor ou atuador. Ele deve ser configurado e agregado no próprio sensor/atuador em uma memória não volátil.

Nesse contexto a diferença no modelo dos Transdutores Inteligentes e dos Transdutores Inteligentes IEEE1451 é a adição do TEDS, a aparição do sistema em duas partes principais: o Network Capable Application Processor (NCAP) e o Transducer Interface Module (TIM). Esses conectados através de uma Transducer Independent Interface (TII). As figuras 2.1 e 2.2 estabelecem um comparativo entre os dois conceitos de inteligência apresentados.

Dentro do contexto do IEEE1451 um Transdutor Inteligente permite funcionalidades além das necessárias para gerar a correta representação de uma quantidade sensoriada ou controlada. Essas funcionalidades simplificam a integração de transdutores dentro de aplicações em redes de comunicações (SONG; LEE, 2008a). Transdutores IEEE1451 podem possuir características como: 
autoidentificação, autodescrição, capacidade de localização, "consciência" de tempo, processamento de dados, notificação de alertas, dados formatados e protocolos de comunicação.

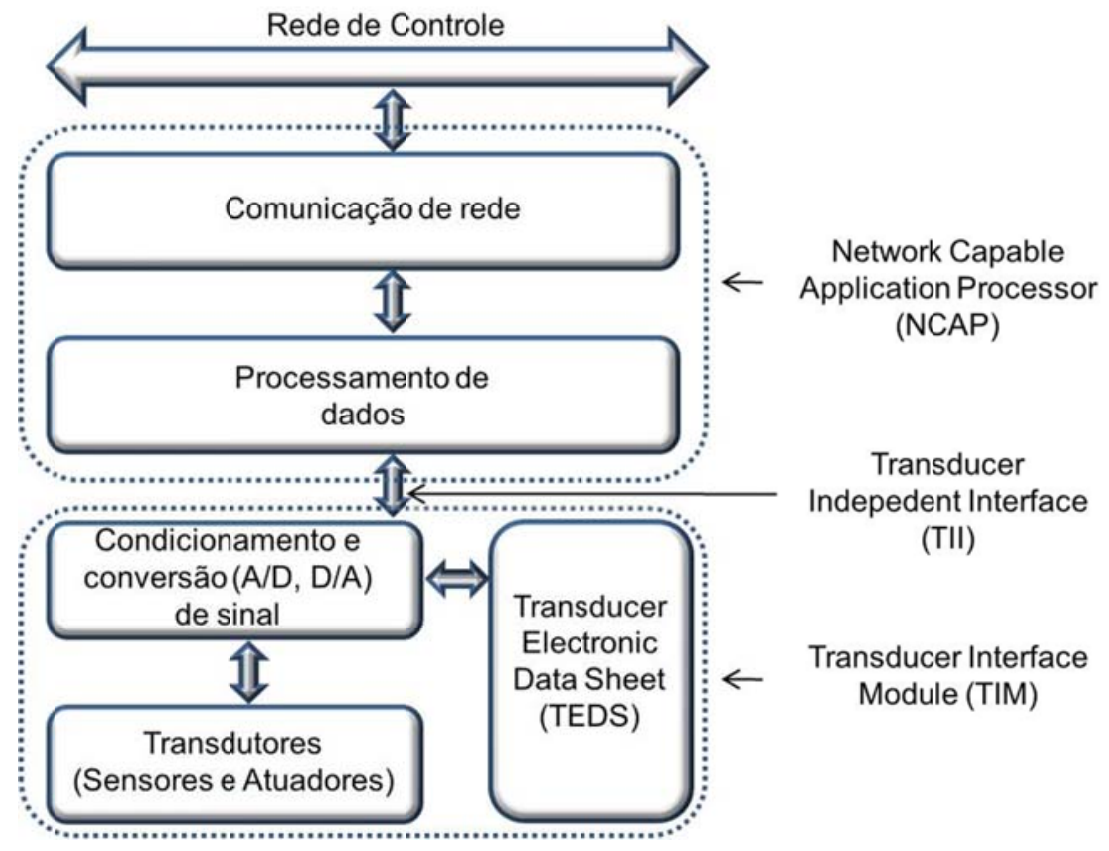

Figura 2.2 - Modelo de Transdutor Inteligente utilizando o protocolo IEEE1451 (SONG; LEE, 2008a).

Song e Lee (2008a) sintetizam os objetivos e especificidades da norma IEEE1451 através dos seguintes pontos:

- Desenvolver interfaces de transdutores independentes de rede e fabricante.

- Permitir que transdutores sejam substituídos ou movidos com o mínimo trabalho.

- Eliminar etapas de configuração manual sujeita a erros.

- Promover modelos genéricos para dados, controle, temporização, configuração e calibração de transdutores.

- Desenvolver uma documentação eletrônica no transdutor, que permita ao sensor se identificar em uma rede. Para isso se utiliza o TEDS, que pode 
documentar informações de localização, dia de recalibração, relatos de reparos e dados de manutenção.

- Possibilitar conexão Plug and Play, a permitir uma interface de comunicação para transdutores fisicamente separados (IEEE, 2004).

- Facilitar o suporte a múltiplas redes (IEEE, 2004).

Todas essas características apresentadas são especificadas e padronizadas nos protocolos que compõem a família IEEE1451. Essas normas estão apresentadas nos subcapítulos seguintes.

\subsubsection{Estrutura do protocolo IEEE1451}

Em 1993, ano do início do desenvolvimento das normas IEEE1451 (OLIVEIRA, 2005), o trabalho de criar uma interface comum para Transdutores Inteligentes foi separado em grupos. Cada grupo se encarregou de desenvolver e propor as normas específicas que, posteriormente, constituiriam a família do protocolo IEEE1451. Com o tempo esses grupos se estabeleceram e ao passo das publicações das normas, o protocolo se constituiu até o modelo atual apresentado pela figura 2.3.

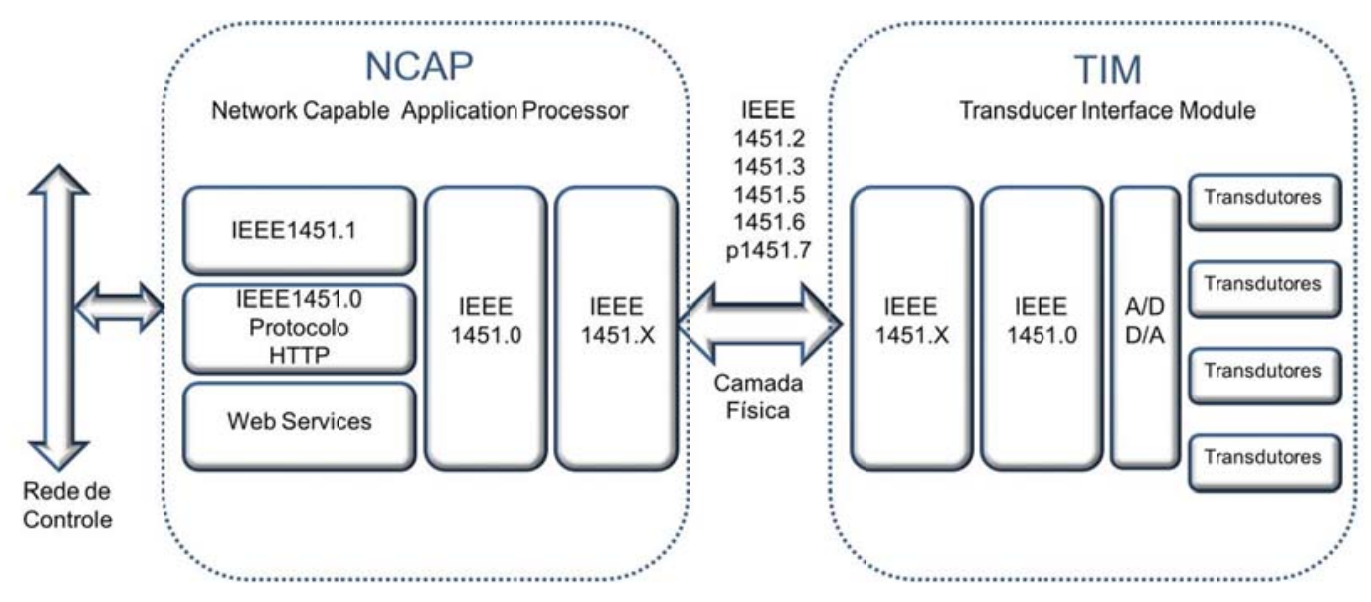

Figura 2.3 - Estrutura do Protocolo IEEE1451 (SONG; LEE, 2007). 
Desse modelo, faz-se uma breve descrição de cada norma, que serão especificadas posteriormente.

IEEE1451.0 - Essa norma define um conjunto APIs (Application Programming Interfaces) que caracterizam: comandos, operações e padrões de $T E D S$. Sua funcionalidade independe da camada física para comunicação entre transdutores e módulos de processamentos.

IEEE1451.1 - Define um modelo comum que descreve o comportamento de Transdutores Inteligentes, desde as medidas até a transmissão de dados.

IEEE1451.2 - Define uma conexão ponto a ponto entre transdutores e o $N C A P$. A norma original contemplava, apenas, a interface $T I I$, baseada na camada de comunicação SPI (Serial Peripheral Interface Bus) acrescida de linhas de controle, de fluxo e de temporização. Nas propostas atuais ${ }^{5}$, as interfaces foram ampliadas acrescentando as conexões: RS-232 (Recommended Standard 232) e RS-485 (Recommended Standard 485).

IEEE1451.3 - Define uma conexão em barramento entre transdutores e o $N C A P$. Na sua arquitetura é possível uma malha de nós transdutores compartilharem o mesmo par de fios.

IEEE1451.4 - Define uma interface mista para transdutores. Esses possuem tanto uma saída analógica como uma saída digital. A norma acresce aos transdutores uma memória descritiva TEDS.

IEEE1451.5 - Define uma interface de comunicação sem fio entre transdutores e o NCAP. A norma também contempla algumas definições particulares de integração do TEDS.

IEEE1451.6 - Define uma interface de comunicação através do barramento CANopen entre transdutores e o NCAP. Adota o perfil de dispositivos CANopen para dispositivos de medição e controladores em malha fechada.

IEEE1451.7 - Define uma interface de comunicação através de RFID (Radio-Frequency IDentification) entre sistemas de tags (transdutores ou de identificação) e redes de controle.

\footnotetext{
${ }^{5}$ Propostas que ainda estão em fase de pesquisa, mas não foram oficializadas pelo IEEE e pelo NIST.
} 


\subsubsection{IEEE1451.0}

A norma IEEE1451.0, Standard for a Smart Transducer Interface for Sensors and Actuators - Common Functions, Communication Protocols, and Transducer Electronic Data Sheet (TEDS) Format, cria uma lista de funções, protocolos e formatos comuns para facilitar a interoperabilidade entre todos os membros da família IEEE1451 que utilizam interfaces digitais (IEEE, 2007a). Em outras palavras, o objetivo do protocolo é atingir a interoperabilidade no nível de dados, quando múltiplos sensores com ou sem fio são conectados para formar o sistema (SONG; LEE, 2006).

Apesar de posterior às normas da família IEEE1451, sua intenção é compatibilizar os distintos meios físicos dos padrões já existentes. Além de possibilitar alto grau de compatibilidade entre os membros da família. Por ter sido proposta posteriormente, o padrão IEEE1451.0 ainda não esta totalmente compatível com os padrões IEEE1451.1-1999, IEEE1451.2-1997 e IEEE1451.3-2003. Já os padrões IEEE1451.5-2007, IEEE1451.6 foram baseados na utilização das funções, comandos e TEDS descritos no padrão em questão (IEEE, 2007a).

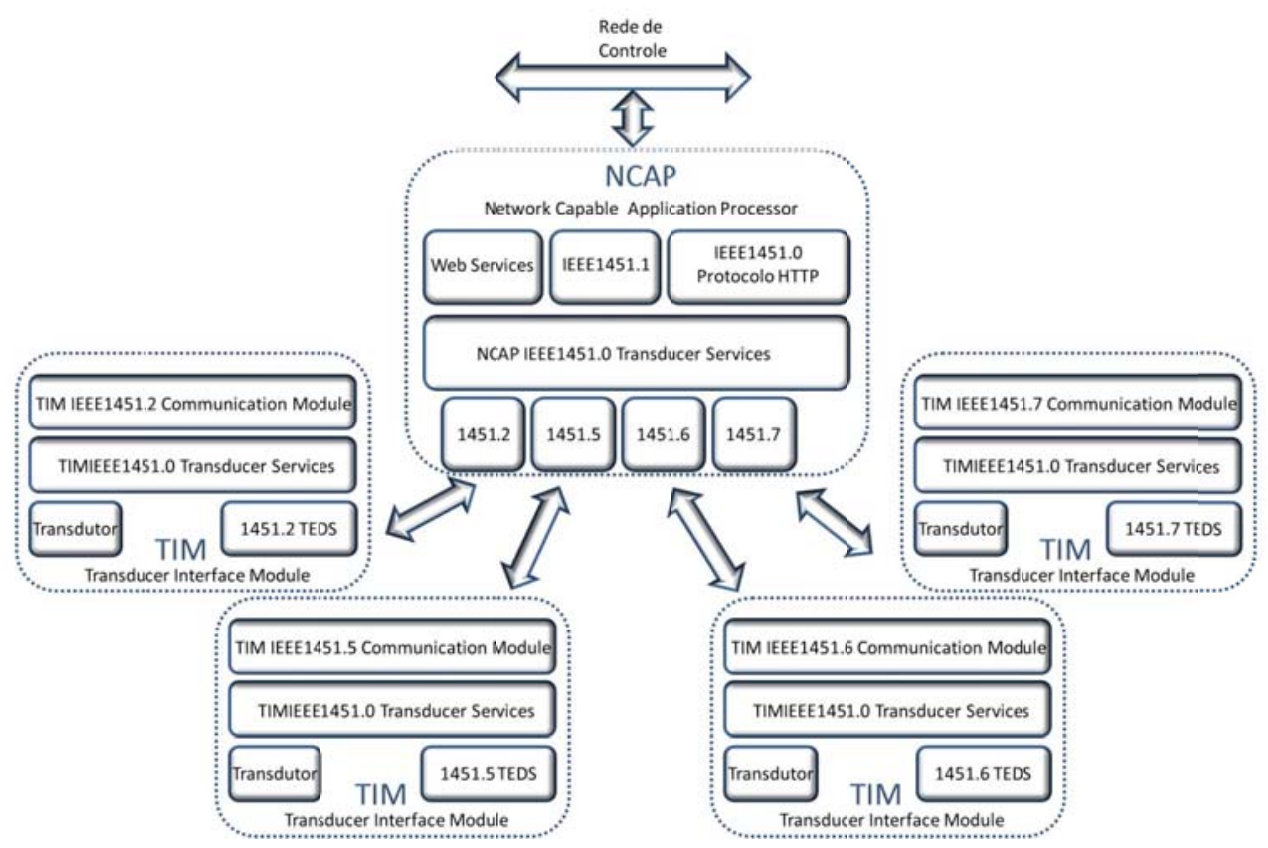

Figura 2.4 - Arquitetura da norma IEEE1451.0. 
O propósito do padrão é permitir aos fabricantes construir elementos interoperáveis dentro de sistemas de transdutores. Para realizar essa meta, a família de padrões IEEE1451.0 define duas categorias de dispositivos: o TIM, que corresponde ao módulo que engloba os transdutores, o conversor analógico-digital, a interface de comunicação e a memória TEDS. E o NCAP, que conecta os usuários em rede a esses módulos de transdutores remotos (IEEE, 2007a). A conexão entre TIMs e NCAPs é realizada através de uma interface física especificada por um membro da família IEEE1451. Tais características podem ser vistas na figura 2.4.

$\mathrm{Na}$ norma IEEE1451.0, um transdutor é considerado inteligente quando atende a três requisitos:

•É descrito por TEDS passíveis de serem lidos por computadores;

- O controle e os dados associados ao transdutor são digitais;

- O transdutor possui gatilhos (triggers), sinais de status e controle para monitorar e controlar o funcionamento dos transdutores (IEEE, 2007a).

A norma IEEE1451.0 também define as APIs disponíveis entre os diferentes blocos do modelo, a estrutura das mensagens trocadas entre NCAP e o TIM, o formato do TEDS e sua forma de acesso, os modos de operação e transferência que podem ser disponibilizados pelo TIM, o conjunto de comandos, a descrição das funções que devem ser realizados por um TIM e os serviços providos ao NCAP.

Essa norma também assegura uma descrição de formatação e comunicação. Contudo, como já mencionado anteriormente, a norma não descreve nada a respeito de transdutores e seus devidos processamentos digitais ou lógicos, posto que essas características são de responsabilidade do fabricante. Os únicos requisitos solicitados são de um hardware e software mínimos para acesso e controle da informação.

\subsubsection{IEEE1451.1}

A norma IEEE1451.1 - Standard for a Smart Transducer Interface for Sensors and Actuators - Network Capable Application Processor (NCAP) 
Information Model - tem como objetivo definir um modelo comum orientado a objeto de interface de software que gerencie a comunicação entre transdutores inteligentes e redes de acesso remoto de dados (IEEE, 2000) (OLIVEIRA, 2005).

A especificação abstrai detalhes referentes à configuração da arquitetura que deve ser construída, isto é, independe da tecnologia empregada. Esta especificação é composta por três definições fundamentais: o $N C A P$, que estabelece a modelagem propriamente dita do sensor inteligente conectado a uma rede; o modelo de dados, que define a formatação dos dados fornecidos pelo sensor; e o modelo de comunicação, que especifica as arquiteturas de comunicação com as redes permitidas (IEEE, 2000).

O NCAP engloba um conjunto de classes, métodos e atributos que podem ser agrupados em quatro grandes blocos (SVEDA, 2004):

- Classes - O bloco central do NCAP que providencia um ambiente de execução multitarefa independente, assegurando serviços básicos tais como o gerenciamento da memória, o tratamento de exceções e o gerenciamento de tarefas, o que inclui agendamento e a execução das tarefas concorrentes, bem como mecanismos de comunicação e sincronização entre elas.

- Funções - Os blocos de funções previstos na norma IEEE1451.1 constituem uma estrutura básica com as quais o desenvolvedor pode criar aplicações específicas. Estas estruturas não detalham métodos ou ferramentas para a aplicação a ser desenvolvida, mas orientam o seu desenvolvimento através da listagem dos parâmetros empregados no sistema para que a aplicação seja o mais transparente possível para o NCAP.

- Transdutor - O bloco de interface gerencia a comunicação entre o $N C A P$ e o transdutor. Este processo se inicia com a identificação do tipo do transdutor que está conectado ao sistema e, posteriormente, com a disponibilização dos métodos de leitura e escrita dos transdutores identificados. 
- Rede - O bloco de interface trata de todas as transações entre o $N C A P$ e a própria rede. A interação entre o $N C A P$ e a rede se baseia no conceito de chamada remota Remote Procedure Call (RPC). Esse método torna possível a um cliente remoto, de forma absolutamente transparente, instanciar uma classe do $N C A P$ e utilizar os seus atributos, métodos e eventos como se o objeto residisse no próprio cliente, adaptando desta forma, o conceito de serviço remoto para os transdutores.

Essa norma foi aprovada em 1999, entretanto foi substituída pela IEEE1451.0 em 2007, não fazendo mais parte das normas ativas. Alguns autores ainda fazem referência à norma pelos trabalhos que foram realizados em termos de pesquisa e aplicação.

\subsubsection{IEEE1451.2}

A norma IEEE1451.2 - Smart Transducer Interface for Sensors and Actuators - Transducer to Microprocessor Communication Protocols and Transducer Electronic Data Sheet (TEDS) Formats - define uma arquitetura de interface para a comunicação digital entre um módulo de transdução inteligente, Smart Transducers Interface Module (STIM), e um processador de rede NCAP, como apresentado na figura 2.5 (IEEE, 1998). A interface TII especifica as características elétricas e físicas da comunicação e sincronização.

Nos principais blocos do módulo STIM estão conectados todos os sensores e atuadores dos sistemas digitais e analógicos (OLIVEIRA, 2005). O STIM também é responsável pela aquisição, condicionamento e digitalização do sinal proveniente de sensores analógicos ligados diretamente a ele. O módulo STIM deve possuir um canal analógico de entrada ligado a um conversor analógico-digital, e/ou um canal analógico de saída que pode ser ligado a um conversor digital-analógico. Pode-se verificar na figura 2.5 que o bloco denominado TEDS é constituído por uma memória não volátil, no qual ficam armazenadas características do sensor e do atuador que estão conectados no módulo STIM. O IEEE1451.2 não prevê nenhuma restrição aos sensores e atuadores ligados ao STIM (SANTOS, 2006). 


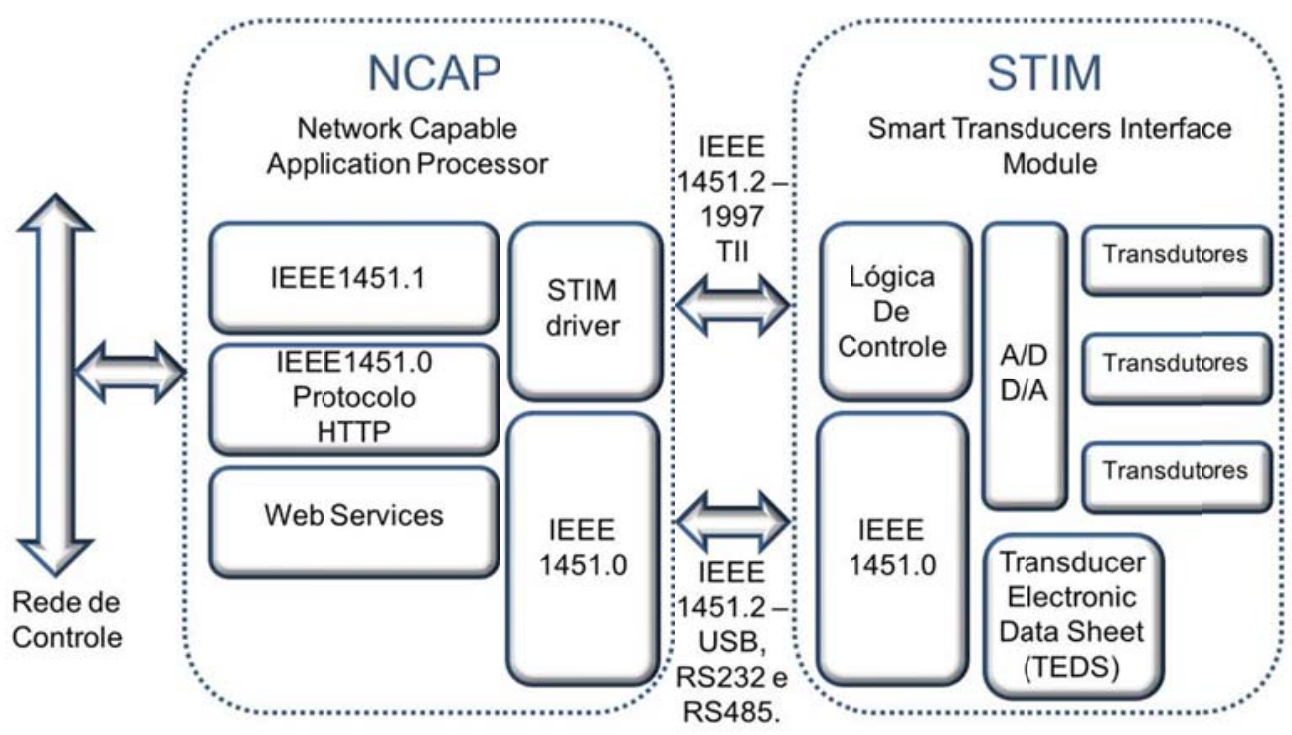

Figura 2.5 - Arquitetura da norma IEEE1451.2.

Na proposta de 1997 do protocolo IEEE1451.2 foram previstas oito estruturas de TEDS, que implementadas em memória não volátil, deveriam disponibilizar acesso à leitura e à escrita das informações contidas no transdutor. Entre as estruturas obrigatórias estavam: Meta TEDS, que representa as características gerais do STIM tais como identificador único, limites de tempos, números de canais e velocidade máxima de comunicação com o $N C A P$; e o TEDS de Canal, que possui propriedades particulares como o tipo de canal, unidades físicas, valores de máximo e mínimo, restrição temporal e incerteza. As estruturas opcionais podem ser resumidas por: $T E D S$ de Calibração, Meta TEDS de Identificação, TEDS de Identificação de Canal, $T E D S$ de Identificação de Calibração, TEDS do Usuário e TEDS para Extensão (IEEE, 1998) (VIEGAS, 200a).

Após a publicação da norma IEEE1451.0 foram propostas algumas modificações na IEEE1451.2. Entre elas a revisão dos padrões de TEDS, além da inclusão de outras interfaces de comunicação serial, como a $R S-232$, a $S P I$, a Universal Asynchronous Receiver/Transmitter (UART) e a USB como alternativa para a comunicação TII (SONG; LEE, 2008b) (RAMOS, 2007). 


\subsubsection{IEEE1451.3}

A norma IEEE1451.3 - Standard for a Smart Transducer Interface for Sensors and Actuators - Digital Communication and Transducer Electronic Data Sheet (TEDS) Formats for Distributed Multidrop Systems - define uma interface para sistemas de transdutores inteligentes distribuídos. Isso é realizado por meio de estruturas de memórias TEDS que permitem a autoidentificação dos transdutores, identificação e sincronização de canais, além de funções de escrita e de leitura do transdutor (VIEGAS, 2003). Tal como a norma IEEE1451.2, detalhes da implementação do sensor/atuador não são abordados pela norma (OLIVEIRA, 2005).

A estrutura do IEEE1451.3 associa um barramento comum ao processador de rede $N C A P$ e vários módulos de transdução inteligente, nomeados Transducer Bus Interface Module (TBIM). Cada TBIM pode ser associado ao barramento, que controlado por um Transducer Bus Controller (TBC) conectado ao NCAP, pode enviar e receber informações dos transdutores inteligentes (IEEE, 2004b).

O módulo TBIM é composto por um condicionador de sinais, um conversor analógico-digital e/ou digital-analógico e um barramento de interface que pode suportar até cinco canais de comunicação multiplexados em frequência sobre a linha de transmissão, permitindo que a alimentação dos módulos e a comunicação estejam presentes na mesma linha. Dos cinco canais de comunicação, apenas os canais de rede e de controle são obrigatórios.

A comunicação do TBIM é implementada utilizando o padrão HomePNA. Nesse padrão os sinais de comunicação, sincronização e de alimentação estão multiplexados na frequência, sendo que a alimentação é transmitida como sinal contínuo, o sinal de sincronização a $2 \mathrm{MHz}$ e as linhas de comunicação entre 4,75MHz e 9,25MHz (IEEE, 2004b). 


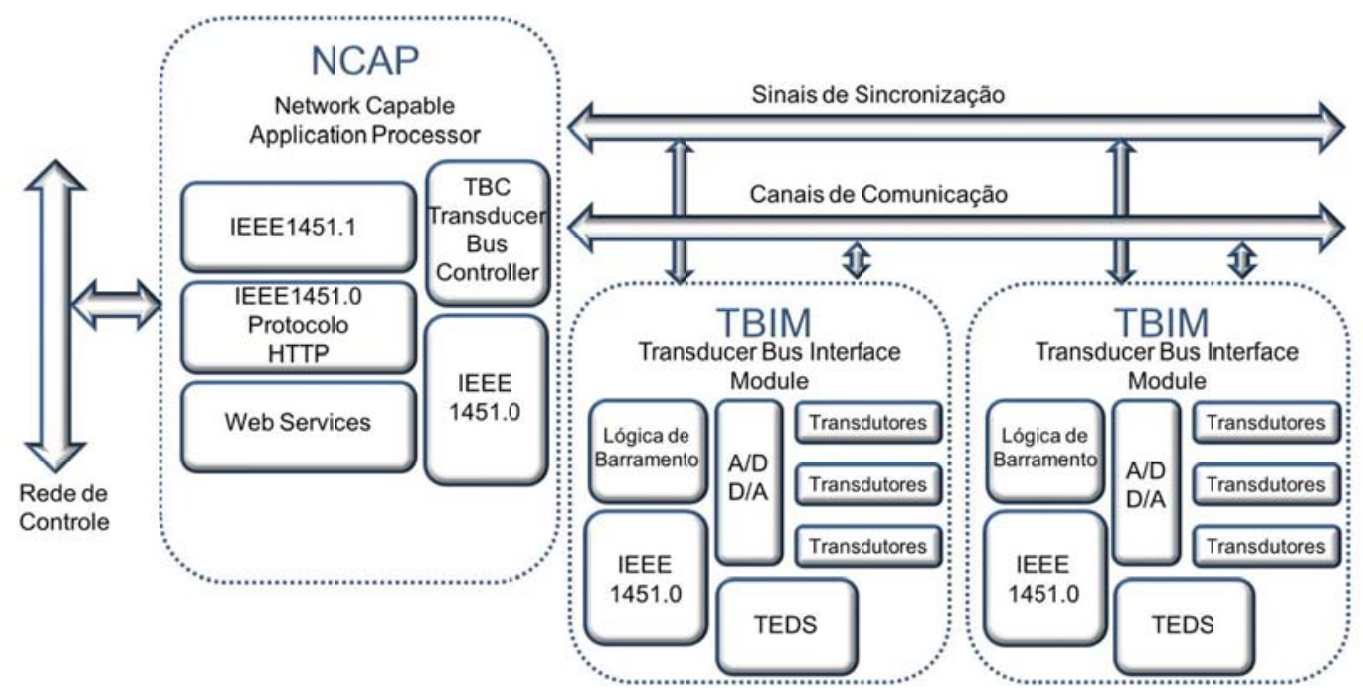

Figura 2.6 - Arquitetura da norma IEEE1451.3.

As estruturas de memória TEDS propostas pela norma IEEE1451.3 são uma variante das estruturas apresentadas pela norma IEEE1451.2. Essas estruturas e a especificação do TBIM podem ser analisadas na figura 2.6.

\subsubsection{IEEE1451.4}

A norma IEEE1451.4 - Standard for a Smart Transducer Interface for Sensors and Actuators - Mixed-Mode Communication Protocols and Transducer Electronic Data Sheet (TEDS) Formats - define uma interface para transdutores analógicos. De modo a compatibilizar os diversos tipos de transdutores existentes no mercado, sejam analógicos ou digitais (IEEE, 2004a) (SANTOS, 2006).

Para transformar um simples transdutor analógico em um transdutor Plug and Play e facilitar a integração com redes digitais são acrescidos a esses transdutores uma memória descritiva TEDS, que possibilita ao transdutor realizar sua autoidentificação e autocalibração. Além disso, é adicionado um módulo chamado Mixed Mode Interface (MMI), que possui uma interface de transmissão de dados mista, analógica e digital, pelos mesmos fios, apresentado na figura 2.7. 


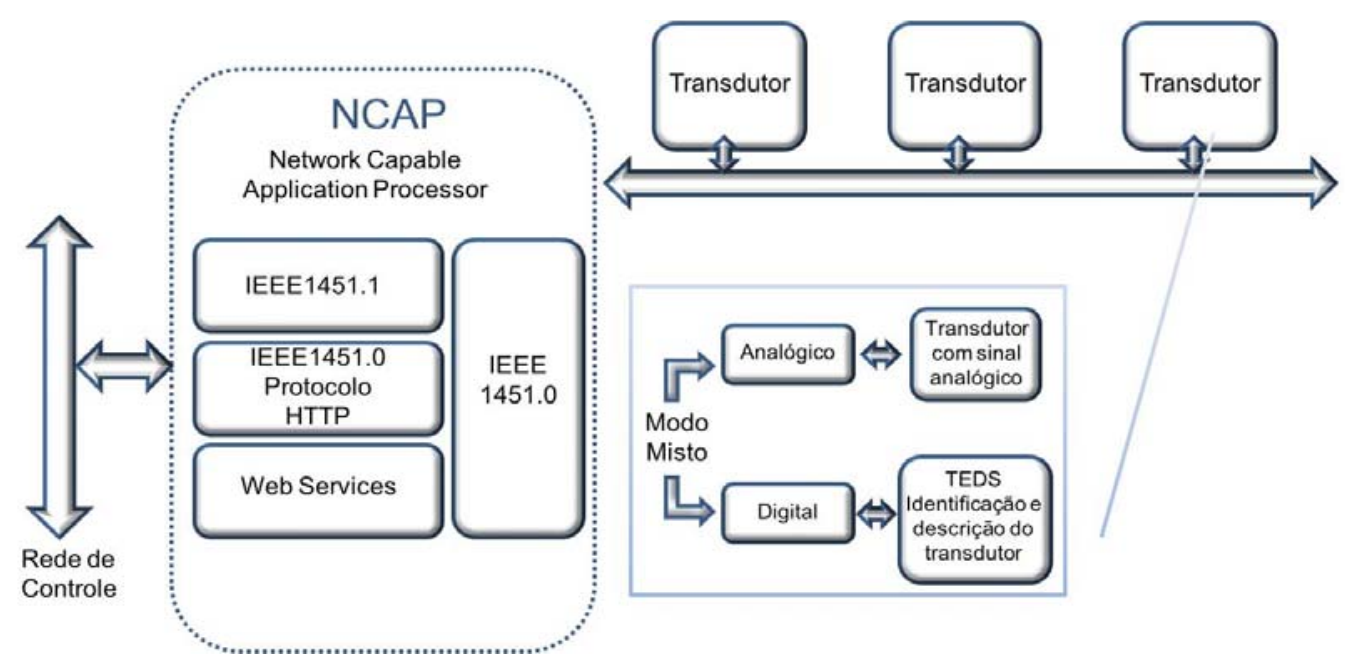

Figura 2.7 - Estrutura da norma IEEE1451.4.

A sobreposição da interface analógica com a digital pode ser realizada através de multiplexação no tempo, sendo essa chamada de classe 1 ou single wire. Por outro lado, se a interface digital for adicionada por meio de linhas próprias, em paralelo com a interface analógica, essa interface é denominada de classe 2, ou multi-wire (VIEGAS, 2003) (TANI, 2006).

As estruturas de memória TEDS propostas pela norma IEEE1451.4 são um subconjunto das estruturas contempladas na norma IEEE1451.2. Estão previstos campos para identificação do fabricante, modelo, número de série, entre outros (VIEGAS, 2003) (OLIVEIRA, 2005) (TANI, 2006). A maioria do TEDS também descreve características importantes dos transdutores, tais como: fundo de escala, sensibilidade, coeficientes de calibração e de interfaces elétricas.

A norma IEEE1451.4, desde sua definição em 2004, é a mais popular das propostas dentro da família IEEE1451, visto que esse conceito de sensores com autoidentificação já foi utilizado por várias empresas como National Instruments (NATIONAL, 2011) e a Honeywell Sensotec em sensores comerciais. 


\subsubsection{IEEE1451.5}

Atualmente as redes de sensores sem fio têm sido foco de desenvolvimento de várias áreas da pesquisa entre elas: militar, industrial, ambiental, logística e engenharia civil. Essa diversidade de aplicações fez com que o mercado de transdutores crescesse e tornasse diverso e heterogêneo (GILSINN; LEE, 2001). Cada fabricante utiliza tecnologias e padrões de comunicação de acordo com seus interesses ou facilidades. Essa falta de padronização dificulta a implementação de rede de transdutores sem fios nas mais diversas aplicações.

Para reduzir os riscos para usuários e empresas foi proposta a norma IEEE1451.5 - Standard for a Smart Transducer Interface for Sensors and Actuators - Wireless Communication Protocols and Transducer Electronic Data Sheet (TEDS) Formats. Essa norma estabelece um padrão de acesso a transdutores por uma interface de comunicação sem fio (IEEE, 2007b). Também estabelece métodos e formatos de dados baseado em TEDS para o acesso de dados dos transdutores remotamente localizados.

Dentre os protocolos de comunicação aprovados para a norma IEEE1451.5, foram adotados as tecnologias IEEE802.11 (WiFi), IEEE802.15.1 (Bluetooth), IEEE802.15.4 e ZigBee (IEEE, 2007) (CORSO; BECARI, 2006). Também foram reservadas possibilidades de incorporação de outros protocolos de comunicação, como é o caso do IEEE802.15.3 Ultra-Wide Band (UWB), IEEE802.16 (WiMax), entre outros.

A principal característica do protocolo é a introdução do conceito de Wireless Transducer Interface Module (WTIM). Esses módulos microprocessados que possuem uma interface sem fio acoplada com condicionadores de sinais, conversores analógico-digitais e/ou digital-analógicos e transdutores.

Por ser uma norma proposta recentemente, já há conformidade com a norma IEEE1451.0, com isso é possível constatar a mesma padronização dos dados armazenados no TEDS. A norma IEEE1451.5 também possui um NCAP, que permite uma interface transparente entre os transdutores e a rede de controle. Através dele é 
possível integrar os transdutores sem fio às redes de controle já existentes (TANI, 2006).

Dentre suas especificidades o IEEE1451.5 define o PHY TEDS, descrevendo a interface física de comunicação e suas propriedades. Ou seja, qual o tipo de sistema de rádio frequência realiza a comunicação entre o $W T I M$ e o módulo de rede $N C A P$, sua máxima banda de transmissão, entre outras características de transmissão.

\subsubsection{IEEE1451.6}

A norma IEEE1451.6 - Standard for a Smart Transducer Interface for Sensors and Actuators - A high-speed CANopen based Transducer Network Interface for Intrinsically Safe and non-Intrinsically Safe application - tem como objetivo estabelecer uma interface de comunicação entre o $N C A P$ e o módulo transdutor através de um barramento CANopen. O padrão define o uso de TEDS baseado no protocolo IEEE1451.0. Esses são mapeados no dicionário de entrada CANopen assim como as mensagens de comunicação, os parâmetros de configuração e a informação de diagnóstico (IEEE, 2010b).

A escolha do barramento CANopen tem como vantagens uma comunicação segura, a disponibilidade de dispositivos comerciais já existentes e a demanda do mercado estabelecido (TANI, 2006). Além disso, o padrão IEEE1451.6 tem como principais aplicações transdutores utilizados em controladores de malha fechada para operação em ambientes de rede com múltiplos níveis de controle.

\subsubsection{IEEE1451.7}

Não há atualmente nenhum padrão de interface aberta entre transdutores e sistemas de Identificação por Rádio Frequência (RFID). Cada fabricante desenvolve sua própria interface, sem nenhuma padronização.

Para reverter essa situação o IEEE vem desenvolvendo o protocolo IEEE1451.7 - Standard for a Smart Transducer Interface for Sensors and Actuators - 
Transducers to Radio Frequency Identification (RFID) Systems Communication Protocols and Transducer Electronic Data Sheet Formats, que define métodos de interface de comunicação e formatação de dados que facilitam a comunicação entre sistemas de identificação e tags inteligentes (aqueles que possuem sensores integrados) com seus controladores de rede (IEEE, 2009). Até o momento essa norma ainda não foi concluída.

\subsection{IMPLEMENTAÇÕES E FRAMEWORKS DO IEEE1451}

Retomando o tema acerca do protocolo IEEE1451, faz-se necessário citar alguns trabalhos e referências desenvolvidas nos últimos anos. Durante a história do padrão IEEE1451 houve mudanças de rumo em relação a algumas de suas normas. Muito das pesquisas iniciais foram destinadas a implementações e discussões acerca dos padrões IEEE1451.1 e IEEE1451.2. Tais normas tiveram seus grupos de trabalho selecionados em Setembro de 1994.

A proposta inicial de criar uma interface comum de comunicação para transdutores inteligentes originou as normas: IEEE1451.2-1997, que englobava uma interface de comunicação de 10 fios $(T I I)$ e a utilização de memórias descritivas TEDS; e a norma IEEE1451.1-1999, que possuía um modelo de programação orientado a objeto para representar um transdutor inteligente genérico.

Em 2002, o grupo de trabalho responsável pelo desenvolvimento da IEEE1451.2 se reuniu para dar início ao processo de reavaliação da norma. Considerando as necessidades da indústria e a opinião do mercado, foram sugeridas algumas alterações para essa norma, sendo a mais importante a simplificação da interface TII e a alteração de alguns campos do TEDS. Soluções mais simples e menos custosas foram propostas, como interfaces seriais $R S-232, R S-485$, ou USB.

Em relação à norma IEEE1451.1, a partir de 2007 ela foi substituída pela norma IEEE1451.0. Essa modificação teve o intuito de padronizar o TEDS e as interfaces internas da família IEEE1451 (TANI, 2006). Contudo apesar de 
substituída, alguns trabalhos ainda citam a norma IEEE1451.1 como uma das interfaces de comunicação para acesso a transdutores (SONG; LEE, 2008b) (YONGPING; YUN; ZHIJIA, 2007).

Uma implementação do IEEE1451.1 anterior a norma IEEE1451.0 é a Java Distributed Data Acquisition and Control. A JDDAC compreende um conjunto de APIs open source que permite a conexão de aplicações em Java com sistemas de instrumentação que seguem as normas IEEE1451, em específico as normas IEEE1451.1, já citada, e a IEEE1451.2.

O $J D D A C$ define métodos para descrever o dispositivo final do sistema de instrumentação, indicando como eles e os dados gerados podem ser tratados por uma aplicação Java. Essas abstrações reduzem o tempo de desenvolvimento, pois evitam a necessidade de se desenvolver um sistema a partir de API's de baixo nível, como por exemplo, as que tratam da comunicação serial. Entre as API's estão: Java Transducer API, Java Measurement Calculus API, Java Measurement Dataflow API e Java Precision Clock Synchronization API (OLIVERIA, 2005).

Outros frameworks, aplicações semicompletas e reutilizáveis, anteriores a IEEE1451.0 foram desenvolvidos, em sua maioria no NIST, entretanto se mostraram mais como provas de conceito e de software, pois apenas as características básicas das normas eram implementadas para demonstração das capacidades que permitem a integração e interoperabilidade entre diferentes redes. Entre alguns exemplos estão os módulos projetados por: Song e Lee (2004) e Viegas, Pereira e Silva (2005) (2007).

Implementações que utilizam a norma IEEE1451.0 são ainda em pequeno número. Wobschall (2008a) menciona aplicações da norma em projetos de monitoração de sensores de gases (WOBSCHALL, 2006), monitoração de ventilação em ambientes condicionados (WOBSCHALL, 2001) e aplicações com interface Wifi utilizando integração das normas IEEE1451.0 e IEEE1451.5 (SONG; LEE, 2007). Tal fato, demonstra que poucas pesquisas foram realizadas na aplicação do IEEE1451 a problemas reais e aplicações específicas. 


\subsection{MÉTODOS DE ACESSO REMOTO - WEB SERVICES}

Como mencionado em Alonso (2004) o termo Web Services é utilizado com muita frequência, entretanto nem sempre com o mesmo significado. A priori, pode-se definir Web Services como uma tecnologia de sistemas distribuídos com arquitetura orientada a serviços Service-Oriented Architecture (SOA) (OLIVEIRA, 2005). Uma segunda definição proposta por Kreger (2001) define Web Services como uma interface que descreve uma coleção de operações que são acessíveis pela rede, através de mensagens $X M L$ padronizadas.

Um Web Service, na prática, é uma maneira de padronizar e integrar aplicações $W e b$ através de novos conceitos de interoperabilidade como a linguagem Extensible Markup Language (XML), o protocolo Simple Object Access Protocol $(S O A P)$, o descritor Web Services Description Language (WSDL) e o diretório Universal Description, Discovery and Integration (UDDI). Esses sistemas surgiram como uma evolução dos modelos de computação distribuída, dentre os quais são elencados o Remote Method Invocation (RMI), o Distributed Component Object Model (DCOM) e o Common Object Request Broker Architecture (CORBA). Essas tecnologias tiveram sucesso na integração de softwares em ambientes de redes locais e homogêneas (GOMES, 2009). A evolução dessas tecnologias é contemplada pelos Web Services, capazes de trabalhar em ambientes heterogêneos.

A tecnologia Web Services permite que uma aplicação invoque outra para efetuar desde tarefas simples até complexas, mesmo que as duas aplicações estejam em sistemas diferentes e escritas em linguagens diferentes. Entre outras características dessa tecnologia estão: disponibilidade de serviços, descrição de identificação, localização de serviços, interoperabilidade entre sistemas operacionais e linguagens de programação, além da padronização gerenciada pela World Wide Web Consortium ( $W 3 C$ ), o que reduz a heterogeneidade (ARRUDA JR., 2003) (ALONSO et al., 2004). 


\subsubsection{Arquitetura de Web Services}

Uma arquitetura de Web Services é baseada na interação de um Provedor de Serviços, um Consumidor de Serviços e um Registro de Serviços. Esses elementos estabelecem entre si operações de publicação, pesquisa e ligação. Tais blocos de um Web Service são descritos abaixo e seu o funcionamento é detalhado na figura 2.8.

- Provedor de Serviços (Service Provider) - É o elemento que cria o Web Service, além de descrever, registrar e publicar os serviços para o cliente.

- Consumidor de Serviços (Service Requester) - Qualquer aplicação que utilize um Web Service criado por um Provedor de Serviços é considerada um Consumidor de Serviços, numa palavra, um cliente. Isso é realizado a partir da descrição disponibilizada, o que possibilita ao consumidor obter o mecanismo para ligação com o Web Service.

- Registro de Serviços (Service Registry) - É um repositório no qual são registradas e publicas as informações dos serviços.

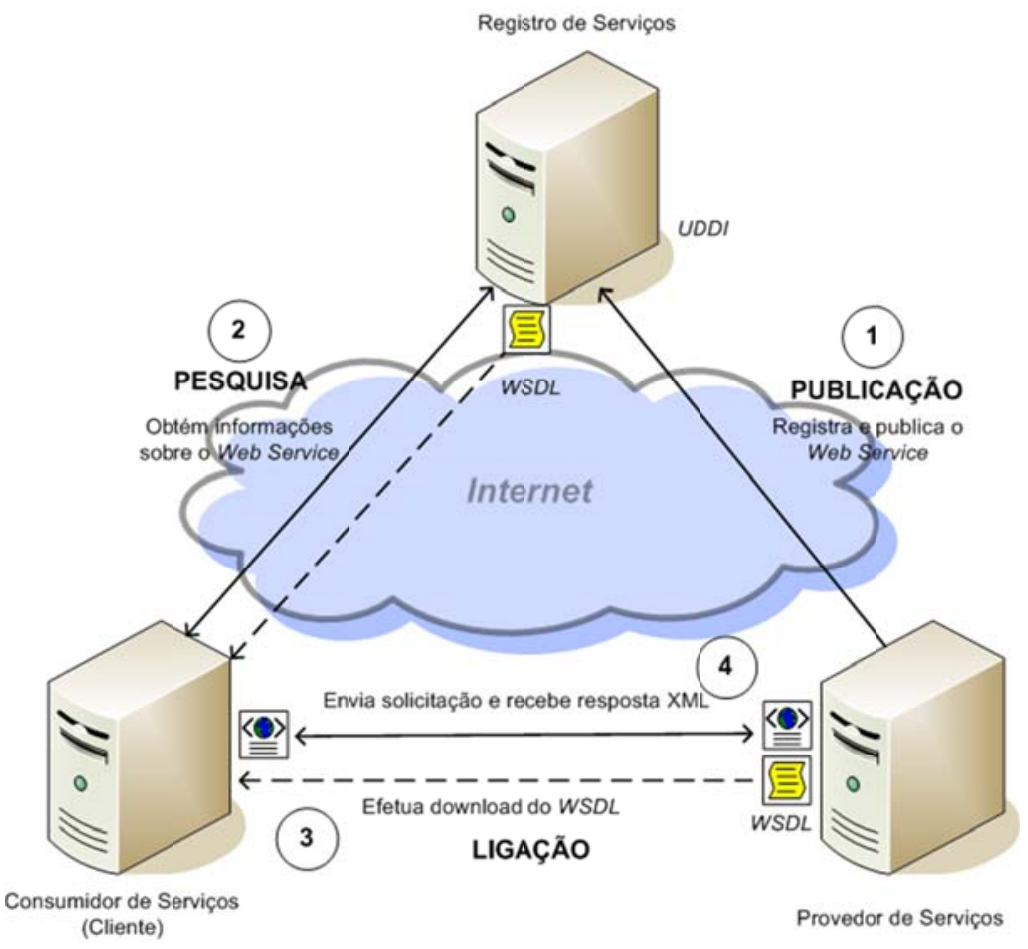

Figura 2.8 - Modelo de aplicação de Web Services. Adaptado de Gomes (2009). 
Sobre a arquitetura apresentada na figura 2.8 são realizadas três operações que definem um Web Service: Pesquisa (Discover), Publicação (Publish) e Ligação (Bind).

A Publicação da descrição de serviço disponibiliza informações no Servidor de Registro. Pois para que esse Web Service seja acessado por algum cliente seu $W S D L$ deve estar disponível. Essa operação está marcada pelo item 1 da figura 2.8.

$\mathrm{Na}$ Pesquisa, o Consumidor de Serviços obtém a descrição do serviço consultando o Registro dos Serviços. Este registro é realizado através de um serviço de registros padrão, o UDDI, que armazena os detalhes dos Web Services disponibilizados, como descrito através da operação 2 da figura 2.8. Em alguns casos de Web Services, a figura do UDDI não existe, o que permite a comunicação entre o cliente e o provedor sem intermediações.

Por último, na Ligação o Consumidor de Serviços invoca a interação com o serviço em tempo de execução a partir dos detalhes obtidos na descrição do serviço, em outras palavras, o Uniform Resource Identifier (URI). Assim o Consumidor obtém o arquivo WSDL (operação 3) e, em seguida, efetiva a utilização do $W e b$ Service desejado. Essa operação é realizada através do protocolo padrão de aplicações $S O A P$ que permite a troca de informações entre as aplicações através da linguagem $X M L$, representada pela operação 4 da figura 2.8 .

Na troca de informações, o cliente faz chamadas ao Web Service através do seu URI. Nessas chamadas há o envio de solicitações no formato XML. Ao receber essa requisição o Web Service responde ao cliente também através do mesmo formato.

\subsubsection{Camadas de Web Services}

As operações de Publicação, Pesquisa e Ligação podem ser representadas através de uma pilha de camadas demonstrada pela figura 2.9. Nesse diagrama ficam destacados os serviços e inter-relacionamentos estabelecidos por um conjunto de protocolos e linguagens. 
A camada de transporte define os mecanismos de comunicação responsáveis pelo transporte de dados entre os aplicativos. São exemplos de tecnologias usados nessa camada: Hypertext Transfer Protocol (HTTP), File Transfer Protocol (FTP) e Simple Mail Transport Protocol (SMTP).

\begin{tabular}{|c|c|}
\hline UDDI & $\begin{array}{c}\text { Publicação/ } \\
\text { Pesquisa dos Serviços }\end{array}$ \\
\hline WSDL & Descrição dos Serviços \\
\hline SOAP & Mensagens XML \\
\hline $\begin{array}{l}\text { HTTP, FTP, } \\
\text { E-MAIL, } \\
\text { IIOP, etc. }\end{array}$ & Rede de Transporte \\
\hline
\end{tabular}

Figura 2.9 - Estrutura em camadas de um Web Service.

A camada de mensagens $X M L$ corresponde ao modo de transferência de informação. Através da padronização da semântica das mensagens por meio da linguagem $X M L$ é possível definir um mecanismo interoperável para troca de mensagens em Web Services. O protocolo SOAP, tecnologia dessa camada, define o padrão de codificação para a troca de mensagens entre aplicações, independentemente do sistema operacional e linguagem de programação.

A Descrição de Serviços fornece um mecanismo ao Provedor de Serviços a fim de descrever as funcionalidades proporcionadas pelos Web Services. Nessa camada é utilizada a descrição WSDL para detalhar todas as características de cada chamada ou operação. Entre suas funcionalidades estão: a definição dos métodos presentes no serviço e seus parâmetros de entrada e saída.

Por último, as informações disponíveis no Web Service são publicadas pelo Provedor de Serviços em um registro central, disponível aos Consumidores de Serviços. O registro central, responsável por armazenar serviços e informações relacionadas ao negócio, é realizado através do padrão UDDI. Nesse diretório, os 
Provedores e Consumidores dos Serviços publicam e recuperam as informações desejadas.

\subsubsection{Tecnologias de Web Services}

Para melhor compreender as tecnologias que estruturam o funcionamento dos Web Services, faz-se uma breve descrição dos conceitos de $X M L, S O A P, W S D L$ e $U D D I$.

\subsubsection{XML}

$X M L$ é uma linguagem de marcação de dados (meta-markup language) (OLIVEIRA, 2005). O XML possibilita a definição de um número infinito de marcadores ou delimitadores (tags). Em contraponto à linguagem descritiva $H T M L$, cujos marcadores são utilizados para definir a formatação de caracteres e parágrafos, no $X M L$ é possível criar um sistema para dados estruturados.

Pode-se exemplificar uma estrutura de dados $X M L$ a partir de uma identificação de um sensor:

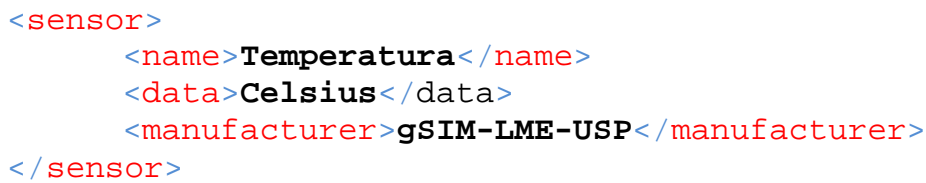

Esse exemplo apresenta a descrição de um sensor de temperatura, que transmite seus dados na escala "Celsius" e possui como fabricante o grupo "gSIMLME-USP”. Dessa forma, é confirmada a facilidade da utilização da linguagem $X M L$ para descrição e transmissão de informações. 


\subsubsection{SOAP}

SOAP é um protocolo que possibilita a comunicação entre Web Services. Esse padrão permite a troca de informação e dados através de objetos criados em diversas linguagens de programação. Esse mecanismo cria um ambiente de processamento distribuído e descentralizado (MITRA; LAFON, 2009).

O servidor $S O A P$ tem a habilidade de construir e analisar mensagens, além de transmiti-las através da rede. Como foi brevemente falado no subcapítulo anterior, a aplicação do cliente invoca a operação do Web Service, que interage, ao mesmo tempo, com um protocolo de dados subjacente como, por exemplo, o HTTP. Ao chegar ao servidor $S O A P$, a mensagem é convertida em objetos de uma linguagem de programação, na qual está escrito o Web Service. O programa, então, executa o serviço e envia a reposta ao servidor $S O A P$, que codifica e redireciona ao cliente do serviço (KREGER, 2001).

Uma mensagem SOAP é composta por: um elemento de identificação (Envelope), um cabeçalho opcional com informações processáveis pelos nós intermediários (Header), um corpo com a mensagem a ser transmitida (Body) e um elemento de informação sobre falhas de processamento de mensagens (Fault). O $S O A P$ define uma convenção para representar requisições e respostas. O corpo de uma mensagem de requisição inclui uma chamada de procedimento, especificando o nome do procedimento a ser invocado e seus parâmetros de entrada. Enquanto que o corpo da mensagem de resposta inclui o resultado e os parâmetros de saída.

\subsubsection{WSDL}

O WSDL é um formato $X M L$ que proporciona uma descrição do Web Service processável por máquina (CHINNICI et al., 2010). Um documento WSDL além de descrever a funcionalidade, provê um ponto de acesso para Consumidores de Serviços. Uma descrição WSDL completa inclui dois tipos de informação: uma interface de serviço e detalhes da descrição de serviço. 
A interface compreende um conjunto de operações realizadas por um serviço. Essa operação se inicia através de mensagens abstratas, que posteriormente são associadas a um protocolo de comunicação. É a partir das mensagens recebidas e enviadas por um Web Service que o serviço é descrito em WSDL.

A parte abstrata de uma descrição de serviço WSDL compreende:

- portType: que consiste em uma coleção de operações de Web Services.

-operation: define a funcionalidade de troca de mensagens.

- message: representa os dados enviados em uma transmissão de mensagem.

•types: representa os tipos de dados empregados pelo serviço.

Os elementos WSDL que compõem a parte concreta de uma descrição de serviço são:

- binding: especifica o estilo de interação e o protocolo de comunicações para um elemento portType.

- port: combina informações de ligação com um ponto de acesso especificado por uma $U R L$.

- service: conjunto de elementos port.

\subsubsection{UDDI}

A $U D D I$ disponibiliza um repositório de descrições de Web Services. Esse padrão oferece aos Consumidores de Serviços um mecanismo para localizar provedores, serviços e informações para acesso. Essa infraestrutura integra tanto a informação em ambiente de Web Services como os serviços expostos internamente.

Um diretório UDDI compreende dados sobre os seguintes itens: o Provedor de Serviços, a especificação de serviço e a implementação de serviço. Um mapeamento entre elementos $W S D L$ e elementos $U D D I$ para a publicação de serviços é demonstrado na tabela 2.1 (OLIVEIRA, 2005). 
Tabela 2.1 - Mapeamento entre elementos $W S D L$ e elementos $U D D I$.

\begin{tabular}{ll}
\hline Elemento WSDL & Elemento UDDI \\
\hline service & businessService \\
port e binding & bindingTemplate \\
portType & $t$ Model \\
binding & $t$ Model \\
\hline
\end{tabular}

O elemento businessService contém o nome do serviço especificado. Também inclui o identificador do provedor do serviço e um elemento de bindingTemplate, que contém informações sobre a implementação do serviço. $\mathrm{O}$ emprego do tModel permite a utilização do UDDI para representar dados e metadados sobre serviços e entidades. A partir da tabela 2.1, mostra-se que as referências de portType e binding são armazenadas nessa estrutura.

\subsection{ANATOMIA E FISIOLOGIA DO SISTEMA CARDIOVASCULAR}

O sistema cardiovascular humano é composto pelo coração, vasos arteriais, sistema tubular trocador, vasos venosos e vasos linfáticos. Sua principal função é a de fornecimento do fluxo sanguíneo, de modo a garantir a condução e a distribuição de nutrientes e oxigênio $\left(\mathrm{O}_{2}\right)$, além da remoção de resíduos do metabolismo celular e de gás carbônico $\left(\mathrm{CO}_{2}\right)$ dos tecidos do organismo humano.

Localizado obliquamente no mediastino médio na cavidade torácica, o coração humano é o órgão responsável pelo bombeamento de sangue para todo o corpo. O coração é dividido em quatro câmaras, as duas superiores são os átrios e as duas inferiores são os ventrículos. Os átrios recebem o sangue que flui das veias. $\mathrm{O}$ átrio direito recebe o sangue venoso trazido pela veia cava superior e inferior. Já o átrio esquerdo recebe o sangue oxigenado dos pulmões através das veias pulmonares (GUYTON; HALL, 2006).

Os átrios dão acesso aos ventrículos, que são câmaras expulsoras capazes de impulsionar o sangue através dos pulmões, no caso do ventrículo direito, e do 
sistema circulatório periférico, no caso do ventrículo esquerdo. De forma a manter o fluxo sanguíneo através do sistema cardiovascular.

\subsubsection{Eletrofisiologia do coração}

Uma característica importante da musculatura cardíaca é que ela é eletrogênica, podendo gerar potenciais de ação em qualquer de suas células. O ritmo intrínseco espontâneo gerado pelas células especializadas dos nódulos sinoatrial (SA) e atrioventricular (AV) determinam a atividade de marca-passo cardíaca. No coração, figura 2.10, esse estímulo se origina no nódulo SA e se propaga na forma de ondas elétricas. Esse estímulo se difunde até o nódulo AV, que o retém durante um período de tempo, possibilitando que o sangue ejetado pelos átrios penetre nos ventrículos através das valvas AV.

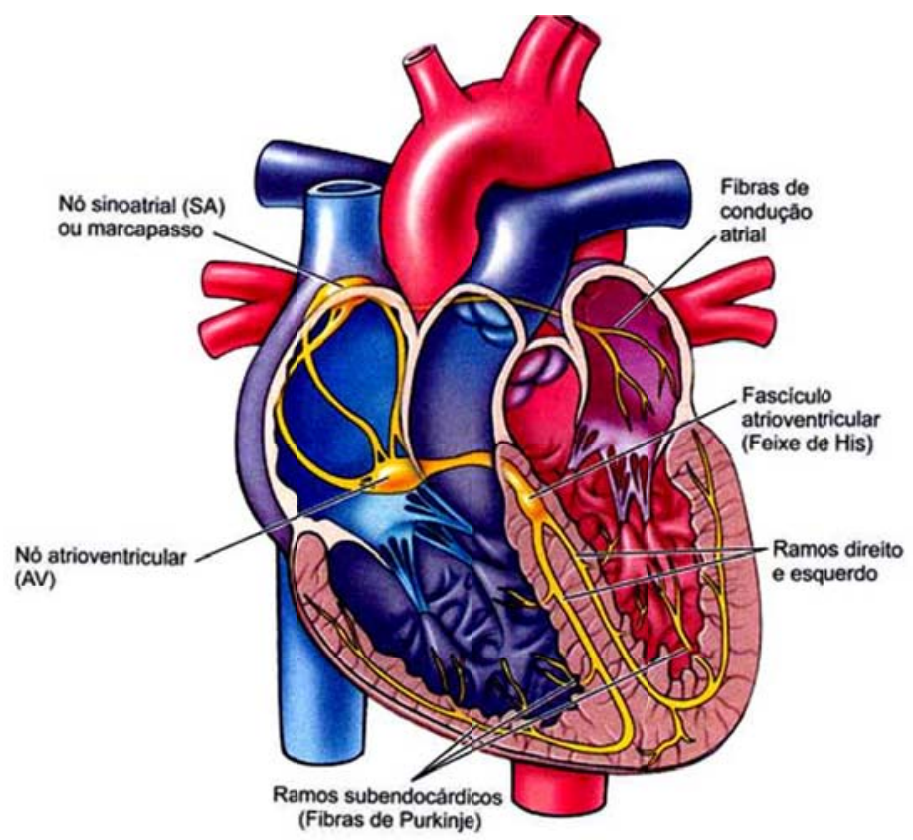

Figura 2.10 - Detalhe das câmaras do coração. Adaptado de Guyton e Hall (2006).

Após a pausa, o estímulo segue pelo feixe de His e seus ramos direito e esquerdo, terminados nas fibras de Purkinje. Essas fibras, por sua vez, estando interligadas às células miocárdicas, realizam a comunicação e controle do 
bombeamento cardíaco através do movimento de contração (GUYTON; HALL, 2006).

Todo o processo de bombeamento tem como origem as correntes eletrolíticas de íons de sódio $\left(\mathrm{Na}^{+}\right)$, de cálcio $\left(\mathrm{Ca}^{2+}\right)$ e de potássio $\left(\mathrm{K}^{+}\right)$que geram ciclos de despolarização e repolarização das células miocárdicas. Essas na fase de repouso apresentam cargas positivas no seu lado externo e cargas negativas no seu interior. Ao receber um estímulo elétrico as cargas negativas tornam-se positivas. $\mathrm{O}$ efeito dessa despolarização é a contração da fibra miocárdica. Por outro lado, a repolarização restabelece o estado original das cargas negativas no interior das células, restabelecendo a possibilidade das fibras se contraírem novamente.

\subsubsection{Eletrocardiograma: medidas, monitorização e diagnóstico}

Todos os impulsos elétricos que surgem na estimulação do coração e nas fases de repouso e recuperação podem ser registrados através do eletrocardiograma. A medida desse parâmetro fisiológico teve início com Willem Einthoven, cujo trabalho foi desenvolver um monitor não invasivo a partir de um galvanômetro de corda. Com esse instrumento Einthoven foi capaz de medir diferenças de potencial entre as extremidades do corpo humano imersas em soluções salinas. Desde então o registro do eletrocardiograma permitiu monitorar o funcionamento do coração e realizar o diagnóstico de cardiopatias, distúrbios ou estados mórbidos.

Segundo Guyton e Hall (2006) o eletrocardiograma normal é composto pela onda P, pelo complexo QRS e pela onda T, como pode ser visto na figura 2.11. Essas formas de onda que representam os impulsos elétricos ao longo do coração estão ligadas aos diferentes eventos mecânicos da atividade cardíaca.

A onda P representa o potencial elétrico gerado pela despolarização do átrio direito. Essa curva marca o início do batimento cardíaco. Após a onda P há um pequeno isoelétrico que corresponde ao atraso do nó atrioventricular. Nesse mesmo sinal, o complexo QRS está relacionado à despolarização dos ventrículos e ao início 
da contração ventricular. Por último, a onda $\mathrm{T}$ é decorrente da repolarização ventricular, que permite aos ventrículos se recuperarem do estado de despolarização.

O eletrocardiograma normal, quando medido através de eletrodos nos dois braços, à maneira de Einthoven, possui forma de onda similar à apresentada na figura 2.11. Nesse sinal, nota-se que a tensão do complexo QRS se aproxima de $1 \mathrm{mV}$ do pico da onda $\mathrm{R}$ até o vale da onda $\mathrm{S}$, de 0,1 a $0,3 \mathrm{mV}$ do pico da onda $\mathrm{P}$ até a referência e de 0,2 a $0,3 \mathrm{mV}$ do pico da onda $\mathrm{T}$ até a referência.

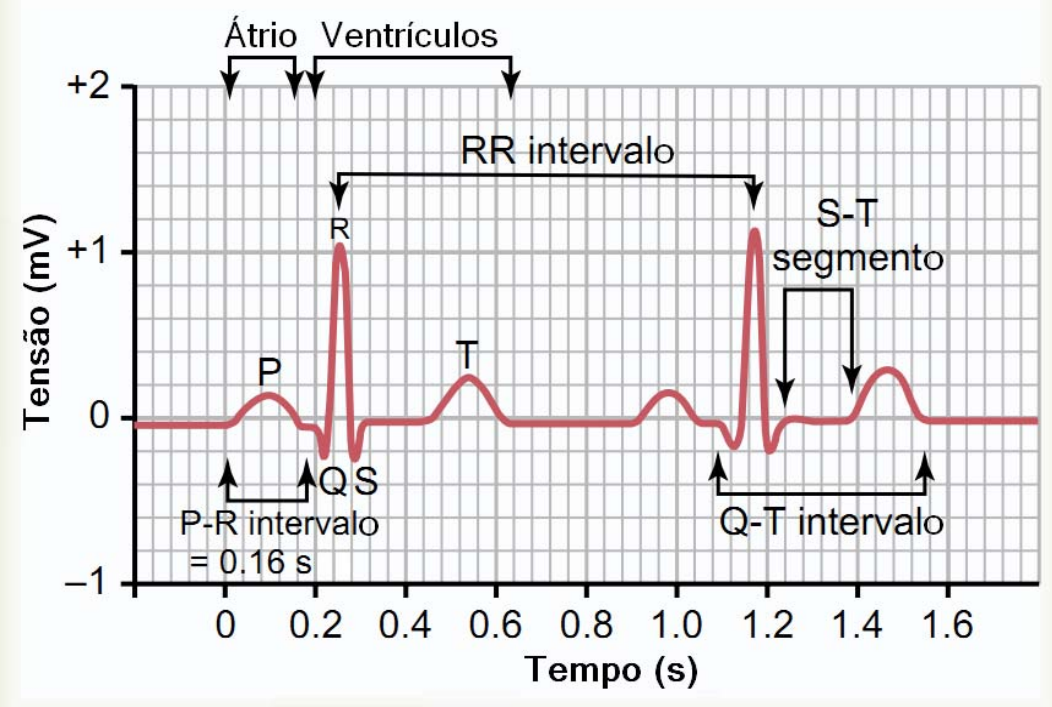

Figura 2.11 - Eletrocardiograma normal. Adaptado de Guyton e Hall (2006).

Outra medida que pode ser inferida é a frequência cardíaca. Essa frequência corresponde ao intervalo temporal entre dois batimentos sucessivos. Em um eletrocardiograma normal esse tempo é de aproximadamente $0,83 \mathrm{~s}$, o que corresponde a 72 batimentos por minuto. Desse tempo total, a onda P corresponde a aproximadamente $0,1 \mathrm{~s}$, valor esse que varia com a idade. O intervalo P-R pode variar entre 0,12 a $0,2 \mathrm{~s}$, assim como o complexo QRS entre 0,06 a $0,1 \mathrm{~s}$. Ao final do sinal o intervalo Q-T tem aproximadamente 0,35s (GUYTON; HALL, 2006). 
Outra característica do sinal de eletrocardiograma decorre da sua análise espectral no qual se pode analisar valores significativos dentro da faixa de $0,01 \mathrm{~Hz}$ à $250 \mathrm{~Hz}$. Essa banda é utilizada, principalmente, em instrumentos de diagnóstico médico (BAILEY; BERSON; GARSON, 1990). Em equipamentos de simples monitoração a faixa de frequência amostrada fica entre $0,5 \mathrm{~Hz}$ e $50 \mathrm{~Hz}$, sendo realçada as frequências próximas a $17 \mathrm{~Hz}$, que indicam o conteúdo do pico da onda $\mathrm{R}$.

Dentro dos diagnósticos factíveis com a análise do eletrocardiograma podem ser citados: variações do ritmo cardíaco, como, por exemplo, batimentos irregulares causados por arritmias. Também são verificáveis extra-sístoles (impulsos elétricos que aparecem antes do tempo), pausas (batimento de escape e parada sinusal), taquicardias (ritmo rápido regular ou irregular), bloqueios (no nó sinusal, no nódulo $\mathrm{AV}$, e nos ramos do feixe de His), entre outros.

\subsubsection{Derivações eletrocardiográficas}

Os sinais de um eletrocardiograma podem ser obtidos através de eletrodos não invasivos, ou de superfície. Esses eletrodos podem ser ativos ou passivos. Para eletrocardiografia são utilizados, especialmente, eletrodos passivos revestidos com cloreto de prata $(\mathrm{Ag}-\mathrm{AgCl})$, que possuem sensibilidade para sinais de até $500 \mathrm{~Hz}$ com tensões entre $50 \mu \mathrm{V}$ a $5 \mathrm{mV}$ (ORTOLAN, 2002).

Os eletrodos são posicionados no paciente de acordo com as derivações eletrocardiográficas. Essas derivações possibilitam verificar o impulso elétrico em determinadas direções e sentidos. Além disso, são definidas a partir da posição dos eletrodos, que podem estar no plano frontal, a constituir as derivações periféricas, ou no plano horizontal (transversal) formando as derivações precordiais. Os eletrocardiógrafos podem registrar 1, 3, 6 ou até as 12 derivações de uma só vez.

As derivações periféricas são realizadas através de um eletrodo no braço direito, outro no braço esquerdo e, finalmente, na perna esquerda. As derivações periféricas bipolares são constituídas da derivação D1, com um eletrodo no braço direito (pólo negativo) e outro no braço esquerdo (pólo positivo); da derivação D2, 
com um eletrodo no punho direito (pólo negativo) e outro no tornozelo esquerdo (pólo positivo); e da derivação D3, com um eletrodo no punho esquerdo (pólo negativo) e outro no tornozelo esquerdo (pólo positivo). Essas derivações podem ser vistas na figura 2.12 .
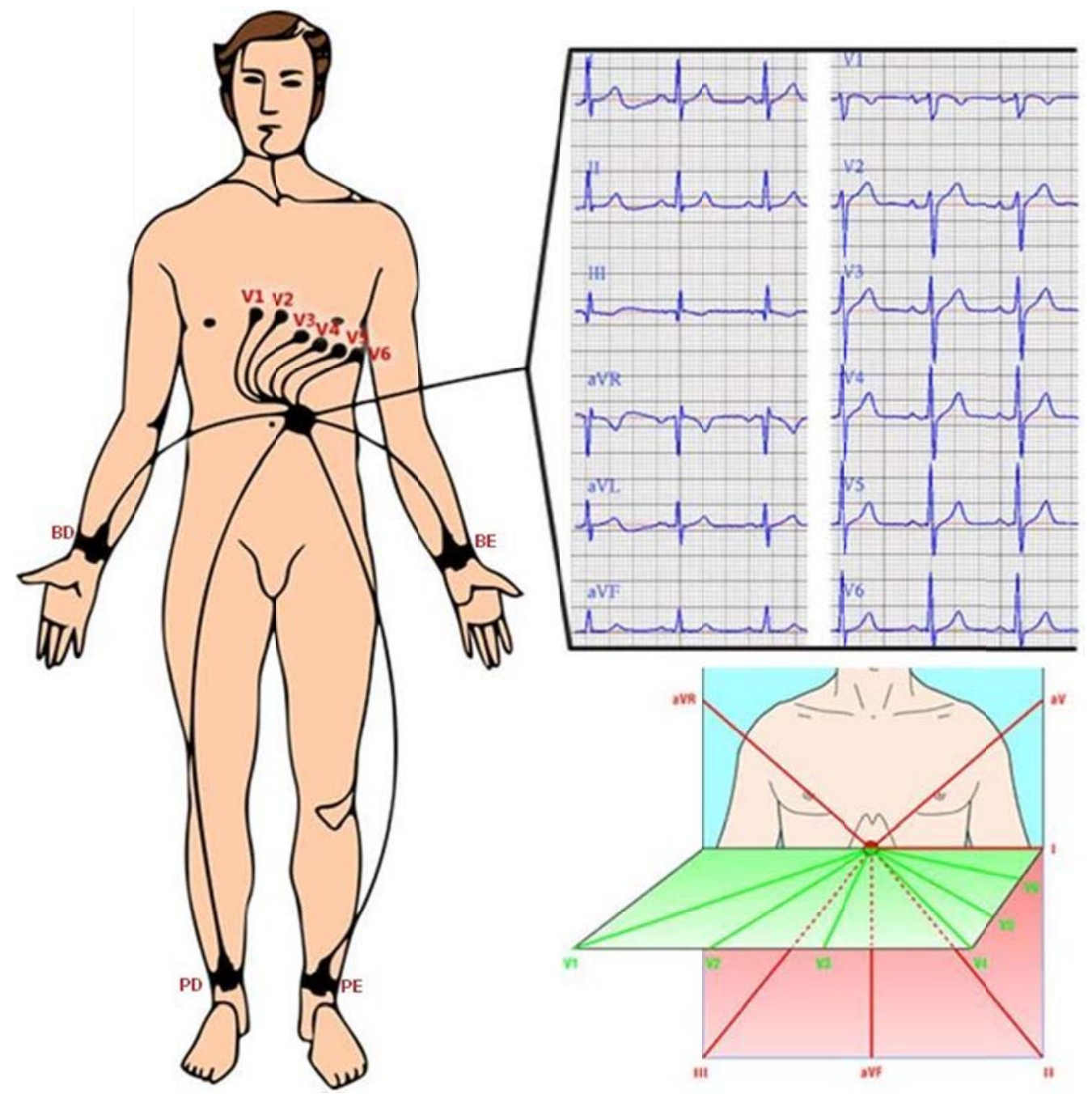

Figura 2.12 - Derivações eletrocardiográficas (IVLINE, 2011).

As derivações periféricas unipolares também são realizadas com eletrodos nos membros, contudo dois eletrodos são ligados ao terminal negativo do eletrocardiógrafo. O terceiro eletrodo, terminal positivo, é conectado ao braço 
direito, no caso da derivação aVR; no braço esquerdo, na derivação aVL; e na perna esquerda, na derivação aVF.

Já as derivações precordiais são realizadas através de seis eletrodos positivos que se fixam sobre o tórax do paciente. Os eletrodos $V_{1}, V_{2}, V_{3}, V_{4}, V_{5}$ e $V_{6}$ correspondem a pontos bem definidos da topografia do coração. Através de cada derivação são medidos os potenciais elétricos da musculatura cardíaca imediatamente abaixo do eletrodo (GUYTON; HALL, 2006). A figura 2.12 apresenta as doze derivações do eletrocardiograma padrão.

\subsection{FISIOLOGIA ARTICULAR}

A articulação humana é constituída pela coaptação de dois ou mais ossos com o auxílio de músculos esqueléticos, por ligamentos e pela cápsula articular. A partir dessa estrutura o ser humano é capaz de movimentar e articular seus membros em diferentes ângulos e graus de liberdades. Um parâmetro para avaliar a dinâmica desse movimento é a Amplitude de Movimento (ADM). Através dessa medida é possível acompanhar a reabilitação funcional dos movimentos ativos e passivos de um membro.

Tal acompanhamento auxilia nos processos de reabilitação fisioterápicos conhecidos como cinesioterapia ${ }^{6}$. Além disso, permite analisar os movimentos durante práticas esportivas. Para tanto são utilizados goniômetros como instrumentos de medição de ADM. Esses equipamentos medem a amplitude angular ou inclinação realizada por cada articulação, de modo a informar quantitativamente a evolução de um tratamento em fisioterapia, ou a melhoria do desempenho de atletas.

Em especial, a medida de amplitude de movimento dos membros superiores é de extrema relevância na recuperação de doenças articulares, musculares ou sistêmicas; agressões traumáticas ou cirúrgicas; e imobilização ou inatividade. São exemplos dessas doenças a osteoartrite, a bursite e a tendinite (SOARES et al., 2010).

\footnotetext{
${ }^{6}$ Cinesioterapia (do grego kinesis, movimento e therapeia, terapia).
} 


\subsubsection{Membro superior}

O membro superior é formado pelo braço, antebraço, punho e mão. O braço é constituído pelo úmero, que se articula no cotovelo com os ossos do antebraço. O antebraço, por sua vez, é formado por dois ossos: o rádio e a ulna. A mão é constituída pelos ossos do carpo, que formam o punho; pelo metacarpo, que forma a palma da mão; e pelos dedos.

Em fisioterapia os movimentos são classificados em ativos ou passivos. No primeiro caso, os movimentos ocorrem por contração dos músculos que cruzam a articulação, já no segundo, a contração ocorre devido a uma força externa aplicada. Os principais tipos de movimentos realizados são: flexão, extensão, rotação, adução e abdução.

No membro superior esses movimentos ocorrerem no ombro, cotovelo e punho. O ombro é a articulação que possui maior mobilidade, pois apresenta quatro graus de liberdade. Através dessa mobilidade o ombro permite os movimentos de flexão, extensão, adução e abdução. Esses movimentos estão na figura 2.13.

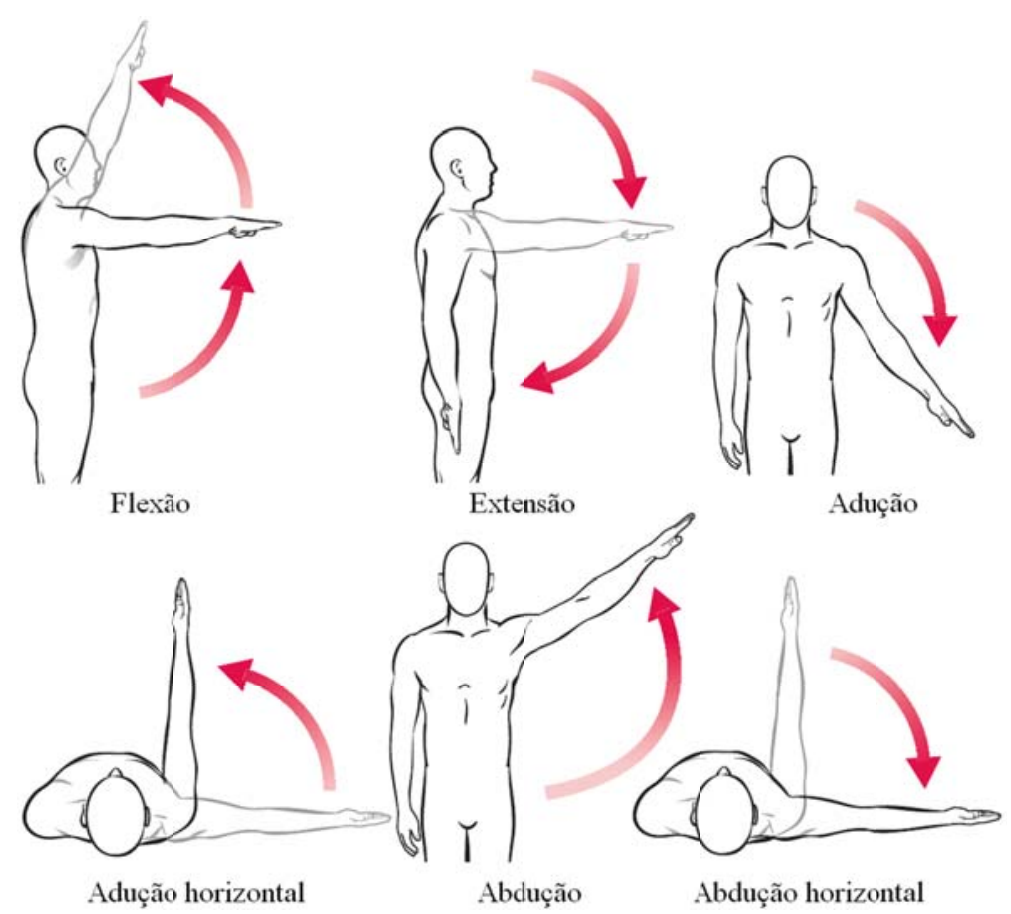

Figura 2.13 - Movimentos realizados pelo ombro. Adaptado de Lippert (2006). 
Já o cotovelo permite duas articulações: a flexão-extensão e a pronosupinação, como apresentado na figura 2.14. Na fisiologia articular o movimento de levar o antebraço para frente é chamado flexão e o movimento de levar o antebraço para trás é denominado extensão. A amplitude desses movimentos varia, tipicamente, entre 20 e $180^{\circ}$. A prono-supinação do cotovelo corresponde ao movimento de rotação do antebraço ao redor de seu eixo longitudinal (KAPANDJI, 2007).

Os movimentos de prono-supinação devem ser avaliados tendo como referência o cotovelo flexionado a $90^{\circ}$ e o braço próximo ao corpo. A supinação ocorre quando a palma da mão está voltada para cima e o polegar é direcionado para fora, sua amplitude é de $90^{\circ}$. Enquanto isso, a pronação ocorre quando a palma da mão está direcionada para baixo e o polegar voltado para dentro, sua amplitude angular é de aproximadamente $85^{\circ}$ (SOARES et al., 2010). A figura 2.14 representa esquematicamente os movimentos (KAPANDJI, 2007).

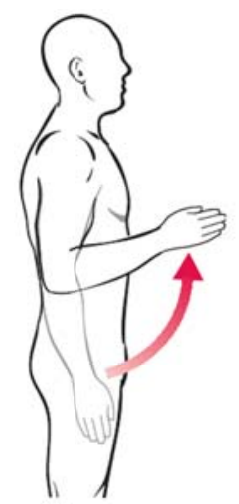

Flexão

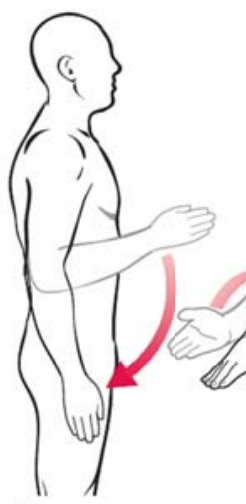

Extensão
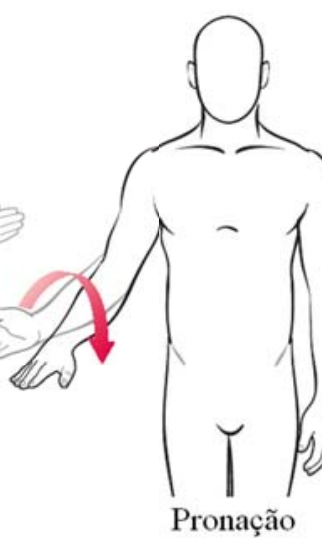

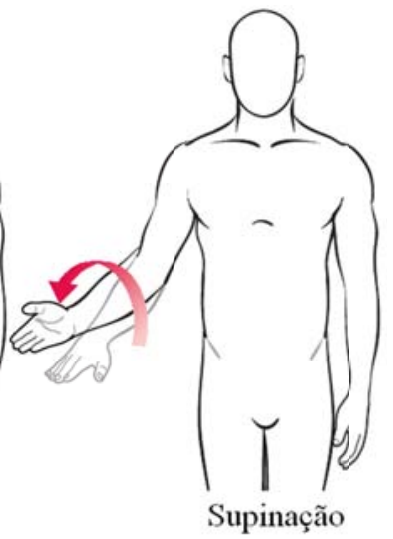

Figura 2.14 - Movimentos realizados pelo cotovelo. Adaptado de Lippert (2006).

Ainda nos membros superiores, destaca-se o complexo do punho. Esse possui quatro tipos de movimentos: flexão e extensão do punho, além do desvio ulnar e desvio radial, todos esses representados pela figura 2.15 . 


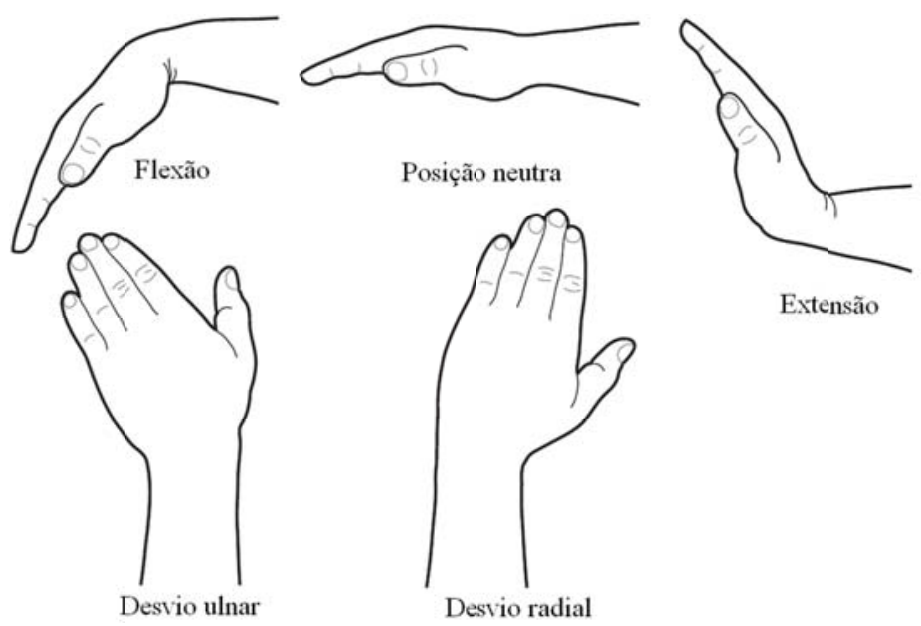

Figura 2.15 - Movimentos realizados pelo punho. Adaptado de Lippert (2006).

Para melhorar a mensuração desses movimentos, atualmente, são utilizados goniômetros eletrônicos. Nesses a medida de ADM é convertida em um sinal elétrico por meio de um transdutor, que pode ser um extensômetro, acelerômetro, ou potenciômetro, entre outros. A utilização de eletrogoniômetros minimiza erros sistemáticos, principalmente, relacionados ao posicionamento do instrumento no paciente (ELVERU et al., 1988) (SOARES et al., 2010). 


\section{DESENVOLVIMENTO DOS NÓS BIOMÉDICOS UTILIZANDO O PROTOCOLO IEEE1451}

Nesse capítulo é apresentada a arquitetura da rede de sensores biomédicos utilizando o protocolo IEEE1451. Para tanto são demonstrados: o projeto de um circuito condicionador de biopotenciais, a seleção de acelerômetros para medição de amplitude de movimento, a definição dos sistemas embarcados microcontrolados, além do estudo, adaptação e seleção de funcionalidades da norma IEEE1451 e sua interface com a Internet. Com isso são delimitados os materiais e métodos utilizados no desenvolvimento e integração do projeto em questão.

\subsection{Arquitetura da rede de sensores}

A priori, a arquitetura da rede de sensores biomédicos IEEE1451 foi dividida em três etapas: o projeto do sensoriamento de sinais de eletrocardiografia (ECG) e de ADM, que representam as aplicações de engenharia biomédica; o sistema embarcado microcontrolado que realizou a aquisição e o condicionamento de sinais, além de efetuar a interface entre sensores e o computador local através do protocolo IEEE1451; por último, a disponibilização dos dados coletados através de um Web Service. Essa configuração está apresentada na figura 3.1.

Da arquitetura proposta na figura 3.1, primeiramente, verificam-se duas aplicações para desenvolvimento da rede de sensores IEEE1451. A primeira um circuito de aquisição de biopotenciais. Nessa aplicação foi amostrado o sinal de ECG e em seguida calculou-se o valor da frequência cardíaca do paciente. A outra aplicação está ligada à utilização de inclinômetros para acompanhamento médico no tratamento fisioterápico. Esse sensor realizou medidas de extensão e flexão do cotovelo, através de dois acelerômetros alocados junto ao braço e antebraço do paciente.

A aquisição de sinais de ECG foi executada por um microcontrolador $A V R$ ATmega8535 (ANEXO A). Esse microprocessador adicionado de periféricos e 
memórias, além de amostrar o sinal, efetuou seu condicionamento e processamento. A transmissão do sinal a um computador local foi implementada por uma rede Wifi (IEEE802.11b/g) através do módulo WiFly GSX (ANEXO B). O grupo formado pelo sensor, microcontrolador e interface sem fio definiu um nó WTIM. Nesse módulo foram desenvolvidos e integrados os protocolos IEEE1451.0 e IEEE1451.5 (NEMETH-JOHANNES, 2007) (SONG; LEE, 2006).

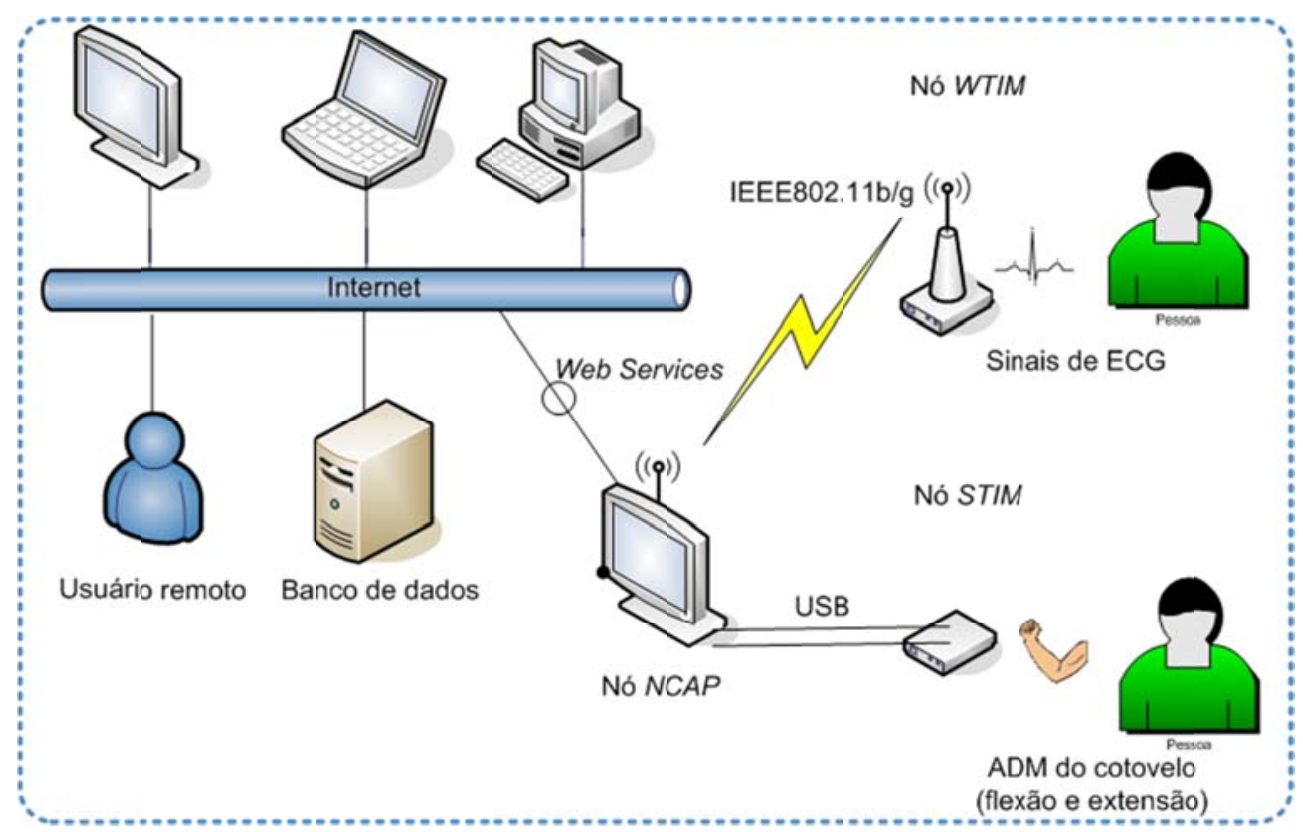

Figura 3.1 - Arquitetura da rede de sensores biomédicos utilizando o protocolo IEEE1451.

A segunda aplicação em engenharia biomédica permitiu monitorar a amplitude de movimento realizada pelas articulações do corpo humano. Isso foi possível através da medida de dois acelerômetros Memsic 2125 (ANEXO D). Os sinais desses sensores foram medidos por um microcontrolador AT90USB162 (ANEXO C), que por sua vez executou o condicionamento e a transmissão dos dados por uma interface USB. Esse nó, composto de sensores e sistema embarcado constituiu o STIM. Nesse mesmo circuito foram programados os protocolos IEEE1451.0 e IEEE1451.2 (SONG; LEE, 2008b). 
Dentro da arquitetura proposta, o computador que recebeu tanto os dados do nó $W T I M$ como os do STIM possuiu a função de $N C A P$. Nesse nó foram projetados os protocolos IEEE1451.0, IEEE1451.2 e IEEE1451.5. Ao final do projeto, os dados dos sensores puderam ser visualizados localmente ou remotamente através de um Web Service implementado com o framework Apache Axis2.

\subsection{Condicionamento de biopotenciais}

Biopotenciais são potenciais elétricos resultantes dos processos eletroquímicos celulares. Os principais biopotenciais, segundo Webster (1998), são o eletrocardiograma, o eletromiograma e o eletroencefalograma ${ }^{7}$. Como já apresentado, os sinais do ECG estão ligados aos estímulos que originam as contrações do músculo cardíaco. Já os sinais de eletromiografia (EMG) refletem a atividade das fibras musculares e os sinais de eletroencefalografia (EEG) resultam da atividade conjunta de grupos de células nervosas.

O registro e a análise de biopotenciais possibilitam o diagnóstico de doenças e a monitoração durante incursões cirúrgicas. Tal medida não requer cortes de tecidos, nem procedimentos cirúrgicos, somente demanda o correto posicionamento de eletrodos não invasivos, associados a uma instrumentação dedicada de amplificação, filtragem e condicionamento dos sinais. Para realizar esse estágio é necessário conhecer as magnitudes típicas dos biopotenciais, em especial do ECG, objeto de estudo desse trabalho.

Tabela 3.1 - Magnitudes de biopotenciais (WEBSTER, 1998).

\begin{tabular}{ll}
\hline Tipo de sinal & Características do sinal \\
\hline ECG & Tensão: $100 \mu \mathrm{V}$ a $4 \mathrm{mV}$ \\
Eletrocardiograma & Largura de banda: $0,01 \mathrm{~Hz}$ a $250 \mathrm{~Hz}$ \\
\hline EMG & Tensão: $100 \mu \mathrm{V}$ a $5 \mathrm{mV}$ \\
Eletromiograma & Largura de banda: $0 \mathrm{~Hz}$ a $2,5 \mathrm{kHz}$ \\
\hline EEG & Tensão: $5 \mu \mathrm{V}$ a $300 \mu \mathrm{V}$ \\
Eletroencefalograma & Largura de banda: $0 \mathrm{~Hz}$ a $150 \mathrm{~Hz}$ \\
\hline
\end{tabular}

\footnotetext{
${ }^{7} \mathrm{O}$ autor também cita, secundariamente, o eletroneurograma (ENG) e o eletroretinograma (ERG).
} 
A tabela 3.1 mostra as características dos biopotenciais. Acerca dos três tipos de sinais apresentados, verifica-se que os sinais apresentam baixa amplitude (diferença de potencial de no máximo $5 \mathrm{mV}$ ) e faixa de frequências de até $2,5 \mathrm{kHz}$. Devido essas particularidades, a medida de biopotenciais é influenciada por diferentes tipos de ruídos.

As principais fontes são: a interferência do campo elétrico de $50 \mathrm{~Hz} / 60 \mathrm{~Hz}$ (conforme cada país) e suas harmônicas, provenientes da linha de transmissão de energia presente na maioria dos ambientes; a interferência eletromagnética injetada através do acoplamento capacitivo e/ou indutivo dos pares de eletrodos e dos fios; e o "artefato de movimento", sinal Direct Current $(D C)$, que se forma a partir das cargas estáticas entre eletrodos e o paciente (BRONZINO, 2000).

Essas informações servem como base para o desenvolvimento de um circuito condicionador de biopotenciais capaz, no escopo desse trabalho, de amplificar e filtrar sinais de ECG, com objetivo de obter o batimento cardíaco do paciente, minimizando a influência de interferências externas no sinal.

Embora focado na medida de ECG, esse sistema poderia também ser utilizado em engenharia de reabilitação através de interfaces homem-máquina por meio de sinais de EMG ou de EEG (VEGA, 2010) (GODOI, 2010).

\subsubsection{Arquitetura do circuito condicionador de biopotenciais}

Posto as dificuldades de tomar medidas de biopotenciais diretamente, faz-se necessário desenvolver um amplificador de biopotenciais com a finalidade de amplificar tensões para níveis adequados de processamento e aquisição digital (na casa de unidade de volts). Além disso, cabe ao circuito atenuar as interferências inerentes ao processo de medição, com o intuito de melhorar a relação sinal ruído. A figura 3.2 descreve em blocos os estágios que compõem o circuito condicionador projetado. 
As partes projetadas desse circuito, respectivamente, foram: um amplificador diferencial, um filtro passa-altas, um filtro rejeita-faixas, um filtro passa-baixas e dois estágios de amplificação. O desenvolvimento do circuito condicionador foi dividido em três etapas: o projeto do filtro, que se encarregou de tratar das topologias e equações acerca dos filtros; as simulações dos blocos e a caracterização do protótipo construído. A primeira parte é descrita no texto que se segue, enquanto que os resultados das simulações e caracterizações são detalhados nos subcapítulos $4.1 \mathrm{e}$ 4.2 .

Para a tomada de biopotenciais foi efetuada uma medida diferencial dos sinais provenientes de dois eletrodos devidamente alocados nos braços do paciente, utilizando uma derivação periférica bipolar D1. Nessa medida os sinais dos pólos positivo e negativo estão defasados e sofrem a influência de um ruído comum, que uma vez amplificado pode distorcer o sinal original.

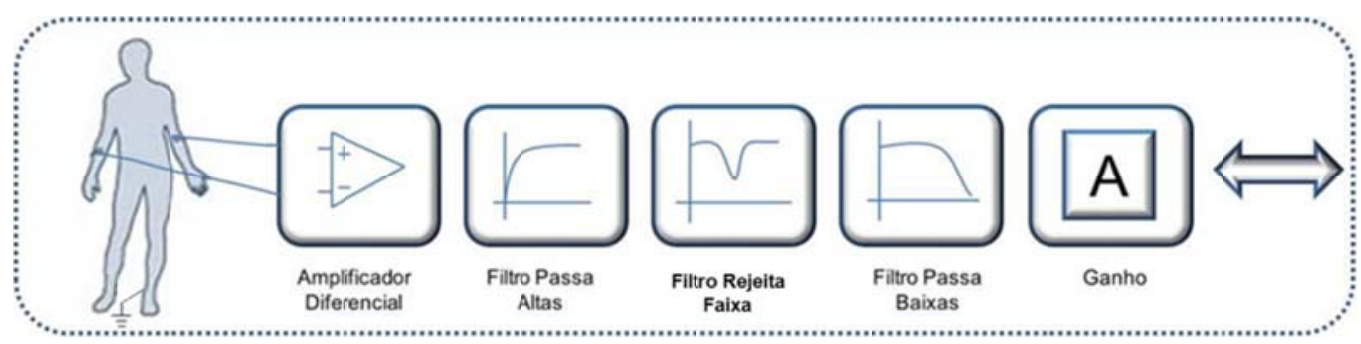

Figura 3.2 - Esquema de circuitos para condicionamento de biopotenciais.

Para atenuar a influência de ruídos foi utilizado um amplificador de instrumentação integrado, o AD620 da Analog Devices. Esse amplificador diferencial tem como característica uma alta taxa de rejeição em modo comum, ou CommonMode Rejection Ratio $(C M R R)^{8}$, que corresponde à eficiência do amplificador em rejeitar sinais idênticos aplicados, simultaneamente, em suas entradas (SEDRA; SMITH, 2007). Além disso, esse amplificador diferencial permite ganho ajustável de 1 a 1000 através de um único resistor. Esse ganho é expresso na eq.(3.1).

\footnotetext{
${ }^{8}$ Para o AD620 o valor de CMRR é de $100 \mathrm{~dB}$ para um ganho mínimo de 10 vezes.
} 


$$
A=\frac{49,4 k \Omega}{R_{G}}+1
$$

Para calcular um ganho (A) variável entre 2 e 1000 é necessário um $R_{G}$ entre os valores de $50 \Omega$ e $50 \mathrm{k} \Omega$, o que foi feito alocando nos terminais desse amplificador um potenciômetro multivoltas com resistência elétrica de $50 \mathrm{k} \Omega$ em série com uma resistência de $50 \Omega$.

Para os filtros analógicos ativos passa-altas e passa-baixas foi escolhido o projeto a partir da topologia Sallen-Key com aproximação Butterworth. Sendo o primeiro filtro de $2^{\mathrm{a}}$ ordem $^{9}$ e o segundo de $4^{\mathrm{a}}$ ordem, o que torna a transição da banda de passagem para a banda de rejeição mais inclinada nos filtros passa-baixas. A escolha por filtros Butterworth teve como objetivo garantir baixa ondulação em banda passante. Outros tipos de filtros como o Bessel e o Chebyshev priorizam resposta linear de fase e transição estreita, respectivamente (SEDRA; SMITH, 2007).

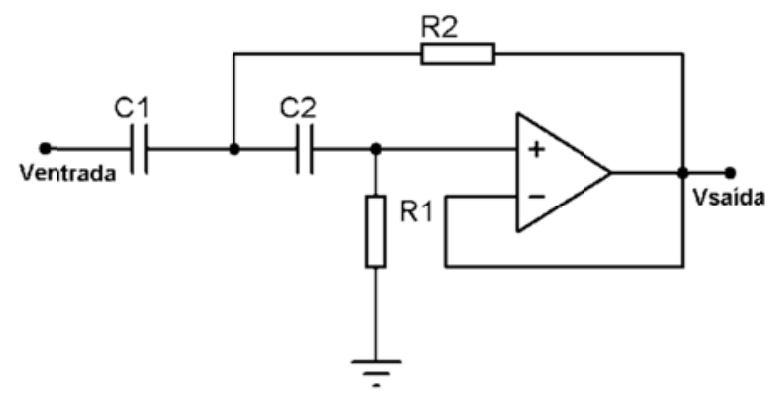

Figura 3.3 - Esquema do filtro Butterworth passa-altas de $2^{\mathrm{a}}$ ordem.

O projeto do filtro ativo Butterworth passa-altas de $2^{\mathrm{a}}$ ordem com frequência de corte $0,5 \mathrm{~Hz}$ tem como esquema o circuito apresentado na figura 3.3 (TEXAS, 2002). Dado que a função de transferência geral de filtros passa-altas é representada pela eq.(3.2).

$$
A(s)=\frac{A_{\infty}}{\prod_{i}\left(1+\frac{a_{i}}{s}+\frac{b_{i}}{s^{2}}\right)}
$$

\footnotetext{
${ }^{9}$ Para aproximação Butterworth a declividade do filtro na faixa de transição varia com $20 \mathrm{NdB} /$ década, no qual $\mathrm{N}$ corresponde a ordem do filtro.
} 
Tem-se para filtros de $2^{\mathrm{a}}$ ordem com topologia Sallen-Key e ganho unitário tal que $\mathrm{A}_{\infty}=1$ e $\mathrm{C}_{1}=\mathrm{C}_{2}=\mathrm{C}$ a form.(3.3).

$$
A(s)=\frac{1}{1+\frac{2}{\omega_{c} R_{1} C} \cdot \frac{1}{s}+\frac{2}{\omega_{c}^{2} R_{1} R_{2} C^{2}} \cdot \frac{1}{s^{2}}},
$$

A partir dessa fórmula são encontrados os coeficientes $a_{1}=\frac{2}{\omega_{c} R_{1} C} \mathrm{e}$ $b_{1}=\frac{1}{\omega_{c}^{2} R_{1} R_{2} C^{2}}$. Esses coeficientes são tabelados para cada tipo de filtro e ordem (TEXAS, 2002), em específico, para o filtro do tipo Butterworth de $2^{\mathrm{a}}$ ordem os coeficientes são dados por: $a_{1}=1,4142$ e $b_{1}=1$. Para $\mathrm{f}_{\mathrm{c}}=0,5 \mathrm{~Hz}$ e capacitância fixa igual a $470 \mathrm{nF}$, encontraram-se, aproximadamente $\mathrm{R}_{1}=820 \mathrm{k} \Omega$ e $\mathrm{R}_{2}=470 \mathrm{k} \Omega$. Esses valores foram aproximados para possibilitar a utilização de resistores comerciais.

$\mathrm{O}$ projeto do filtro ativo notch duplo $\mathrm{T}$ rejeita-faixas com rejeição em $60 \mathrm{~Hz}$ foi dado a partir do esquema elétrico apresentado na figura 3.4. Dentre as vantagens de se empregar essa topologia de filtro, é possível citar a utilização de resistores e capacitores, não demandando a presença de indutores e, além disso, a alta seletividade (Q), que representa um índice de mérito de filtros rejeita-faixas, pois quanto maior a seletividade, somente os sinais próximos da frequência de rejeição $\left(f_{o}\right)$ serão atenuados.

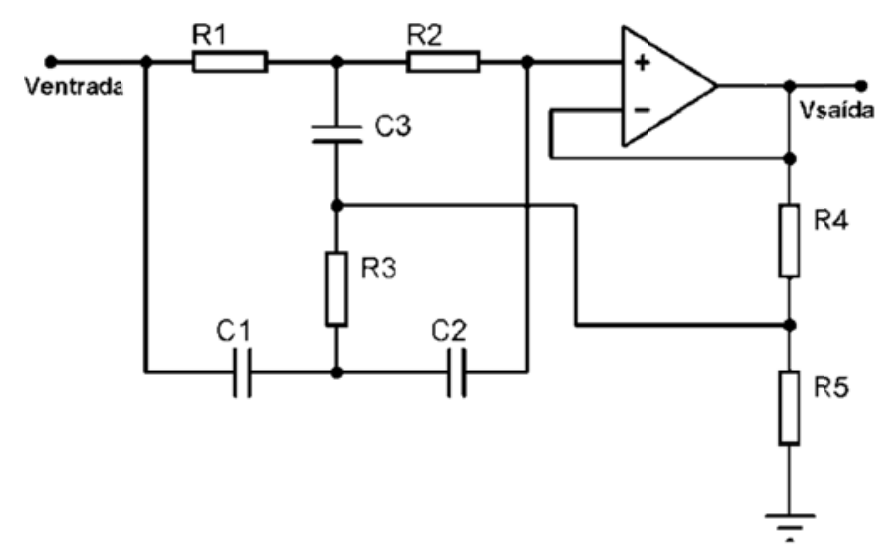

Figura 3.4 - Esquema do filtro notch duplo T rejeita-faixas. 
Adotando para o circuito em questão valores de $R=R_{1}=R_{2}=2 R_{3}$ e $C=C_{1}=C_{2}=\frac{C_{3}}{2}$, obtêm-se a função de transferência dada pela eq.(3.4) e frequência central dada pela equação 3.5 (SEDRA; SMITH, 2007).

$$
\begin{gathered}
A(s)=\frac{s^{2}+\omega_{o}^{2}}{s^{2}+4 \omega_{o}\left(1-\frac{R_{5}}{R_{4}+R_{5}}\right) s+\omega_{o}^{2}} \\
\omega_{o}=\frac{1}{R C}
\end{gathered}
$$

Dessa forma os valores de seletividade são representados por:

$$
Q=\frac{f_{o}}{f_{h}-f_{l}}=\frac{R_{4}+R_{5}}{4 R_{4}}
$$

Com o valor de $\mathrm{R}=\mathrm{R}_{1}=120,57 \mathrm{k} \Omega$ e $\mathrm{R}_{3}=60,28 \mathrm{k} \Omega$, calculou-se a capacitância $\mathrm{C}_{1}=22 \mathrm{nF}$ e $\mathrm{C}_{3}=44 \mathrm{nF}$. Para uma seletividade de $\mathrm{Q}=5,5$, projetou-se os valores de $\mathrm{R}_{4}=4,7 \mathrm{k} \Omega$ e $\mathrm{R}_{5}=100 \mathrm{k} \Omega$. Como os valores de resistores não são encontrados em nenhuma série comercial, foi escolhido o valor de $R_{1}=124 \mathrm{k} \Omega$ e o valor de $\mathrm{R}_{3}$ foi obtido através de uma associação paralela de dois resistores de $124 \mathrm{k} \Omega$, o que equivale a uma resistência elétrica de $62 \mathrm{k} \Omega$.

O projeto do filtro ativo Butterworth passa-baixas de $4^{\mathrm{a}}$ ordem com frequência de corte $100 \mathrm{~Hz}$ tem como esquema o circuito da figura 3.5.

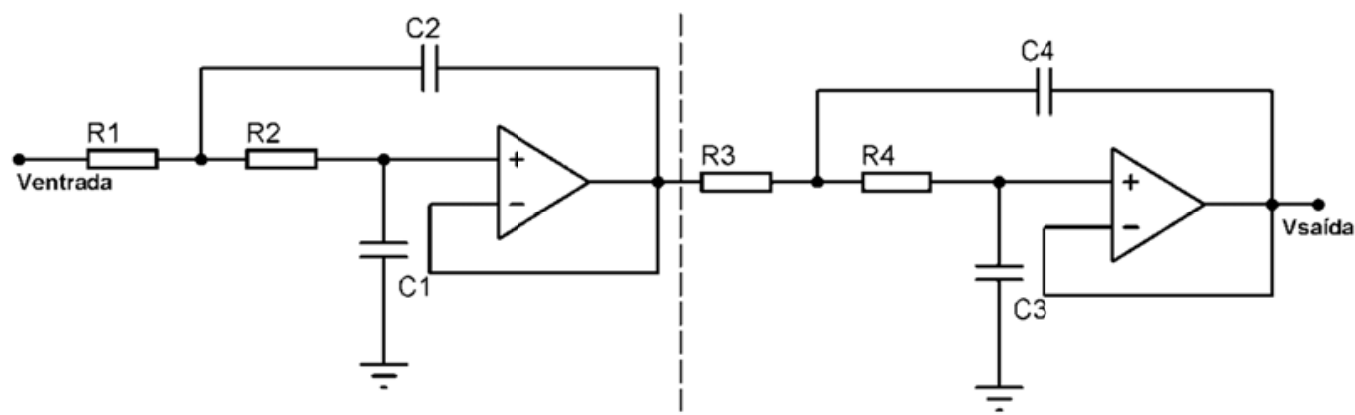

Figura 3.5 - Esquema do filtro Butterworth passa-baixas de $4^{\mathrm{a}}$ ordem. 
Embora a largura de banda do ECG contemple sinais até $250 \mathrm{~Hz}$, Webster (1998) demonstra que uma largura de banda de $100 \mathrm{~Hz}$ já é suficiente para análises e diagnósticos de ECG. O filtro de $4^{\mathrm{a}}$ ordem foi obtido através do cascateamento de dois filtros passa-baixas de $2^{\mathrm{a}}$ ordem. Dado que a função de transferência geral de filtros passa-baixas é representada pela eq.(3.7).

$$
A(s)=\frac{A_{o}}{\prod_{i}\left(1+a_{i} s+b_{i} s^{2}\right)}
$$

Para filtros de $4^{\mathrm{a}}$ ordem com topologia Sallen-Key e ganho $A_{o}=1$, a form.(3.8) apresenta a função de transferência simplificada.

$$
A(s)=\frac{1}{1+\omega_{c} C_{1}\left(R_{1}+R_{2}\right) s+\omega_{c}^{2} R_{1} R_{2} C_{1} C_{2} s^{2}} \cdot \frac{1}{1+\omega_{c} C_{3}\left(R_{3}+R_{4}\right) s+\omega_{c}^{2} R_{3} R_{4} C_{3} C_{4} s^{2}}
$$

A partir da equação acima, encontram-se os valores relativos aos coeficientes $a_{1}=\omega_{c} C_{1}\left(R_{1}+R_{2}\right), b_{1}=\omega_{c}^{2} R_{1} R_{2} C_{1} C_{2}, a_{2}=\omega_{c} C_{3}\left(R_{3}+R_{4}\right)$ e $b_{2}=\omega_{c}^{2} R_{3} R_{4} C_{3} C_{4}$. Para o filtro do tipo Butterworth de $4^{\text {a }}$ Ordem os coeficientes são tabelados de acordo com: $a_{1}=1,8478, b_{1}=1, a_{2}=0,7654$ e $b_{2}=1$ (TEXAS, 2002). Dessa forma para o projeto desse filtro passa-baixas com frequência de corte $100 \mathrm{~Hz}$, utilizou-se resistência fixa, tal que $\mathrm{R}=\mathrm{R}_{1}=\mathrm{R}_{2}=\mathrm{R}_{3}=\mathrm{R}_{4}=39 \mathrm{k} \Omega$, de forma a encontrar os valores de $\mathrm{C}_{1}=$ $37,7 \mathrm{nF}, \mathrm{C}_{2}=41,9 \mathrm{nF}, \mathrm{C}_{3}=15,6 \mathrm{nF}$ e $\mathrm{C}_{4}=106,6 \mathrm{nF}$. Para as etapas posteriores de simulação e prototipagem desse circuito foram utilizados os valores comerciais de $\mathrm{R}$ $=39 \mathrm{k} \Omega, \mathrm{C}_{1}=39 \mathrm{nF}, \mathrm{C}_{2}=46 \mathrm{nF}, \mathrm{C}_{3}=12 \mathrm{nF}$ e $\mathrm{C}_{4}=100 \mathrm{nF}$.

Para a amplificação do sinal foram utilizados dois estágios de amplificadores não inversores conforme a figura 3.6. O primeiro de ganho variável entre 1 e 1000 e o segundo de ganho fixo igual a 10.

Para o primeiro estágio foram calculados os valores de $\mathrm{R}_{1}, \mathrm{R}_{2}$ e $\mathrm{R}_{\text {POT1 }}$ de acordo com a equação 3.9. Com isso foram obtidos os valores para A entre 1 e 1000, através da escolha de $\mathrm{R}_{1}=100 \Omega, \mathrm{R}_{2}=100 \Omega$ e $\mathrm{R}_{\mathrm{POT} 1}=100 \mathrm{k} \Omega$. 


$$
A=1+\frac{R_{1}+R_{P O T 1}}{R_{2}}
$$

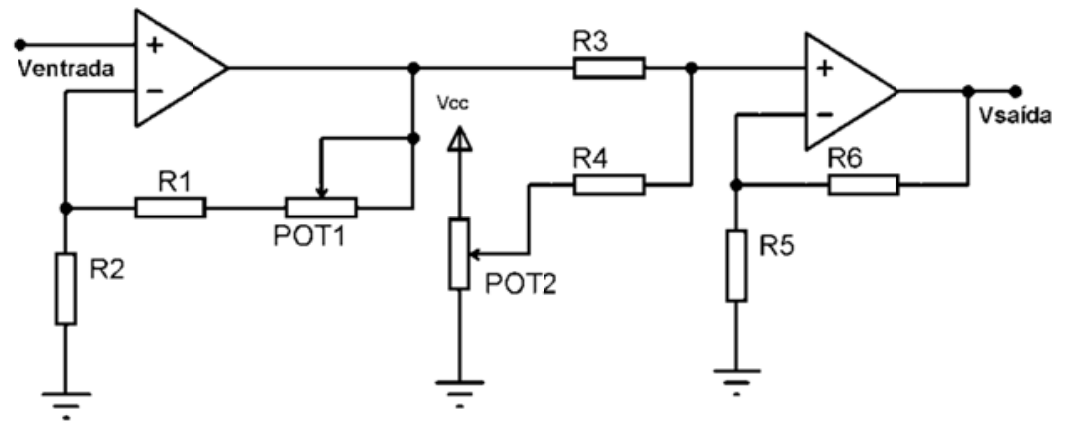

Figura 3.6 - Esquema dos estágios amplificadores não inversores e somadores.

No segundo estágio, foi projetado um amplificador com ganho constante de 10 através da seleção de $\mathrm{R}_{5}=1 \mathrm{k} \Omega$ e $\mathrm{R}_{6}=9,01 \mathrm{k} \Omega$. Além de amplificar esse estágio possibilitou a inserção de um offset no sinal através de um circuito somador. Esse circuito foi projetado para ser utilizado em conversores analógico-digital unipolares.

$$
V_{\text {saida }}=\left[\frac{R_{4}}{R_{3}+R_{4}}\left(1+\frac{R_{1}+R_{P O T 1}}{R_{2}}\right) V_{\text {entrada }}+\frac{R_{3}}{R_{3}+R_{4}} V_{\text {offset }}\right]\left(1+\frac{R_{6}}{R_{5}}\right)
$$

$\mathrm{O}$ circuito somador não inversor foi implementado através da escolha de $\mathrm{R}_{3}=$ $\mathrm{R}_{4}=1 \mathrm{k} \Omega$ e $\mathrm{R}_{\text {РОT2 }}=1 \mathrm{k} \Omega$ de forma a obter uma resposta de tensão dada pela form.(3.10). Sendo que $V_{\text {offset }}$ é uma tensão entre $V_{c c}$ e o terra (referência do circuito), que é ajustada através de um divisor de tensão configurada com o potenciômetro $\mathrm{R}_{\mathrm{POT} 2}$.

Para os filtros passa-altas de $2^{\mathrm{a}}$ ordem tipo Butterworth e rejeita-faixas notch duplo T foram utilizados os circuitos integrados (CI) LF353 (Wide Bandwidth Dual JFET Input Operational Amplifier). Os amplificadores não inversores e somadores, além do filtro passa-baixas de $4^{\mathrm{a}}$ ordem tipo Butterworth foram desenvolvidos utilizando o CI LF347 (Wide Bandwidth Quad JFET Input Operational Amplifiers). Os componentes passivos projetados foram prototipados a partir de resistores de precisão de filme vítreo metalizado, de filme metálico e de montagem de superfície, 
além de capacitores cerâmicos. Todos esses circuitos foram condicionados em uma caixa metálica para proteção contra interferência eletromagnética.

\subsection{Inclinômetros}

Para realizar a medida de ADM dos membros superiores, em particular nesse trabalho, o ângulo formado pelos movimentos de flexão e extensão do cotovelo, utilizou-se um sensor de inclinação. Os possíveis dispositivos sensores que são capazes de medir esse parâmetro são: acelerômetros, através de medidas de aceleração estática (gravidade); giroscópios, pela medida de velocidade angular; encoders, por medida optoeletrônica; e extensômetros, por medidas de deformação. Todos esses sensores medem indiretamente o ângulo de inclinação, para tanto devese realizar uma caracterização específica para que eles correlacionem suas grandezas de saída com a medida de inclinação desejada.

Alguns requisitos foram fundamentais para definir o tipo de sensor a ser utilizado (SOARES et al., 2010). Dentre eles: a facilidade da montagem mecânica do aparato de medida, a sensibilidade do sensor, o circuito para condicionamento do sensor e a facilidade de medida. A partir desses requisitos foi constatado que os acelerômetros integrados atendem às necessidades de um eletrogoniômetro devido seu tamanho reduzido, facilidade de confecção do aparato, além da sua alta sensibilidade e resposta através de grandezas analógicas (tensão) ou digitais (duty cycle).

Os dispositivos acelerômetros são Micro-Eletro-Mecânicos (MEMS). Esses sensores podem ser: capacitivos, piezoelétricos, piezoresistivos, magnéticos ou térmicos. Fabricantes como a Analog Devices, Freescale Semiconductor e a Honeywell desenvolvem esses dispositivos utilizando, em larga medida, tecnologias capacitivas e piezoelétricas.

Fugindo à regra, empresas como a Memsic utilizam tecnologias a partir de sensores térmicos. Esses acelerômetros apresentam uma massa sísmica suspensa por uma viga em uma cápsula preenchida com um gás condutor térmico. $\mathrm{O}$ 
funcionamento do acelerômetro se dá através da transferência de calor a termopilhas alocadas em cada eixo do dispositivo, como demonstrado na figura 3.7.

O acelerômetro MX2125 (Memsic 2125 Dual Axis Acellerometer) (ANEXO D) é um acelerômetro térmico a gás. Nesse acelerômetro a massa sísmica é o próprio gás, que se desloca dentro da cápsula conforme a aceleração. Esse sensor é capaz de medir faixas de aceleração de $\pm 3 \mathrm{~g}$ em dois eixos. Sua largura de banda é de $17 \mathrm{~Hz}$ com sensibilidade de $10^{-3} \mathrm{~g}$.

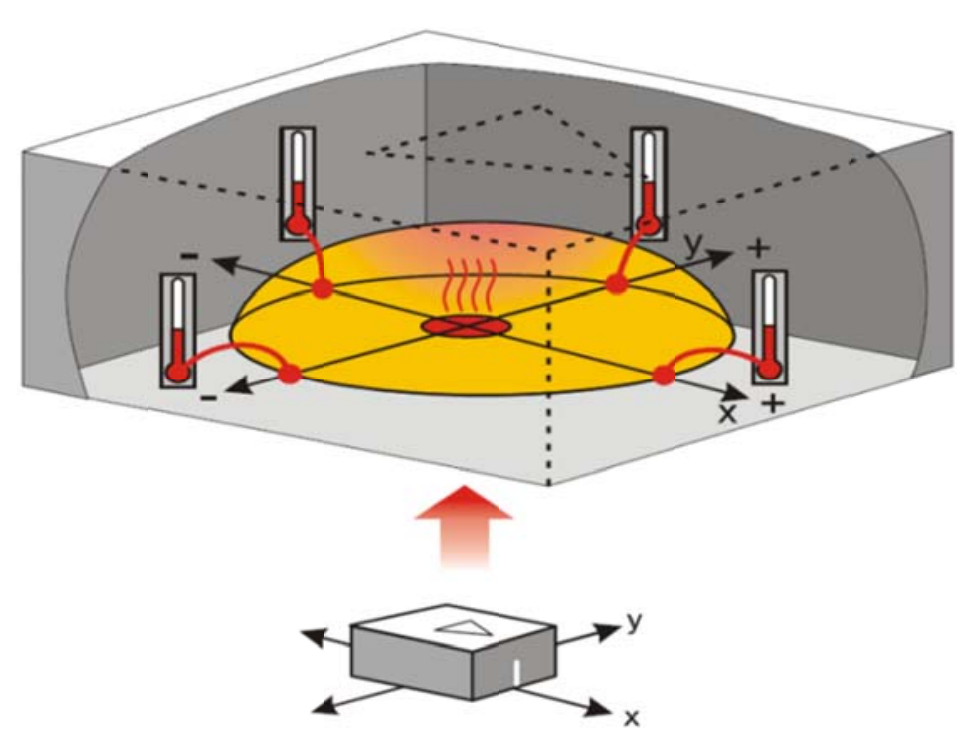

Figura 3.7 - Esquema de funcionamento do acelerômetro a gás (MEMSIC, 2011).

Para realizar a medida do ângulo da ADM foram empregados dois acelerômetros MX2125. Através da medida da aceleração estática, que ocorre devido ao deslocamento da massa sísmica do sensor conforme a orientação da força gravitacional foi possível medir a inclinação em relação aos eixos x e y de cada sensor. Os acelerômetros apresentados na figura 3.8 por "Sensor 1" e "Sensor 2" apresentam os valores da aceleração estática devido à gravidade. Em condição de repouso são mensuráveis os módulos de $G_{\mathrm{x}}$ e $G_{\mathrm{y}}$, que guardam a seguinte relação.

$$
\begin{aligned}
& G_{x}=G \cdot \operatorname{sen} \theta \\
& G_{y}=G \cdot \cos \theta
\end{aligned}
$$




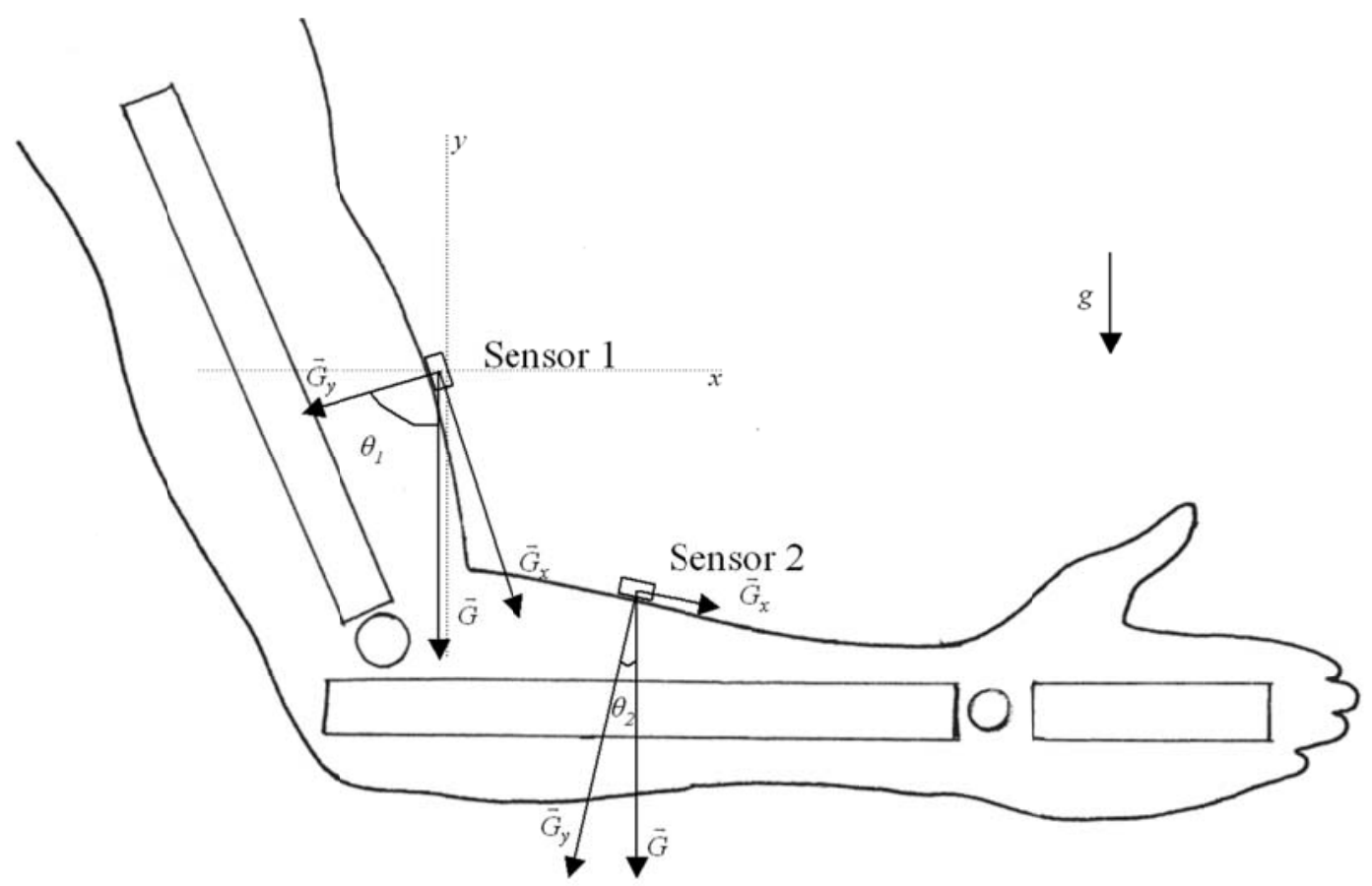

Figura 3.8 - Medida da inclinação das articulações utilizando acelerômetros.

Para encontrar o valor de amplitude do movimento, partiu-se das equações eq.(3.11) e eq.(3.12) e foram obtidos, para cada sensor, os valores da inclinação respectivos ao braço $\left(\theta_{1}\right)$ e antebraço $\left(\theta_{2}\right)$ de acordo com a equação 3.13 .

$$
\theta_{1}=\operatorname{arctg} \frac{G_{x_{1}}}{G_{y_{1}}} \text { e } \theta_{2}=\operatorname{arctg} \frac{G_{x_{2}}}{G_{y_{2}}}
$$

$\mathrm{O}$ valor da amplitude de movimento corresponde à diferença de ângulo entre os sensores, tal como representado na eq.(3.14). Esse valor necessariamente ficará, como visto no subcapítulo 2.6.1, entre $20^{\circ}$ e $180^{\circ}$. Posto que esses são os valores típicos de extensão e flexão do cotovelo.

$$
A D M=\theta_{1}-\theta_{2}
$$




\subsection{Sistemas embarcados microcontrolados}

Para realizar a amostragem dos sinais dos sensores, seu processamento e transmitir a informação para um computador utilizando o protocolo IEEE1451 foram escolhidos microcontroladores da família AVR, da Atmel Semiconductor.

Dentre os possíveis dispositivos de sistemas embarcados, entre eles DSPs (Processador Digital de Sinais), FPGAs (Field-Programmable Gate Array), CPLDs (Complex Programmable Logic Device), ou microcontroladores, optou-se por esses últimos devido a seu alto desempenho, facilidade de aprendizado, baixo consumo de energia e baixo custo.

Os microcontroladores são circuitos digitais que possuem microprocessadores adicionados de memórias e periféricos. Esses dispositivos também são conhecidos como "computadores em um único chip", pois possuem a arquitetura de um computador totalmente embarcada em uma única pastilha. Em específico, os microcontroladores da família AVR possuem 8 bits, tecnologia CMOS (Complementary Metal-Oxide-Semiconductor), arquitetura RISC (Reduced Instruction Set Computing) com 130 instruções e arquitetura Harvard com barramentos distintos para acesso à memória de programa e para acesso à memória de dados, o que permite ao microcontrolador ser altamente pipeline.

Outro fator importante a ser salientado é a disponibilidade de ferramentas gratuitas oferecidas pela Atmel Semiconductor. Entre elas estão o ambiente integrado de desenvolvimento AVRStudio 4.0 e o compilador WinAVR que fornece uma versão do GCC (compilador gratuito da linguagem ANSI C) para a arquitetura AVR.

Nesse contexto, decidiu-se por utilizar dois microcontroladores: o AT90USB162 para o desenvolvimento do STIM e o ATmega8535 para o desenvolvimento do WTIM. O primeiro microcontrolador permitiu a construção de um eletrogoniômetro, pois possui uma interface USB 2.0 Full-speed Device integrada ao microprocessador. Para a leitura dos sensores, o AT90USB162 possui dois contadores de tempo (timer/counter) com precisão de 8 bits.

O segundo microcontrolador atendeu aos requisitos para a construção de um sistema de aquisição de biopotenciais, pois opera com 16 MIPS (Milhões de 
instruções por segundo) a uma frequência de clock de $16 \mathrm{MHz}$, além disso, conta com 8 canais de conversor analógico-digital com precisão de 10 bits e taxa de amostragem de 1000 amostras/segundo ${ }^{10}$, atendendo a condição de Nyquist ${ }^{11}$. O ATmega8535 também possui uma interface $U A R T$, que permite a comunicação com o módulo Wifly $R N-131$, que será discutido posteriormente.

\subsubsection{Interface serial USB}

A interface de comunicação $U S B$ foi desenvolvida com o intuito de permitir que periféricos se conectem e desconectam de dispositivos sem a necessidade de reiniciar o equipamento. Dentre suas características estão: configuração automática, facilidade de conexão, conexão a quente (hot plugged \& swapping) e alimentação de periféricos pela própria interface (AXELSON, 2001).

Esse padrão de comunicação foi proposto pela Intel Corporation em conjunto com outros fabricantes. Na versão 2.0 é possível obter uma taxa de transmissão de até 480Mbps. Outra peculiaridade dessa interface é a possibilidade de ligação através da topologia em árvore, a possibilitar que uma mesma porta acesse 127 periféricos diferentes.

A funcionalidade de um dispositivo USB é definida por sua classe. Essa informação fica armazenada no próprio equipamento e é transmitida ao computador através de três bytes: Base Class, SubClass e Protocol. Dentro das possíveis classes que um periférico USB pode assumir estão: Mass Storage (para dispositivos de memória), Human Interface Device (HID) (para teclados, mouses, entre outros) e Communication Device Class $(C D C)$ (conexão serial, vídeo, áudio e controlador sem fio).

A vantagem de utilizar o microcontrolador AT90USB162 está na possibilidade de utilizar $A P I S$ desenvolvidas que disponibilizam uma implementação das classes Mass Storage, $C D C$ e $H I D$. Dessa forma a transmissão de dados através

\footnotetext{
${ }^{10}$ Valor caracterizado a partir de um programa teste.

11 De acordo com o Teorema de Nyquist para amostrar um sinal sem que ocorra rebatimento é necessária uma taxa de amostragem de no mínimo duas vezes o valor da frequência máxima alcançada pelo sinal analógico.
} 
da USB para o desenvolvimento de um STIM foi obtida através da classe de funcionamento $C D C$, o que garantiu a interação entre esse nó sensor e o $N C A P$.

\subsubsection{Interfaces sem fio Wifi}

O padrão de comunicação para redes locais IEEE802.11, também conhecido como Ethernet sem fio, foi proposto em 1995 com o objetivo de acrescentar uma nova camada física ao modelo ISO/OSI. Seu objetivo era estabelecer um padrão para redes locais sem fio ( $W L A N s$ ) com taxas de transferência de até $2 M b p s$ com camada física tanto por rádio frequência como por infravermelho. Em 1999, o IEEE publicou duas extensões ao padrão, conhecidas por $802.11 \mathrm{a}$ e $802.11 \mathrm{~b}$, que permitiam taxas de transferência de até 54Mbps e $11 M b p s$ respectivamente, usando exclusivamente rádio frequência (OLIVEIRA, 2005).

Detalhadamente, o 802.11a utiliza um esquema especial de multiplexação para atingir altas taxas de comunicação, o que tornou impossível à comunicação entre dispositivos 802.11a e 802.11b. A vantagem do padrão 802.11a está no uso em ambientes externos, pois a sua frequência de operação de $5 \mathrm{GHz}$ reduz consideravelmente a susceptibilidade à interferências.

O 802.11b opera na frequência de $2,4 \mathrm{GHz}$ conhecida como Industrial, Scientific and Medical (ISM). Esta faixa de frequência é aberta para uso em um grande número de países, o que significa que cada dispositivo operando nesta faixa não necessita ser licenciado individualmente. Em 2003, foi lançada a extensão $802.11 \mathrm{~g}$, que se popularizou como uma solução substituta ao $802.11 \mathrm{~b}$, além de ser compatível com esta extensão também é com a 802.11a. A sua taxa de transmissão pode ser de 54Mbps ou $11 M b p s$, dependendo da compatibilidade adotada (OLIVEIRA, 2005).

Para a construção de redes sem fio é possível diferenciar dois tipos de arquitetura. Aquelas no quais os elementos de uma rede móvel podem se comunicar diretamente (ponto-a-ponto) ou com o suporte de uma infraestrutura. $\mathrm{O}$ primeiro tipo 
de rede é conhecido como redes $a d-h o c^{12}$. Esse modelo de arquitetura é muito utilizado em rede de sensores. Por outro lado, a arquitetura de infraestrutura utiliza estações base para interconectar os dispositivos com redes remotas.

Para o desenvolvimento do WTIM foi utilizado o módulo embarcado WiFly $G S X$. Esse módulo permitiu a conexão sem fio através do padrão IEEE802.11b/g, transmitindo sinais na frequência de portadora de 2,4GHz. Além disso, esse periférico possui a pilha TCP/IP (Transmission Control Protocol/Internet Protocol) integrada ao processador embarcado, a permitir a recepção e transmissão dos dados na camada de aplicação. Dessa maneira, com uma simples configuração através da $U A R T$ do microcontrolador ATmega8535, foi factível obter uma conexão sem fio Wifi entre o módulo sensor e o módulo de rede. Em outras palavras, entre o WTIM e o NCAP.

\subsection{Desenvolvimento e implementação do IEEE1451.0}

Como dito anteriormente, a norma IEEE1451.0 foi proposta com numeração retroativa. Essa escolha teve como objetivo dar suporte a novas interfaces e padronizar a estrutura interna do protocolo IEEE1451. Esse padrão permite que diferentes fabricantes desenvolvam NCAPs e TIMs de forma interoperável. Ou seja, os propósitos da norma IEEE1451.0 podem ser vistos pelo próprio título: "Padrão para Interface de Transdutores Inteligentes para Sensores e Atuadores - Funções Comuns, Protocolos de Comunicação e Formato do Transducer Electronic Data Sheet (TEDS)",13.

Ou seja, dentre as finalidades estão: definir tipos de dados, a estrutura das mensagens trocadas entre o NCAP e o TIM, os comandos de controle, o formato do $T E D S$, as APIS disponíveis entre os diferentes blocos do modelo, os modos de operação e transferência que podem ser disponibilizados pelo TIM, a descrição das

\footnotetext{
${ }^{12}$ Do latim: "para esta finalidade".

${ }^{13}$ Do original: Standard for a Smart Transducer Interface for Sensors and Actuators - Common Fuctions, Communication Protocols, and Transducer Electronic Data Sheet (TEDS) Format. Tradução livre.
} 
funções que devem ser realizadas por um $T I M$, além das funções e serviços providos ao NCAP através do módulo de comunicação.

Para a integração do IEEE1451.0 ao IEEE1451.2 e IEEE1451.5, faz-se necessário um detalhamento desse padrão. Tal fato é realizado através da especificação de conceitos, modos de operação, comandos e funções (FILHO et al., 2010) (TORRI, 2008).

\subsubsection{TransducerChannel}

Para o IEEE1451.0 o conjunto formado pelos transdutores, os componentes de aquisição e processamento de sinais é chamado de TransducerChannel. Cada TransducerChannel é associado a um número de identificação de 16 bits (de 1 a 65535). Esse valor serve para distinguir os diferentes sensores existentes no mesmo módulo TIM. Além disso, permite endereçar os comandos enviados pelo NCAP.

Através dessa definição, o IEEE1451.0 demonstra a possibilidade de um único TIM conter até 65535 TransducerChannels diferentes. Em outras palavras, até 65535 nós sensores conectados a um único $N C A P$. No caso da arquitetura proposta foram utilizados dois TransducerChannels, um para o WTIM e outro para o STIM.

\subsubsection{Modos de Operação}

O padrão IEEE1451.0 também se ocupa em descrever os modos de operação do TransducerChannel. Entre esses modos estão o de amostragem (sampling) e o de transmissão (transmission modes). Por meio da configuração e seleção desses modos é que se dá a operação dos transdutores.

A amostragem descreve como o TransducerChannel efetua a obtenção de dados, no caso de um sensor, ou como os dados recebidos devem ser utilizados pelo atuador. São cinco os modos de amostragem descritos na norma: 
- Trigger-initiated - $\mathrm{O}$ sensor realiza a aquisição de dados assim que recebe um comando de início, disparado pelo TIM. O atuador, por outro lado, inicia sua funcionalidade a partir desse mesmo comando.

- Free-running without pre-trigger - $\mathrm{Na}$ condição de sensor, o TransducerChannel lerá os dados de um sensor e logo em seguida esses serão descartados. Somente com a ocorrência de um comando de trigger, as leituras passarão a ser armazenadas até que estejam completas para transmissão.

-Free-running with pre-trigger - Nesse modo de operação o sensor efetua e armazena as leituras de forma autônoma e contínua, até que um comando do tipo trigger seja recebido. Esse modo de operação permite detalhar duas condições de funcionamento descritas abaixo.

- Free-running with pre-trigger without buffer enabled - Quando um conjunto de dados preenche por completo um buffer, as próximas leituras são descartadas até que o TransducerChannel receba um comando de leitura ou de trigger.

- Free-running with pre-trigger and buffer enabled - $\mathrm{O}$ TransducerChannel permanece continuamente armazenando as informações em buffers de memória do sensor. Caso não existam mais buffers vazios, as próximas leituras são descartadas até que um buffer seja disponibilizado mediante sua leitura.

- Continuos sampling - As amostras são armazenadas em um dos buffers do sensor somente com o recebimento de um evento inicial. A operação é semelhante ao modo "free-running without pre-trigger", sendo que a diferença está ligada ao fato do TransducerChannel não parar de realizar aquisições, mesmo com seu buffer cheio.

- Immediate operation - Após o recebimento de uma leitura de dados, o TransducerChannel efetua a aquisição dos dados dos sensores e responde ao requisito do $N C A P$. No caso de um atuador, os dados de controle recebidos juntos com o comando de escrita são imediatamente utilizados na atuação. 
Acerca do modo de transmissão, esse determina como é feita a transferência das informações amostradas ou enviadas entre o NCAP e o TIM. Os modos que cada TransducerChannel podem operar são atribuídos no TEDS de cada transdutor e são descritos por:

- Only when commanded - O TransducerChannel só envia o conjunto de dados em resposta a um comando de leitura.

- Streaming when a buffer is full - O transdutor transmite os dados, assim que o conjunto de informação requisitado tenha sido adquirido por completo.

- Streaming at a fixed interval - O TransducerChannel transmite os dados com uma periodicidade fixa. O número de amostras é determinado a partir da taxa de amostragem e do intervalo de transmissão.

Para o desenvolvimento dos nós sensores biomédicos foram utilizados o modo de amostragem Immediate operation e o modo de transmissão Only when commanded. Através desses modos de operação permitiu-se ao operador, no caso um médico, requisitar as informações somente no momento em que o paciente estiver com os sensores devidamente colocados. Além disso, esses modos eliminam qualquer tipo de operação redundante ou que demande transmissão de dados desnecessários pela rede, especialmente, quando o operador estiver recebendo remotamente a aquisição de dados.

\subsubsection{Especificação do TEDS}

Os valores do TEDS também são padronizados pelo IEEE1451.0. Como dito anteriormente, a principal característica do padrão IEEE1451 é a possibilidade de armazenar um conjunto de informações nos nós transdutores com o intuito de descrever um TIM ou um TransducerChannel. Essas informações permitem identificar e disponibilizar os dados dos sensores/atuadores de forma única e universal. Alguns TEDS podem ser armazenados em lugares distintos ao TIM, nesse 
caso são chamados TEDS virtuais. Entretanto, tais $T E D S$ não são tão utilizados como os integrados no próprio sensor.

Há um formato padrão para a construção de TEDS. Sua estrutura, apresentada na tabela 3.2, é formada por três campos: o comprimento, os dados e o checksum. O TEDS length (comprimento), corresponde a um inteiro sem sinal de 32 bits (quatro octetos). Já o Data Block (dados), segundo campo, apresenta o conteúdo informativo do TEDS. A estrutura e o tamanho são específicos para cada tipo de TEDS, a permitir que a informação seja armazenada em forma de octetos ou em forma de texto. Por último, o checksum corresponde ao complemento da soma de todos os octetos anteriores, como descrito na eq.(3.15). Esse valor serve para garantir a integridade do dado transmitido.

Tabela 3.2 - Formato do TEDS.

\begin{tabular}{llll}
\hline Campo & Descrição & Tipo & Número de Octetos \\
\hline-- & Comprimento & UInt32 & 4 \\
1 até N & Dados & Variável & Variável \\
-- & Checksum & UInt16 & 2 \\
\hline
\end{tabular}

Checksum $=0 x F F F-\sum_{i=1}^{\text {Totaloctects }-2} \operatorname{OctetosTEDS}(i)$

Os TEDS utilizam no bloco de dados uma estrutura TLV (Type/Length/Value) formado por três campos com diferentes funcionalidades. Como apresentado na figura 3.9

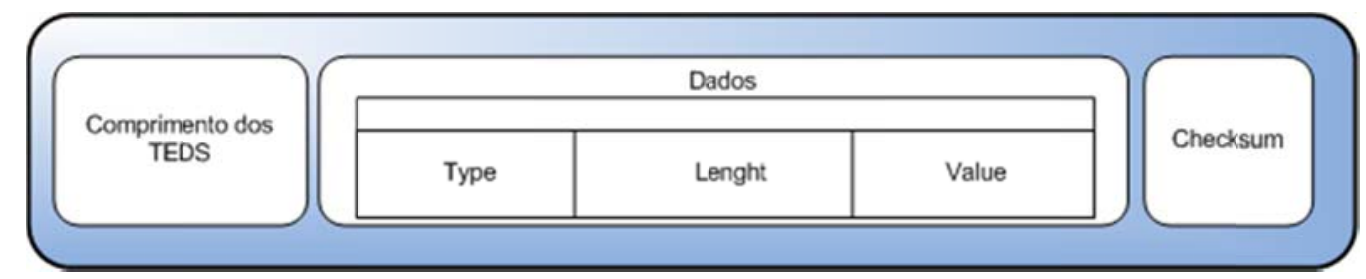

Figura 3.9 - Formato do TEDS. 
O primeiro campo "Type" possui um octeto e identifica o início e tipo de um $T L V$. Já o campo "Lenght" representa o número de octetos que constituem o campo "Value". Por fim o campo "Value" contém as informações armazenadas nos TEDS. Para a construção dos TEDS a primeira TLV é composta pelo TEDS identification header (TEDSID), e tem o objetivo de identificar o TEDS descrito. Sua estrutura pode ser vista na tabela 3.3 .

Tabela 3.3 - TEDS identification header.

\begin{tabular}{ll}
\hline Campo & Descrição \\
\hline Type & Identifica a $T L V$. \\
\hline Length & Indica que o campo Value possui quatro octetos. \\
\hline & Indica a norma que está sendo representada pelo TEDS. Para \\
Family & $T E D S$ da norma IEEE1451.0 esse campo é indicado com 0. \\
& Para PHY TEDS, esse campo é indicado com o número da \\
& norma da camada física do sensor. \\
\hline Class & Identifica qual o tipo do TEDS. \\
\hline Version & Campo que identifica a versão dos TEDS. \\
\hline \multirow{2}{*}{ Tuple Length } & Indica o número de octetos no campo length de todas as \\
& $T L V S$. \\
\hline
\end{tabular}

O processo de descrição do TEDS consiste em seguir os formatos definidos pela norma e atribuir valores para cada um dos campos conforme o TIM que está sendo descrito. Para tanto, na norma IEEE1451, é necessário configurar quatro TEDS obrigatórios para todos os TIMs. São eles: Meta TEDS, TransducerChannel TEDS, User's Transducer Name TEDS e PHY TEDS. Além dos TEDS obrigatórios, a norma possui outros opcionais para a configuração, como apresentados na figura 3.10.

Sobre os TEDS obrigatórios, podem-se listar as seguintes características:

- Meta TEDS - Realiza a identificação do TIM e as informações sobre os canais de transdutores, bem como seu acesso.

- TransducerChannel TEDS - Descreve as informações detalhadas sobre cada canal e as características do transdutor. É nesse TEDS que se armazena as informações sobre unidades físicas, incertezas, temporização, faixa de operação e características de I/O (input/output) digital. 
-User's Transducer Name TEDS - Armazena o nome de reconhecimento do TIM ou do TransducerChannel.

- PHY TEDS - Armazena as informações sobre os métodos de acesso ao meio físico específico para a comunicação entre o TIM e o NCAP.

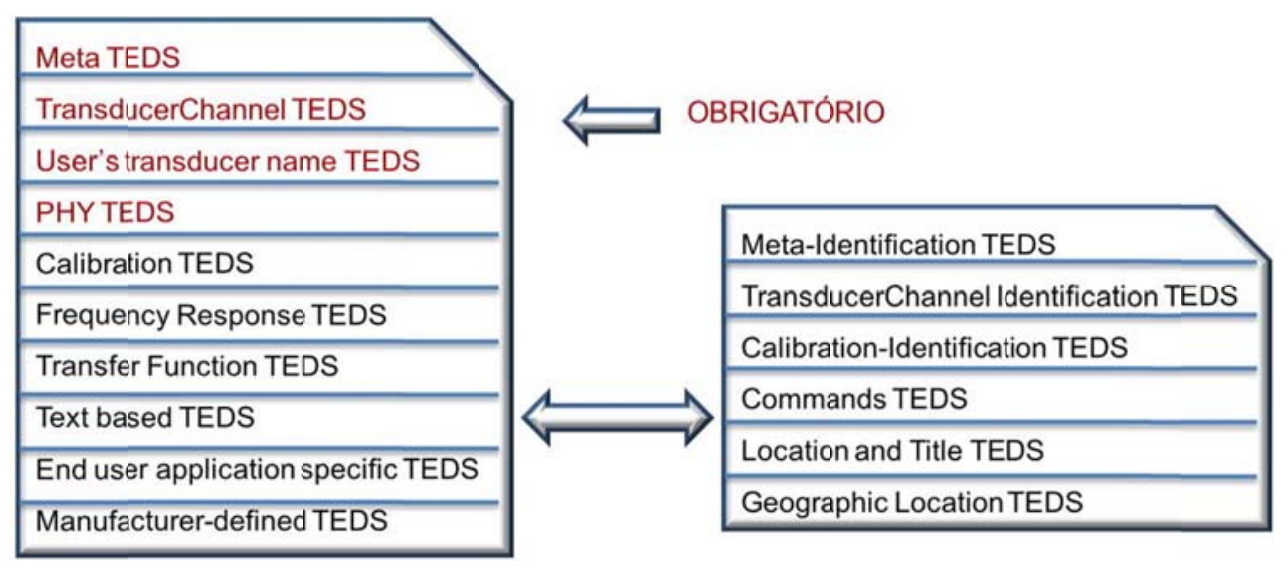

Figura 3.10 - TEDS do IEEE1451.0 (SONG; LEE, 2008).

Embora de grande utilidade, nesse projeto não foi considerado a inclusão dos TEDS não obrigatórios. Ou seja, os seguintes TEDS: Calibration TEDS, Frequency Response TEDS, Transfer Function TEDS, Text-based TEDS, Command TEDS, Identification TEDS, Geographic Location TEDS, Unit Extension TEDS, End User Application Specific TEDS e Manufacturer Defined TEDS.

Do anexo E é possível verificar todos os campos que compõem o Meta TEDS. Contudo somente os campos apresentados nas tabelas 3.4 e 3.5 são obrigatórios. Sendo que a tabela 3.4 representa a configuração do Meta TEDS para o STIM do projeto e a tabela 3.5 representa a configuração do Meta TEDS para o WTIM. Nessas tabelas é possível verificar o nome de cada campo do Meta TEDS, a descrição do funcionamento, o comprimento, o tipo do campo e o conteúdo declarado para a informações do STIM e do WTIM, respectivamente. Os octetos atribuídos estão representados em hexadecimal, sistema de numeração posicional que representa os números na base 16 . 
Tabela 3.4 - Meta TEDS do STIM.

\begin{tabular}{|c|c|c|c|c|c|}
\hline \multicolumn{6}{|c|}{ Meta TEDS - STIM } \\
\hline 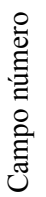 & Nome & Descrição & $\begin{array}{c}\text { Comprimento } \\
\text { do Campo } \\
\text { (Bytes) }\end{array}$ & 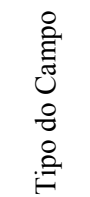 & Conteúdo do Campo \\
\hline & & $\begin{array}{l}\text { Informações relacionadas à Estrutura de } \\
\text { Dados }\end{array}$ & & & \\
\hline- & Lenght & Número de octetos para descrever o Meta TEDS. & 4 & U32 & $\begin{array}{l}\text { 0x00 0x00 0x00 } \\
0 \times 26\end{array}$ \\
\hline 3 & TEDSID & Identificação do TEDS. & 4 & U32 & $\begin{array}{l}\text { 0x00 0x01 0x01 } \\
\text { 0x01 }\end{array}$ \\
\hline \multirow[t]{2}{*}{4} & $U U I D$ & Identificador único do TIM. & 10 & UUID & $\begin{array}{l}\text { 0x00 0x00 0x00 } \\
\text { 0x00 0x00 0x00 } \\
\text { 0x00 0x00 0x00 } \\
\text { 0x00 }\end{array}$ \\
\hline & & Informações de Tempo & & & \\
\hline 10 & OHoldOff & Tempo para resposta de operação de falha. & 4 & F32 & $\begin{array}{l}\text { 0x00 0x00 0x00 } \\
0 \times 00\end{array}$ \\
\hline \multirow[t]{2}{*}{12} & TestTime & Autoteste do módulo TIM. & 4 & F32 & $\begin{array}{l}\text { 0x00 0x00 0x00 } \\
0 \times 00\end{array}$ \\
\hline & & Número de TransducerChannels implementad & & & \\
\hline 13 & MaxChan & Quantidade de canais implementados no TIM. & 2 & U16 & $0 \times 000 \times 01$ \\
\hline- & Checksum & Soma dos Octetos dentro da tabela Meta TEDS. & 2 & U8 & 0xFF 0xD5 \\
\hline
\end{tabular}

O valor Lenght descreve o número de octetos do Meta TEDS. Para esse valor são considerados a identificação de $T L V$ (12 octetos), somada aos octetos do Meta TEDS, no qual se inclui o Checksum. Os valores restantes são indicados nos itens seguintes:

-TEDSID: os valores configurados são, respectivamente, 0x00, 0x01, 0x01 e 0x01. Esses valores configuram o TEDS para a norma IEEE1451.0, com classe Meta TEDS e para o formato atualizado da versão IEEE1451.0.

- UUID: esse campo é formado pela composição de quatro subcampos (localização, fabricante, ano e tempo). Contudo optou-se em não configurar os valores do Universal Unique Identifier para o STIM ou para o WTIM.

- OHoldOff: o valor de tempo de resposta a falha foi colocado em zero, pois essa função não foi utilizada no desenvolvimento dos nós TIMs.

- TestTime: o valor de autoteste também foi configurado para zero, devido a impossibilidade dos nós sensores realizarem esse procedimento. 
- MaxChan: devido a utilização de um único TransducerChannel para o STIM e para o WTIM, esse campo foi preenchido com o valor 0x01.

- Checksum: valor foi calculado a partir da equação 3.15.

Tabela 3.5 - Meta TEDS do WTIM.

\begin{tabular}{|c|c|c|c|c|c|}
\hline \multicolumn{6}{|c|}{ Meta TEDS - WTIM } \\
\hline 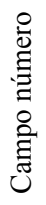 & Nome & Descrição & $\begin{array}{c}\text { Comprimento } \\
\text { do Campo } \\
\text { (Bytes) }\end{array}$ & 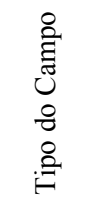 & Conteúdo do Campo \\
\hline \multicolumn{6}{|c|}{ Informações relacionadas à Estrutura de Dados } \\
\hline- & Lenght & $\begin{array}{l}\text { Número de octetos para descrever o Meta } \\
\text { TEDS. }\end{array}$ & 4 & $\mathrm{U} 32$ & $\begin{array}{l}0 \times 000 \times 000 \times 00 \\
0 \times 26\end{array}$ \\
\hline 3 & $T E D S I D$ & Identificação do $T E D S$. & 4 & $\mathrm{U} 32$ & $\begin{array}{l}\text { 0x00 0x01 0x01 } \\
0 \times 01\end{array}$ \\
\hline 4 & $U U I D$ & Identificador único do TIM. & 10 & UUID & $\begin{array}{l}\text { 0x00 0x00 0x00 } \\
\text { 0x00 0x00 0x00 } \\
\text { 0x00 0x00 0x00 } \\
\text { 0x00 }\end{array}$ \\
\hline \multicolumn{6}{|c|}{ Informações de Tempo } \\
\hline 10 & OHoldOff & Tempo para resposta de operação de falha. & 4 & F32 & $\begin{array}{l}\text { 0x00 0x00 0x00 } \\
0 \times 00\end{array}$ \\
\hline 12 & TestTime & Autoteste do módulo TIM. & 4 & F32 & $\begin{array}{l}\text { 0x00 0x00 0x00 } \\
0 \times 00\end{array}$ \\
\hline \multicolumn{6}{|c|}{ Número de TransducerChannels implementados } \\
\hline 13 & MaxChan & Quantidade de canais implementados no TIM. & 2 & U16 & $0 \times 000 \times 01$ \\
\hline- & Checksum & Soma dos Octetos dentro da tabela Meta TEDS. & 2 & U8 & 0xFF 0xD5 \\
\hline
\end{tabular}

$\mathrm{O}$ anexo $\mathrm{F}$ apresenta as informações relativas aos campos que constituem o TransducerChannel TEDS. A tabela 3.6 representa a configuração desse TEDS para o STIM do projeto e a tabela 3.7 representa a descrição do TEDS para o WTIM.

Da mesma maneira apresentada nas tabelas anteriores, os valores nas tabelas de TransducerChannel TEDS descrevem os nomes de cada campo, seu funcionamento, o comprimento, o tipo dos campos e o conteúdo declarado para a informações do STIM e do WTIM, respectivamente. 
Tabela 3.6 - TransducerChannel TEDS do STIM.

\begin{tabular}{|c|c|c|c|c|c|}
\hline \multicolumn{6}{|c|}{ TransducerChannel TEDS - STIM } \\
\hline 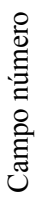 & Nome & Descrição & $\begin{array}{l}\text { Comprimento } \\
\text { do Campo } \\
\text { (Bytes) }\end{array}$ & 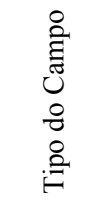 & Conteúdo do Campo \\
\hline & & \multicolumn{4}{|c|}{ Informações relacionadas à Estrutura de Dados } \\
\hline- & Lenght & Número de octetos para descrever o TEDS. & 4 & U32 & $0 \times 000 \times 000 x 000 x 41$ \\
\hline \multirow[t]{2}{*}{3} & TEDSID & Identificação do $T E D S$. & 4 & U32 & 0x00 0x03 0x01 0x01 \\
\hline & & $\begin{array}{l}\text { Informações relativas ao } \\
\text { TransducerChannel }\end{array}$ & & & \\
\hline 10 & CalKey & $\begin{array}{l}\text { Calibração do sensor. Para módulo sem } \\
\text { calibração, atribui-se } 0 .\end{array}$ & 1 & U8 & $0 \times 00$ \\
\hline 11 & ChanType & $\begin{array}{l}\text { Identificação do canal do } \\
\text { TransducerChannel. }\end{array}$ & 1 & U8 & $0 \times 1 E$ \\
\hline 12 & PhyUnits & Descreve a unidade física medida. & 11 & UNITS & $\begin{array}{l}\text { 0x00 0x00 0x00 0x00 } \\
\text { 0x00 0x00 0x00 0x00 } \\
\text { 0x00 0x00 0x00 }\end{array}$ \\
\hline 60 & UnitsExt & Extensão de unidades. & 1 & U8 & $0 \times \mathrm{BB} 0$ \\
\hline 13 & LowLimit & Valor mínimo da unidade a ser medida. & 4 & F32 & 0x00 0x00 0x00 0x00 \\
\hline 14 & HiLimit & Valor máximo da unidade a ser medida. & 4 & F32 & 0x00 0xDC 0x00 0x00 \\
\hline 15 & OError & Incerteza da medida. & 4 & F32 & 0x01 0x00 0x00 0x00 \\
\hline \multirow[t]{2}{*}{16} & SelfTest & $\begin{array}{l}\text { Tempo limite para realizar autoteste. Para } \\
\text { módulo sem autoteste, atribui-se } 0 \text {. }\end{array}$ & 1 & U8 & $0 \times 01$ \\
\hline & & $\begin{array}{l}\text { Informações relativas à conversão de } \\
\text { dados }\end{array}$ & & & \\
\hline \multirow[t]{2}{*}{18} & Sample & $\begin{array}{l}\text { Campo representado por } 3 \text { subcampos: Data } \\
\text { Model, Data Model Lenght e Model } \\
\text { Significant Bits. }\end{array}$ & 9 & & $\begin{array}{l}\text { 0x28 0x01 0x05 0x29 } \\
\text { 0x01 0x02 0x2A 0x01 } \\
\text { 0x0C }\end{array}$ \\
\hline & & Informações de Tempo & & & \\
\hline & $R S e t u p T$ & $\begin{array}{l}\text { Tempo de amostragem mínimo, em } \\
\text { segundos, realizado pelo conversor A/D. }\end{array}$ & 4 & F32 & 0x00 0x00 0x00 0x00 \\
\hline 23 & SPeriod & Configuração para período de amostragem & 4 & F32 & $\begin{array}{l}\text { 0x3D 0xCC 0xCC } \\
0 \times C D\end{array}$ \\
\hline & WarmUpT & $\begin{array}{l}\text { Tempo necessário para o } \\
\text { TransducerChannel entrar em operação. }\end{array}$ & 4 & F32 & $0 \times 400 \times F 00 \times 000 \times 00$ \\
\hline 25 & RDelay $T$ & Atraso máximo para envio de comando. & 4 & F32 & $0 \times 400 \times F 00 \times 000 \times 00$ \\
\hline \multirow[t]{2}{*}{26} & TestTime & Tempo necessário para realizar o autoteste. & 4 & F32 & 0x00 0x00 0x00 0x00 \\
\hline & & Informação do tempo de amostragem & & & \\
\hline 31 & Sampling & Descreve o tipo de amostragem. & 1 & U8 & 0x30 0x01 0x01 \\
\hline & Checksum & Soma dos Octetos dentro da tabela $T E D S$. & 2 & U16 & $0 \times 580 x 48$ \\
\hline
\end{tabular}


Tabela 3.7 - TransducerChannel TEDS do WTIM.

\begin{tabular}{|c|c|c|c|c|c|}
\hline \multicolumn{6}{|c|}{ TransducerChannel TEDS - WTIM } \\
\hline 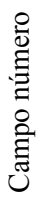 & Nome & Descrição & $\begin{array}{l}\text { Comprimento } \\
\text { do Campo } \\
\text { (Bytes) }\end{array}$ & 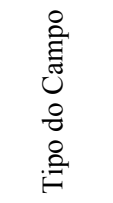 & Conteúdo do Campo \\
\hline & & \multicolumn{4}{|c|}{ Informações relacionadas à Estrutura de Dados } \\
\hline- & Lenght & Número de octetos para descrever o $T E D S$. & 4 & $\mathrm{U} 32$ & $0 x 000 x 000 x 000 x 41$ \\
\hline \multirow[t]{2}{*}{3} & TEDSID & Identificação do TEDS. & 4 & $\mathrm{U} 32$ & 0x00 0x03 0x01 0x01 \\
\hline & & $\begin{array}{l}\text { Informações relativas ao } \\
\text { TransducerChannel }\end{array}$ & & & \\
\hline 10 & CalKey & $\begin{array}{l}\text { Calibração do sensor. Para módulo sem } \\
\text { calibração, atribui-se } 0 .\end{array}$ & 1 & U8 & $0 \times 00$ \\
\hline 11 & ChanType & $\begin{array}{l}\text { Identificação do canal do } \\
\text { TransducerChannel. }\end{array}$ & 1 & U8 & $0 \times 14$ \\
\hline 12 & PhyUnits & Descreve a unidade física medida. & 11 & UNITS & $\begin{array}{l}\text { 0x00 0x00 0x00 0x00 } \\
\text { 0x00 0x00 0x00 0x00 } \\
\text { 0x00 0x00 0x00 }\end{array}$ \\
\hline 60 & UnitsExt & Extensão de unidades. & 1 & U8 & $0 \times 62$ \\
\hline 13 & LowLimit & Valor mínimo da unidade a ser medida. & 4 & F32 & 0x00 0x00 0x00 0x00 \\
\hline 14 & HiLimit & Valor máximo da unidade a ser medida. & 4 & F32 & 0x01 0x2C 0x00 0x00 \\
\hline 15 & OError & Incerteza da medida. & 4 & F32 & 0x02 0x00 0x00 0x00 \\
\hline \multirow[t]{2}{*}{16} & SelfTest & $\begin{array}{l}\text { Tempo limite para realizar autoteste. Para } \\
\text { módulo sem autoteste, atribui-se } 0 \text {. }\end{array}$ & 1 & U8 & $0 \times 01$ \\
\hline & & $\begin{array}{l}\text { Informações relativas à conversão de } \\
\text { dados }\end{array}$ & & & \\
\hline \multirow[t]{2}{*}{18} & Sample & $\begin{array}{l}\text { Campo representado por } 3 \text { subcampos: Data } \\
\text { Model, Data Model Lenght e Model } \\
\text { Significant Bits. }\end{array}$ & 9 & & $\begin{array}{l}\text { 0x28 0x01 0x05 0x29 } \\
\text { 0x01 0x02 0x2A 0x01 } \\
\text { 0x0C }\end{array}$ \\
\hline & & Informações de Tempo & & & \\
\hline 22 & $R S e t u p T$ & $\begin{array}{l}\text { Tempo de amostragem mínimo, em } \\
\text { segundos, realizado pelo conversor A/D. }\end{array}$ & 4 & F32 & 0x00 0x00 0x00 0x00 \\
\hline 23 & SPeriod & Configuração para período de amostragem. & 4 & F32 & $\begin{array}{l}\text { 0x3D 0xCC 0xCC } \\
0 \times C D\end{array}$ \\
\hline 24 & WarmUpT & $\begin{array}{l}\text { Tempo necessário para o } \\
\text { TransducerChannel entrar em operação. }\end{array}$ & 4 & F32 & 0x40 0xF0 0x00 0x00 \\
\hline 25 & RDelayT & Atraso máximo para envio de comando. & 4 & F32 & 0x40 0xF0 0x00 0x00 \\
\hline \multirow[t]{2}{*}{26} & TestTime & Tempo necessário para realizar o autoteste. & 4 & F32 & 0x00 0x00 0x00 0x00 \\
\hline & & Informação do tempo de amostragem & & & \\
\hline 31 & Sampling & Descreve o tipo de amostragem. & 1 & U8 & 0x30 0x01 0x01 \\
\hline & Checksum & Soma dos Octetos dentro da tabela $T E D S$. & 2 & U16 & 0xF9 0x4E \\
\hline
\end{tabular}

Dentre as informações detalhadas por esse TEDS estão: a faixa em que o transdutor opera, as unidades do sensor, os limites inferiores, os limites superiores do 
sensor, a incerteza na medida, além de variáveis de latência de tempo na transmissão de informações. Tais detalhes são descritos a seguir:

- TEDSID: os valores configurados são, respectivamente, 0x00, 0x03, 0x01 e 0x01. Esses valores configuram o TEDS para a norma IEEE1451.0, com classe TransducerChannel TEDS e para o formato atualizado da versão IEEE1451 formado somente por octetos.

- CallKey: como não há possibilidade de calibração com os sensores desenvolvidos, esse campo recebeu valor zero tanto para o STIM como para o WTIM.

- ChanType: a identificação do TransducerChannel do STIM foi realizada com o número 30 (0x1E), enquanto que o WTIM teve numeração de 20 (0x14).

- PhyUnits: nesse campo foram descritas as unidades físicas do sensor de ADM e do sensor de frequência cardíaca. Como os valores de graus e batimento por minuto não estão dentro da lista de unidades físicas do IEEE1451.0, utilizou-se o campo de extensão de unidades do TEDS (TEDS acess code for units extension). Nesse campo a unidade foi marcada em a codificação ASCII (American Standard Code for Information Interchange).

- LowLimit: o valor mínimo tanto para o sensor de ADM como para o sensor de frequência cardíaca foi configurado como zero.

- HighLimit: o valor máximo para o sensor de ADM foi configurado com $220^{\circ}(0 \times 00$ 0xDC 0x00 0x00), enquanto que o sensor de frequência cardíaca foi configurado com 300bpm (0x01 0x2C 0x00 0x00).

- OError: a incerteza do STIM foi configurada em $1^{\circ}$ e a incerteza do WTIM em $2 \mathrm{bpm}$ (valor estimado).

- SelfTest: como não há autoteste, o valor limite para a realização dessa função foi marcado como zero.

- Sample: dos três subcampos obrigatórios (Data model, Data Model Lenght e Model significant bits), foi configurado o primeiro como valor inteiro do 
tipo Long Integer (unsigned), o segundo campo com o comprimento dos octetos e, por último, com a quantidade de bits máxima amostrada pelos sensores (12).

- UpdateT: esse campo expressa o tempo máximo, em segundos, entre um evento de disparo e o envio da primeira amostra do TransducerChannel. Tal campo foi configurado com o valor de 10 segundos para ambos os nós.

- RSetupT: esse campo expressa o tempo máximo entre o disparo de uma requisição de dados até o momento que esse pode ser lido. Como o sensor não demanda esse tempo de latência o valor configurado para esse campo foi zero.

- SPeriod: a configuração para período de amostragem mínimo dos sensores, expresso em segundos, foi de $0,1 \mathrm{~s}$.

-WarmUpt: o tempo necessário para o TransducerChannel entrar em modo de operação foi configurado em 30s (valor estimado).

- RDelayT: o atraso máximo para que um comando do TransducerChannel seja encaminhado foi configurado em $25 \mu$ s (valor estimado).

- TestTime: como não há autoteste nos sensores, o valor foi configurado como zero.

- Sampling: para o modo de amostragem suportado por ambos os módulos TIMs, foi configurado no campo SampMode o valor um no bit menos significativo e no campo SDefault o mesmo valor. Dessa forma ambos os sensores atuam no modo de amostragem Immediate Operation e no modo de transmissão Only when commanded.

- Checksum: valor calculado de acordo com a equação 3.15 .

O último TEDS obrigatório representado pela norma IEEE1451.0 pode ser visto no anexo G. Nesse anexo são apresentados os campos do User's Transducers Name TEDS. A tabela 3.8 apresenta a configuração desse TEDS para o STIM, enquanto que a tabela 3.9 apresenta a descrição desse TEDS para o WTIM. Destacase dessa tabela o campo TCName, que define o nome de cada nó TIM. 
-TEDSID: os valores configurados são, respectivamente: 0x00, 0x0C 0x01 e $0 x 01$. Esses valores configuram o TEDS para a norma IEEE1451.0, com classe User's Transducer Name TEDS e para o formato atualizado da versão IEEE1451 formado somente por octetos.

- Format: para definir o tipo de representação do TCName foi optado pelo formato de texto. Para tanto esse campo foi preenchido com zero.

- TCName: esse campo descreve o nome do STIM e do WTIM. No caso do STIM foi configurado o nome "IEEE1451.2 Accelerometer_Sensor" e no WTIM o nome "IEEE1451.5 ECG_Biopotencial_Sensor". Esse texto foi codificado no formato ASCII.

- Checksum: valor calculado de acordo com a equação 3.15.

Tabela 3.8 - User's Transducers Name TEDS do STIM.

\begin{tabular}{|c|c|c|c|c|c|}
\hline \multicolumn{6}{|c|}{ User's Transducers Name - STIM } \\
\hline 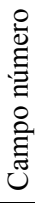 & Nome & Descrição & $\begin{array}{l}\text { Comprimento } \\
\text { do Campo } \\
\text { (Bytes) }\end{array}$ & 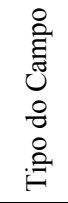 & Conteúdo do Campo \\
\hline & & \multicolumn{4}{|c|}{ Informações relacionadas à Estrutura de Dados } \\
\hline- & Lenght & Número de octetos para descrever o TEDS. & 4 & U32 & 0x00 0x00 0x00 0x26 \\
\hline 3 & TEDSID & Identificação do TEDS. & 4 & U32 & 0x00 0x0C 0x01 0x01 \\
\hline 4 & Format & $\begin{array}{l}\text { Define o formato de representação do } \\
\text { TCName. O TCName descreve o nome do } \\
\text { TIM de acordo com o padrão definido no } \\
\text { formato. }\end{array}$ & 1 & U8 & $0 \times 00$ \\
\hline 5 & TCName & Nome do STIM. & - & - & $\begin{array}{l}\text { 0x49 } 0 \times 450 \times 450 \times 45 \\
\text { 0x31 } 0 \times 34 \text { 0x35 0x31 } \\
\text { 0x2E 0x32 0x20 0x41 } \\
\text { 0x63 0x63 0x65 0x6C } \\
\text { 0x65 0x72 0x6F 0x6D } \\
\text { 0x65 0x74 0x65 0x72 } \\
\text { 0x5F 0x53 0x65 0x6E } \\
\text { 0x73 0x6F 0x72 }\end{array}$ \\
\hline- & Checksum & Soma dos Octetos dentro da tabela $T E D S$. & 2 & U16 & $0 \times 550 \times 54$ \\
\hline
\end{tabular}

A especificação dos PHY TEDS não é feita pela norma IEEE1451.0 e sim pelas normas relativas à conexão física de comunicação, no caso IEEE1451.2 e IEEE1451.5. 
Tabela 3.9 - User's Transducers Name TEDS do WTIM.

\begin{tabular}{|c|c|c|c|c|c|}
\hline \multicolumn{6}{|c|}{ User's Transducers Name - WTIM } \\
\hline 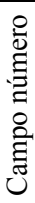 & Nome & Descrição & $\begin{array}{l}\text { Comprimento } \\
\text { do Campo } \\
\text { (Bytes) }\end{array}$ & 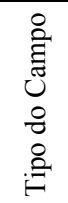 & Conteúdo do Campo \\
\hline & & \multicolumn{4}{|c|}{ Informações relacionadas à Estrutura de Dados } \\
\hline- & Lenght & Número de octetos para descrever o $T E D S$. & 4 & U32 & 0x00 0x00 0x00 0x26 \\
\hline 3 & TEDSID & Identificação do TEDS. & 4 & U32 & 0x00 0x0C 0x01 0x01 \\
\hline 4 & Format & $\begin{array}{l}\text { Define o formato de representação do } \\
\text { TCName. O TCName descreve o nome do } \\
\text { TIM de acordo com o padrão definido no } \\
\text { formato. }\end{array}$ & 1 & U8 & $0 \mathrm{x} 00$ \\
\hline 5 & TCName & Nome do WTIM. & - & - & $\begin{array}{l}\text { 0x49 0x45 0x45 0x45 } \\
\text { 0x31 0x34 0x35 0x31 } \\
\text { 0x2E 0x35 0x20 0x45 } \\
\text { 0x43 0x47 0x5F 0x42 } \\
\text { 0x69 0x6F 0x70 0x6F } \\
\text { 0x74 0x65 0x6E 0x63 } \\
\text { 0x69 0x61 0x6C 0x5F } \\
\text { 0x53 0x65 0x6E 0x73 } \\
\text { 0x6F 0x72 }\end{array}$ \\
\hline- & Checksum & Soma dos Octetos dentro da tabela $T E D S$. & 2 & U16 & $0 \times \mathrm{xF} 40 \times 85$ \\
\hline
\end{tabular}

\subsubsection{Mensagens e comandos}

Para a comunicação entre o TIM e o $N C A P$, a norma IEEE1451.0 utiliza mensagens com estruturas definidas passadas entre dispositivos através dos módulos de comunicação (TORRI, 2008). Três tipos de mensagens são definidas.

- Command: são representados por comandos enviados pelo $N C A P$ aos TIMs. Permitem a leitura e escrita de TEDS e de dados, além da configuração e controle dos módulos transdutores. Dentro da norma existem os comandos padrões e os definidos pelo próprio fabricante.

- Command Reply: são as respostas originadas nos TIMs com destino ao $N C A P$.

- TIM Initiated Message: são comandos que partem diretamente dos TIMs ao NCAP.

As mensagens de TIM Initiated Message somente ocorrem no início de conexão dos TIMs com o NCAP. Nessas mensagens são encaminhas informações das 
características dos TIMs. Numa analogia, pode-se comparar a conexão desses TIMs a inicialização de um dispositivo USB em um computador. No qual o dispositivo encaminha algumas informações ao computador que, por sua vez, reconhece o dispositivo de modo plug and play e o instala no computador.

Com exceção da primeira mensagem, o restante das mensagens é realizada através da dupla Command e Command Reply. Nesse sentido, destaca-se que tanto o STIM como o WTIM são transdutores que respondem a requisições realizadas pelo operador. Sendo que a estrutura das mensagens de requisição de comando foi detalhada tal como Higuera et al. (2009) apresenta.

\subsubsection{API IEEE1451.0}

A API do protocolo IEEE1451.0 define uma lista de interfaces para ler e escrever os TransducerChannels, ler e escrever o TEDS do transdutor, enviar informações de configuração, controle e comandos aos TIMs (ver figura 3.11) (IEEE, 2007a) (SONG; LEE, 2006).

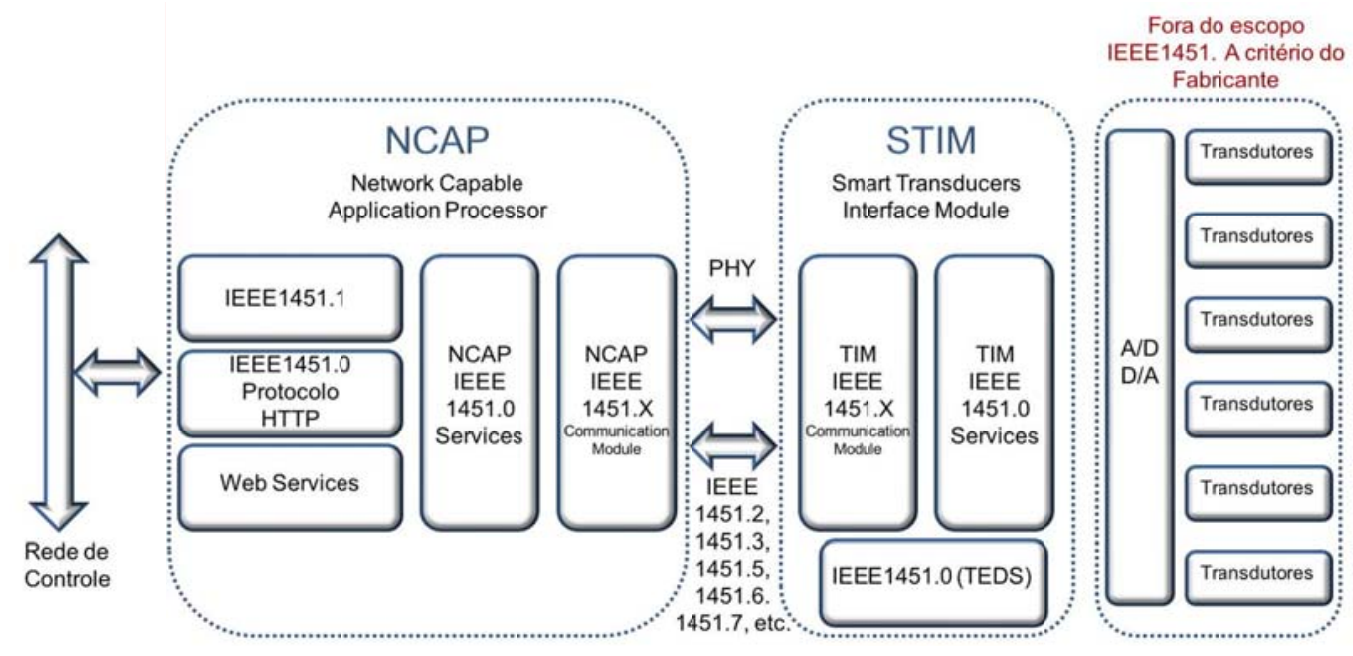

Figura 3.11 - Modelo de referência IEEE1451.0.

Dentro da norma são propostas duas APIs que foram incorporadas nesse projeto com o intuito de facilitar a concepção modular do sistema. A primeira $A P I$, a 
TransdutorServices foi utilizada para o controle e acesso de comandos e dados. Dentre seus métodos estão o de leitura e escrita de TransducerChannels e TEDS, além das operações de configuração, controle e comando para o TIM. Já o Module Communication possui uma interface de comunicação entre NCAP e TIM.

A partir da $A P I$ foram projetadas as funções que permitem a dinâmica do IEEE1451.0, IEEE1451.2 e IEEE1451.5. Dentre essas funções destacam-se as das classes TEDSManager, em específico, o método readTEDS; TransducerAccess, com a função de readData; e os métodos da CommunicationModules. Os resultados e a estrutura desses objetos são apresentados nas figuras 4.15, 4.17, 4.18 e 4.22 do capítulo 4.

\subsection{Desenvolvimento e implementação do IEEE1451.2}

Como já discutido no capítulo 2, a norma IEEE1451.2 publicada em 1997 definia uma interface digital de 10 fios para a comunicação entre transdutores e microprocessadores (SONG; LEE, 2008b). A parte do transdutor era reconhecida como STIM, e a do microprocessador NCAP. A camada física de comunicação, $T I I$, incorporava o protocolo SPI com linhas de sincronização de dados.

A dificuldade em se trabalhar com uma interface de múltiplos fios imobilizou o avanço de implementações da norma IEEE1451.2. Desde a publicação da norma pesquisadores investiram em outras formas de interface de comunicação. Contudo não compatibilizaram a norma em questão com a IEEE1451.0. A análise do protocolo em questão utilizou a atualização descrita em Song e Lee (2008b). Nesse trabalho a norma IEEE1451.2 incorpora os atributos da IEEE1451.0 e realiza a interface entre o $S T I M$ e o $N C A P$ através de uma comunicação serial $R S-232$ (ANEXO H). O principal ponto para a atualização da norma está relacionado ao preenchimento do PHY TEDS, conforme a atualização proposto por Song e Lee (2008b). Os campos desse TEDS seguem abaixo:

- TEDSID: os valores configurados foram, respectivamente, 0x05, 0x0D 0x01 e 0x01. Esses valores configuram o TEDS para a norma IEEE1451.5, 
com classe $P H Y$ TEDS e para o formato atualizado da versão IEEE1451 formado somente por octetos.

- Serial: esse campo recebeu o valor 0x06, referente à possibilidade de inclusão da interface de comunicação USB.

- MaxBPS: o protocolo USB do AT90USB162 foi configurado com máxima taxa de transmissão de 10Mbps.

- MaxCDev: esse campo configura o número máximo de dispositivos conectados ao módulo. Como somente há um nó STIM esse campo foi preenchido com o valor $0 \mathrm{x} 01$.

- MaxRDev: similar ao anterior esse campo configura o número máximo de dispositivos que funcionam simultaneamente ao módulo. $\mathrm{O}$ valor preenchido também foi 0x01.

-Encrypt: como a transmissão USB não utiliza nenhuma criptografia para transmissão de dados esse campo ficou configurado com valor zero.

- Authent: também não se utilizou nenhuma autenticação. Esse campo foi configurado com o valor zero.

- MinKeyL: esse campo armazena a menor chave de criptografia. Como não se utilizou esse atributo, o campo ficou com valor zero.

- MaxKeyL: esse campo armazena a maior chave de criptografia. Como não se utilizou esse atributo, o campo ficou com valor zero.

- MaxSDU: o máximo tamanho de comando (Service Data Unit) que é transferido entre o módulo foi configurado como 255 bits.

- MinALat: o mínimo período de latência, em nanosegundos, para realizar a primeira transmissão a um dispositivo foi configurado em meio segundo.

- MinTLat: para transmitir o menor pacote entre os nós foi configurado o valor de $1 \mu \mathrm{s}$. 
Tabela 3.10 - PHY TEDS do STIM.

\begin{tabular}{|c|c|c|c|c|c|}
\hline \multicolumn{6}{|c|}{ PHY TEDS - STIM } \\
\hline 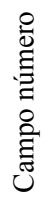 & Nome & Descrição & $\begin{array}{l}\text { Comprimento } \\
\text { do Campo } \\
\text { (Bytes) }\end{array}$ & 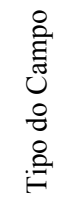 & $\begin{array}{l}\text { Conteúdo do } \\
\text { Campo }\end{array}$ \\
\hline & & $\begin{array}{l}\text { Informações relacionadas à Estrutura de } \\
\text { Dados }\end{array}$ & & & \\
\hline- & Lenght & Número de octetos para descrever o $T E D S$. & 4 & $\mathrm{U} 32$ & $\begin{array}{l}\text { 0x00 0x00 0x00 } \\
0 \times 33\end{array}$ \\
\hline 3 & TEDSID & Identificação do $T E D S$. & 4 & U32 & $\begin{array}{l}\text { 0x02, 0x0D 0x01 } \\
\text { 0x01 }\end{array}$ \\
\hline 10 & Serial & Tipo de interface serial. & 1 & U8 & $0 \times 06$ \\
\hline 11 & MaxBPS & Máxima transmissão de dados. & 4 & $\mathrm{U} 32$ & $\begin{array}{l}\text { 0x00 0x98 0x96 } \\
0 \times 80\end{array}$ \\
\hline 12 & MaxCDev & $\begin{array}{l}\text { Número máximo de dispositivos que podem } \\
\text { trabalhar simultaneamente. }\end{array}$ & 2 & U16 & 0x00 0x01 \\
\hline 13 & MaxRDev & $\begin{array}{l}\text { Número máximo de dispositivos que podem se } \\
\text { registrar simultaneamente. }\end{array}$ & 2 & U16 & $0 \times 000 \times 01$ \\
\hline 14 & Encrypt & Criptografia da informação. & 2 & U16 & 0x00 0x00 \\
\hline 15 & Authent & Suporte a autenticação. & 2 & U16 & $0 \times 000 \times 00$ \\
\hline 16 & $\operatorname{MinKeyL}$ & Menor chave de segurança. & 2 & U16 & $0 \times 000 \times 00$ \\
\hline 17 & $\operatorname{MaxKeyL}$ & Maior chave de segurança. & 2 & U16 & $0 \times 000 \times 00$ \\
\hline 18 & $\operatorname{MaxSDU}$ & Maior palavra que pode ser transmitida. & 2 & U16 & $0 \mathrm{x} 000 \mathrm{xFF}$ \\
\hline 19 & MinALat & $\begin{array}{l}\text { Tempo mínimo para iniciar a primeira } \\
\text { transmissão com um dispositivo desconectado. }\end{array}$ & 4 & U32 & $\begin{array}{l}\text { 0x1D 0xCD 0x65 } \\
\text { 0x00 }\end{array}$ \\
\hline 20 & MinTLat & Tempo para transmitir a menor palavra. & 1 & $\mathrm{U} 32$ & $\begin{array}{l}\text { 0x00 0x00 0x03 } \\
\text { 0xE8 }\end{array}$ \\
\hline 21 & MaxXact & $\begin{array}{l}\text { Número máximo de comandos incompletos na } \\
\text { fila. }\end{array}$ & 1 & U8 & $0 \times 01$ \\
\hline 22 & Battery & Conexão com bateria ou fonte externa. & 1 & U8 & $0 \times 00$ \\
\hline 23 & Version & Versão do rádio. & 2 & U16 & $0 \times 000 \times 01$ \\
\hline 24 & MaxRetry & $\begin{array}{l}\text { Número de tentativas para transmissão de } \\
\text { comandos. }\end{array}$ & 2 & U16 & 0x00 0x05 \\
\hline 41 & Baud & Taxa de transmissão de dados. & 4 & U32 & $\begin{array}{l}0 \times 000 \times 000 \times 25 \\
0 x 80\end{array}$ \\
\hline 42 & DataBits & Bits transmitidos em uma palavra. & 1 & U16 & 0x00 0x08 \\
\hline 43 & Parity & $\begin{array}{l}\text { Paridade para verificação da integridade do } \\
\text { dado. }\end{array}$ & 1 & U16 & $0 \times 000 \times 00$ \\
\hline 44 & StopBit & Bit de parada. & 1 & U16 & $0 \times 000 \times 01$ \\
\hline 45 & Terminator & Finalizador da frase. & 1 & U16 & $0 \times 000 \times 00$ \\
\hline- & Checksum & Soma dos Octetos dentro da tabela $T E D S$. & 2 & U16 & $0 \times \mathrm{xFA} 0 \times 17$ \\
\hline
\end{tabular}


- MaxXact: a quantidade máxima de transações simultâneas no STIM foi configurada como o valor $0 \mathrm{x} 01$.

- Battery: como o módulo funciona, prioritariamente, ligado a uma fonte externa e não uma bateria, esse campo foi configurado com o valor 0x00.

- RadioVer: a versão do módulo foi configurada com o valor 0x01, correspondendo a v.1.

- MaxRetry: nesse campo foi configurada a máxima quantidade de tentativas para envio de um comando. No caso após cinco tentativas há uma desconexão no canal de comunicação.

- Baud: para a configuração baudrate, foi imposto o valor 9600, taxa comumente utilizada em transmissões seriais.

- DataBits: o valor de número de bits por palavra foi configurado em 8 bits.

- Parity: como não há verificação de paridade, foi configurado o valor zero.

- Stopbits: foi configurado somente um bit de parada, com valor 0x01.

- Terminator: não foi utilizado bit de terminação.

- Checksum: valor calculado de acordo com a equação 3.15 .

Nesse trabalho ao invés da comunicação serial $R S-232$, conforme Song e Lee (2008b), foi proposta a interface a partir de um barramento USB. Tal modificação não altera a configuração do valor de PHY TEDS e das interfaces com o IEEE1451.0 propostas pelos pesquisadores do NIST. Esse fato é exemplificado pela tabela 3.10, que apresenta a descrição do $P H Y$ TEDS similar ao apresentado no trabalho dos autores citados (ANEXO H).

A proposta desse trabalho foi a de estender a atualização com a incorporação de uma camada física diferente, com maiores possibilidades de tornar o sensor um sistema totalmente plug and play, conforme o espírito de funcionamento dos instrumentos e equipamentos com comunicação USB. 


\subsection{Desenvolvimento e implementação do IEEE1451.5}

A norma IEEE1451.5, como apresentada no capítulo anterior, estabelece um padrão para comunicação de redes sem fío. Um dos pontos da norma é o estabelecimento do WTIM, que une os conceitos da norma IEEE1451.0 com o IEEE1451.5. Outra característica é o PHY TEDS que armazena as configurações de comunicação e interface física.

As conexões entre a norma IEEE1451.0 e as normas IEEE1451.2 e IEEE1451.5 são realizadas através dos Communications Modules. Esses módulos permitem a interface entre o STIM e WTIM com o NCAP.

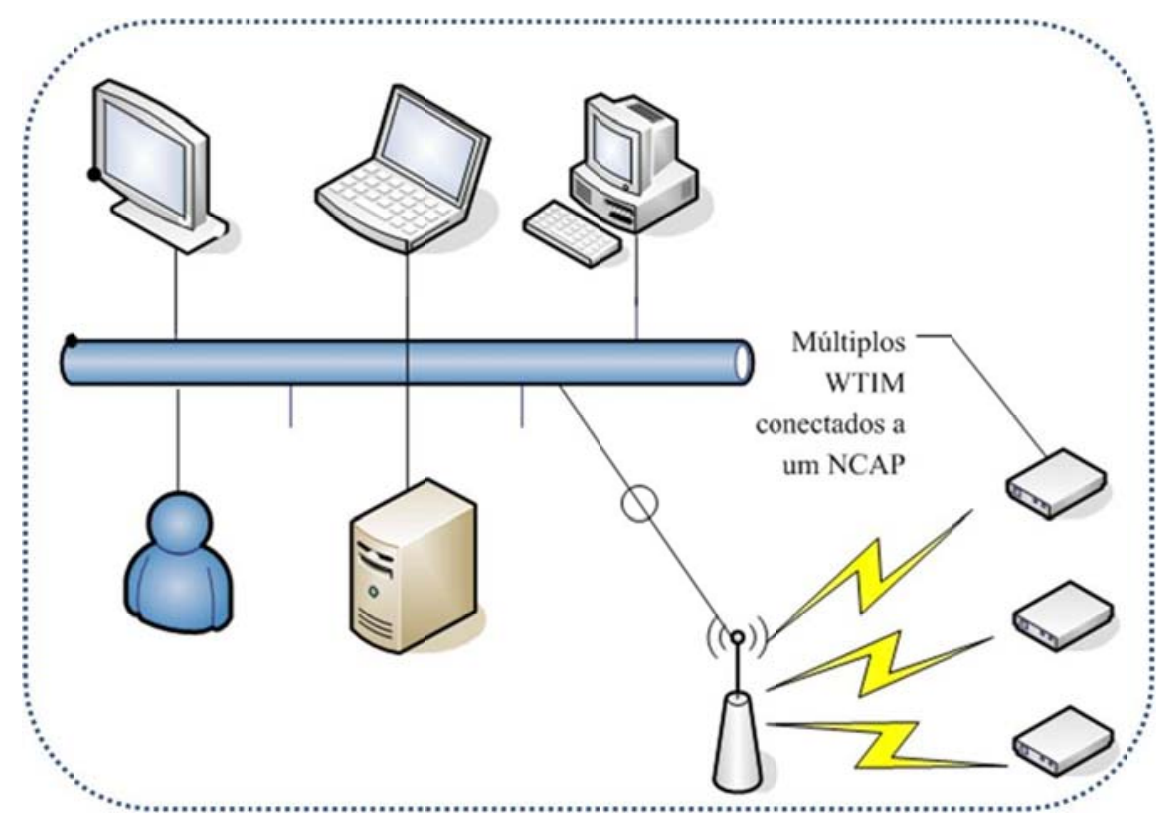

Figura 3.12 - Conexão direta entre WTIM e NCAP.

Dentro da norma IEEE1451.5 há possibilidade para distintas maneiras de conexão entre os WTIMs e o NCAP. Nesse projeto, selecionou-se uma comunicação que possibilite um NCAP registrar vários WTIMs, conforme figura 3.12.

Para o registro do PHY TEDS, conforme o Anexo I, o WTIM do projeto se restringiu a especificar somente os campos obrigatórios apresentados na tabela 3.11. 
Tabela $3.11-P H Y$ TEDS do WTIM.

\begin{tabular}{|c|c|c|c|c|c|}
\hline \multicolumn{6}{|c|}{ PHY TEDS - WTIM } \\
\hline 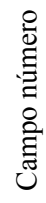 & Nome & Descrição & $\begin{array}{c}\text { Comprimento } \\
\text { do Campo } \\
\text { (Bytes) }\end{array}$ & 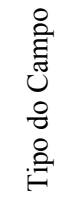 & $\begin{array}{l}\text { Conteúdo do } \\
\text { Campo }\end{array}$ \\
\hline & & $\begin{array}{l}\text { Informações relacionadas à Estrutura de } \\
\text { Dados }\end{array}$ & & & \\
\hline- & Lenght & Número de octetos para descrever o TEDS. & 4 & U32 & $\begin{array}{l}\text { 0x00 0x00 0x00 } \\
0 \times 33\end{array}$ \\
\hline 3 & TEDSID & Identificação do $T E D S$. & 4 & $\mathrm{U} 32$ & $\begin{array}{l}\text { 0x00 0x01 0x01 } \\
\text { 0x01 }\end{array}$ \\
\hline 10 & Radio & Tipo de rádio comunicador. & 1 & U8 & $0 \mathrm{x} 00$ \\
\hline 11 & $M a x B P S$ & Máxima transmissão de dados. & 4 & U32 & $\begin{array}{l}\text { 0x03 0x37 0xF9 } \\
0 \times 80\end{array}$ \\
\hline 12 & MaxCDev & $\begin{array}{l}\text { Número máximo de dispositivos que podem } \\
\text { trabalhar simultaneamente. }\end{array}$ & 2 & U16 & 0x00 0x01 \\
\hline 13 & MaxRDev & $\begin{array}{l}\text { Número máximo de dispositivos que podem se } \\
\text { registrar simultaneamente. }\end{array}$ & 2 & U16 & 0x00 0x01 \\
\hline 14 & Encrypt & Criptografia da informação. & 2 & U16 & $0 \times 000 \times 00$ \\
\hline 15 & Authent & Suporte a autenticação. & 2 & U16 & 0x00 0x00 \\
\hline 16 & MinKeyL & Menor chave de segurança. & 2 & U16 & 0x00 0x00 \\
\hline 17 & $M a x K e y L$ & Maior chave de segurança. & 2 & U16 & 0x00 0x00 \\
\hline 18 & $\operatorname{MaxSDU}$ & Maior palavra que pode ser transmitida. & 2 & U16 & 0x00 0xFF \\
\hline 19 & MinALat & $\begin{array}{l}\text { Tempo mínimo para iniciar a primeira } \\
\text { transmissão com um dispositivo desconectado. }\end{array}$ & 4 & U32 & $\begin{array}{l}\text { 0x1D 0xCD 0x65 } \\
0 \times 00\end{array}$ \\
\hline 20 & MinTLat & Tempo para transmitir a menor palavra. & 1 & $\mathrm{U} 32$ & $\begin{array}{l}\text { 0x00 0x00 0x03 } \\
\text { 0xE8 }\end{array}$ \\
\hline 21 & MaxXact & $\begin{array}{l}\text { Número máximo de comandos incompletos na } \\
\text { fila. }\end{array}$ & 1 & U8 & $0 \times 01$ \\
\hline 22 & Battery & Conexão com bateria ou fonte externa. & 1 & U8 & $0 \times 00$ \\
\hline 23 & RadioVer & Versão do rádio. & 2 & U16 & 0x00 0x01 \\
\hline 24 & MaxRetry & $\begin{array}{l}\text { Número de tentativas para transmissão de } \\
\text { comandos. }\end{array}$ & 2 & U16 & 0x00 0x05 \\
\hline- & Checksum & Soma dos Octetos dentro da tabela $T E D S$. & 2 & U16 & 0xFA 0xD4 \\
\hline
\end{tabular}

Para o PHY TEDS é digno de nota a determinação da comunicação Wifi. Os detalhes de especificações desse TEDS seguem abaixo:

- TEDSID: os valores configurados foram, respectivamente: 0x05, 0x0D, 0x01 e 0x01. Esses valores configuram o TEDS para a norma IEEE1451.5, 
com classe $P H Y$ TEDS e para o formato atualizado da versão IEEE1451 formado somente por octetos.

- Radio: esse campo recebeu o valor zero para enumerar a interface de comunicação IEEE802.11.

- MaxBPS: o protocolo Wifi IEEE802.11g tem máxima taxa de transmissão de $54 M b p s$.

-MaxCDev: esse campo configura o número máximo de dispositivos conectados ao módulo. Como somente há um nó WTIM esse campo foi preenchido com o valor $0 \mathrm{x} 01$.

- MaxRDev: similar ao anterior esse campo configura o número máximo de dispositivos que funcionam simultaneamente ao módulo. $\mathrm{O}$ valor preenchido também foi 0x01.

-Encrypt: como a transmissão Wifi não utilizou nenhuma criptografia para transmissão de dados esse campo ficou configurado com valor zero.

- Authent: também não se utilizou nenhuma autenticação. Esse campo foi configurado com o valor zero.

- MinKeyL: esse campo armazena a menor chave de criptografia. Como não se utilizou esse atributo, o campo ficou com valor zero.

- MaxKeyL: esse campo armazena a maior chave de criptografia. Como não se utilizou esse atributo, o campo ficou com valor zero.

- MaxSDU: o máximo tamanho de comando (Service Data Unit) que é transferido pelo módulo foi configurado como 255 bits.

- MinALat: o mínimo período de latência, em nanosegundos, para realizar a primeira transmissão a um dispositivo foi configurado em meio segundo.

- MinTLat: para transmitir o menor pacote entre nós foi configurado o valor de $1 \mu$ s (valor estimado).

- MaxXact: a quantidade máxima de transações simultâneas no WTIM foi configurado como o valor $0 \mathrm{x} 01$. 
- Battery: como o módulo funciona, prioritariamente, ligado a uma fonte externa e não uma bateria, esse campo foi configurado com o valor 0x00.

- RadioVer: a verão do módulo foi configurada com o valor 0x01, correspondendo a v.1.

- MaxRetry: nesse campo foi configurado a máxima quantidade de tentativas para envio de um comando. No caso após cinco tentativas há uma desconexão no canal de comunicação.

- Checksum: valor calculado de acordo com a equação 3.15 .

\subsection{Framework Axis para Web Services}

O AXIS (Apache EXtensible Interaction System) é definido pela Apache como um mecanismo SOAP. Ele foi utilizado como biblioteca para o desenvolvimento de Consumidores de Serviços e de Provedores de Serviços, em outras palavras, de um cliente de serviços $S O A P$ e de um servidor para executar serviços acessíveis pelo SOAP (OLIVEIRA, 2005).

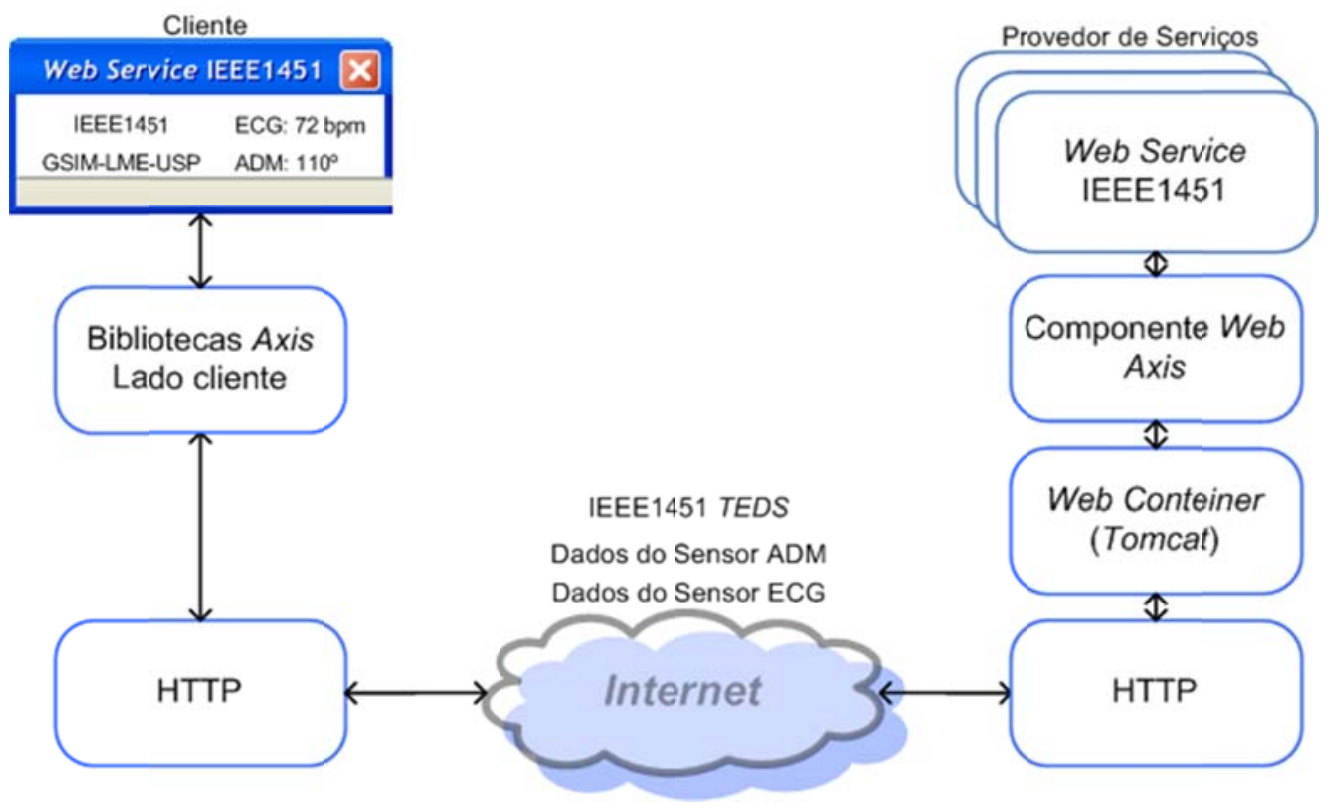

Figura 3.13 - Arquitetura de acesso remoto através de Web Services. 
Para a programação do cliente, o Axis fornece uma API para invocar serviços do SOAP RPC, a possibilitar o envio e o recebimento de mensagens. No lado do servidor, são fornecidos mecanismos para escrever e acessar serviços $R P C$ ou serviços de mensagens hospedadas por um Web Conteiner.

Além de funções de suporte para o $S O A P$, o Axis também inclui suporte extensivo para $W S D L$, bem como ferramentas que geram classes Java a partir de um WSDL. Para o desenvolvimento de um provedor de Web Services que disponibiliza as informações relativas aos sensores de ECG e de ADM foi utilizado o Apache Axis2 em conjunto com o Web Container Apache Tomcat versão 6.0, além do ambiente integrado de desenvolvimento Eclipse Helios Service Release 1 com o Java Development Kit (JDK) 1.6.0. Todos esses componentes e programas, como visto na figura 3.13, rodando em um sistema operacional Windows Xp.

$\mathrm{O}$ acesso remoto foi feito através do método Request-Response Messaging, no qual o cliente faz uma solicitação ao Web Service, que executa um processamento relativo a informações do $T E D S$ dos sensores e dos dados coletados de frequência cardíaca ou de graus, no caso da amplitude de movimento. O contêiner intercepta as requisições e as repassa ao programa $N C A P$. Após o processamento, o contêiner envia a resposta ao cliente.

A definição dessa plataforma se deu devido ao fato de ser possível, com o Axis, transformar qualquer classe Java em um Web Service. Dessa maneira as classes que apresentam as informações dos sensores e dos dados de ECG e de ADM se tornaram um serviço, e seus métodos públicos, as operações do serviço. Permitindo a um cliente remoto o acesso das informações coletadas dos sensores biomédicos através da Internet. 


\section{RESULTADOS}

Nesse capítulo são apresentados os resultados do desenvolvimento e integração da rede de sensores biomédicos utilizando o protocolo IEEE1451. Para tanto são expostos os seguintes tópicos: simulação e análise do circuito condicionador de biopotenciais, bem como a caracterização da placa desenvolvida; caracterização dos acelerômetros como inclinômetros e testes com o aparato preso ao braço e antebraço; apresentação do desenvolvimento dos sistemas microcontrolados; o desenvolvimento dos nós IEEE1451.0, IEEE1451.2 e IEEE1451.5, demonstrando o perfil da interface com o usuário e o funcionamento do Web Service IEEE1451; e, por último, a validação com o framework proposto por Song e Lee (2006) (2007).

\subsection{Simulação e análise do circuito de condicionamento de biopotenciais}

Como demonstrado no capítulo 3, o projeto de um circuito condicionador de biopotenciais, em específico de ECG, deve ter como objetivo amplificar valores de tensão na faixa de $100 \mu \mathrm{V}$ até $4 \mathrm{mV}$, com banda de até $250 \mathrm{~Hz}$. Contudo para um medidor de frequência cardíaca, foi especificado uma tensão no eletrodo de aproximadamente $1 \mathrm{mV}$ e uma faixa de $100 \mathrm{~Hz}$ para o sinal. Nesse sentido foi requisito do desenvolvimento do circuito, a simulação dos filtros anteriormente projetados.

Para a simulação dos filtros e do circuito com os amplificadores (diferencial, não inversor e somador) foi utilizado o programa Multisim 10 versão estudantil. Esse software além de permitir análise SPICE (Simulation Program with Integrated Circuit Emphasis) possui instrumentos virtuais que possibilitam acompanhar, tal como uma bancada virtual, a análise transiente de circuitos.

Os três circuitos críticos que foram simulados separadamente foram: o filtro passa-altas de $2^{\mathrm{a}}$ ordem Butterworth, o filtro notch duplo T e o filtro passa-baixas de $4^{\mathrm{a}}$ ordem Butterworth. A começar pelo filtro passa-altas, desenhou-se o esquema de 
tal forma a obter as condições mais próximas da construção do circuito. Como demonstrado na figura 4.1, o amplificador operacional LF353 foi alimentado com tensão simétrica de $5 \mathrm{~V}$.

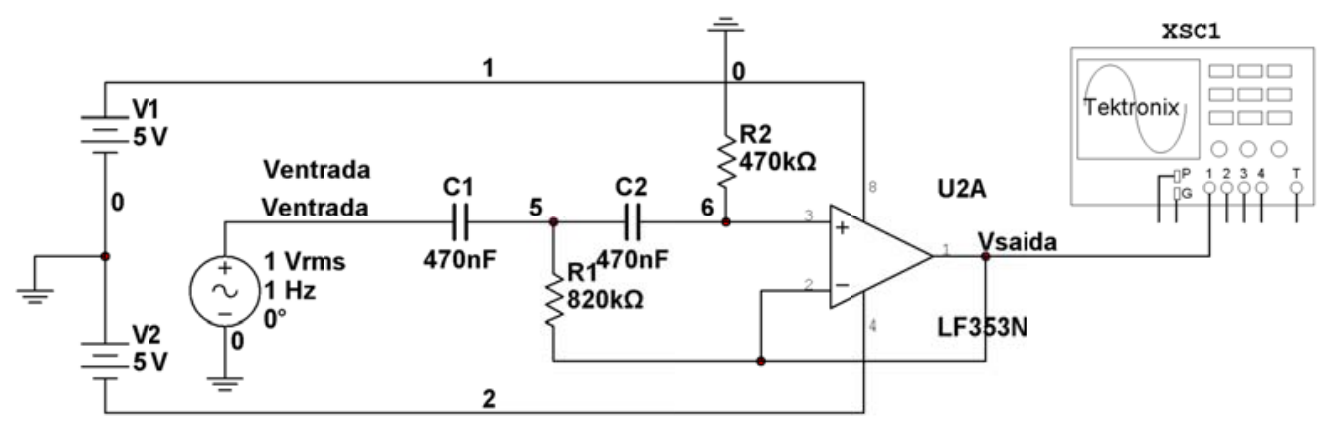

Figura 4.1 - Esquema elétrico do filtro passa-altas de $2^{\mathrm{a}}$ ordem.

A partir da análise AC foi possível visualizar a curva de resposta em frequência do módulo e fase do filtro. O cálculo do ganho de tensão foi obtido através da eq.(4.1). O simulador Multisim realizou um levantamento de pontos de tensão de saída em relação à tensão de entrada. Através dessas curvas foram analisados os comportamentos dos sinais em função da variação da frequência. Além disso, a resposta em frequência do filtro permitiu confirmar o tipo de filtro projetado, o ganho/atenuação na banda de passagem, bem como a frequência de corte, também conhecida como frequência de meia potência.

$$
A_{v}(d B)=20 \times \log \frac{V_{\text {saida }}}{V_{\text {entrada }}}
$$

A figura 4.2 apresenta o resultado da análise AC do filtro passa-altas traçada entre as frequências de $0 \mathrm{~Hz}(D C)$ a $10 \mathrm{~Hz}$. A curva do módulo possibilitou verificar que, de fato, foi projetado um filtro passa-altas, pois há um ganho de tensão conforme se aumenta a frequência . A frequência de corte do filtro (ponto de $-3 \mathrm{~dB}$ ) se encontra na frequência $0,5 \mathrm{~Hz}$, tal como havia sido projetado. Além disso, como se utilizou um filtro ativo não há atenuação na banda passante, o que pode ser visto através do patamar em $0 \mathrm{~dB}$ do módulo da banda de passagem do filtro. 

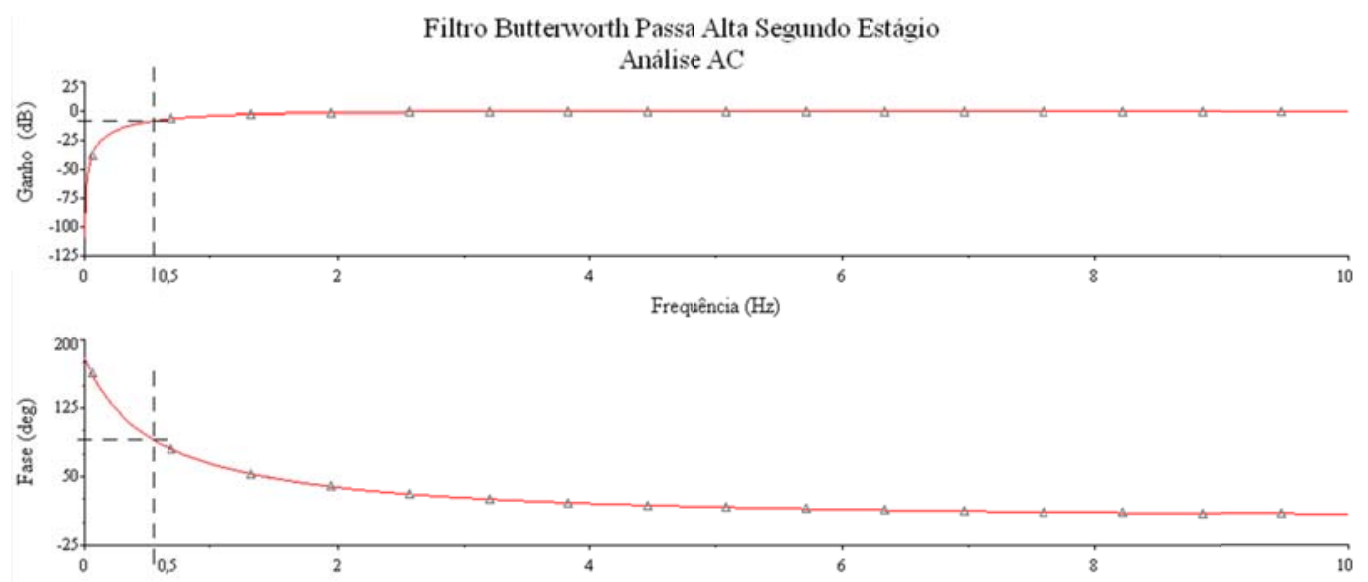

Figura 4.2 - Curva de resposta em frequência do ganho e fase do filtro passa-altas de $2^{\mathrm{a}}$ ordem.

Em relação à fase, nota-se que ela não varia linearmente, porém como já debatido em seção anterior, o filtro do tipo Butterworth não garante resposta em fase linear, em contrapartida possui pouca ondulação na banda passante, requisito primordial para o condicionamento de biopotenciais.

Para a simulação do filtro notch duplo $\mathrm{T}$ foram utilizados os resistores e capacitores projetados na seção anterior, além do amplificador operacional LF353. O esquema elétrico e sua alimentação estão representados na figura 4.3.

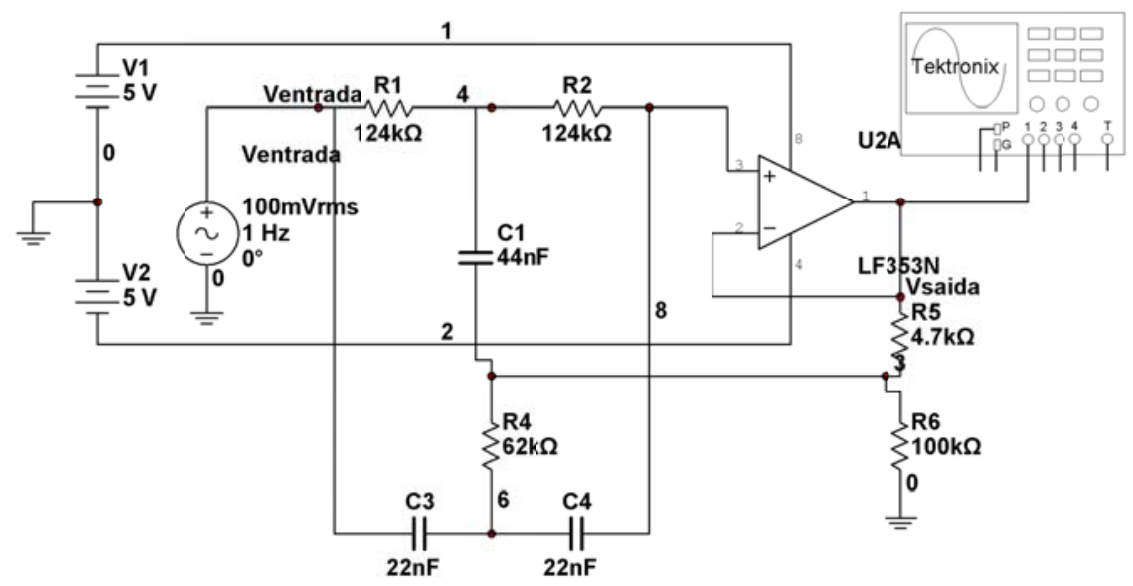

Figura 4.3 - Esquema elétrico do filtro rejeita-faixas. 
$\mathrm{O}$ resultado da análise $\mathrm{AC}$ do circuito pode ser visto na figura 4.4. Nessa figura foi visualizado que o filtro projetado para rejeitar a frequência de $60 \mathrm{~Hz}$ atenua uma faixa maior do que a projetada. Em primeiro lugar, verificou-se que a frequência central $\left(f_{o}\right)$ da banda rejeitada está em $54 \mathrm{~Hz}$ e não em $60 \mathrm{~Hz}$. Esse desvio está ligado a impossibilidade de obter os valores precisos de resistores e capacitores projetados, dado que na prática são utilizados valores comerciais desses componentes passivos.

A banda larga de rejeição do filtro pode ser explicada pela escolha dos valores que ajustam o índice de mérito (Q). Embora o circuito tenha atenuado outras frequências para além do $60 \mathrm{~Hz}$, ele foi levado em consideração na construção do condicionador, pois o filtro possibilitou que o circuito fosse utilizado em países cujo frequência da rede é $50 \mathrm{~Hz}$. Outrossim, o sinal de ECG possui a maior parte do seu conteúdo harmônico até a frequência de $50 \mathrm{~Hz}$, o que para o desenvolvimento de um medidor de frequência cardíaca não traria maiores problemas.

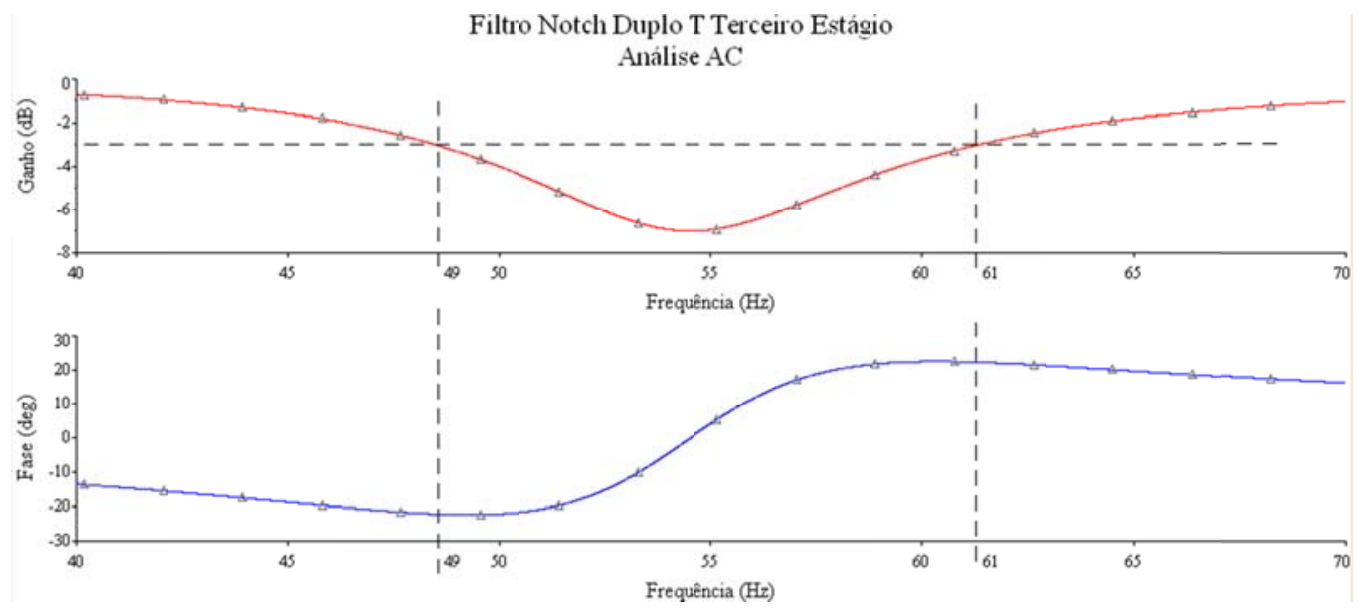

Figura 4.4 - Curva de resposta em frequência do ganho e fase do filtro rejeita-faixas.

A simulação do filtro passa-baixas Butterworth de $4^{\mathrm{a}}$ ordem foi realizada utilizando o CI LF347 e alimentação simétrica de 5V, como visto na figura 4.5. Como resultado da análise $\mathrm{AC}$ obteve-se o diagrama de resposta em frequência da figura 4.6. Através da curva de ganho em função da frequência foi encontrada a frequência de corte $(-3 \mathrm{~dB})$ em $110 \mathrm{~Hz}$. Essa frequência, calculada para $100 \mathrm{~Hz}$ teve esse desvio devido a escolha de componentes comerciais que não possuem o valor 
exato do projeto. Tal fato não prejudicou o desempenho do circuito condicionador de biopotenciais. Apenas apontou para a necessidade de se ter cuidado em relação a escolha dos componentes a serem utilizados na prototipagem do circuito.

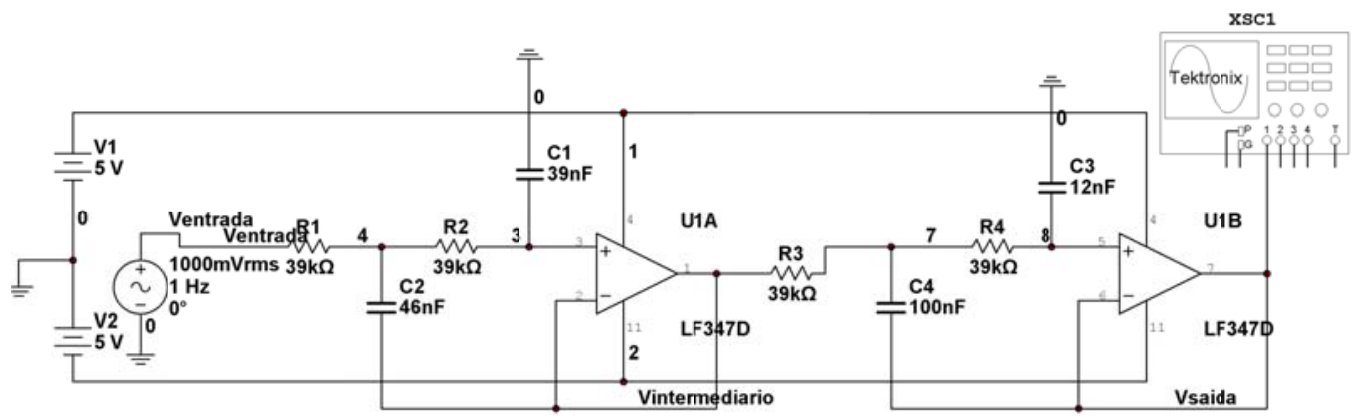

Figura 4.5 - Esquema elétrico do filtro passa-baixas de $4^{\mathrm{a}}$ ordem.
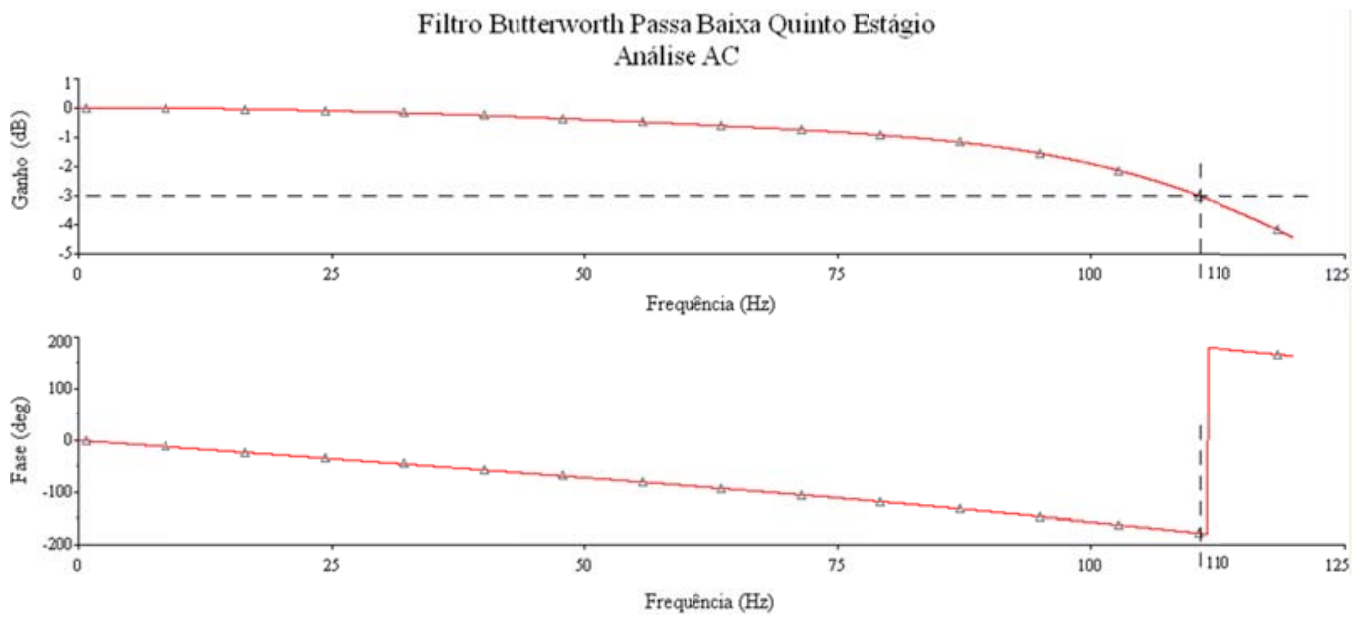

Figura 4.6 - Curva de resposta em frequência do ganho e fase do filtro passa-baixas.

Por último, realizou-se a análise do circuito com todas seus blocos. Nessa análise foram considerados os circuitos amplificadores, bem como os estágios de filtragem e condicionamento (ver Apêndice A). A figura 4.7 apresenta as curvas de ganho e fase do circuito completo. Nessa curva é verificável o comportamento dos filtros passa-altas, rejeita-faixas e passa-baixas atuando em conjunto.

Em relação aos amplificadores foi constatado que a banda de passagem do sinal tem ganho de $60 \mathrm{~dB}$, o que em termos de tensão equivaleria a um ganho de mil 
vezes. Através desse ganho fica viável amplificar sinais na casa de milivolts para a casa da unidade de volts, esse é o caso de um sinal típico de ECG medido através de uma derivação periférica bipolar. Com isso foi possível realizar a amostragem desse sinal utilizando o conversor analógico-digital do microcontrolador ATmega8535 na configuração diferencial. Em relação à fase, nota-se que os filtros passa-altas e rejeita-faixa aplicam não linearidades nas bandas de rejeição e nas proximidades das frequências de corte. Embora isso não afete o circuito para a aplicação pretendida, o cuidado com a fase é necessário quando não se deseja distorções ou assimetrias no sinal.
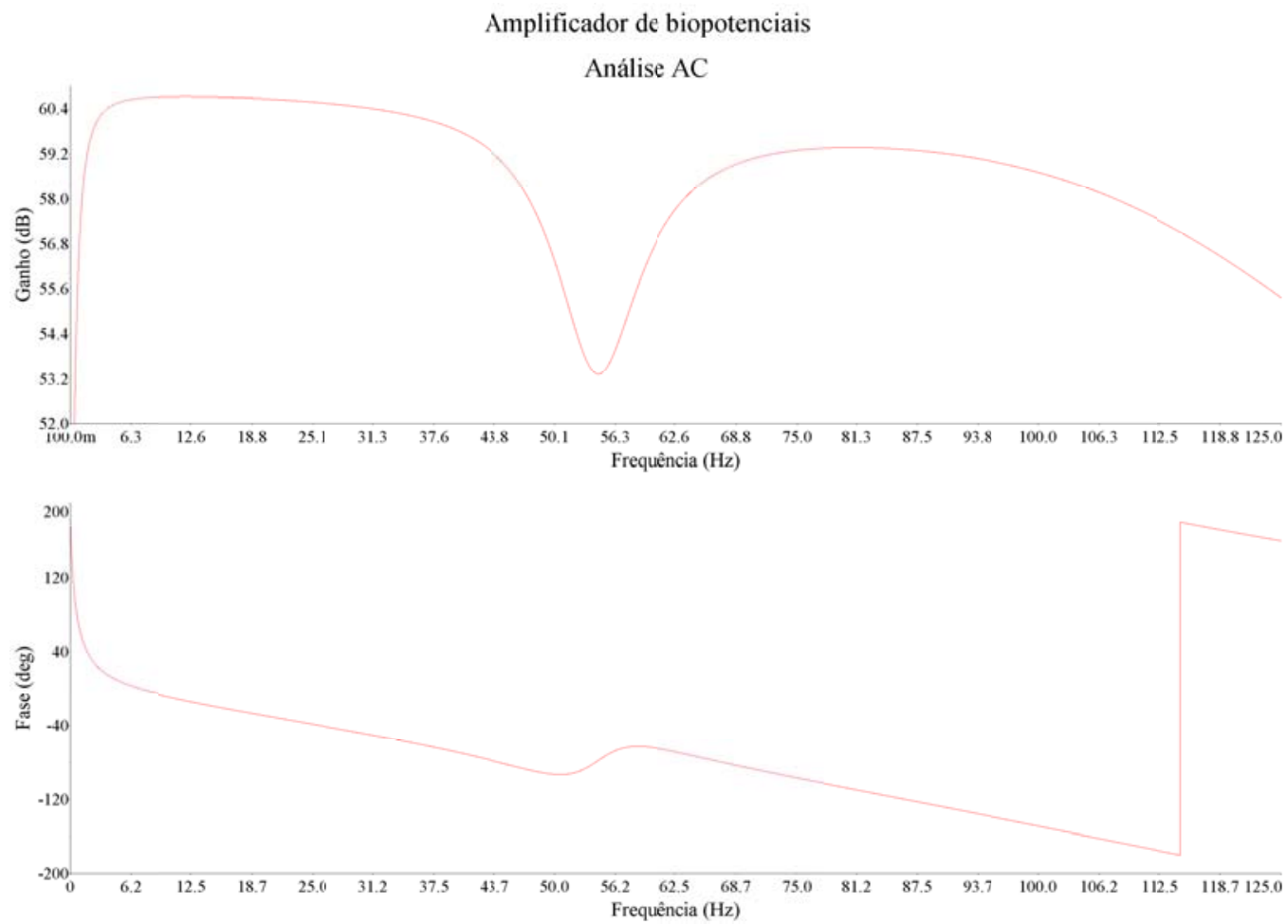

Figura 4.7 - Curva de resposta em frequência do ganho e fase do circuito condicionador de biopotenciais.

Como já mencionado o Multisim permite a utilização de instrumentos virtuais. Para verificação do desempenho do circuito, foram utilizados os instrumentos virtuais de geração de formas de onda Agilent 33120A e o osciloscópio Agilent 54622D. Através do gerador foi selecionada uma onda de ECG padrão, de 
tensão pico a pico $3 \mathrm{mV}$. Na saída do condicionador de biopotenciais foi obtido a mesma onda amplificada em $3 \mathrm{~V}$ pico a pico (ver Apêndice B).

\subsubsection{Caracterização do circuito de condicionamento de biopotenciais}

A placa do circuito condicionador foi projetada no software CadSoft Eagle 4.0 e seu esquema elétrico pode ser visto nos Apêndices C, D e E. O circuito prototipado (figura 4.8) possui dois canais diferenciais. O que, a priori, permitiria realizar medidas de duas derivações simultâneas. Cada canal possui todos os filtros e amplificadores anteriormente apresentados. Além disso, os potenciômetros estão acessíveis externamente através de pequenos orifícios na carcaça metálica. Essa carcaça tem o intuito de blindar o circuito de possíveis interferências eletromagnéticas. As paredes dessa caixa metálica estão ligadas ao sinal de terra do circuito, em outras palavras, na mesma referência utilizada na pulseira de aterramento. Essa pulseira, no caso desse projeto, é conectada na perna esquerda do paciente.
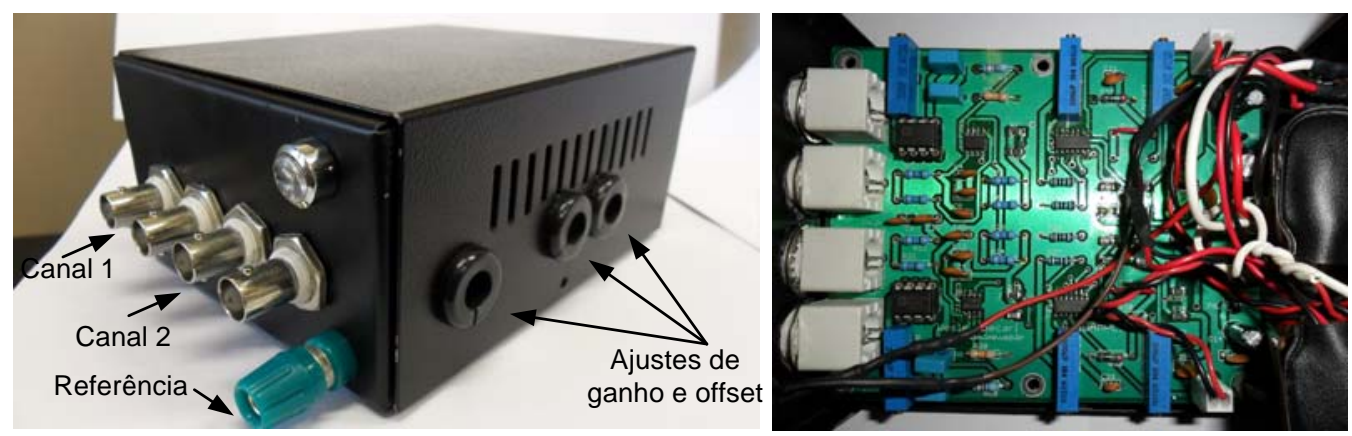

Figura 4.8 - Protótipo do circuito de condicionamento de biopotenciais.

Para caracterizar esse circuito foi utilizado o mesmo procedimento feito durante a simulação. Foi imposta na entrada dos canais uma onda senoidal com tensão pico-a-pico de $20 \mathrm{mV}$. Com isso foi levantada uma curva de resposta em 
frequência para valores de 0,5 a $150 \mathrm{~Hz}$. As medidas de tensão de entrada e de tensão de saída foram obtidas através de um osciloscópio com incerteza de $1 \%$ da medida. O valor do ganho foi calculado utilizando a mesma equação apresentada em eq.(4.1). A curva do ganho em relação à frequência é mostrada na figura 4.9.

Pode-se traçar um paralelo entre a figura 4.7 e a figura 4.9. A primeira corresponde à resposta em frequência da simulação do circuito. Enquanto que a segunda demonstra a caracterização do circuito implementado. Na primeira parte da curva de resposta em frequência é possível verificar o funcionamento do filtro passaaltas tal como projetado. Na banda de passagem do circuito, pode-se constatar um ganho de aproximadamente $58 \mathrm{~dB}$, que corresponde a um ganho de tensão de 800 vezes. Essa configuração, embora um tanto menor do que a simulada, foi ajustada para uma condição em que a medida experimental do sinal de ECG foi considerada ótima. O filtro rejeita-faixas também fica evidenciado na curva de caracterização do circuito. Em especial, verificou-se que nas frequências próximas a $60 \mathrm{~Hz}$ obteve-se um ganho de $54 \mathrm{~dB}$, valor esse $4 \mathrm{~dB}$ abaixo da banda de passagem.

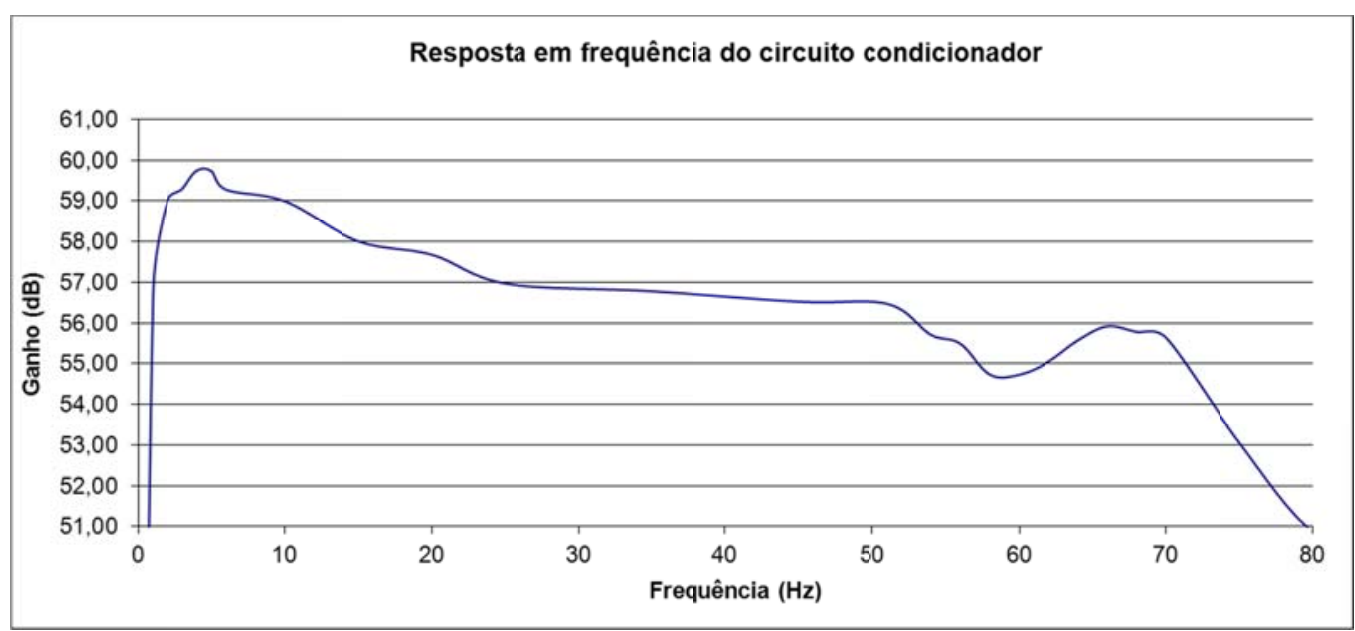

Figura 4.9 - Resposta em frequência do circuito condicionador de biopotenciais.

Por último, verificou-se que o filtro passa-baixas inicia seu corte na frequência de $70 \mathrm{~Hz}$. Esse filtro foi o que mais se distanciou do projeto e da simulação realizada. Pois enquanto nos cálculos foi projetada uma frequência de corte em $100 \mathrm{~Hz}$, na simulação foi vista uma frequência de $110 \mathrm{~Hz}$. Tal fato se deu, 
em larga medida, a incerteza dos valores dos componentes, principalmente dos capacitores, que são fundamentais para a implementação de filtros.

Para demonstrar o funcionamento do circuito de condicionamento, apresentase na figura 4.10 uma medida de ECG. Observa-se nessa medida que a curva $\mathrm{R}$ possui valor de amplitude de, aproximadamente, $1,0 \mathrm{~V}$ e a curva $\mathrm{P}$ possui valor de $0,2 \mathrm{~V}$. Tais valores estão coerentes com o ganho projetado no circuito, pois como visto no capítulo 2 , os valores de amplitude da curva $\mathrm{R}$ e da curva $\mathrm{P}$ são de $1 \mathrm{mV}$ e de $0,2 \mathrm{mV}$, respectivamente. Vale ressaltar que o sinal de ECG medido através da derivação periférica bipolar D1, figura 4.10, é similar ao visto na figura 2.12. O que comprova o funcionamento do condicionador de biopotenciais.

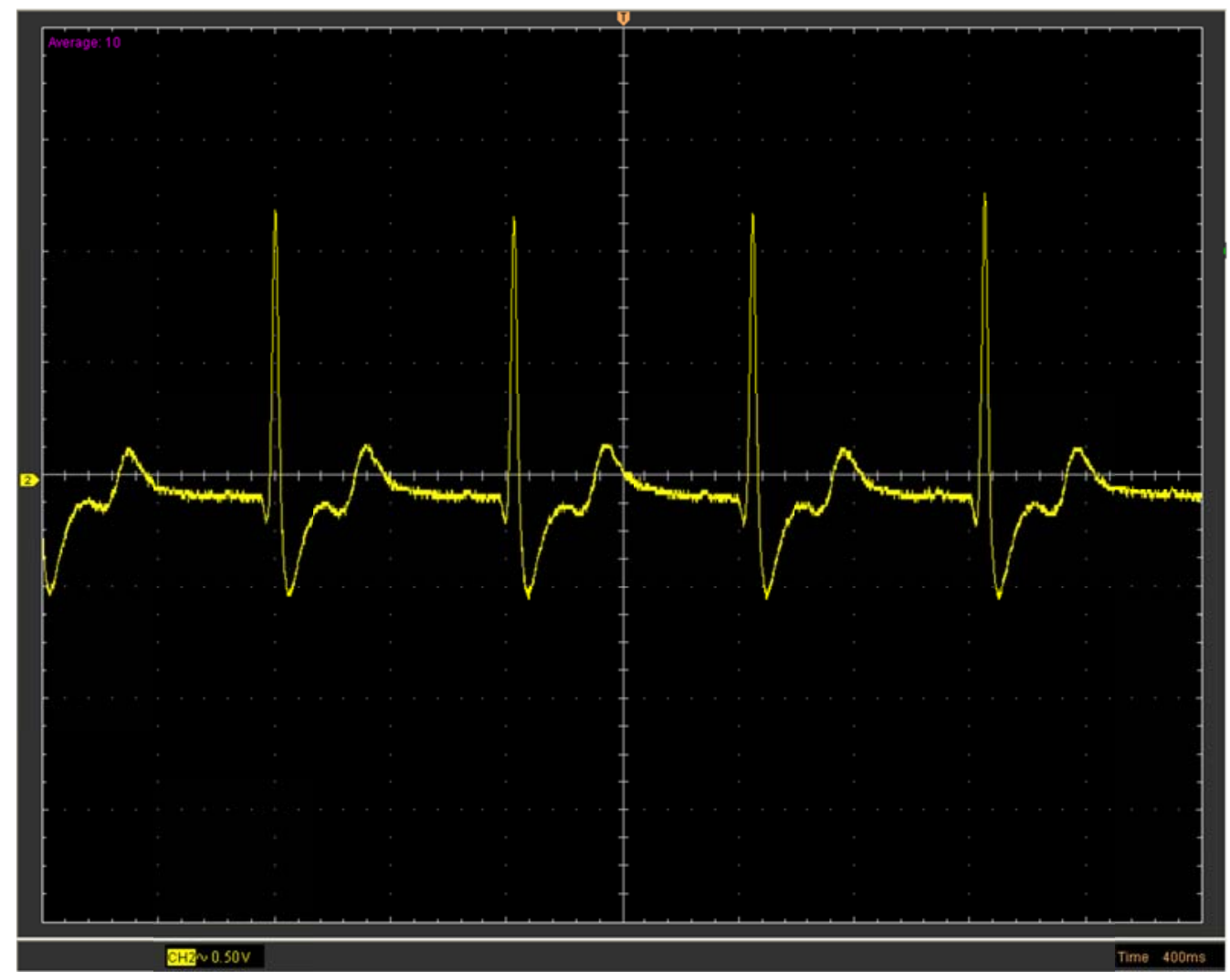

Figura 4.10 - Sinal de ECG condicionado.

Como já discutido anteriormente, a frequência cardíaca de um paciente pode ser medida através do tempo entre duas curvas R. No eletrocardiograma da figura 
4.10 observou-se que as curvas R são periódicas e o tempo entre elas é de $840 \mathrm{~ms}$. Isso corresponde a 71 batimentos por minuto. Através da amostragem do ECG foi possível ao microcontrolador discriminar a onda $\mathrm{R}$ e a partir da contagem de tempo, utilizando um timer, medir e transmitir remotamente o valor da frequência cardíaca.

\subsection{Caracterização dos acelerômetros como inclinômetros}

A caracterização dos acelerômetros MX2125 foi realizada em duas etapas. Primeiramente, confirmou-se a resposta desse sensor para a medida de inclinação em relação ao plano horizontal terra. Após verificar a potencialidade desse sensor como inclinômetro foi utilizada a metodologia apresentada no capítulo 3, cujo enfoque foi de demonstrar uma medida de ADM através da diferença dos ângulos medidos por dois acelerômetros adequadamente alocados no braço e antebraço. Nesse contexto foram obtidos os resultados de medida de ADM entre 20 e $180^{\circ}$.

\subsubsection{Medida de inclinação com um acelerômetro}

De acordo com a folha de dados do acelerômetro MX2125, uma das principais aplicações desse sensor é a medição de inclinação. Essa medida pode ser representada esquematicamente através da figura 4.11. Nessa figura fica explicito o funcionamento do sensor em três condições de inclinação: $0^{\circ}, 30^{\circ}$ e $90^{\circ}$ em relação ao plano de referência.

Se o MX2125 for devidamente montado em uma estrutura mecânica obtém-se o valor da força gravitacional atuando sobre a câmera de gás do acelerômetro. Essa

força é apresentada na figura 4.11 pelos módulos dos vetores normais $\vec{G}_{x}$ e $\vec{G}_{y}$, que estão simplificados pelas letras $\mathrm{G}_{\mathrm{x}}$ e $\mathrm{G}_{\mathrm{y}}$. 


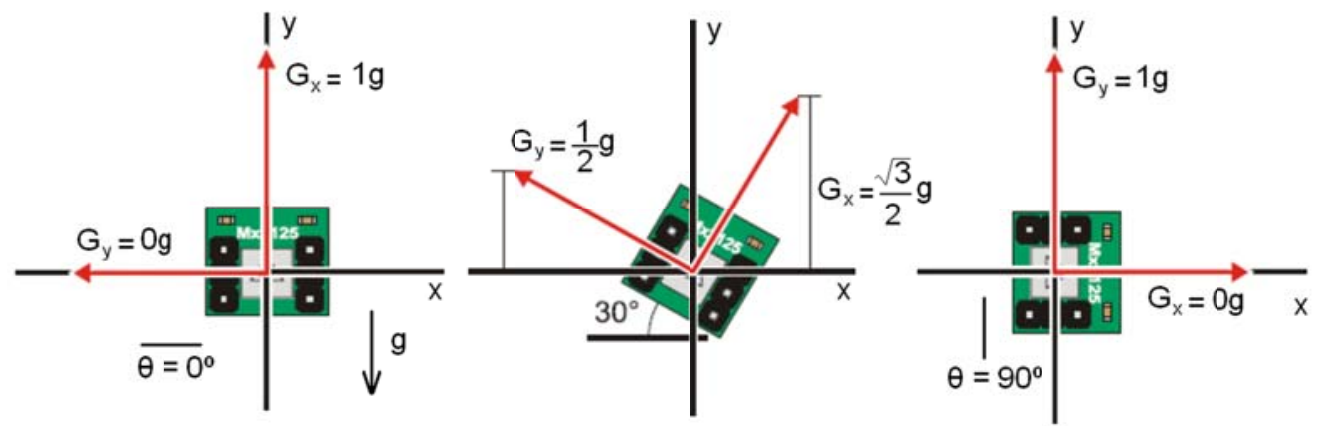

Figura 4.11 - Medida de inclinação com o MX2125 em três inclinações distintas.

No primeiro caso o acelerômetro está com inclinação de $0^{\circ}$ em relação ao plano de referência. Nota-se que no eixo y do sensor o valor de $\mathrm{G}_{\mathrm{y}}$ é nulo, dado que não há influência da força da gravidade sobre a câmera de gás nesse sentido. Já no eixo $x$ do sensor, nota-se que o valor de $G_{x}$ é dado pelo valor de $1 \mathrm{~g}$. Tal fato é verificado, pois nesse caso temos a máxima medida de aceleração estática, posto que a gravidade atua no mesmo sentido do sensor do eixo x. Cabe ressaltar que embora a força normal ao peso seja dado na unidade Newton $\left(N=\frac{K g \cdot m}{s^{2}}\right)$, os acelerômetros lançam mão de valores proporcionais a " $\mathrm{g}$ ".

No segundo caso, ângulo de $30^{\circ}$, verifica-se uma condição em que tanto o eixo x com o y do sensor sofrem influência da gravidade. Nesse sentido, obtém-se os valores de $G_{x}$ e $G_{y}$ através de operações trigonométricas, cujos resultados são os valores de $\mathrm{G}_{\mathrm{x}}=\frac{\sqrt{3}}{2} \cdot g$ e $\mathrm{G}_{\mathrm{y}}=\frac{1}{2} \cdot g$. Já o último caso é similar ao apresentado no primeiro, com a diferença dos eixos x por y e vice-versa. Nessa condição o eixo y do sensor está alinhado ao sentido da força gravitacional e sofre sua influência, tendo como resposta um valor de $\mathrm{G}_{\mathrm{y}}=1 \mathrm{~g}$, enquanto que o eixo $\mathrm{x}$, uma vez fora do sentido de sensibilização da gravidade, tem como valor $\mathrm{G}_{\mathrm{x}}=0 \mathrm{~g}$.

Como já mencionado, os acelerômetros não possuem na sua saída valores diretos de ângulo ou aceleração. No caso do MX2125 sua saída é um sinal de frequência $100 \mathrm{~Hz}$ com valores de tensão entre 3,3 a $5 \mathrm{~V}$. Sua medida de aceleração 
estática ou dinâmica é realizada conforme a variação do duty cycle ${ }^{14}$ do sinal. Através desse sinal de saída o sensor fica imune a ruídos ou interferências eletromagnéticas. Além disso, existem no mercado certos CIs e microcontroladores que desempenham a função de decodificadores de quadratura, o que permite a leitura direta de duty cycle.

Para o MX2125 existe uma relação, apresentada pela folha de dados que correlaciona o valor do duty cycle com a aceleração do sensor. Essa relação é dada pela eq.(4.2). Como o sensor possui dois eixos de medidas são apresentadas as relações do eixo x e do eixo y.

$$
G_{x}=\frac{\left(\frac{T_{\text {Ligado }}}{T_{\text {Ciclo }}}-0,5\right)}{0,125} \text { e } G_{y}=\frac{\left(\frac{T_{\text {Ligado }}}{T_{\text {Ciclo }}}-0,5\right)}{0,125}
$$

Através dessa relação, caracterizou-se um único sensor para a medida de inclinação. Essa caracterização foi realizada através da variação do ângulo de inclinação de um único sensor e das medidas do duty cycle dos eixos x e y. Foram realizadas 10 medidas com o acelerômetro em cada ângulo, sendo que o ensaio contou com medidas de $0^{\circ}$ a $360^{\circ}$ no sentido anti-horário. Para a realização dessas medidas foram observados os princípios de repetitividade, em outras palavras, foi conservado o mesmo mensurando, as medidas foram repetidas pelo mesmo experimentador utilizando os mesmos instrumentos e nas mesmas condições ambientais.

Do Apêndice F, verificou-se o levantamento de dados do eixo $\mathrm{x}$ do acelerômetro. Esse levantamento evidenciou a coleta de 10 medidas de duty cycle em cada ângulo de inclinação do sensor. Esses dados foram representados em valores de

${ }^{14}$ Duty Cycle, também conhecido como taxa de trabalho, é dado pela relação DutyCycle $=\frac{T_{\text {Ligado }}}{T_{\text {Ciclo }}}$, que pode ser representado no sinal periódico abaixo:

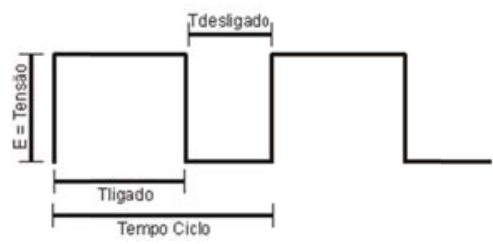


aceleração, dados pelos pontos $G_{x_{i}}$. Tal fato também pode ser visto no Apêndice G com o levantamento de dados do eixo y do acelerômetro.

A partir dessas medidas foi calculado o valor médio da aceleração estática, que estatisticamente representa o valor mais próximo do valor verdadeiro. Essa média é dada por:

$$
\overline{G_{x}}=\frac{\sum_{i=1}^{n} G_{x_{i}}}{n} \text { e } \bar{G}_{y}=\frac{\sum_{i=1}^{n} G_{y_{i}}}{n},
$$

sendo $G_{x_{1}}, G_{x_{2}}, \ldots, G_{x_{i}}, \ldots, G_{x_{10}}$, as medidas coletadas com o acelerômetro no eixo x e $G_{y_{1}}, G_{y_{2}}, \ldots, G_{y_{i}}, \ldots, G_{y_{10}}$, as medidas coletadas com o acelerômetro no eixo y. Além de $n$ representar o número de amostras coletadas, no caso 10.

Com o intuito de caracterizar as limitações do uso de acelerômetros em medidas de ADM, foram realizados alguns tratamentos estatísticos, a fim de verificar a incerteza da medida. A primeira análise foi realizada através do desvio padrão $\sigma$ do conjunto de medições, definido pela eq.(4.4).

$$
\sigma_{x}^{2}=\frac{1}{n} \sum_{i=1}^{n}\left(G_{x_{i}}-G_{x_{m v}}\right)^{2} \text { e } \sigma_{y}^{2}=\frac{1}{n} \sum_{i=1}^{n}\left(G_{y_{i}}-G_{y_{m v}}\right)^{2}
$$

Vuolo (1996) demonstra que a melhor estimativa experimental para o desvio padrão $\sigma$ do conjunto de medições é dada pela eq.(4.5). Tal estimativa foi calculada nos Apêndices F e G.

$$
\begin{aligned}
& \sigma_{x}^{2} \cong \frac{1}{n-1} \sum_{i=1}^{n}\left(G_{x_{i}}-\bar{G}_{x}\right)^{2}=\frac{1}{n-1} \sum_{i=1}^{n} G_{x_{i}}{ }^{2}-\frac{n}{n-1}{\overline{G_{x}}}^{2} \mathrm{e} \\
& \sigma_{y}^{2} \cong \frac{1}{n-1} \sum_{i=1}^{n}\left(G_{y_{i}}-\bar{G}_{y}\right)^{2}=\frac{1}{n-1} \sum_{i=1}^{n} G_{y_{i}}{ }^{2}-\frac{n}{n-1} \bar{G}_{y}{ }^{2}
\end{aligned}
$$

A melhor estimativa para o desvio padrão $\sigma_{\mathrm{m}}$ do valor médio conjunto de medições é dada pela eq.(4.6). Dessa fórmula foi obtida a base para o cálculo da incerteza estatística da medida. 


$$
\sigma_{m_{x}}=\frac{\sigma_{x}}{\sqrt{n}} \mathrm{e} \sigma_{m_{y}}=\frac{\sigma_{y}}{\sqrt{n}}
$$

Além da incerteza estatística, foi calculada a incerteza sistemática residual $\sigma_{\mathrm{r}}$ das medidas realizadas com um transferidor, cujo menor valor de leitura é de $1^{\circ}$. Como é práxis, a incerteza desse instrumento é dada pela metade da sua menor escala, no caso $0,5^{\circ}$. Esse valor acumulou nas medidas de aceleração uma incerteza sistemática de $0,0056 \mathrm{~g}$. E composto com a incerteza estatística formou a incerteza padrão $\sigma_{\mathrm{p}}$. Essa medida foi obtida, matematicamente, a partir da soma das variâncias estatísticas e da variância sistemática residual, dado pela eq.(4.7).

$$
\sigma_{p_{x}}^{2}=\sigma_{m_{x}}^{2}+\sigma_{r_{x}}^{2} \text { e } \sigma_{p_{y}}^{2}=\sigma_{m_{y}}^{2}+\sigma_{r_{y}}^{2}
$$

Tais valores também estão representados nos Apêndices F e G. Da análise dos dados foi obtido que a incerteza padrão ficou entre os valores de 0,0056g e 0,0061g tanto para o eixo x como para o eixo y. $\mathrm{O}$ que em termos estatísticos aponta para uma medida precisa, posto que há um alto grau de concordância entre os valores medidos.

Uma vez verificada a incerteza padrão da medida de aceleração estática, passou-se a calcular o ângulo medido por esse parâmetro. Para tanto foi utilizada a eq.(3.11) apresentada no capítulo 3. Nela ficou comprovada que a inclinação do sensor em relação ao plano de referência é dado pelo arco-tangente da divisão de $G_{x}$ por $\mathrm{G}_{\mathrm{y}}$. $\mathrm{O}$ Apêndice $\mathrm{H}$ apresenta os dados que foram obtidos a partir dos valores médios de $\mathrm{G}_{\mathrm{x}}$ e $\mathrm{G}_{\mathrm{y}}$ coletados para cada ângulo.

Com os valores dos ângulos medidos em comparação à referência dada pelo transferidor, esboçou-se o gráfico 4.1. Nota-se que para cada valor de referência da inclinação foi alcançado um valor muito próximo através do acelerômetro. Tal fato referendou a viabilidade da utilização de um acelerômetro como um sensor de inclinação. 


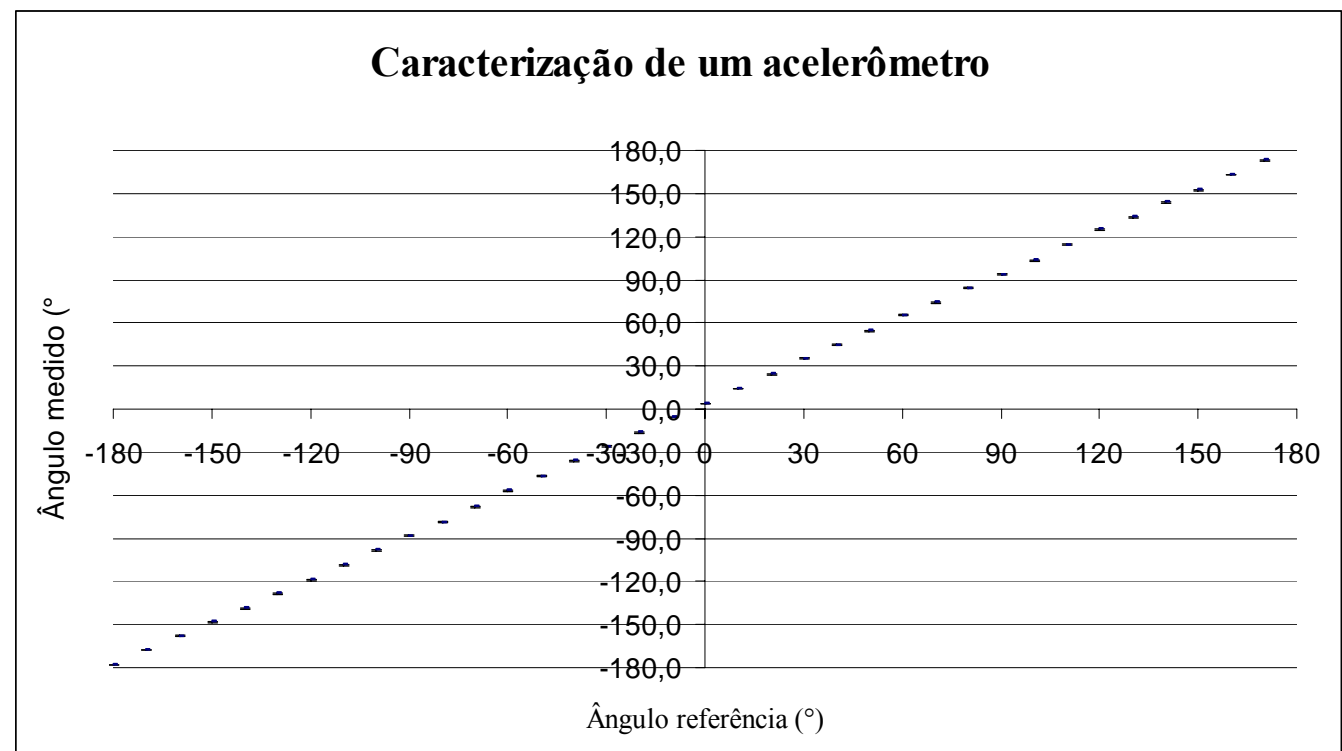

Gráfico 4.1 - Caracterização de um acelerômetro como inclinômetro.

A incerteza da medida foi calculada através da propagação de incertezas do acelerômetro no eixo $\mathrm{x}$ e no eixo y. Pois como $\theta=\theta\left(G_{x}, G_{y}\right)$, dado que $\theta=\arctan \left(\frac{G_{x}}{G_{y}}\right)$ e os valores de $\mathrm{G}_{\mathrm{x}}$ e $\mathrm{G}_{\mathrm{y}}$ são completamente independentes entre si, a incerteza padrão em $\theta$ foi obtida através da eq.(4.8) que representa a propagação de incertezas.

$$
\sigma_{\theta}^{2}=\left(\frac{\partial \theta}{\partial G_{x}}\right)^{2} \cdot \sigma_{p_{x}}^{2}+\left(\frac{\partial \theta}{\partial G_{y}}\right)^{2} \cdot \sigma_{p_{y}}^{2}
$$

Verificou-se, então, que a incerteza padrão em $\theta$ foi dada pela eq.(4.9). Esses valores estão representados tanto no Apêndice H como na barra de incertezas em y do gráfico 4.1.

$$
\sigma_{\theta}^{2}=\left(\frac{\frac{1}{G_{y}}}{1+\frac{G_{x}^{2}}{G_{y}^{2}}}\right)^{2} \cdot \sigma_{p_{x}}^{2}+\left(\frac{G_{x} \cdot \ln \left|G_{y}\right|}{1+\frac{G_{x}^{2}}{G_{y}^{2}}}\right)^{2} \cdot \sigma_{p_{y}}^{2}
$$


Numa análise quantitativa, notou-se que a incerteza padrão da medida do ângulo fica em torno de 0,12 a $0,51^{\circ}$. O que qualitativamente aponta para um método tão eficiente de medida de inclinação quanto o uso de um bom transferidor. Já a medida de erro relativo entre a referência (aqui estabelecido como valor verdadeiro) e o valor medido demonstrou que em grande parte das inclinações (22 de 35 medidas) há um erro relativo de no máximo $4 \% \mathrm{e}$, no pior caso, um erro relativo de $29 \%$.

\subsubsection{Medida de ADM com dois acelerômetros}

De posse da caracterização do acelerômetro MX2125 como inclinômetro, tornou-se possível correlacionar as leituras de dois acelerômetros, devidamente colocados em uma articulação corpórea, com as inclinações apresentadas. No caso da medida de $\mathrm{ADM}$ da flexão e extensão do cotovelo foi possível medir um ângulo de inclinação entre 20 a $180^{\circ}$. A figura 4.12 apresenta duas medidas de ADM do cotovelo. Esquematicamente, esses sensores foram alocados no braço, próximo ao ombro, e no antebraço.
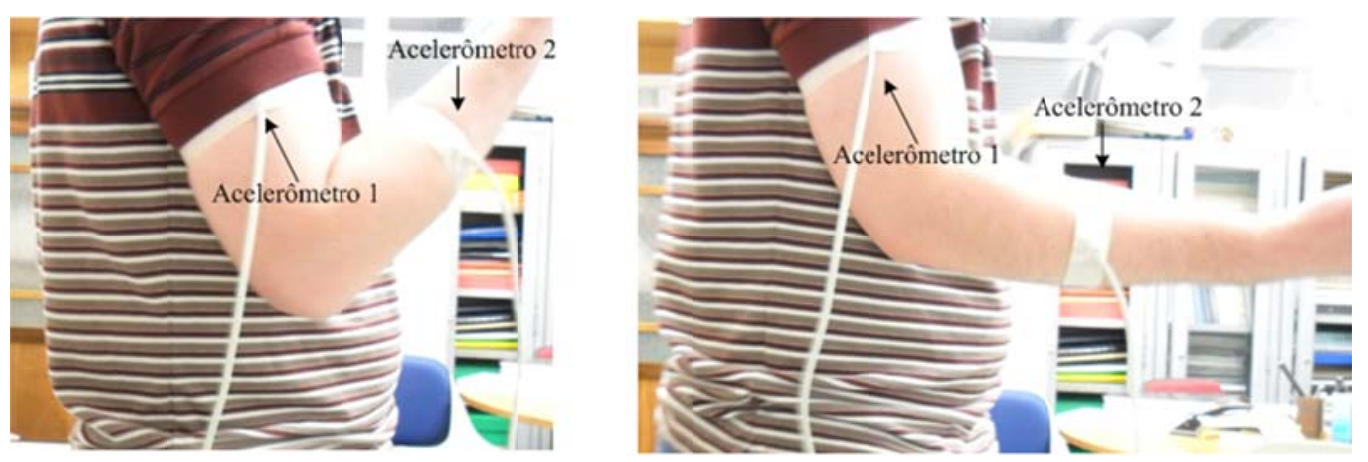

Figura 4.12 - Dois acelerômetros MX2125 alocados no braço e antebraço.

Para verificar a funcionalidade da utilização de dois acelerômetros como um eletrogoniômetro foi elaborado um ensaio variando a ADM do cotovelo nos sentidos de flexão e extensão. Esse levantamento, mostrado no Apêndice I, demonstra a 
comparação entre o ângulo de referência de um gabarito, no caso um goniômetro, e o valor de ADM calculada pelos dois acelerômetros.

Foram coletados os valores médios do duty cycle no eixo $\mathrm{x}$ e $\mathrm{y}$ dos acelerômetros 1 e 2 . A partir desses valores, calculou-se $G_{x_{1}}, G_{y_{1}}, G_{x_{2}}$ e $G_{y_{2}}$. Com esses valores foi possível medir o ângulo do primeiro acelerômetro $\left(\theta_{1}\right)$ e o ângulo do segundo acelerômetro $\left(\theta_{2}\right)$, ambos em relação ao plano de referência. A ADM foi estimada como demonstrado na eq.(3.12), através da diferença entre $\theta_{1}$ e $\theta_{2}$.

Para calcular a incerteza da medida foi aplicada a mesma propagação de erros apresentada no subcapítulo anterior. Como o valor de $A D M=\theta_{1}-\theta_{2}$, a incerteza da medida foi determinada através da eq.(4.10).

$$
\sigma_{A D M}^{2}=\sigma_{\theta_{1}}^{2}+\sigma_{\theta_{2}}^{2}
$$

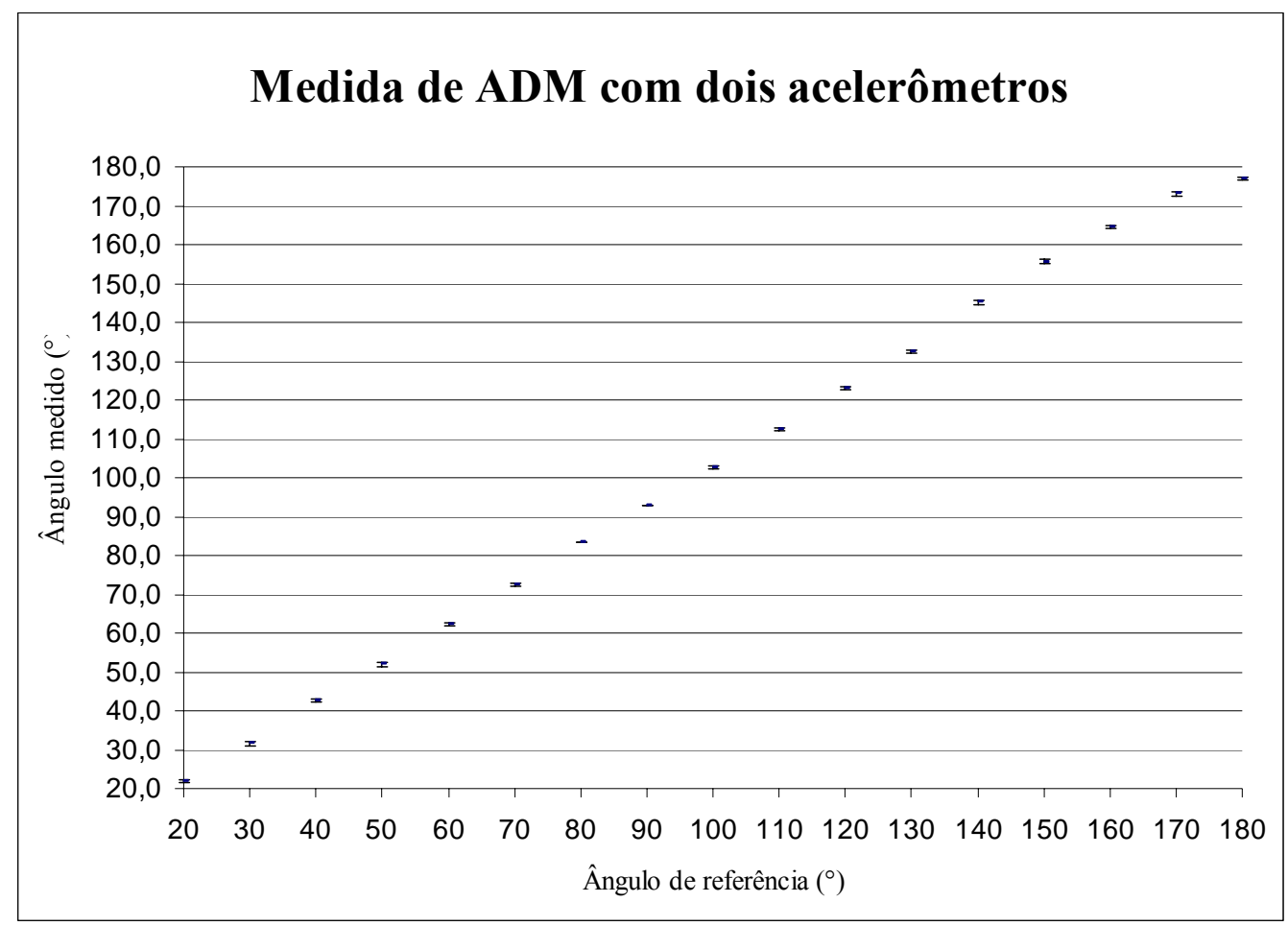

Gráfico 4.2 - Relação entre o ângulo de ADM medido pelo acelerômetro e o ângulo de referência dado pelo goniômetro. 
Tendo os valores de referência dado pelo goniômetro, o ângulo medido pelos acelerômetros e a incerteza da medida, foi possível esboçar através do gráfico 4.2 a relação entre ângulo de referência (eixo das abscissas) e o ângulo medido (eixo das ordenadas).

A incerteza máxima da medida de ADM foi de $0,7^{\circ}$, ou seja, as incertezas das medidas com cada acelerômetro se somaram (de acordo com a equação 4.10), daí um valor maior do que a medida com um único sensor. $\mathrm{O}$ erro relativo da medida de ADM com o acelerômetro ficou próximo de $4 \%$. Sendo que na caracterização do ângulo $20^{\circ}$ esse erro relativo teve seu máximo em $9 \%$.

Como já dito no capítulo 3, o principal problema dos goniômetros e dos eletrogoniômetros é sua montagem. Os erros relativos encontrados são em larga medida problemas de alocação da régua do goniômetro e do sensor. Além disso, cabe ressaltar problemas decorrentes das diferentes interpretações de posicionamento e de leitura, como, por exemplo, a paralaxe.

Alguns trabalhos, como Elveru et al. (1988), demonstram a facilidade de mensuração de variáveis articulares dos membros inferiores através de eletrogoniômetros. Os autores validaram o instrumento com erros relativos em torno de $2 \%$. Contudo cabe ressaltar que os autores utilizaram outro tipo de sensoriamento elétrico e não acelerômetros. Esses valores ficaram próximos aos valores do instrumento aqui proposto.

Outra questão apontada por Durward et al. (2001) é a velocidade de resposta do sensor. Segundo os autores o equipamento deve ter no mínimo o dobro da velocidade do movimento a ser estudado. Sobre esse aspecto o instrumento proposta não possui nenhum impeditivo, posto que a leitura é feita com os membros parados. Além disso, a resposta dos circuitos digitais utilizados está na casa da dezena de milissegundos.

Confirmada a eficiência do uso de acelerômetros como medidores de ADM, foi programado no sistema embarcado microcontrolado uma rotina que mede o duty cycle da saída dos sensores acelerômetros e através das equações 4.2, 3.13 e 3.14 consegue obter o valor de ADM automaticamente. 


\subsection{Prototipagem dos sistemas embarcados microcontrolados}

Para o desenvolvimento do módulo STIM, foi projetada uma placa de circuito impresso como visto no Apêndice K. A placa com os componentes e circuitos integrados é mostrada na figura 4.13. Nessa placa além das entradas para os acelerômetros, verificam-se a interface USB e o microcontrolador AT90USB162. Nesse microprocessador foram implementados os protocolos IEEE1451.0 e IEEE1451.2.

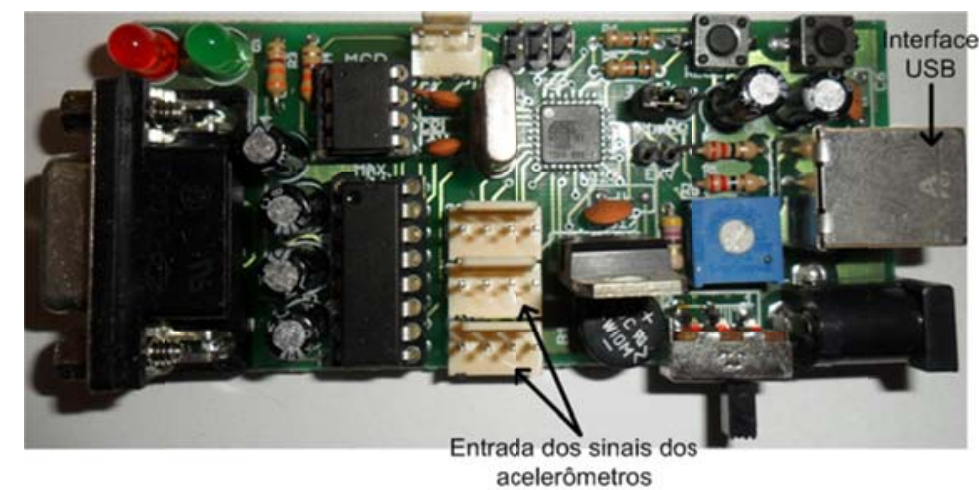

Figura 4.13 - Sistema embarcado microcontrolado para desenvolvimento do STIM.

Para a comunicação via USB foi utilizado o framework LUFA (Lightweight USB Framework for $A V R s)^{15}$. A partir dessa biblioteca foi programada uma porta serial virtual utilizando a classe $C D C$. Nessa configuração os dados ficam disponíveis através de uma porta de comunicação "COM" no sistema operacional Windows ou um dispositivo “/dev/ttyS" no sistema operacional Linux. Tal fato permite uma comunicação de dados em alta velocidade entre o módulo STIM e o NCAP instalado em um computador.

Para a construção do nó WTIM, além do circuito de condicionamento de biopotenciais foi realizado o projeto e a prototipagem de um sistema embarcado microcontrolado utilizando o microcontrolador ATmega8535 e o módulo Wifi Wifly

\footnotetext{
${ }^{15}$ Disponível em: http://www.fourwalledcubicle.com/LUFA.php. Acesso em: 10 de agosto de 2010.
} 
GSX, como descrito no capítulo 3. A figura 4.14 apresenta a placa que foi confeccionada e utilizada para realizar a aquisição de sinais do circuito condicionador e a posterior transmissão via Wifi dos dados coletados de acordo com os protocolos IEEE1451.0 e IEEE1451.5.

A figura 4.14 apresenta um conector coaxial conectado a uma entrada do conversor analógico-digital do microcontrolador. Essa entrada permite amostrar tanto a parte negativa como a positiva do sinal de ECG. Esse conector coaxial foi conectado diretamente na saída do circuito da figura 4.8. O projeto do circuito está apresentado no Apêndice J.

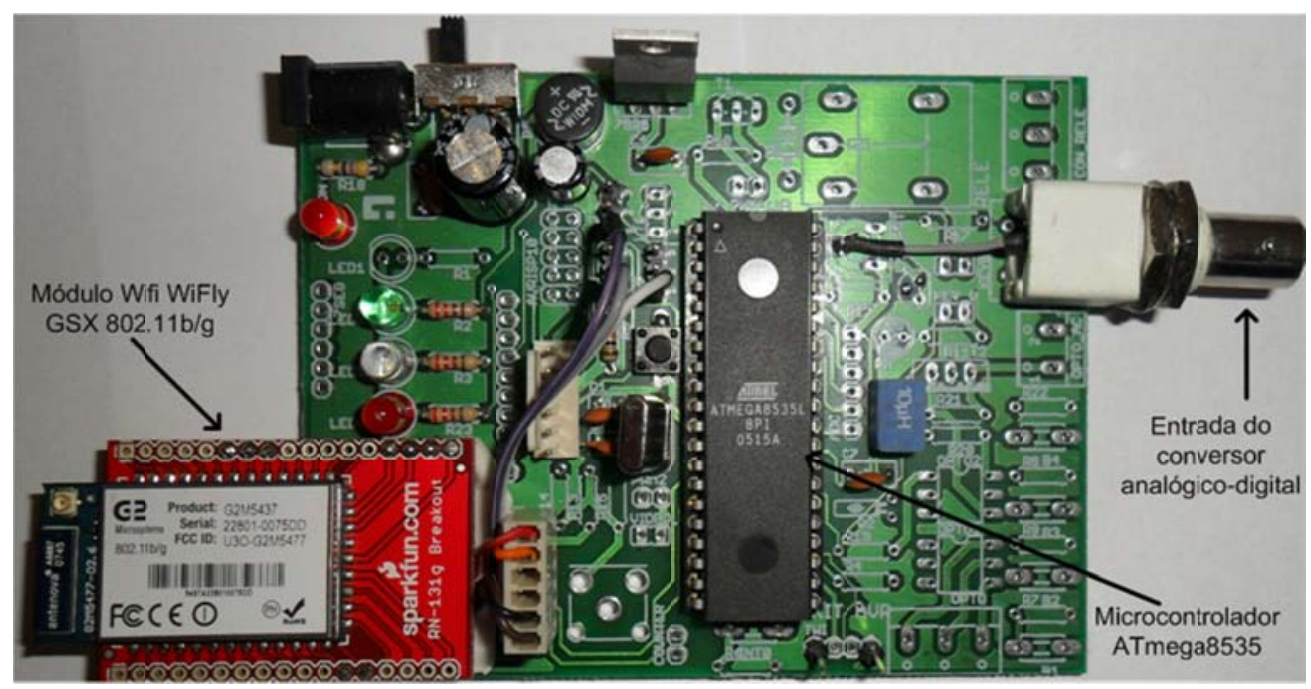

Figura 4.14 - Sistema embarcado microcontrolado para desenvolvimento do WTIM.

O microcontrolador teve a função de amostrar os sinais de ECG e através da contagem de tempo entre dois pulsos $\mathrm{R}$ realizou uma aproximação da frequência cardíaca do paciente. Sendo que a medida da quantidade de batimentos por minuto foi feita dividindo o valor 60 pelo tempo medido.

Os dados coletados foram transmitidos conforme solicitados pelo NCAP através do módulo Wifly. Esse, como já mencionado no capítulo 3, possui baixo consumo e se comunica com o microcontrolador através de uma conexão serial $U A R T$ de três fios ( $T X, R X$ e GND). 
Através desse módulo foi realizada uma conexão $A d$ hoc entre o nó WTIM e o computador. A configuração foi feita através de uma sintaxe simples, que permite configurar as informações de SSID (Service Set Identifier), no caso IEEE1451; canal, 1; endereço de $I P$, no caso foi escolhido um valor fixo de 192.168.160.10; uma máscara de rede, 255.255.255.0; e um IP de gateway, 192.168.160.1, que indica o endereço do computador (NCAP).

\subsection{Desempenho dos nós IEEE1451}

Com os hardwares dos TIMs e do NCAP definidos, foram verificadas as funcionalidades do IEEE1451 e sua dinâmica. Para isso são apresentados nos subcapítulos seguintes os resultados do desenvolvimento do $N C A P$, cujo objetivo é o de apresentar as informações coletadas pelos nós sensores e disponibilizar através de uma rede de controle, no caso a Internet. Além disso, exibe-se o desenvolvimento da programação do lado dos TIMs (WTIM e STIM).

\subsubsection{Desenvolvimento do NCAP}

O software $N C A P$ foi escrito na linguagem Java ${ }^{16}$ e executado em uma máquina virtual no sistema operacional Windows $X p$. Seus objetos e métodos tem com base o modelo da figura 4.15. Nesse diagrama estão os resultados obtidos no desenvolvimento do software do NCAP. No modelo ficam evidentes duas partes fundamentais do processador de aplicação: os módulos de comunicação IEEE1451.2 e IEEE1451.5, e a estrutura do IEEE1451.0, que permite decodificar as informações transmitidas pelos TIMs.

Para o módulo de comunicação serial do $N C A P$ foi utilizada a biblioteca CommJar, parte integrante da Java Communications API. Através dos comandos dessa biblioteca foi possível configurar e acessar todo tipo de dado transmitido

\footnotetext{
16 A escolha pela linguagem orientada a objeto Java se deu devido a sua capacidade em ser multiplataforma, ou seja, a possibilidade de portabilidade. O que permitiria ao $N C A P$ em questão ser instalado em diferentes sistemas operacionais e plataformas computacionais.
} 
serialmente. Para o módulo de comunicação IEEE1451.5 e sua comunicação $T C P / I P$ iniciada após a conexão Wifi, foram aplicados os métodos e as funções disponíveis no Java Sockets. Através desses, foi possível estabelecer um comunicação clienteservidor entre o NCAP e o WTIM, no qual este era cliente daquele.

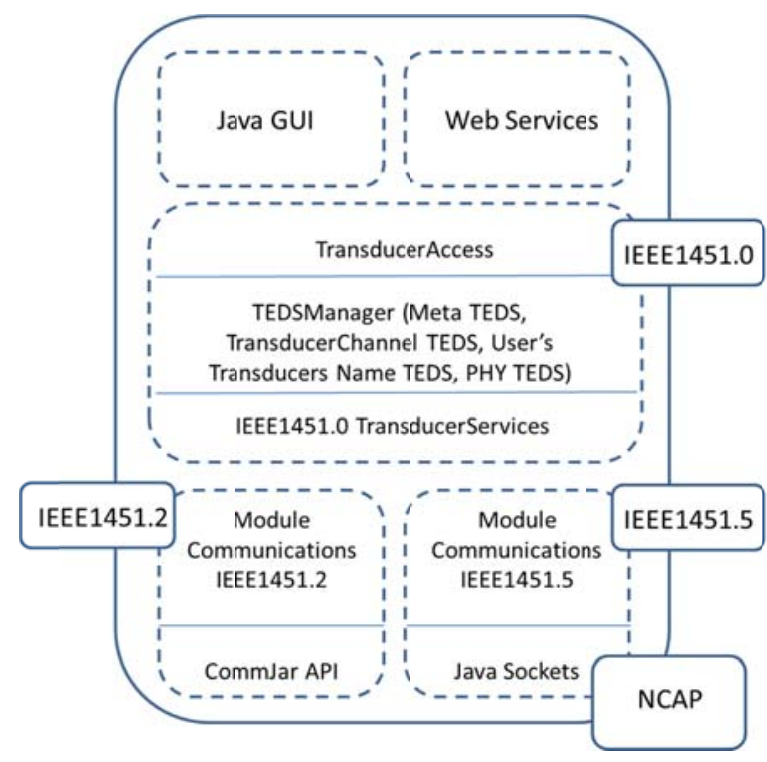

Figura 4.15 - Modelo do NCAP.

\section{Rede de Sensores para Eng. Biomédica utilizando o protocolo IEEE1451 $\square \square$} Sair Sobre

Rede de Sensores para Eng. Biomédica utilizando o protocolo IEEE1451

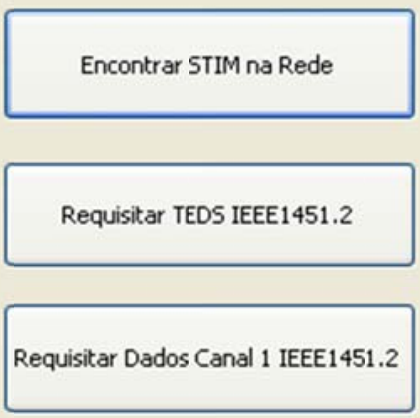

Encontrar WTIM na Rede

Requisitar TEDS IEEE1451.5

Requisitar Dados Canal 1 IEEE1451.5

Figura 4.16 - Interface do software NCAP desenvolvido. 
A interface gráfica do $N C A P$ foi desenvolvida utilizando os recursos do Swing GUI Designer do Eclipse, como mostrado na figura 4.16. Nessa interface ficam disponíveis os recursos: de recebimento de informações do STIM e WTIM na rede; de requisição dos TEDS do STIM (IEEE1451.2) e dos TEDS do WTIM (IEEE1451.5); e de recepção e apresentação dos dados coletados pelos sensores dos nós STIM e WTIM.

\subsubsection{Desenvolvimento dos nós IEEE1451.2}

O desenvolvimento do nó IEEE1451.2 contou com a construção e configuração dos TEDS, conforme apresentado no subcapítulo 3.6. A programação da norma foi desenvolvida utilizando a linguagem ANSI $\mathrm{C}$ associada a classes da linguagem orientada a objetos $\mathrm{C}++$. Como resultado do desenvolvimento do nó STIM foi obtido o modelo da figura 4.17.

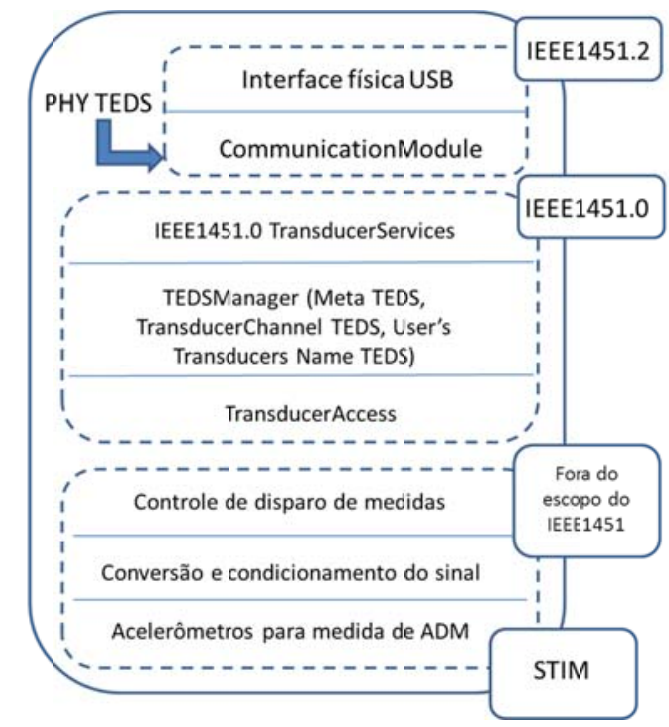

Figura 4.17 - Modelo do nó IEEE1451.2.

Para realizar os testes do nó IEEE1451.2 com a interface NCAP foram seguidos os passos de funcionamento, tal como apresentado no diagrama sequencial UML (Unified Modeling Language) da figura 4.18. Como já mencionado, o nó STIM 
sempre responde a uma requisição do $N C A P$, seja de dados, seja de TEDS. A única mensagem que é feita independente do NCAP é a Tim Initiated Message.

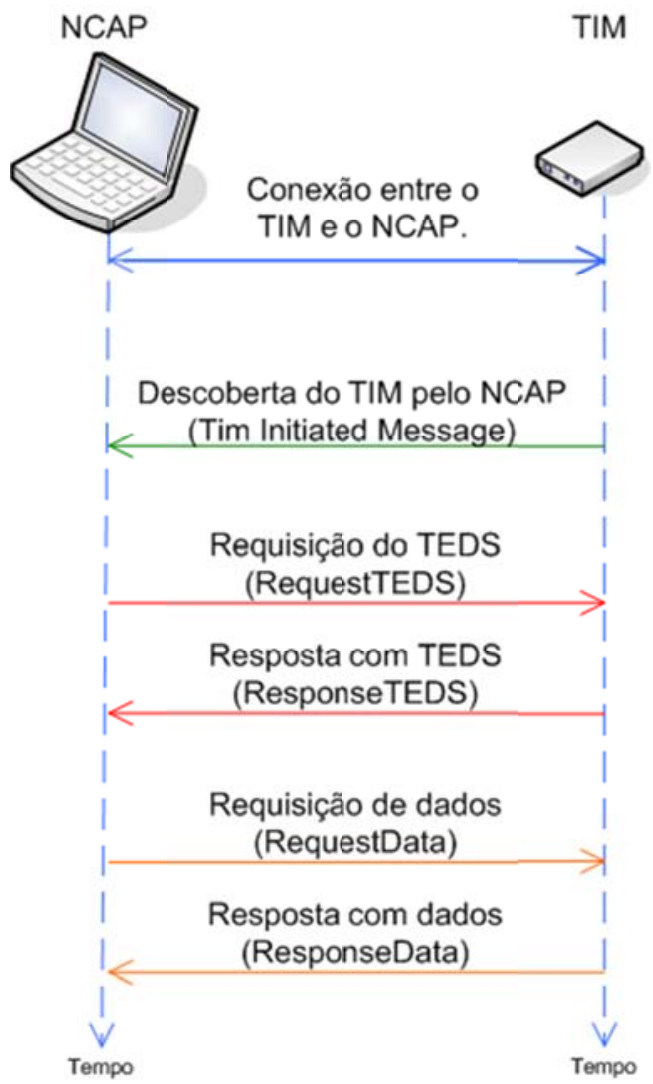

Figura 4.18 - Diagrama sequencial de conexão entre o $T I M$ e o $N C A P$.

Através da primeira mensagem enviada pelo $S T I M$, o $N C A P$ já é capaz de encontrar alguns dados a respeito desse nó sensor. Nesse registro o NCAP armazena os valores dos TEDS e os interpreta. Os valores dos TEDS, para o STIM, são aqueles encontrados na última coluna das tabelas 3.4, 3.6, 3.8 e 3.10. Esses valores em formato hexadecimal foram transmitidos e o $N C A P$ foi responsável em verificar cada campo, decodificar seu valor e registrar essa informação localmente. Após esse passo os dados ficaram disponíveis para exibição na interface Java ou através do Web Service.

No botão "Encontrar STIM na Rede", são apresentadas para o usuário as informações do PHY TEDS enviadas pelo STIM. No caso do teste com o nó sensor 
IEEE1451.2 foram registrados os dados: "Número do módulo de comunicação NCAP" (TIM ID): 1; "Identificação do módulo de comunicação NCAP" (Comm Module ID): 119; "Número do módulo de comunicação STIM" (Comm Module Type): 1; "Identificação do módulo de comunicação STIM" (ID of Channel): 301; "Número de Canais do STIM" (Channel Numbers of TIM): 1; "Identificação do Canal 1 e nome" (ID of Channel e Name of Channel): 1 IEEE1451.2 Accelerometer_Sensor. Esses valores estão apresentados na figura 4.19.

\section{Encontrar STIM na Rede}

\section{Encontrar dados do STIM}

Número do módulo de comunicação NCAP:

Identificação do módulo de comunicação NCAP:

Número do módulo de comunicação STIM:

Identificação do módulo de comunicação STIM:

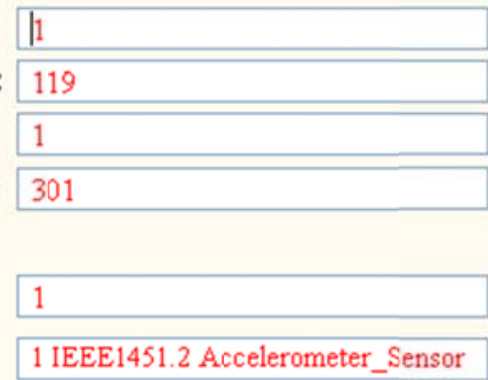

Número de Canais do STIM:

Identificação do Canal 1 e nome:

1 IEEE1451.2 Accelerometer_Sensor

Figura 4.19 - Acesso às informações do STIM.

$\mathrm{Na}$ figura 4.20 são apresentadas as informações que foram requisitadas pelo usuário ao selecionar a opção "Requisitar TEDS IEEE1451.2". Como resposta a essa requisição foram transmitidos pelo STIM alguns valores configurados no Meta TEDS, TransducerChannel TEDS e no User's Transducer Name TEDS. Dentre os dados estão: "Identificação do fabricante" (Manufacturer ID): gSIM; "Número do canal" (Number of Channel): 1; "Versão dos TEDS" (Version of TEDS): v1; e "Número de série" (Serial Number): 125. 


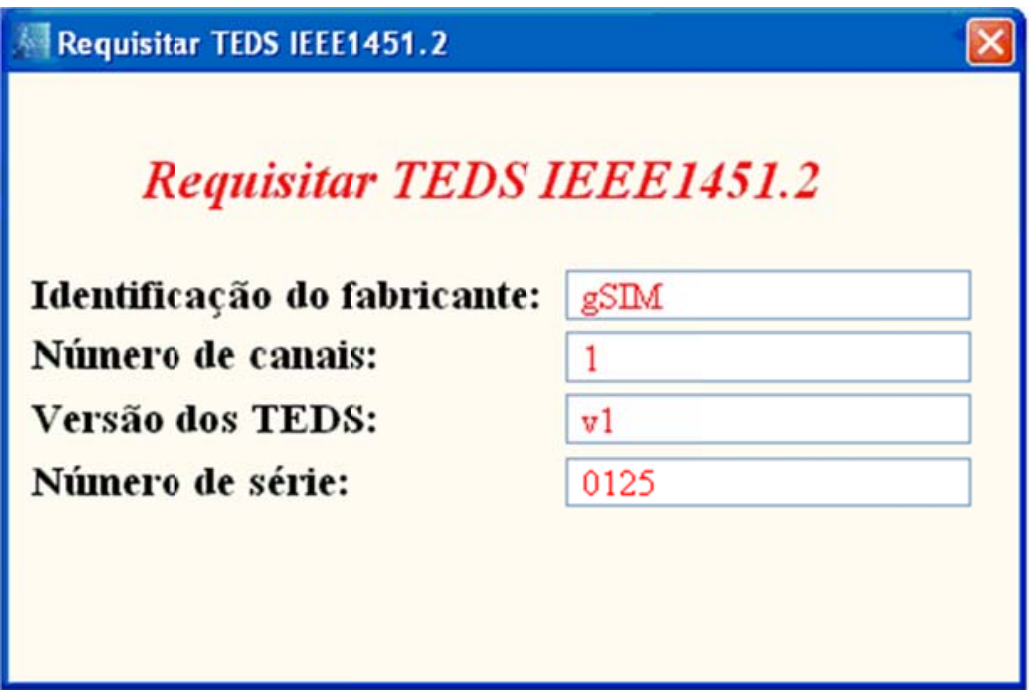

Figura 4.20 - Acesso às informações dos TEDS do STIM.

Por último foram requisitados os dados amostrados, processados, agregados e transmitidos através da interface USB para o $N C A P$, conforme apresentado na figura 4.21. Na operação de teste foi obtido o valor de ADM de $42^{\circ}$.

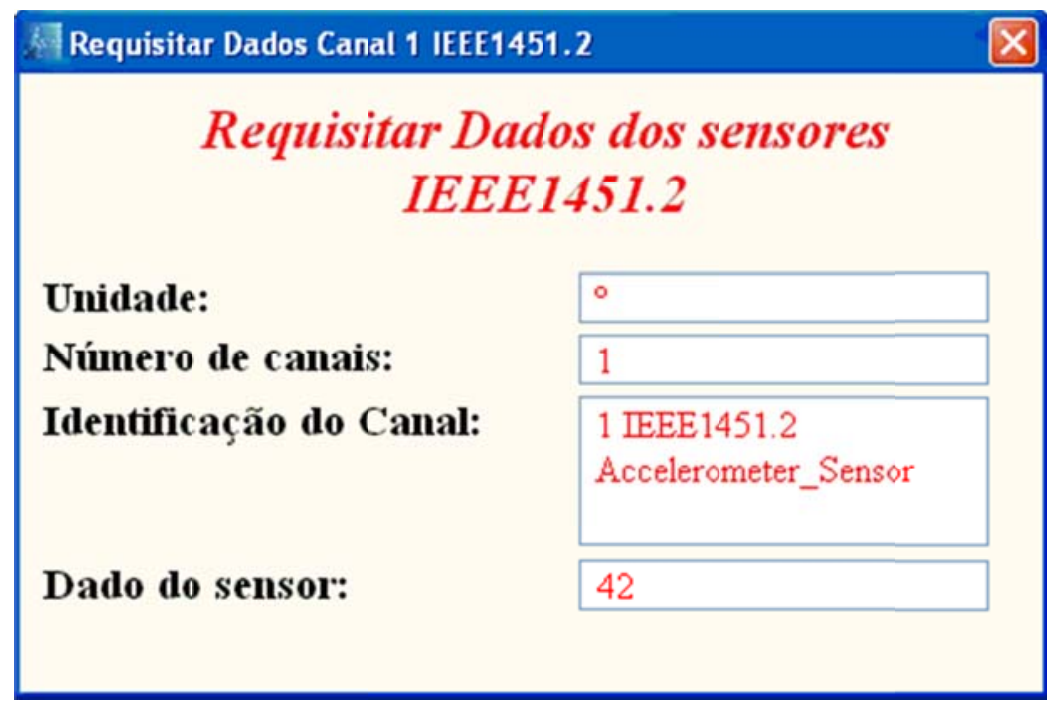

Figura 4.21 - Disponibilização dos dados coletados no sensor do STIM. 


\subsubsection{Desenvolvimento dos nós IEEE1451.5}

Similar ao apresentado na seção anterior, para o nó IEEE1451.5 foram utilizadas as configurações dos TEDS conforme apresentado no subcapítulo 3.7. A programação da norma também foi feita utilizando a linguagem ANSI C associada à linguagem $\mathrm{C}++. \mathrm{O}$ modelo desenvolvido do nó IEEE1451.5 está apresentado na figura 4.22 .

Os testes dinâmicos com o nó IEEE1451.5 e a interface $N C A P$ tomaram como referência o diagrama sequencial $U M L$ da figura 4.18, sendo que a conexão Wifi foi estabelecida entre o modulo Wifly GSX e uma placa Wifi interna a um netbook. Para essa conexão não foram utilizados os protocolos de encriptação via rádio ${ }^{17}$. $\mathrm{O}$ nó WTIM estabeleceu inicialmente a transmissão da Tim Initiated Message, que é responsável por enviar os dados do PHY TEDS. Após esse envio o WTIM passou somente a responder requisições do $N C A P$.

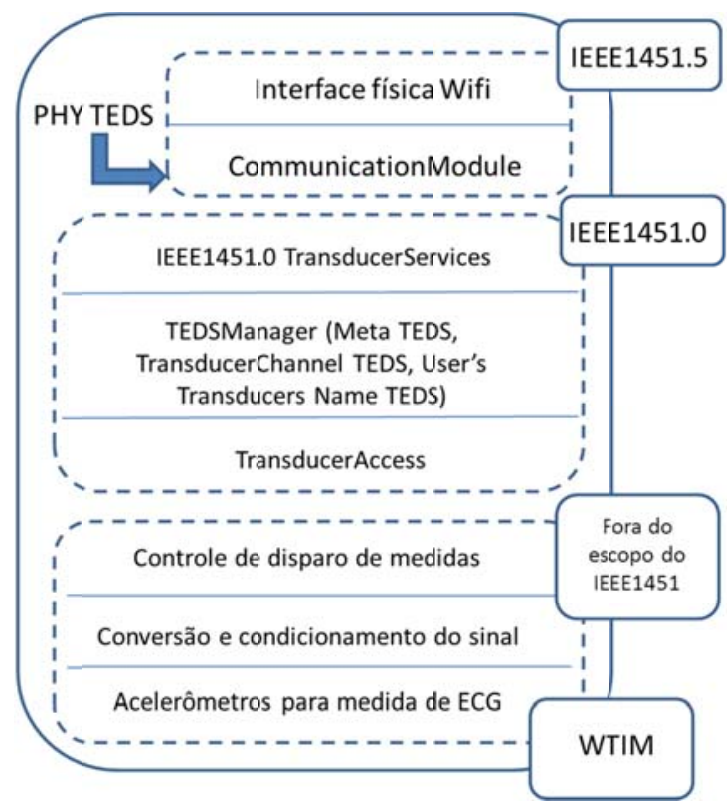

Figura 4.22 - Modelo do nó IEEE1451.5.

\footnotetext{
${ }^{17}$ Possíveis encriptações para transmissão Wifi são: WPA (Wifi Protected Access), WPA2, WEP (Wifi Equivalent Privacy). Através desses padrões de segurança as informações podem ser transmitidas com maior segurança pela rede sem fio.
} 


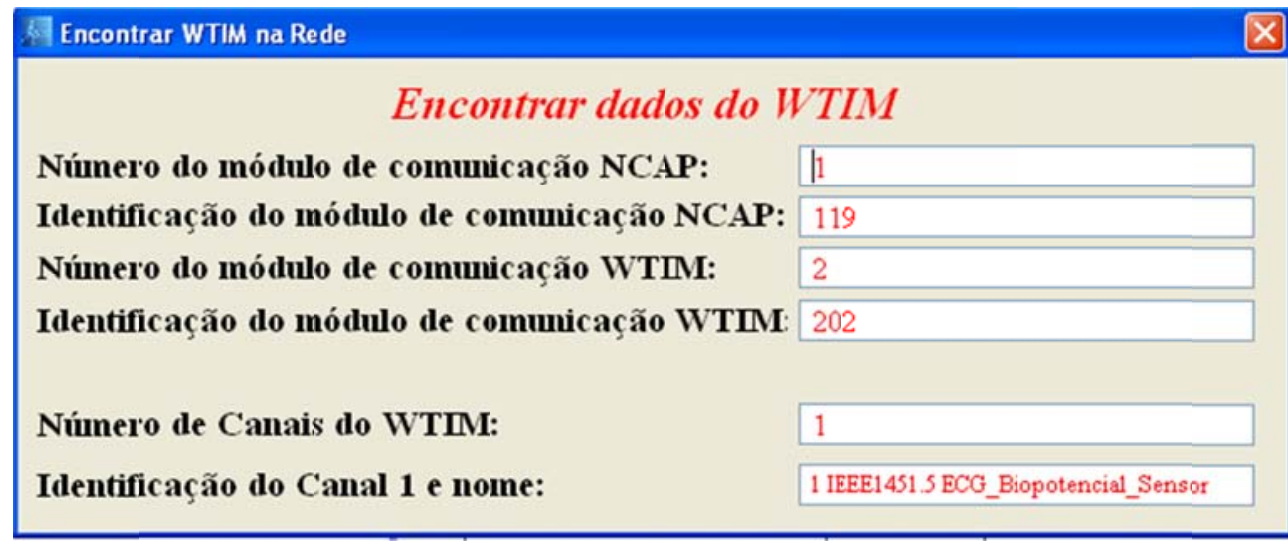

Figura 4.23 - Acesso às informações do WTIM.

Com o envio dos valores do PHY TEDS, o NCAP foi capaz de "Encontrar o WTIM na Rede". No teste com o nó IEEE1451 foram obtidos, conforme a figura 4.23, os seguintes dados: "Número do módulo de comunicação NCAP" (TIM ID): 1; "Identificação do módulo de comunicação NCAP" (Comm Module ID): 119; "Número do módulo de comunicação WTIM" (Comm Module Type): 2; "Identificação do módulo de comunicação WTIM" (ID of Channel): 202; "Número de Canais do WTIM" (Channel Numbers of TIM): 1; "Identificação do Canal 1 e nome" (ID of Channel $e$ Name of Channel): 1 IEEE1451.5 ECG_Biopotencial_Sensor.

No NCAP foi possível, após a recepção dos PHY TEDS do IEEE1451.5, requisitar parte dos dados dos Meta TEDS, TransducerChannel TEDS e do User's Transducer Name TEDS ao se selecionar a opção "Requisitar TEDS IEEE1451.5". $\mathrm{Na}$ figura 4.24 são apresentadas as informações transmitidas do WTIM em resposta a pedido do NCAP: "Identificação do fabricante" (Manufacturer ID): gSIM; "Número do canal" (Number of Channel): 1; "Versão dos TEDS" (Version of TEDS): v1; e "Número de série" (Serial Number): 125.

Como dito anteriormente, os dados dos TEDS, em específico para o WTIM, são aqueles encontrados na última coluna das tabelas 3.5, 3.7, 3.9 e 3.11. Esses valores em hexadecimal transmitidos ao $N C A P$ foram decodificados e interpretados. Após isso, exibidos na interface local através do aplicativo Java, ou requisitados remotamente pelo Web Service. 


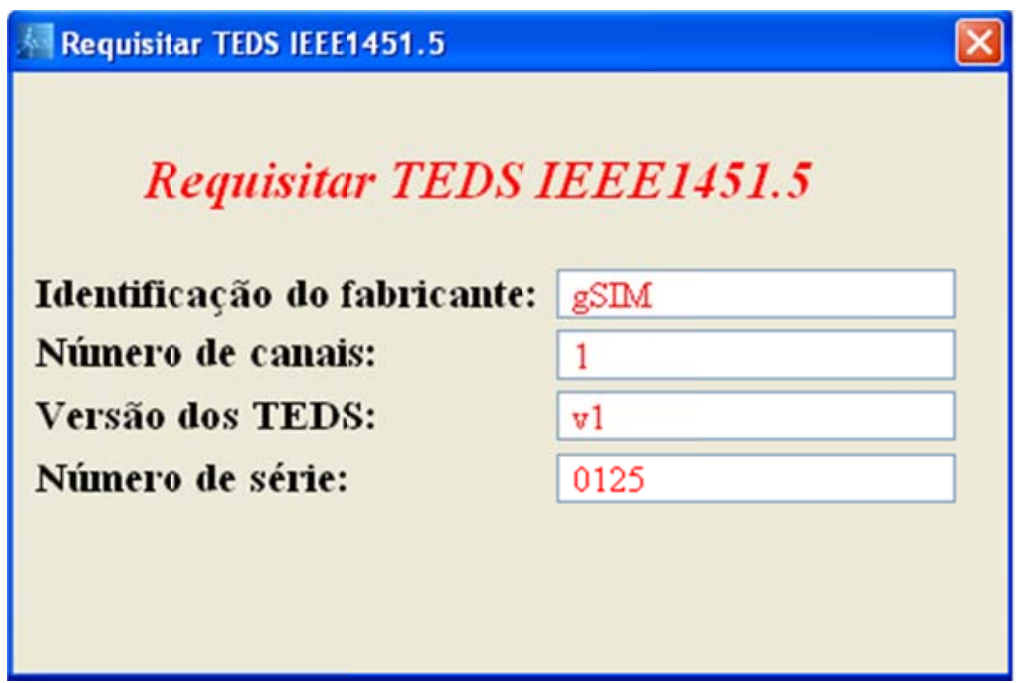

Figura 4.24 - Acesso às informações dos TEDS do WTIM.

Por último os dados mensurados pelos sensores foram recebidos pelo $N C A P$, quando solicitados através do pedido "Requisitar Dados do Canal 1 IEEE1451.5". A figura 4.25 apresenta o valor de frequência cardíaca igual a 72 batimentos por minuto.

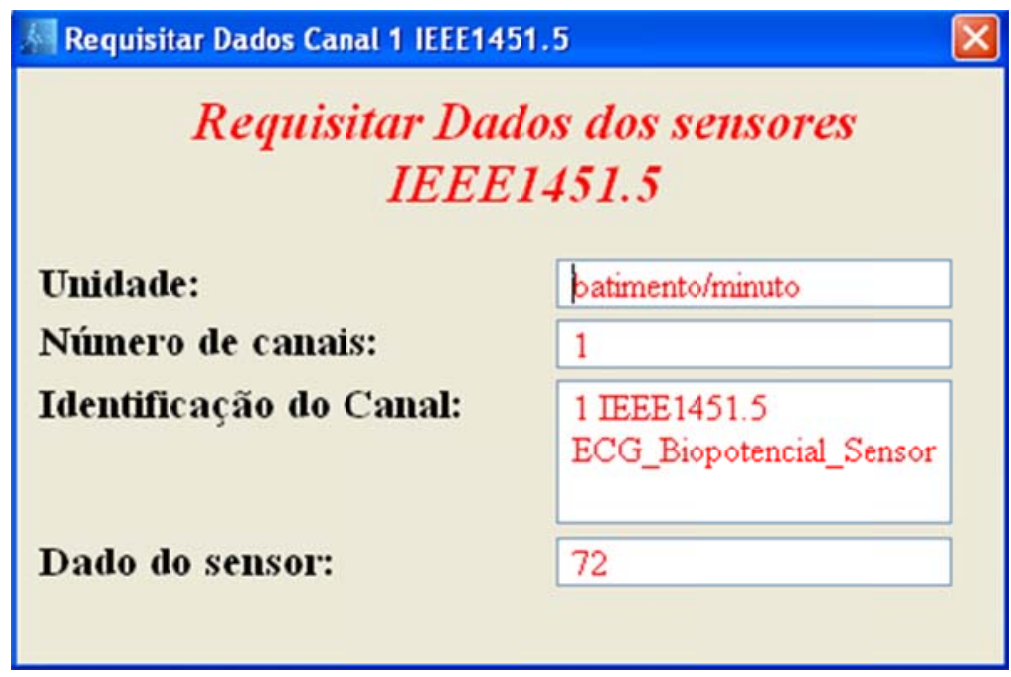

Figura 4.25 - Disponibilização dos dados coletados no sensor do WTIM. 


\subsection{Interface com Web Services}

O acesso remoto dos dados coletados pelos sensores e o nome de cada sensor foi feita através de um Web Service, escrito a partir do framework Axis2 (GOMES, 2010) (ALBINADER NETO, 2006).

Esse Web Service possuiu como Provedor de Serviços o programa NCAP desenvolvido em Java, como já mencionado anteriormente. Para o Consumidor de Serviços foi utilizado o padrão fornecido pelo ambiente Eclipse para clientes J2EE. O Registro de Serviços foi realizado localmente, o que permitiu a comunicação entre o cliente e o provedor Web Service sem intermediações.

$\mathrm{Na}$ figura 4.26 é apresentado o WSDL do Web Service. Como visto no capítulo 2, o WSDL descreve o Web Service, especificando suas operações e definindo o formato de entrada e saída de cada operação. Os dois recursos "STIM" e “WTIM” descritos no WSDL disponibilizam como resposta (STIM/WTIM Response) o nome do sensor e o valor do dado coletado.

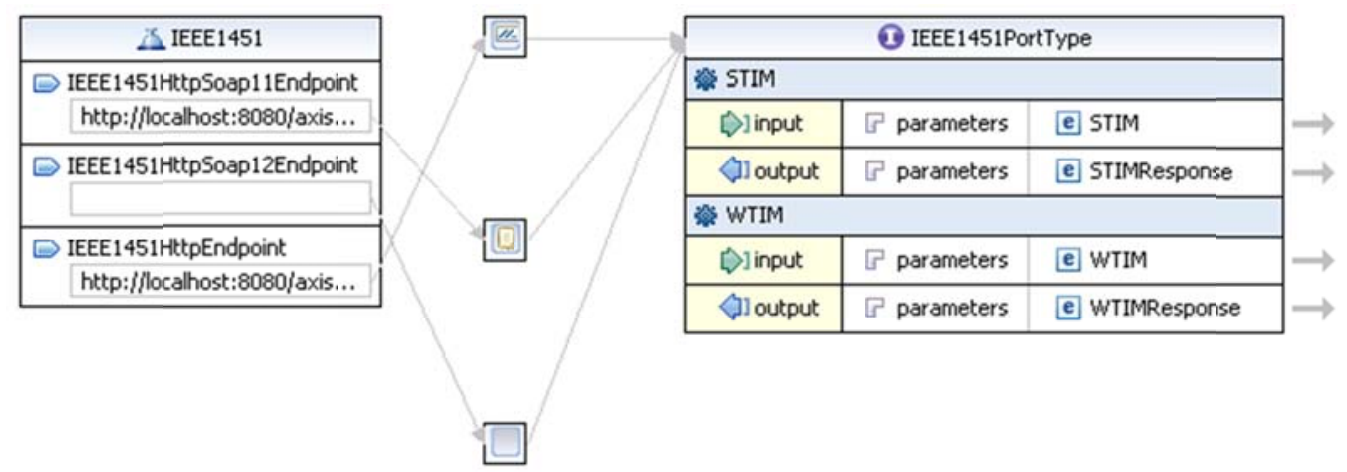

Figura 4.26 - Descrição WSDL do Web Service desenvolvido.

A partir da descrição dos serviços do Web Service, o software de acesso foi alocado dentro do servidor Apache Tomcat 6.0. Esse servidor permitiu que as informações fossem acessadas pela rede. No caso dos testes desenvolvidos o acesso foi feito localmente através da $U R L$ : http://localhost:8080/axis2/services/IEEE1451. 
Primeiramente foi realizado o teste para requisitar as informações do STIM. Após a conexão com o servidor, foi requisitada a informação do nome do STIM e do dado coletado pelo sensor. $\mathrm{O}$ arquivo $X M L$ de resposta do servidor está descrito abaixo:

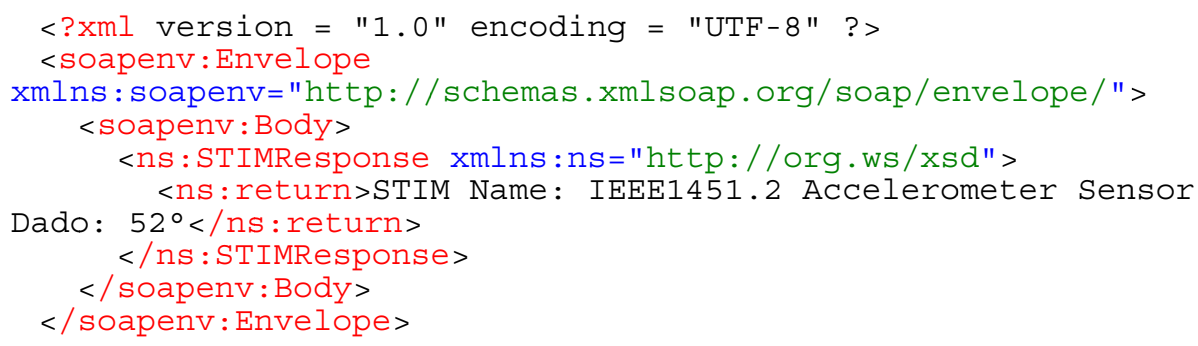

Nesse arquivo é apresentado no cabeçalho "ns:return", o valor referente ao nome do STIM, no caso "IEEE1451.2 Accelerometer_Sensor", além do dado coletado no momento do funcionamento do Web Service, ADM de "52". Em específico, a figura 4.27 apresenta no campo "STIMResponse" esse mesmo valor, que corresponde a decodificação do arquivo $X M L$ pelo cliente.

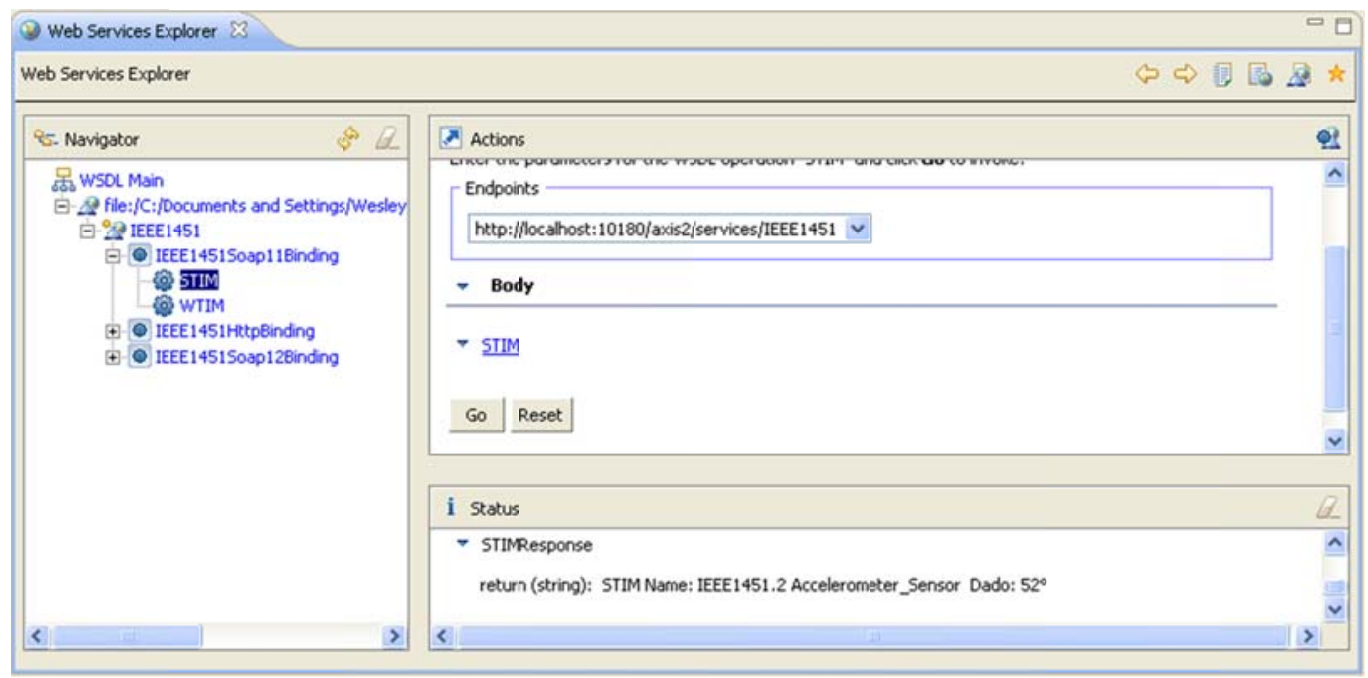

Figura 4.27 - Resposta do NCAP à requisição de dados do STIM. 
Da mesma maneira apresentada no STIM, para a requisição do nome e dado do WTIM, o Web Service respondeu com o arquivo $X M L$ descrito abaixo:

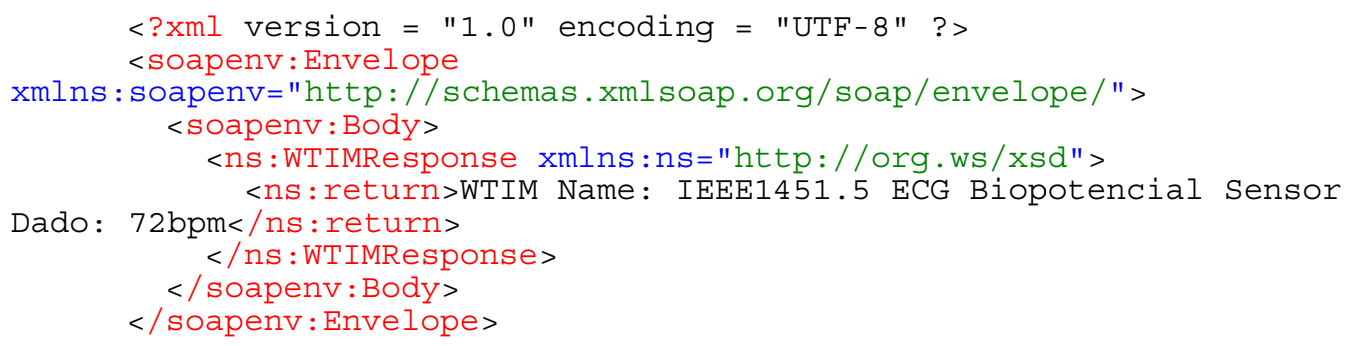

No arquivo é apresentado no cabeçalho "ns:return", o valor referente ao nome do WTIM, no caso “IEEE1451.5 ECG_Biopotencial_Sensor”, além do dado coletado no momento do funcionamento do Web Service, frequência cardíaca de "72bpm". Em específico, a figura 4.28 apresenta no campo "WTIMResponse" a decodificação do arquivo $X M L$ pelo cliente.

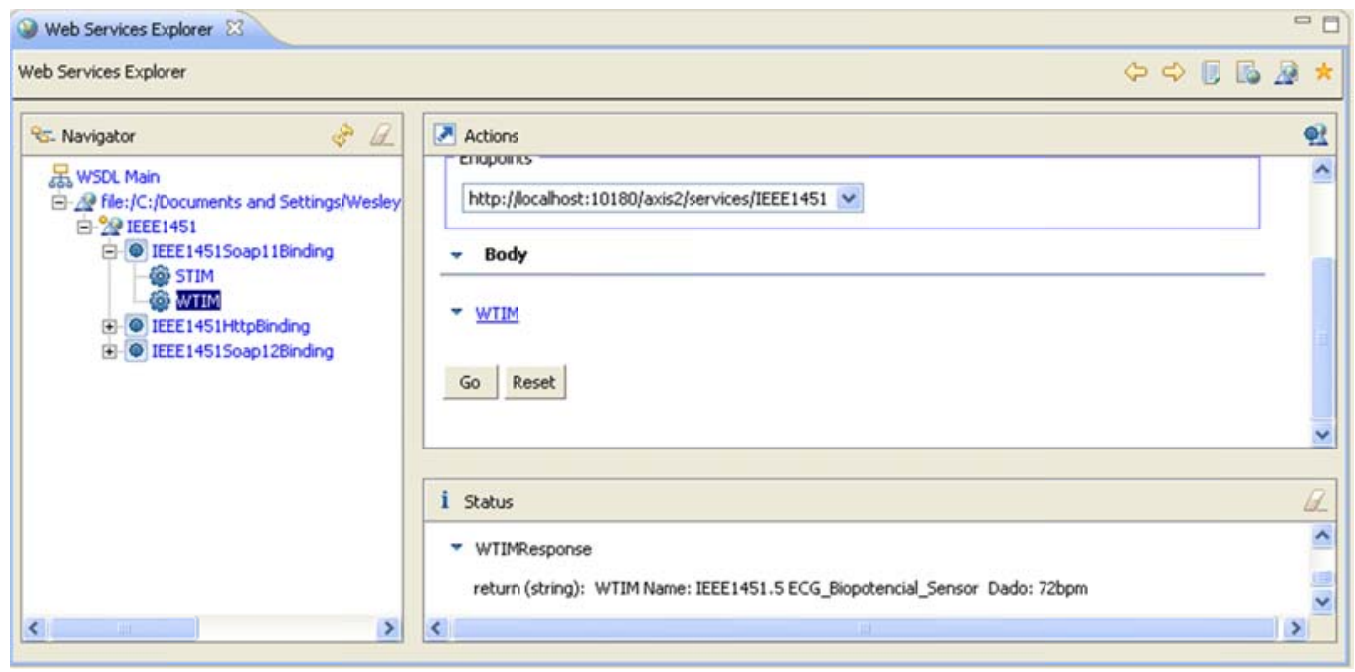

Figura 4.28 - Resposta do $N C A P$ à requisição de dados do WTIM.

Diferente de outros trabalhos (SADOK; LISCANO, 2005) (SONG; LEE, 2008c) no qual o Web Service era capaz de requisitar informações diretamente do nó TIM, no trabalho em questão, o Web Service somente requisita os dados que já estão 
registrados no NCAP. Para tanto, o serviço Web Service disponibilizou uma requisição para envio das informações do STIM e do WTIM.

\subsection{Validação do WTIM com o framework NIST}

Para verificar a validade do desenvolvimento do WTIM e do NCAP desenvolvido foi utilizado o framework de Song e Lee (2006). Esse software (ver figura 4.29) serviu de parâmetro para validação do desenvolvimento do protocolo IEEE1451.0 e IEEE1451.5 no sistema embarcado Wifi.

Algumas modificações pontuais foram realizadas para permitir a correta interface entre o WTIM e o NCAP. Visto que originalmente o framework NIST tinha como modelo de WTIM um computador conectado serialmente a um sensor normatizado no padrão IEEE1451. Dessa maneira, tanto o NCAP como o WTIM foram programados em linguagem Java e instalados em computadores separados. Esses, por sua vez, conectavam-se pela interface Wifi e estabeleciam a dinâmica do IEEE1451.

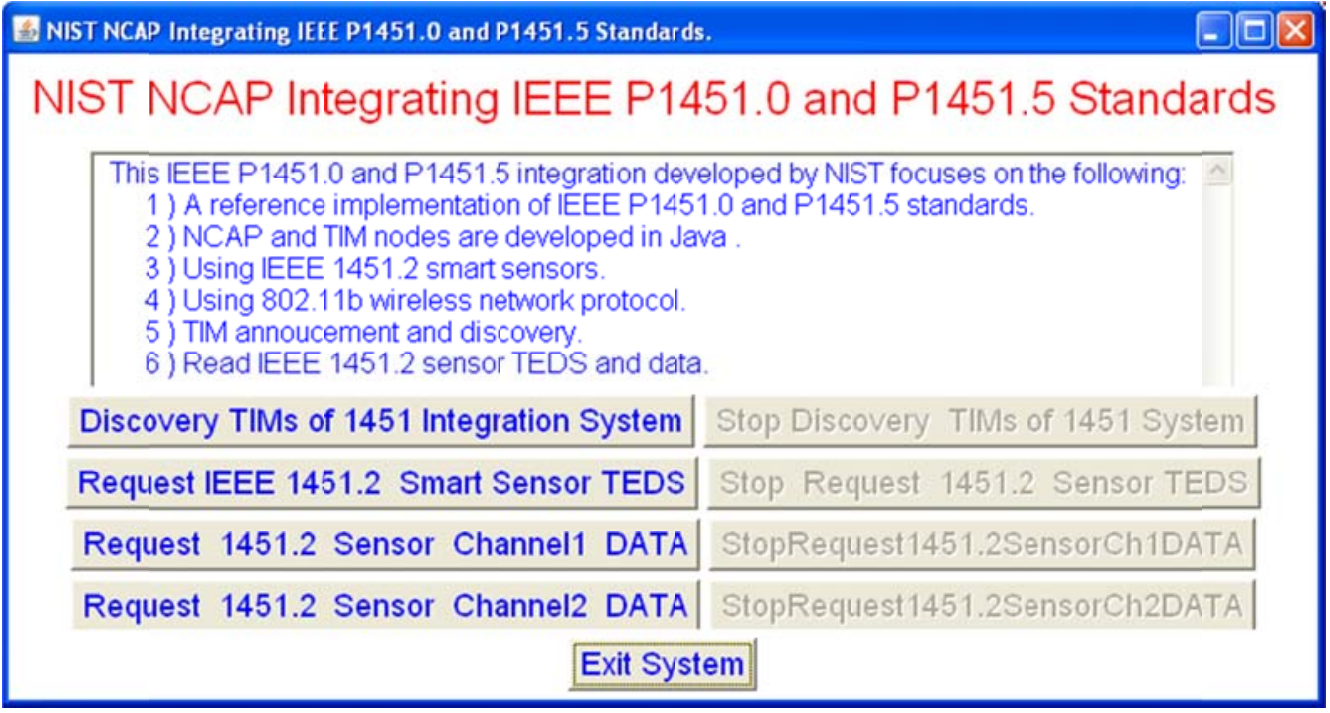

Figura 4.29 - Interface inicial do framework NIST IEEE1451.0 e IEEE1451.5. 
No caso da rede de sensores biomédicos, o WTIM é um sistema microcontrolado e não um computador. Essas modificações não mudaram o mecanismo de conexão entre o WTIM e o NCAP, que para o framework NIST é descrito em cinco etapas.

Na primeira etapa é estabelecida a identificação dos módulos NCAP e WTIM para eles próprios, ou seja, ao iniciar cada módulo é obtida a informação gravada em uma memória não volátil e integrada ao software de controle de comunicação.

A segunda etapa se reduz a transmissão de um anúncio (Tim Initiated Message) do módulo WTIM ao NCAP, como apresentado na figura 4.30. Nessa fase foram transmitidas as informações pertinentes valores do $P H Y$ TEDS, que contemplam os valores: TIM Identification, Comm Module ID, Comm Module Type, Comm Module Techonology ID, Comm Module Name, Comm Module Object, IP Address e Port. No anúncio também foram fornecidos os valores e configurações do TransducerChannel TEDS, que podem ser vistos nas informações: Channel Numbers of TIM, ID of Channel No. 1, Name of Channel No.1, ID of Channel No. 2 e Name of Channel No.2.

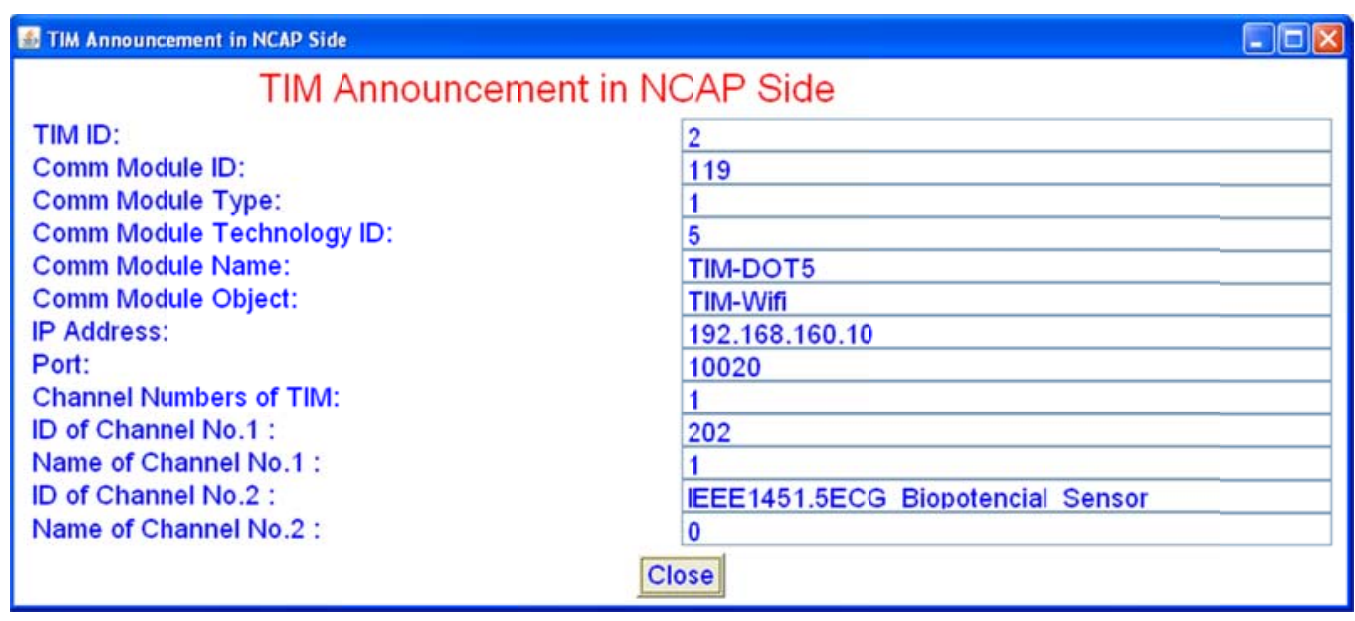

Figura 4.30 - Anúncio do PHY TEDS do WTIM.

O anúncio do WTIM ao NCAP, realizado através de um método PublishSubscriber, oferece alto grau de desacoplamento entre os publicadores e 
consumidores, pois cada nó da rede não teve a necessidade de conhecer a existência dos outros possíveis nós. Outro ponto a ser mencionado foi a verificação do modo de transmissão Only when commanded do WTIM, ou seja, o nó NCAP do NIST respeitou a configuração feita no nó WTIM, pois os dados somente foram encaminhados quando o sistema fez algum tipo de requisição.

A terceira parte correspondeu à descoberta do WTIM pelo NCAP, ou seja, o computador ao receber as informações provenientes do WTIM, registrou esses dados em um banco de dados próprio, como forma de estabelecer mais pedidos de informações e dados.

A quarta etapa para a comunicação estabeleceu o início do pedido de TEDS do NCAP ao WTIM. Nessa operação foram pedidas as informações pertinentes do Meta TEDS, do TransducerChannel TEDS e do User's Transducer Name TEDS. No caso do WTIM foram apresentadas as seguintes informações: Manufacturer ID, Version of TEDS, Number of Channel e Serial Number. Esses dados podem ser vistos na figura 4.31 .

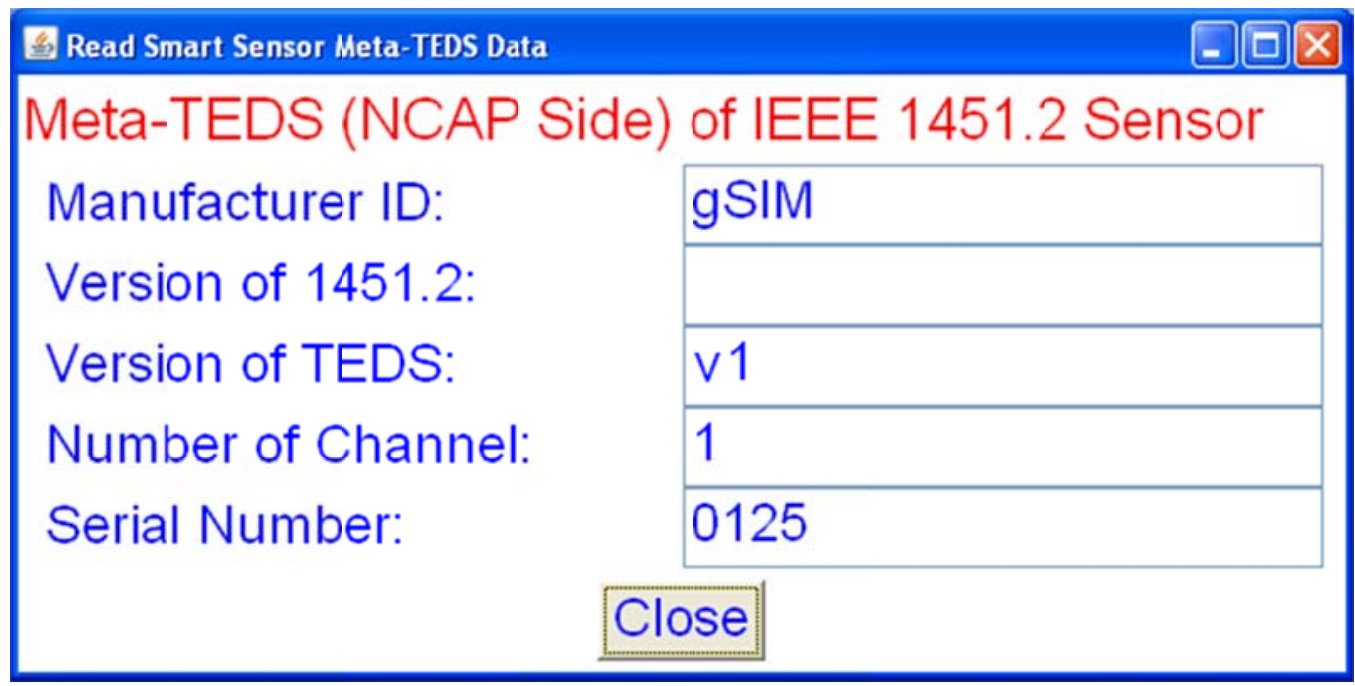

Figura 4.31 - Recebimento da informação do TEDS do WTIM.

A última etapa foi a requisição de dados. Mais uma vez, essa foi realizada através de uma conexão cliente-servidor $T C P / I P$ e o $N C A P$ requisitouos dados 
provenientes do cada sensor de frequência cardíaca. Como já mencionado o WTIM utilizou o modo de amostragem Immediate operation, ou seja, somente quando requisitados os dados foram amostrados e transmitidos ao NCAP. A figura 4.32 apresenta a leitura do medidor de frequência cardíaca igual a 71 batimentos por minuto.

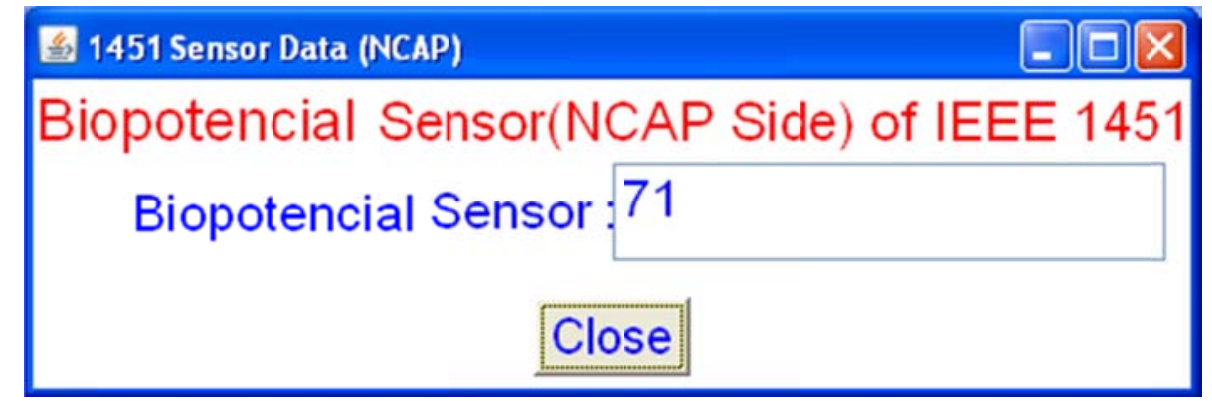

Figura 4.32 - Transmissão dos dados coletados pelo medidor de frequência cardíaca.

Cabe ressaltar que nem toda informação dos TEDS configurado no STIM e WTIM são apresentadas. Partes desses dados são de configurações internas, de ajuste nos modos de transmissão, de limites de medição, de tempos, entre outros.

Outro ponto a ser salientado é que o framework de Song e Lee (2006) auxiliou tanto na verificação como no desenvolvimento do $N C A P$ desse projeto, mostrado nos subcapítulos 4.4.1. Em específico, a programação do $N C A P$ utilizou o modelo de comportamento dinâmico que deve ser realizado entre os TIMs e o NCAP. Além disso, o software $N C A P$ foi inspirado na interface de apresentação dos dados coletados, sejam de TEDS, ou de valores amostrados.

\subsection{Integração da rede de sensores para engenharia biomédica}

A figura 4.33 apresenta a integração dos dois módulos sensores, STIM e $W T I M$, com o NCAP. A começar pelo STIM é possível verificar, na figura, os 
acelerômetros, para a medida de ADM, conectados a placa do microcontrolador AT90USB162. Esse sistema microcontrolado se conectou diretamente ao NCAP, um netbook, através da interface fiada USB. Dentre as vantagens da conexão através desse barramento está a alimentação de tensão e corrente propiciada pela porta USB 2.0. Segundo esse padrão é possível uma conector $U S B$ de dispositivo, fornecer uma tensão de $5 \mathrm{~V}$ com corrente de até $0,5 \mathrm{~A}$. Esse valor foi suficiente para alimentar a placa do processador e os acelerômetros. Ao lado do STIM, verifica-se um eletrogoniômetro utilizado para caracterizar e calibrar os acelerômetros.

Junto ao STIM também se encontra o sistema WTIM. Nesse nó sensor são encontrados um par de eletrodos Kendall-LPT. Esses são devidamente colocados no paciente, nos braços direito e esquerdo, contando com a alocação da pulseira de referência na perna esquerda. Os sinais do paciente foram amplificados pelo circuito condicionador de biopotenciais, apresentando pela caixa metálica. Esse circuito foi alimentado com um bateria de $9 \mathrm{~V}$, o que atenuou interferências oriundas da rede. Uma vez condicionado o sinal, ele foi amostrado no microcontrolador ATmega8535. Esse processador foi responsável pelo envio das informações padronizadas através do protocolo Wifi. Uma vez que esse sistema não estava conectado através de fios com o NCAP, fez-se necessário alimentá-lo através de uma fonte externa, como apresentado na figura 4.33 .

Para a transmissão Wifi, como dito anteriormente, utilizou-se o módulo Wifly GSX 802.11 b/g conectado do WTIM. Esse módulo tem como característica verificada o baixo consumo de potência. Em atividade esse módulo consomiu em média $100 \mathrm{~mW}$, enquanto que no modo de dormência somente $10 \mu \mathrm{W}$. Para verificar o alcance da transmissão sem fio foram realizados alguns testes preliminares sem grande rigor. Com sua potência máxima de transmissão, $18 \mathrm{dBm}$ (aproximadamente $63,1 \mathrm{~mW}$ ), foi possível transmitir sem perda de informação a uma distância de 50 metros em visada direta. Já em ambientes fechados, essa distância não chegou a 20 metros. 


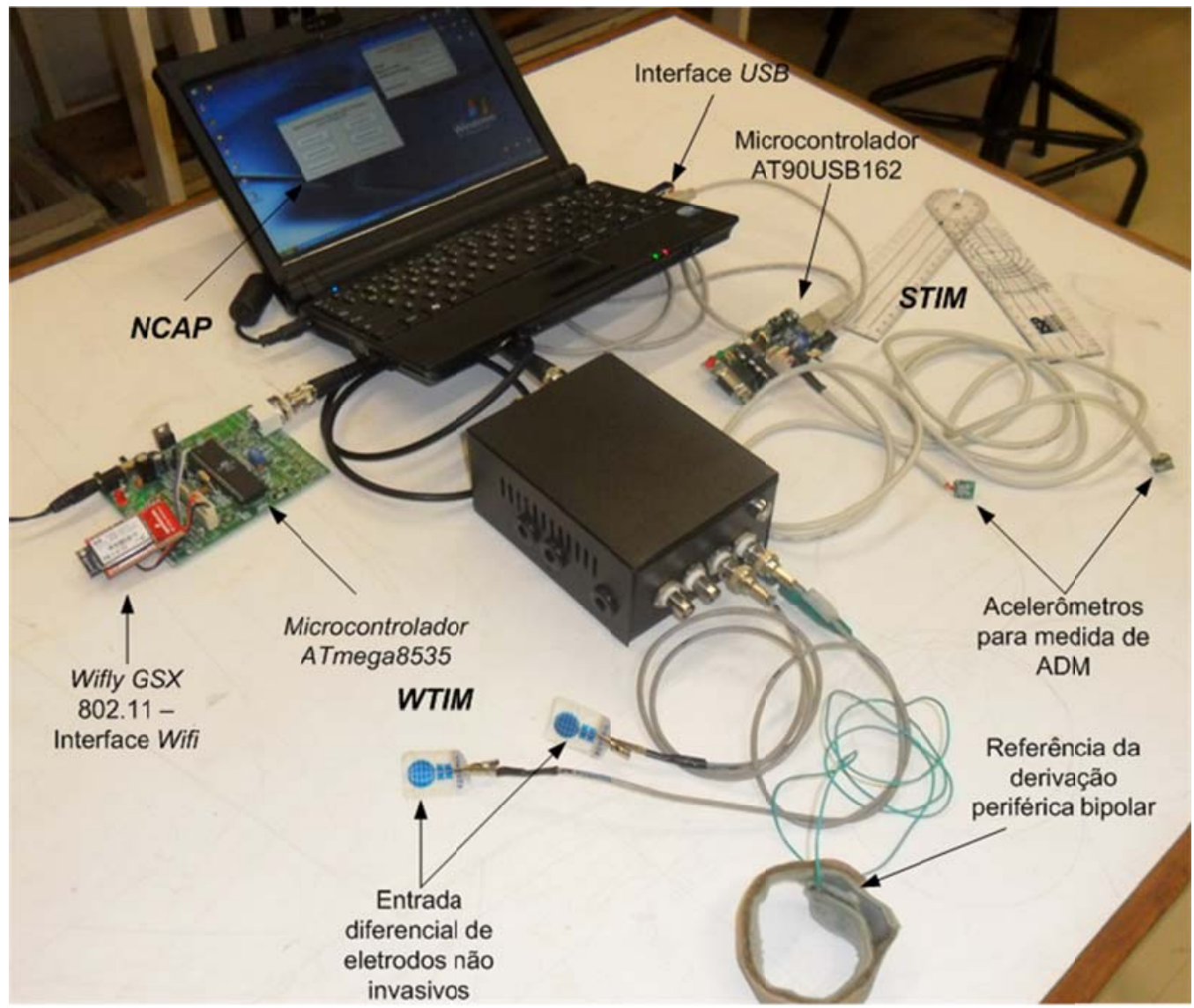

Figura 4.33 - Rede de sensores com STIM, WTIM e NCAP.

Para o teste de integração foi utilizado um netbook (Intel Atom com clock de $1,6 \mathrm{GHz}$ e $2 \mathrm{~GB}$ de memória $R A M$ ) como plataforma de hardware do NCAP. Nesse computador estava associado o software NCAP desenvolvido em linguagem Java. Foi utilizada essa plataforma, pois nela há um dispositivo Wifi embutido, bem como portas USB2.0. Tal fato remete a questão da interoperabilidade pretendida no IEEE1451. Visto que essas interfaces de comunicação são comumente encontradas em computadores, notebooks, celulares, tablets e PDAs (Personal digital assistants). Além disso, é comum que haja conexão desses equipamentos com redes externas, a exemplificar pela Internet. De modo a permitir a comunicação dos nós sensores com uma rede ubíqua.

Com o teste de integração foi verificado o funcionamento das partes que compõem a rede de sensores, tal como caracterizado anteriormente de forma 
separada. Através dessa integração foi obtida uma rede de sensores para engenharia biomédica. Nesse sistema foram constatadas as principais características que definem uma rede de sensores (ILYA; MAHGOUB, 2004). Dentre essas estão: endereçamento, que é a capacidade de cada sensor ter um endereço único; agregação dos dados, que corresponde à possibilidade do módulo sumarizar os dados coletados pelos sensores; capacidade de responder a consultas; auto-organização da rede, visto na capacidade da rede em continuar a executar sua função mesmo sem um de seus sensores; e facilidade de instalação. 


\section{DISCUSSÕES}

A partir da arquitetura projetada e dos resultados obtidos foi possível realizar uma avaliação das partes que compõem a rede de sensores. Constituem essa: os dois sensores biomédicos propostos, os sistemas microcontrolados e suas respectivas interfaces de comunicação USB e Wifi; a normatização dos dados dos sensores por meio do protocolo IEEE1451; e o acesso local das informações através do software NCAP e também através de Web Services, remotamente.

\subsection{SENSORES BIOMÉDICOS E SISTEMAS MICROCONTROLADOS}

A proposta de aplicar acelerômetros para auxiliar as medidas de eletrogoniometria não é inédita. Alguns trabalhos também possuem abordagem semelhante, seja utilizando esses sensores para medidas de ADM (SOARES et al., 2010), ou para analisar o comportamento do movimento humano (SILVA, 2007). Nesse sentido esse projeto se propôs a caracterizar de forma mais rigorosa a utilização desses sensores na medida de ADM. Por isso o maior detalhamento dos passos para análise estatística dos dados no capítulo 4 . Tal análise demonstrou que a incerteza da medida desses acelerômetros está dentro da incerteza dos equipamentos utilizados em eletrogoniometria, não ultrapassando $1^{\circ}$. Dessa forma fica evidente a viabilidade da utilização de acelerômetros em medidas de ADM. Além disso, cabe ressaltar que esses sensores são componentes de baixo custo. No caso do sensor MXD2125GL, parte principal do módulo MX2125, seu valor está na casa da unidade de reais.

Alguns pontos podem ser discutidos em relação ao uso desses acelerômetros. Primeiramente há uma dificuldade de alocação desses sensores no braço. Em grande medida devido ao MX2125 possuir somente dois eixos. Através de um sensor de três eixos seria possível obter o valor de ADM com menor influência do posicionamento dos acelerômetros, entretanto com aumento do custo computacional. Possíveis 
sensores que atenderiam tal especificação são: o AH-6100LR da Epson Toyocom e o MMA7260QT da Freescale.

Cabe ressaltar que os resultados obtidos com a utilização dos acelerômetros podem ser utilizados em aplicações de Tecnologia Assistiva. Pois é do conceito da Tecnologia Assistiva englobar todos os recursos e serviços que ajudam a ampliar, manter ou melhorar as habilidades funcionais de pessoas com deficiência, proporcionando a elas inclusão e determinado grau de independência (BERSCH; TONOLLI, 2011). Ou seja, através da inclusão de sensores acelerômetros seria possível construir equipamentos, que ajudassem na melhoria da qualidade de vida e na inclusão de pessoas com limitações físicas e/ou idosos.

Em relação ao circuito de condicionamento de biopotenciais cabe verificar que a aplicabilidade do sistema não se restringe somente a medidas de ECG. Pois o circuito elétrico projetado e prototipado permite amplificar sinais de unidades de microvolts até dezenas de milivolts. Além disso, possui uma banda que permite a amplificação de sinais de ECG, $E \mathrm{G}^{18}$, e de uma parcela significativa de EMG. A vantagem desse tipo de abordagem é a possibilidade de ter um hardware único, que possibilite ajustar ganhos de acordo com o tipo de pesquisa e desenvolvimento que se queira. Por outro lado, essa mesma característica traz desvantagens caso se queira um ajuste fino. Para tanto um hardware dedicado permitiria ajustes mais sensíveis nas faixas de tensão medidas para cada sinal biológico. Um hardware dedicado também possibilitaria compactar o circuito, bem como aumentar o número de canais por placa.

Dos testes realizados com diferentes tipos de eletrodos não invasivos, percebeu-se que os eletrodos do fabricante Kendall-LPT possuíram a melhor resposta. Em específico, foi utilizado o eletrodo CARE ${ }^{\mathrm{TM}} 610$ Diagnostic Tab Electrodes, esse elemento faz parte da linha de eletrodos de diagnóstico do fabricante.

Para a aquisição dos sinais foram utilizados os microcontroladores ATmega8535 e AT90USB162. Esses microprocessadores são circuitos de baixo

\footnotetext{
${ }^{18}$ Circuitos de condicionamento de EEG podem ser verificados no site: http://openeeg.sourceforge.net/.
} 
custo, cerca de $\mathrm{R} \$ 12,48^{19}$, que permitem além do condicionamento digital das informações coletadas, a introdução dos TEDS e a transmissão através das interfaces Wifi e USB, respectivamente. Nesse ponto, justifica-se a introdução de um microprocessador a um sensor. Em outras palavras, o custo para transformar um transdutor em um transdutor inteligente é pago através dos benefícios da norma IEEE1451 e também pela facilidade e flexibilidade de operação.

Como já dito, os sistemas microcontrolados também possibilitam a transmissão por diferentes interfaces ou barramentos. No caso do AT90USB162 já havia uma interface USB embutida nos periféricos do microcontrolador. Já para a interface Wifi foi conectado ao microcontrolador ATmega8535 o módulo Wifly GSX $802.11 \mathrm{~b} / \mathrm{g}$. Esse módulo engloba um microprocessador, um amplificador de baixo ruído LNA (Low Noise Amplifier) na recepção, um amplificador de potência $P A$ (Power Amplifier) na transmissão e uma antena integrada tem custo de aproximadamente $\mathrm{U} \$ 50,00^{20}$. Apesar de encarecer o sistema, a transmissão sem fio dá maior mobilidade e flexibilidade ao instrumento.

\subsection{PROTOCOLO IEEE1451}

O STIM e o WTIM foram implementados a partir das bibliotecas e modelos desenvolvidos pelo NIST, especificamente as desenvolvidas por Song e Lee (2006) (2008). A possibilidade de reutilização de frameworks produziu maior agilidade na incorporação de sensores comuns à norma IEEE1451. O objetivo da divulgação desses frameworks é reduzir as barreiras para a adoção da norma e estimular pesquisas acerca do protocolo. Muito embora sempre haja necessidade de se restringir a utilização do código e adaptá-lo as características do hardware desenvolvido.

Da leitura atenta das normas do IEEE1451, bem como do desenvolvimento das redes de sensores, foi comprovado que não há restrição de componentes ou de circuitos para os transdutores inteligentes. Isso permitiu que fosse construída uma

\footnotetext{
${ }^{19}$ Valor fornecido pelo distribuidor Farnell Newark em 10 de novembro de 2011.

${ }^{20}$ Valor fornecido pelo distribuidor Sparkfun em 10 de novembro de 2011.
} 
rede utilizando sensores não "convencionais", como é o caso do eletrogoniômetro e do medidor de frequência cardíaca. Tal fato reflete uma questão fundamental do IEEE1451, no qual o fabricante de transdutores deve se concentrar exclusivamente no desenvolvimento do seu produto.

Outra propriedade do IEEE1451, citada no capítulo 2, é a interoperabilidade. $\mathrm{Na}$ rede de sensores biomédicos, o STIM e WTIM se comunicam através de barramentos independentes de fabricante como é o caso do USB e do Wifi. Tais meios estão presentes em todos os tipos de computadores pessoais e notebooks atualmente. Fato também característico do IEEE1451 é o particionamento do sistema em blocos. Para exemplificar tal fato, basta retomar o próprio método de desenvolvimento da rede de sensores, no qual foram detalhados e projetados isoladamente os sensores STIM, WTIM e o processador de rede NCAP.

A perspectiva do IEEE1451 é possibilitar ao usuário final comprar transdutores e simplesmente através de uma conexão USB, Ethernet, Wifi, entre outras, ler ou controlar os valores dos sensores ou dos atuadores. Em outras palavras, a finalidade do IEEE1451 é atender a um mercado que se fragmentou devido às inúmeras especificações ou normas geradas pelos fabricantes de transdutores ou barramentos. Tal fato impediu e ainda impede que se ampliem as aplicações de sensores em ambientes residenciais, industriais, agrícolas ou médicos.

\subsection{NCAP E WEB SERVICES}

A programação em Java do $N C A P$, no ambiente Eclipse, permitiu que se criasse uma interface com o usuário de forma a possibilitar o acesso local das informações oriundas dos sensores. Embora o NCAP envie os comandos para os sensores e apresente as informações recebidas, o software desenvolvido não é capaz de atender todo o tipo de interface do IEEE1451. Em outras palavras, o escopo desse projeto foi o de criar um sistema $N C A P$ padronizado, mas não genérico.

Para o acesso remoto foi utilizado o framework Axis2 para Web Services. Essa implementação do protocolo $S O A P$ encapsula toda a teoria acerca de Web Services, 
inclusive as tecnologias $X M L, S O A P, W S D L$ e $U D D I$. A integração de um $W e b$ Service com o IEEE1451 favoreceu a interoperabilidade do sistema.

Outra questão prática refere-se ao protocolo sobre o qual as mensagens SOAP trafegam. A arquitetura projetada pelo $W 3 C$ especifica que solicitações e respostas $X M L$ trafeguem por meio dos protocolos: HTTP, FTP, SMTP, TCP puro, entre outros. Na prática o $X M L$ trafega prioritariamente sobre o HTTP, pois esse protocolo é dominante na $W e b$ e é suportado por diferentes plataformas sem que ocorram restrições ao seu conteúdo. 


\section{CONCLUSÕES E TRABALHOS FUTUROS}

\subsection{CONCLUSÕES}

No presente trabalho de dissertação foi apresentado o desenvolvimento de uma rede de sensores biomédicos baseado no padrão IEEE1451. Para tanto foram utilizadas ferramentas abertas e padronizadas. Como forma de demonstrar a abrangência do IEEE1451 foram desenvolvidos dois sensores distintos que se conectavam através de um meio com fio e um meio sem fio. Esses nós sensores também possuem como qualidade o acesso remoto através de um Web Service.

Compondo a rede de sensores foi projetado e implementado um nó STIM inspirado no trabalho de Song e Lee (2008b). O hardware desse nó foi desenvolvido utilizando um microcontrolador AT90USB162, de baixo custo, e seu software foi programado através das linguagens ANSI $\mathrm{C}$ e $\mathrm{C}++$. Distinguindo-se do trabalho de Song e Lee (2008b), foi acrescida a comunicação USB em detrimento da serial $R S$ 232. Tal fato agregou uma característica inovadora à dissertação, pois os trabalhos que incorporam o IEEE1451 ao barramento USB (DEPARI et al., 2004) (KUMAR et al., 2011) não utilizaram o padrão IEEE1451.0 como base de configuração para o padrão de interfaceamento IEEE1451.2.

Com relação ao sensor do STIM, foram caracterizados dois acelerômetros Parallax MX2125 para medidas de eletrogoniometria. Esses foram utilizados para a medida de $\mathrm{ADM}$ da flexão e extensão do cotovelo. Os dados coletados desses sensores foram comparados com as leituras realizadas por um goniômetro. Dessa forma, calculou-se um erro relativo médio de $4 \%$ com incerteza da medida de $0,7^{\circ}$. Esses valores ficaram dentro da incerteza vista na literatura, o que demonstrou a confiabilidade desse sistema para medidas de eletrogoniometria e de fleximetria das articulações.

O outro nó sensor desenvolvido foi o WTIM com base no protocolo de interfaceamento IEEE1451.5. O hardware desse nó constou de um microcontrolador ATmega8535 e de um módulo de transmissão Wifi Wifly GSX 802.11b/g. Esse nó também teve como base os trabalhos de Song e Lee (2006) (2007). As informações 
coletadas por esse nó foram transmitidas por rádio frequência, utilizando o protocolo Wifi $(802.11 \mathrm{~b} / \mathrm{g})$. Para a transmissão dos dados foram abertas conexões TCP/IP, que permitiram a comunicação conforme o modelo cliente-servidor. Através desse enlace foi possível que o $N C A P$ requisitasse os valores dos TEDS e dos dados coletados.

Acerca do sensor do WTIM, foi projetado e prototipado um circuito de condicionamento de biopotenciais, que permitiu amplificar, filtrar e condicionar sinais coletados por eletrodos não invasivos. Dentre os elementos que constituem esse circuito se encontram: um amplificador diferencial, um filtro passa-altas, um amplificador não inversor, um filtro rejeita-faixas $(60 \mathrm{~Hz})$ e um filtro passa-baixas. No sistema em questão esse circuito foi responsável por amplificar sinais de ECG, coletados através da derivação periférica bipolar D1. Esse sinal foi amostrado pelo microcontrolador, que por sua vez forneceu o valor da frequência cardíaca através da contagem de tempo entre duas ondas $\mathrm{R}$.

A validação do WTIM foi realizada através do framework desenvolvido por Song e Lee (2006) (2007). Esse software aberto ${ }^{21}$ foi utilizado para caracterizar concomitantemente o NCAP e o WTIM da rede de sensores biomédicos, como visto no capítulo 4.6. Por meio dessa validação foi possível garantir o funcionamento do IEEE1451.0 e do IEEE1451.5, bem como da qualidade dos testes e resultados obtidos.

Para completar o sistema IEEE1451 foi desenvolvido um NCAP, escrito em linguagem Java ( $S D K$ 6.0) no ambiente integrado de desenvolvimento Eclipse, rodando em um sistema operacional Windows Xp. Esse software foi responsável pela transmissão dos comandos para envio das informações dos TEDS e dos dados amostrados pelos sensores. Além disso, foi responsabilidade do $N C A P$ decodificar os $T E D S$ e apresentar localmente as informações dos sensores e dos dados coletados.

Por último foi desenvolvido em cima do NCAP um sistema para acesso remoto através de um Web Service. Essa tecnologia permitiu que as informações apresentadas localmente pelo $N C A P$ fossem disponibilizadas na rede de forma a viabilizar a monitoração remota da informação dos sensores e dos dados coletados.

${ }^{21}$ An Open Implementation of IEEE 1451 - http://sourceforge.net/projects/open1451/files/. Acesso em: 20/11/2011. 
Vale ressaltar que a tecnologia Web Services permite que praticamente todo tipo de plataforma de software e hardware tenha acesso às informações do $N C A P$, sem que ocorram problemas e restrições de firewalls.

Acerca do funcionamento do IEEE1451, verificou-se que, de fato, há uma distinção entre o projeto do sensor/atuador e da rede de controle. Isso ocorre devido à separação do módulo TIM e do módulo NCAP. No caso da rede de sensores desenvolvida, o STIM e o WTIM se comunicavam de maneiras distintas com o NCAP. Esse, por sua vez, fez uma interface com a rede de controle Internet de maneira totalmente independente da escolha dos sensores.

Esse trabalho também mostrou que o padrão IEEE1451 é capaz de ser integrado a diferentes plataformas de hardware, sistemas operacionais e linguagens de programação. Dessa forma foi realçada a possibilidade de interoperabilidade entre distintos transdutores.

Embora ainda haja restrição para o uso do IEEE1451, em grande medida por ser um protocolo que ainda está em processo de modificações e acomodações, verificou-se que há potencial em sua utilização. Esse reside na introdução de informações relativas aos transdutores no TEDS e na forma plug and play de conexão. Acredita-se que a tendência dos transdutores é a de se tornar sistemas pervasivos, seja no ambiente industrial, da agricultura, residencial, ou médico. Nesse sentido foi meta desse trabalho tentar se antecipar às tendências da microeletrônica.

O fato de algumas normas da família IEEE1451 ainda não estarem totalmente definidas e estabelecidas, justifica o desenvolvimento e as pesquisas no meio acadêmico. Nesse sentido, adquirir conhecimento sobre o protocolo e propor possíveis modificações ou melhorias foram propostas desse trabalho.

A aplicação de um protocolo de rede de sensores, por si só, já é um grande avanço em qualquer sistema de automação. Em especial, o desenvolvimento desse protocolo em aplicações não canônicas, ou seja, não triviais, demonstra a possibilidade de implementação em outros tipos de sistemas. A exemplificar por aplicações em: automação e controle de processos, monitoração ambiental, computação pervasiva, etc. 
Os resultados obtidos incluem o desenvolvimento de hardwares dedicados ao avanço de redes de sensores em Engenharia Biomédica. Vale novamente ressaltar uma característica fundamental dos sensores IEEE1451, a capacidade de se conectar de maneira plug and play. Deste modo, o utilizador apenas tem que fazer a conexão e o sistema automaticamente detecta, configura e comunica sem a necessidade de interação humana. Essa nova abordagem para rede de sensores apresenta vantagens associadas à flexibilidade de configuração e principalmente ao custo em relação ao limitado número de sistemas similares que já existem no mercado.

Por fim cabe, resumidamente, mencionar as contribuições deste trabalho que foram: a pesquisa e revisão bibliográfica das normas do IEEE1451; o desenvolvimento de uma rede de sensores utilizando as normas IEEE1451.0, IEEE1451.2 e IEEE1451.5; a proposta de atualizar o campo de pesquisa sobre o protocolo IEEE1451, com a incorporação da norma IEEE1451.0; o projeto de um circuito condicionador de biopotenciais de baixo custo; a caracterização e análise de acelerômetros como sensores de ADM; o desenvolvimento de um hardware embarcado para sensores biomédicos; e, por último, a programação de um NCAP capaz de realizar a comunicação entre os dispositivos USB e Wifi, local e remotamente.

\subsection{TRABALHOS FUTUROS}

As possibilidades de desenvolvimento a partir do IEEE1451 são inúmeras. Entretanto alguns pontos podem ser explorados para que se impulsione a utilização dessa norma. O principal ponto é aumentar a utilização do TEDS, com o desenvolvimento de aplicações que utilizem TEDS de calibração, TEDS de localização geográficos, entre outros. Em especial, a utilização da localização geográfica através de um sistema de gerenciamento de posição (GPS) permitiria que sensores móveis tivessem sua localização transmitida através de um sistema já normatizado.

Como foi apontado no capítulo 5, o desenvolvimento de TEDS não é trivial. Uma possibilidade para a otimização dessa etapa crucial seria a de desenvolver 
softwares que criassem e editassem TEDS, conforme o trabalho de Manda e Gurkan (2009).

Em relação às camadas físicas de transmissão, o IEEE1451 deixa espaço para que haja a incorporação de outros meios de comunicação. Dentre eles destacam-se o sistema General Packet Radio Service (GPRS), que utiliza a rede de telefonia celular para a transmissão de dados (NASCIMENTO et al., 2011).

Com relação ao hardware para os sensores vale destacar a tendência para a utilização das plataformas Motes, que possuem baixo consumo de energia, baixo custo, são pequenos e incorporam sistemas operacionais que favorecem o desenvolvimento de serviços em tempo real e métodos de redução de consumo através de ciclos de sleep mode (latência do sistema). Alguns exemplos desses hardwares são: Crossbow IRIS, Crossbow MICAz, Crossbow MICA2, Crossbow Imote2, Crossbow TelosB, Atmel AVR Raven, Scatternode, Shimmer e Sun SPOT.

Através da incorporação desses sensores com os conceitos do IEEE1451, especificamente o IEEE1451.5, poder-se-ia verificar a viabilidade desse protocolo em sistemas que ainda não possuem normatização quanto à comunicação. Além disso, cabe verificar possíveis alternativas para que o IEEE1451 seja incorporado a esses Motes de forma a manter o baixo consumo de energia e não sobrecarregar o processador ou o transmissor (TORRI, 2008).

Dentro do protocolo IEEE1451.5 também são possibilidades de trabalho, agregar os protocolos ZigBee, 6LoWPan e UWB. O primeiro, principal meio de comunicação de rede de sensores sem fio, permitiria verificar o funcionamento do IEEE1451 em diferentes topologias (Mesh, Star, Cluster Tree). Já o 6LoWPan, IPv6 over Low power Wireless Personal Area Networks, permite a utilização de protocolos IP para comunicação de alto nível entre o controlador e os diversos sensores da rede. A escolha do $U W B$ reside no fato que nessa tecnologia é possível obter altas taxas de transmissão através de transceptores de baixo custo, baixa potência e baixa susceptibilidade a interferência.

Em relação ao sistema desenvolvido, alguns aspectos poderiam ser incorporados ao trabalho. A exemplificar por um banco de dados que armazene todos os dados coletados pelo software de monitoramento $N C A P$. Outro ponto que poderia 
ser modificado é a transmissão dos dados da curva de ECG e não somente a frequência cardíaca do paciente. Também poderiam ser transmitidos os sinais coletados de EMG e EEG, pois o condicionador de sinais desenvolvido permite amplificar o espectro que incorpora esses dois tipos de sinais. Além do mais, como se pretende desenvolver um sistema para engenharia biomédica, outros parâmetros médicos podem ser utilizados como a transmissão da pressão arterial, da temperatura ou da medida de oximetria de pulso do paciente.

Ainda sobre a aquisição de sinais de ECG, também é uma possibilidade de continuidade do trabalho o desenvolvimento de filtros digitais que atenuem os artefatos do sinal, em específico o ruído de $60 \mathrm{~Hz}$. Para isso poderia-se analisar a resposta do sinal de ECG aos filtros FIR (Finite Impulse Response) ou IIR (Infinite Impulse Response) e suas distintas aproximações.

No âmbito das redes de sensores para engenharia biomédica, cabe avaliar os nós sensores ETag2 desenvolvidos pela Universidade da Virginia. Esses nós sensores sem fio são utilizados para verificar sinais vitais de pacientes, como ECG, oximetria de pulso e temperatura. Outro sistema de Medical Healthcare a ser analisado é o CodeBlue. Essa plataforma desenvolvida pela Universidade de Harvard trabalha em cima de um Mote com um sistema operacional TinyOS, medindo valores de oximetria de pulso e de duas derivações de ECG.

Outra possibilidade de trabalho é a integração do IEEE1451 com ISO/IEEE 11073, norma que estabelece interoperabilidade entre equipamentos médicos. O objetivo é permitir a transferência de informações médicas entre equipamentos, de modo a facilitar que essas sejam armazenadas, recuperadas e processadas em tempo real. Dessa norma é possível encontrar bibliotecas como a Antidote e a OpenHealth.

Outra possível integração do IEEE1451 é com o padrão HL7 (Health Level 7) e com a linguagem normatizada para sensores SensorML. Esses padrões têm como objetivo padronizar a linguagem médica e a descrição espacial do sensor, respectivamente. Ambos os padrões permitiriam ampliar a interoperabilidade funcional e semântica dos sensores projetados. 


\section{TRABALHOS APRESENTADOS}

Abaixo segue uma lista de trabalhos apresentados acerca do protocolo IEEE1451 ou publicados durante o desenvolvimento desse mestrado, no qual estão incluídos alguns congressos e o depósito de uma patente.

BECARI, W. SAPP - Sensores e Atuadores Plug and Play. 2005. In: $13^{\circ}$ Siicusp - Seminário Internacional de Iniciação Cientifica da USP, 2005.

CORSO, J. A.; BECARI, W. Plug and Play Network Sensor Architecture. $5^{\circ}$ Congresso Ibersensor, Montevideo, Uruguay, 2005.

BECARI, W. Instrumentação Inteligente em Sistemas Dedicados. 2006. In: $14^{\circ}$ Siicusp - Seminário Internacional de Iniciação Cientifica da USP, 2006.

BECARI, W.; CORSO, J. A.; FERNANDEZ, F. J. F. Sensors as alternative way for teaching Embedded Systems and Microelectronics, ICEE International Conference on Engineering Education, Portugal, Coimbra, 2007.

BECARI, W.; RAMIREZ, J.; HIDALGO; D. F.; FALLA; P. H. Desenvolvimento de sensores e sistemas eletrônicos com interface Web para detecção de gases poluentes e adulteração de combustíveis. XVIII Congresso Brasileiro de Automática. Bonito - MS, 2010.

BECARI, W.; RAMIREZ, J. Rede de Sensores utilizando o protocolo IEEE1451. Escola de Instrumentação em Astronomia e Eletrônica Orgânica. São Carlos, 2010.

OLIVEIRA, G.; DOTTO, M.; HENRIQUE, P.; MAIA, T; BECARI, W. Sistema de sensoriamento remoto de umidade e temperatura do solo para irrigação de precisão. $18^{\circ}$ SIICUSP, São Paulo, 2010.

OLIVEIRA, G.; DOTTO, M.; HENRIQUE, P.; MAIA, T; BECARI, W. Sistema de sensoriamento remoto de umidade e temperatura do solo para irrigação de precisão. $12^{\circ}$ SICT, FATEC-SP, São Paulo, 2010. 
RAMIREZ, J. ; BECARI, W.; FILHO, J. F. J. Sistema de controle automático antiderrapante aplicado a um calçado. Depósito de patente, 2011.

NASCIMENTO, B. J.; PONTES, F. O. A.; ALNCANTARA, M. O.; BECARI, W. Sistema de Monitoração Remota de Eletrocardiograma Através da Rede de Telefonia Celular. 13 SICT, FATEC-SP, São Paulo, 2011.

NASCIMENTO, B. J.; PONTES, F. O. A.; ALNCANTARA, M. O.; BECARI, W. Sistema de Monitoração Remota de Eletrocardiograma Através da Rede de Telefonia Celular. $19^{\circ}$ SIICUSP, São Paulo, 2011. 


\section{REFERÊNCIAS}

ALBINADER NETO, J. A. Web Services em Java. Rio de Janeiro: Brasport, 2006.

ALONSO, G. et al. Web Services: Concepts, Architectures and Applications. Springer, 2004.

ARRUDA JR., C. R. E. Context Kernel: um Web Service baseado nas dimensões de informação de contexto. 2003. 85p. Dissertação (Mestrado) - Institutp de Ciência Matemáticas e de Computação da Universidade de São Paulo. São Paulo, 2003.

AXELSON, J. USB complete: everything you need to develop custom USB peripherals. Lakeview Research, $2^{\mathrm{a}}$ edition, 2001.

BAILEY, J. J.; BERSON A. S.; GARSON A.; et al. Recommendations for standardization and specifications in automated electrocardiography: bandwidth and digital signal processing: A report for health professionals by an ad hoc writing group of the committee on electrocardiography and cardiac electrophysiology of the Council on Clinical Cardiology, American Heart Association. Circulation p.81-730, 1990.

BATISTA, E. A. Emprego da Tecnologia Java para Implementar a Parte Lógica de um Processador de Aplicação com Capacidade de Operar em Rede de Comunicação (NCAP), em Conformidade com o Padrão IEEE 1451. Dissertação (Mestrado), FEIS-UNESP. Ilha Solteira: 2004.

BECARI, W.; CORSO, J. A.; FERNANDEZ, F. J. F. Sensors as alternative way for teaching Embedded Systems and Microelectronics, ICEE International Conference on Engineering Education, Portugal, Coimbra, 2007.

BERSCH, R.; TONOLli, J. C. Tecnologia Assistiva. Disponível em: $<$ http://www.assistiva.com.br/>. Acesso em: 10 de setembro 2011. 
BRONZINO, J. D. The Biomedical Engineering Handbook. CRC Press, 2000.

CESCHINI, G. W. Proposta de extensões a métodos e ferramentas de desenvolvimento de sistemas de automação distribuídos baseados em modelos dados pela UML e pela IEC 61499. Dissertação (Mestrado) - Escola Politécnica, Universidade de São Paulo, São Paulo, 2008.

CHINNICI, R.; MOREAU, J. J.; RYMAN, A.; WEERAWARANA, S. Web Services Description Language Part 1 Version 2.0 Specification. W3C, Disponível em: <http://www.w3.org/TR/2005/WD-wsd120-20050510>, Acesso em agosto de 2010.

CHONG, Chee-Yee; KUMAR, S. P. Sensor networks: Evolution, opportunities, and challenges. Proceedings of the IEEE, 91(8): p.1247-1256, Agosto de 2003.

CORSO, J. A.; BECARI, W. Plug and Play Network Sensor Architecture. $5^{\circ}$ Congresso Ibersensor, Montevideo, Uruguay, 2005.

DEPARI, A.; FERRARI, P.; FLAMMINI, A.; MARIOLI, D.; SISINNI, E.; TARONI, A. IEEE1451 smart sensors supporting USB connectivity. Sensors for Industry Conference. Proceedings the ISA/IEEE , p. 177- 182, 2004.

DURWARD, R. B., BAER, W. D., ROWE, P. J. Movimento funcional humano: mensuração e análise. São Paulo: Manole, 2001.

ELMENREICH, W.; PITZEK, S. Smart transducers, principles, communication and configuration. IEEE Internacional Conference on Intelligent Engineering Systems, 7., Luxor. Proceedings. p. 510-515, 2003.

ELVERU R. A.; ROTHSteIN J. M.; MilleR P. J.; ROETTGER R. F. Goniometric reliability in a clinical setting. Elbow and knee measurements. Phys Ther, 1988.

FILHO, T. A. S.; SIlvA, A. C. R.; NOGUEIRA, A. L. A. N.; ROSSI, S. R. BATISTA, E. A. Descrição dos TEDS para o controle de motores de asso em 
conformidade com o padrão IEEE 1451. XVIII Congresso Brasileiro de Automática, Bonito-MS. 12 a 16 de setembro de 2010.

GILSINN, J. D.; LEE, K. Wireless interfaces for IEEE1451 sensor networks. SIcon'01, Rosemount, IL, USA, p. 45-50, Novembro 2001.

GODOI, A. C. B. Detecção de potenciais evocados P300 para ativação de uma interface cérebro-máquina. 110 p. (Mestrado) - Escola Politécnica, Universidade de São Paulo, São Paulo, 2010.

GOMES, D. A. Web Services SOAP em Java. São Paulo: Novatec Editora, 2009.

GUYTON, A.C.; HALL, J.E. Tratado de Fisiologia Médica. $11^{\text {a }}$ ed. Rio de Janeiro, Elsevier Ed., 2006.

HIGUERA, J.; POLO, J.; GASULLA, M. A Zigbee wireless sensor network compliant with the IEEE1451 standard. Sensors Applications Symposium, 2009.

IEEE. IEEE Standard for a Smart Transducer Interface for Sensors and Actuators - Transducer to Microprocessor Communication Protocols and Transducer Electronic Data Sheet (TEDS) Formats. IEEE Std 1451.2-1997, Setembro 1998.

IEEE Standard for a Smart Transducer Interface for Sensors and Actuators-Network Capable Application Processor (NCAP) Information Model. IEEE Std 1451.1-1999, Sucedido por 1451.0-2007, 2000.

. IEEE Standard for A Smart Transducer Interface for Sensors and Actuators - Mixed-Mode Communication Protocols and Transducer Electronic Data Sheet (TEDS) Formats. IEEE Std 1451.4-2004, p. 1-430, Março de 2004.

- IEEE Standard for a Smart Transducer Interface for Sensors and Actuators-Digital Communication and Transducer Electronic Data Sheet (TEDS) Formats for Distributed Multidrop Systems. IEEE Std 1451.3-2003, p. 1$175,2004$. 
. IEEE Standard for a Smart Transducer Interface for Sensors and Actuators - Common Functions, Communication Protocols, and Transducer Electronic Data Sheet (TEDS) Formats. IEEE Std 1451.0-2007, p. 1-335, Revisão do 1451.1-1999.Setembro 2007.

. IEEE Standard for a Smart Transducer Interface for Sensors and Actuators Wireless Communication Protocols and Transducer Electronic Data Sheet (TEDS) Formats. IEEE Std 1451.5-2007, p.236, Outubro de 2007.

. IEEE Standard for a Smart Transducer Interface for Sensors and Actuators - Transducers to Radio Frequency Identification (RFID) Systems Communication Protocols and Transducer Electronic Data Sheet Formats. IEEE1451.7, 2009.

- IEEE P1451.0 Website (a). Disponível em $<$ http://grouper.ieee.org/groups/1451/0>. Acesso em: 2 de julho de 2010.

- IEEE P1451.6 Website (b). Disponível em $<$ http://grouper.ieee.org/groups/1451/6>. Acesso em: 2 de julho de 2010.

ILYAS, M.; MAHGOUB, I. Handbook of Sensor Networks: Compact Wireless and Wired Sensing Systems. CRC Press, 2004.

IVLINE, A quick guide of ECG. Disponível em $<$ http://www.ivline.info/2010/05/quick-guide-to-ecg.html $>$. Acesso em: Novembro de 2011.

JONES, L. D.; CHIN, A. F. Electronic Instruments and Measurements, Prentice Hall, Inc., 2a. Edição, 1991.

KAPANDJI, A. I. Fisiologia articular. 6. ed. Rio de Janeiro: Guanabara Koogan, 2007.

KREGER, H. Web Services Conceptual Architecture (WSCA 1.0), IBM Software Group, Março de 2001. 
KUMAR, A.; SINGH, I. P.; SUD, S. K. Energy Efficient and Low-Cost Indoor Environment Monitoring System Based on the IEEE 1451 Standard. IEEE Sensors Journal, vol.11, no.10, p.2598-2610, Outubro de 2011.

LEE, K. Sensor networking and interface standardization. In: Instrumentation and Measurement Technology Conference, 18., 2001, Budapest. IEEE, 2001.

. Introduction to IEEE1451 - Family of Standards. Wireless Sensor Standard Workshop/Meeting - Sensors Expo/Conference. NIST - National Institute of Standards and Technology, 7 de Junho de 2004.

LEE, K.; REICHARDT, M. Open Standards for Homeland Security Sensor Networks. IEEE Instrumentation and Measurement Magazine, Dezembro de 2005.

LEE, K. Smart and Wireless Sensor Standards for Distributed Measurements. Instrumentation \& Measurement Magazine, IEEE Guest Editor, Abril de 2008.

LIPPERT, L. S. Clinical Kinesiology and Anatomy. 4th ed. F.A. Davis Company, 2006.

MANDA, S.; GURKAN, D. IEEE 1451.0 compatible TEDS creation using .NET framework. IEEE Sensors Applications Symposium, p.281-286, 17-19 Fevereiro de 2009.

MEMSIC. MEMSIC Website. Disponível em <http://www.memsic.com/>. Acesso em: 10 de agosto de 2011.

MITRA, E. N.; LAFON, Y. W3C SOAP Part 0 Primer Version 1.2 Specification. W3C. Disponível em: http://www.w3.org/TR/2003/REC-soap12-part0-20030624/, Acesso em: Agosto de 2009.

NASCIMENTO, B. J.; PONTES, F. O. A.; ALNCANTARA, M. O.; BECARI, W. Sistema de Monitoração Remota de Eletrocardiograma Através da Rede de Telefonia Celular. Trabalho de Conclusão de Curso, Fundação Instituto Tecnológico de Osasco, 2011. 
NATIONAL. An Overview of IEEE1451 .4 Transducer Electronic Data Sheet (TEDS). Specifications for all transducers by National Instruments. Disponível em: $<$ http://zone.ni.com/devzone/cda/tut/p/id/3469>. Acesso em: Fevereiro de2011.

NEMETH-JOHANNES, J.; SWEETSER, V.; SWEETSER, D. Implementation of an IEEE-1451.0/1451.5 compliant wireless sensor module. Autotestcon p.364$371,2007$.

NIST. IEEE1451 Protocol Website. National Institute Of Standards and Technology. Disponível em: < http://ieee1451.nist.gov>. Acesso em: Março de 2010.

NORTON, H. Transducer fundamentals. In: Handbook of Transducers. Englewood Cliffs, NJ: Prentice Hall, 1989.

OLIVEIRA, A. L. Instrumentação inteligente baseada em Web Services. Dissertação (Mestrado) - Escola Politécnica, Universidade de São Paulo. São Paulo, 2005 .

ORTOLAN, R. L. Estudo e Avaliação de Técnicas de Processamento do Sinal Mioelétrico para o Controle de Sistemas de Reabilitação. São Carlos, 2002. 113 p. Dissertação (Mestrado) - Depto de Engenharia Elétrica da Escola de Engenharia de São Carlos, Universidade de São Paulo.

PAVEL, R.; TIPEK A. Modern Sensors Handbook. Wiley-ISTE, 2007. 518p.

RAMOS, H. G. A Contribution to the IEEE STD. 1451.2-1997 Revision and Update. Proc IEEE AFRICON Conf. in Africa, Windoek, Namibia, Setembro de 2007.

ROSSI, S. R. Implementação de um Nó IEEE 1451, Baseado em Ferramentas Abertas Padronizadas, para Aplicações em Ambientes de Instrumentação Distribuída. Tese (Doutorado), FE/IS-UNESP. Ilha Solteira, 2005. 
SADOK, E. F.; LISCANO, R. A web-service framework for 1451 sensor networks. Instrumentation and measurement technology conference, Ottawa, Canada, p. 17-19, Maio de 2005.

SAHA, D.; MUKHERJEE, A. Pervasive Computing: A Paradigm for the 21st Century. IEEE Computer, 2003.

SAMAD, T.; MCLAUGHLIN, P.; LU, J. System architecture for process automation: Review and trends. Journal of Process Control, Volume 17, Issue 3, p.191-201, Março de 2007.

SANTOS, J. C. dos. Sistema Eletrônico para aquisição, processamento e armazenamento de sinais. Dissertação (Mestrado) - Escola Politécnica, Universidade de São Paulo, São Paulo, 2005.

SEDRA, A.S.; SMITH, K.C. Microeletrônica. $5^{\text {a }}$ Ed. São Paulo. Pearson Prentice Hall, 2007.

SILVA, T. I. Implementação de um sistema para geração e avaliação de movimentos em pacientes hemiplégicos. Tese de doutorado. UNESP-SP, Ilha Solteira, 2007.

SOARES, F. P.; MENDES, J. P.; SORRENTINO, J. V. M. M.; ALVES, L.; PERES, H. M. P. L. Eletrogoniômetro para aplicações em fisioterapia. Trabalho de Conclusão de curso. Fundação Instituto Tecnológico de Osasco. 2010.

SONG, E.; LEE, K. Object-oriented application framework for IEEE1451.1 standard. Instrumentation and Measurement Technology Conference. Proceedings of the 21st IEEE, Volume 2, 18-20 Maio de 2004.

An implementation of the proposed IEEE1451.0 and 1451.5 standards. Sensors Applications Symposium. Proceedings of the IEEE, p.7277. 2006. 
Wireless Sensor Network Based on IEEE 1451.0 and IEEE

1451.5-802.11. Electronic Measurement and Instruments, 8th International Conference, pp.4-11, Julho de 2007.

. Understanding IEEE1451-networked smart transducer interface standard-what is a smart transducer? Instrumentation \& Measurement Magazine, IEEE, 11, n. 2, p. 11-17, 2008.

. Sensor Network based on IEEE 1451.0 and IEEE p1451.2-

RS-232. Instrumentation and Measurement Technology Conference Proceedings, 2008, p.1728-1733, 12-15 de Maio de 2008.

STWS: A Unified Web Service for IEEE1451 Smart

Transducers. Instrumentation and Measurement, IEEE Transactions on, vol.57, no.8, p. 1749-1756, Agosto de 2008.

SVEDA, M. A design framework for Internet-based embedded distributed systems. 11th IEEE Conference and Workshop on the international Engineerign of computer-based system, p. 113-120, 2004.

TANI, F. K. Proposta de desenvolvimento de transdutores inteligentes baseados na norma IEEE1451 aplicados a redes LonWorks. 151 p. Dissertação (Mestrado) - Escola Politécnica, Universidade de São Paulo, São Paulo, 2006.

TEXAS. Active Filter Design Techniques. In: Op Amps For Everyone: Design Reference. Rev. B. Agosto de 2002.

TORRI, L. B. A norma IEEE 1451 aplicada a redes heterogêneas de sensores sem fio. Trabalho de Conclusão de Curso, Universidade Federal de Santa Catarina, 2008 .

VEGA, C. H. F. Reconhecimento de estados cognitivos em sinas EEG. 101 p. (Mestrado) - Escola Politécnica, Universidade de São Paulo, São Paulo, 2010. 
VIEGAS, V. M. R. Projeto e implementação de um sistema de sensores inteligentes baseado na norma IEEE1451. 2003. 241p. Dissertação (Mestrado) Instituto Superior Técnico da Universidade Técnica de Lisboa, Lisboa, 2003.

VIEGAS, V.; PEREIRA, J. M. D.; GIRÃO, P. S. Using a Commercial Framework to Implement and Enhance the IEEE1451.1 Standard. IMTC 2005 Instrumentation and Measurement Technology Conference, Ottawa, Ontario, CANADA, 17-19 de Maio 2005.

VIEGAS, V.; PEREIRA, J. M. D.; GIRÃO, P. S. .NET Framework and Web Services: A Profit Combination to Implement and Enhance the IEEE1451.1 Standard. IEEE Transactions on Instrumentation and Measurement, vol. 56, no. 6, Dezembro, 2007.

VUOLO, J. H. Fundamentos da Teoria de Erros. São Paulo: Edgar Blücher, 1996.

WEBSTER, J. G. Medical Instrumentation. John Wiley and Sons, 3rd edition, 1998.

WEISER, M. The Computer for the Twenty-First Century. Scientific American, p. 94-10, Setembro 1991.

WOBSCHALL, D. Websensor design-Smart HVAC sensors with an internet address. Proc. Sensors Expo, 2001.

. A wireless gas monitor with IEEE1451 protocol. Proc. SAS,

2006.

. Networked Sensor Monitoring Using the Universal

IEEE 1451 Standard. IEEE Instrumentation \& Measurement Magazine, 2008.

WOBSCHALL, D.; MUPPARAJU, S. Low-power wireless sensor with SNAP and IEEE1451 protocol. IEEE Sensors Applications Symposium, p. 225-227, 2008. 
YONGPING, Xie; YUN, Zhang; ZHIJIA, Yang. A Middleware Framework Based on the IEEE1451 Standards. Wireless Communications, Networking and Mobile Computing, 2007. International Conference p.3119-3122, 21-25 Set. 2007. 


\section{Apêndice A - Simulação do circuito de condicionamento de biopotenciais}

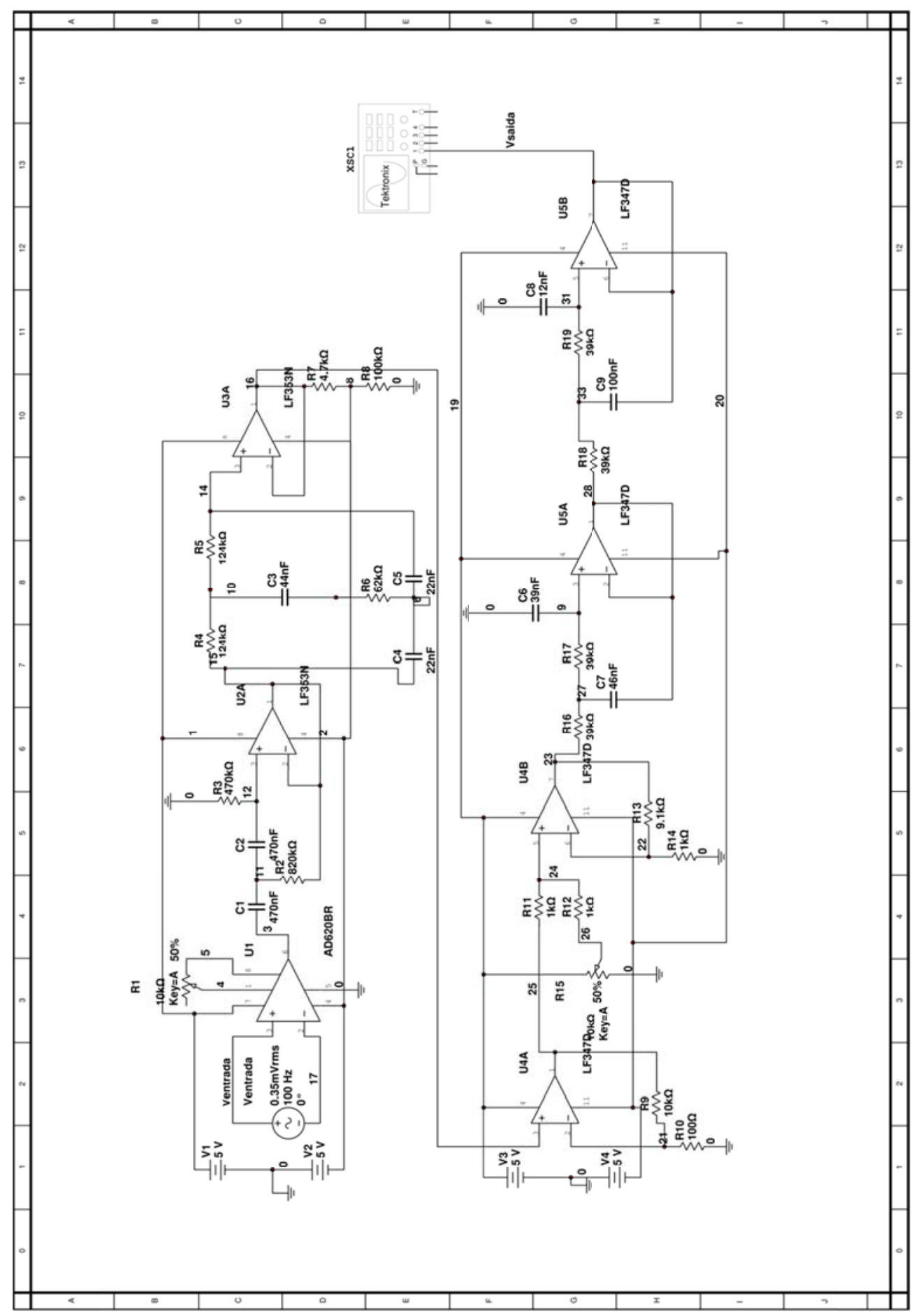




\section{Apêndice B - Simulação do circuito condicionador com Instrumentos Virtuais}

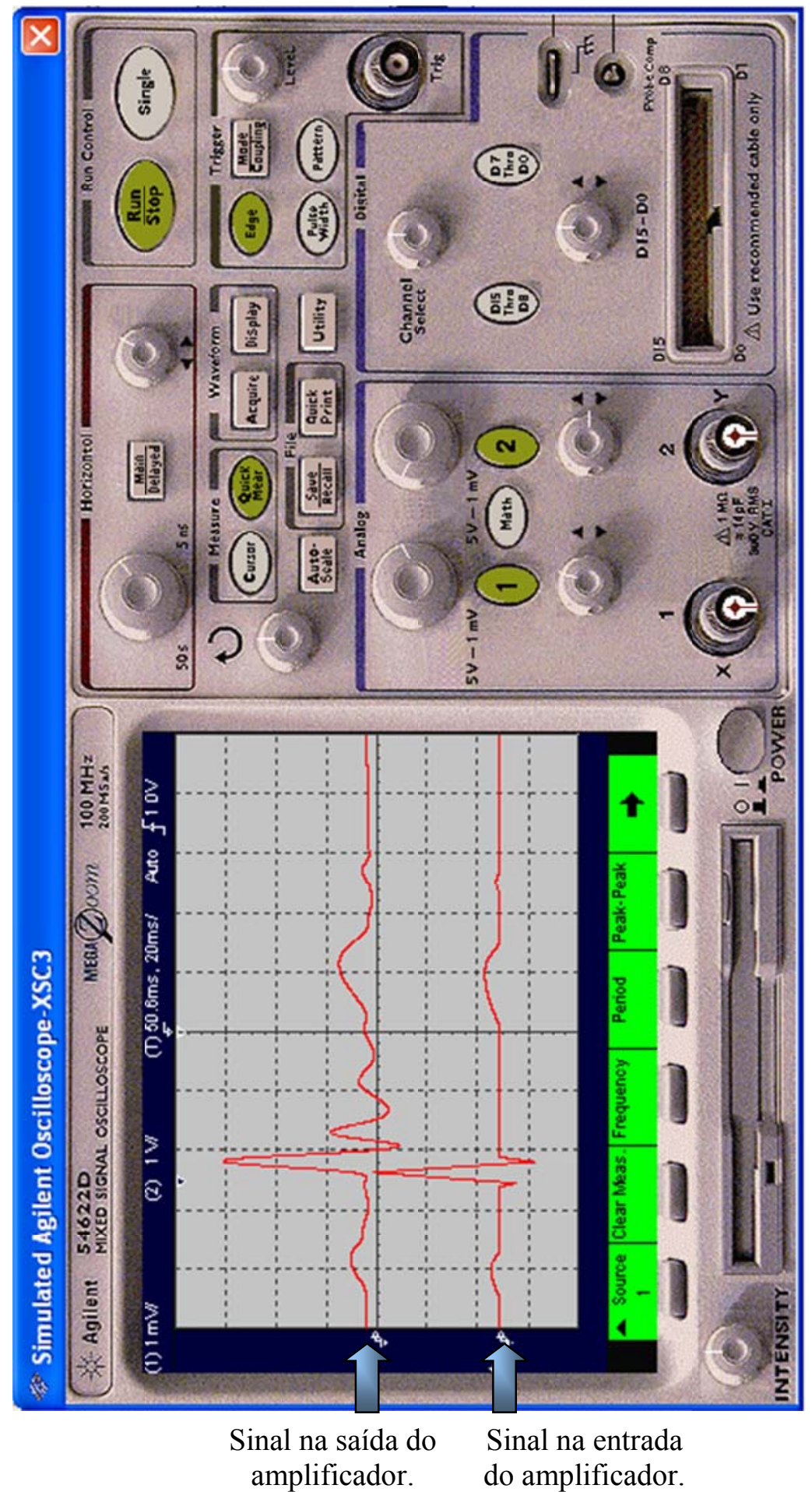




\section{Apêndice C - Esquema do circuito de condicionamento de biopotenciais - Parte 1}
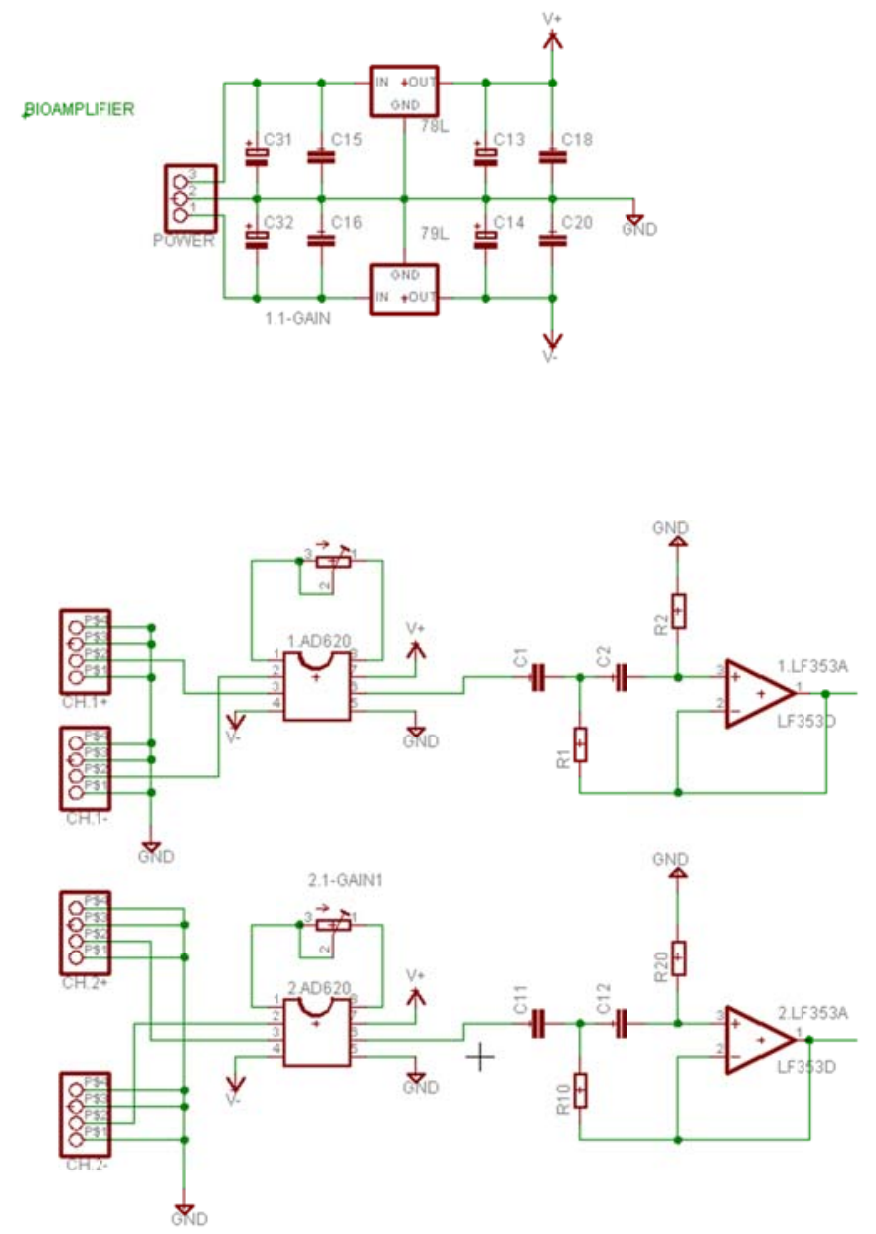


\section{Apêndice D - Esquema do circuito de condicionamento de biopotenciais - Parte 2}

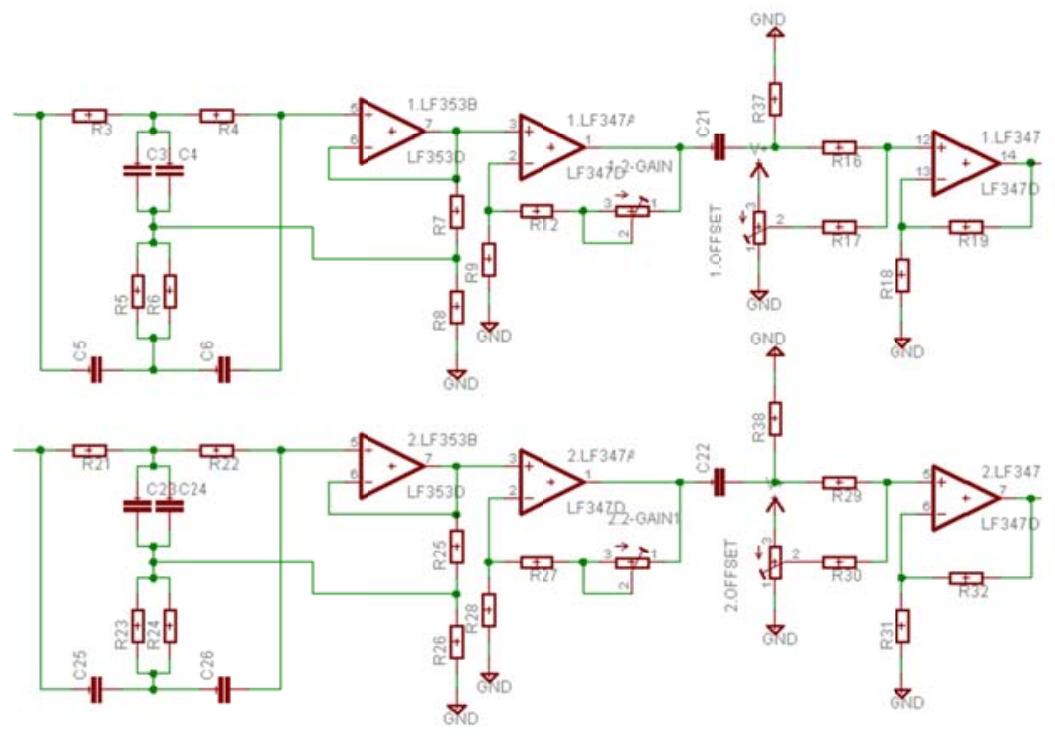




\section{Apêndice E - Esquema do circuito de condicionamento de biopotenciais - Parte 3}

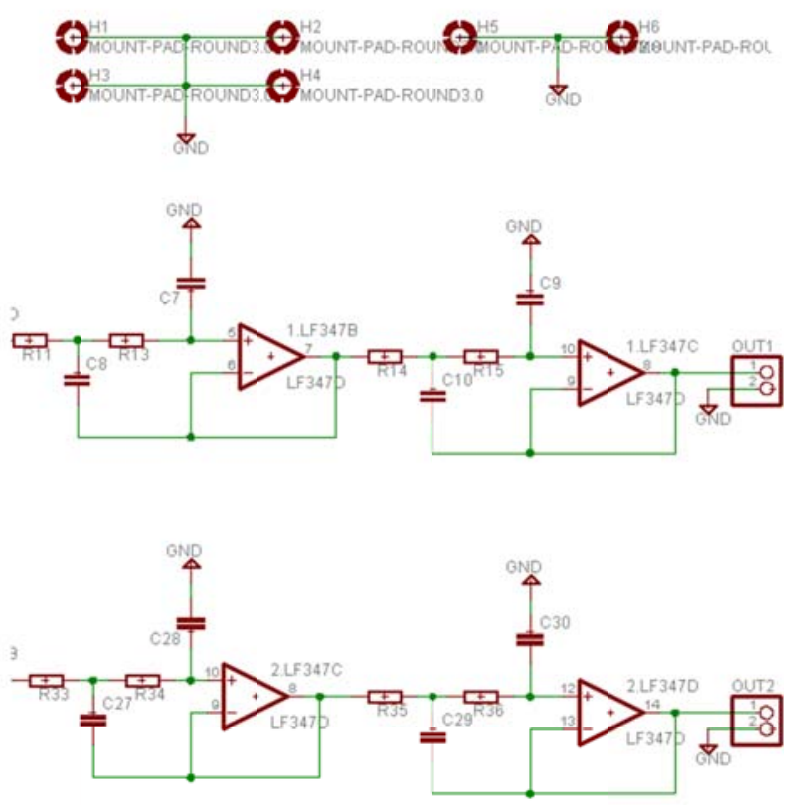




\section{Apêndice F - Caracterização do acelerômetro MX2125 - Eixo X}

Tabela A.1 - Caracterização do eixo x do acelerômetro MX2125.

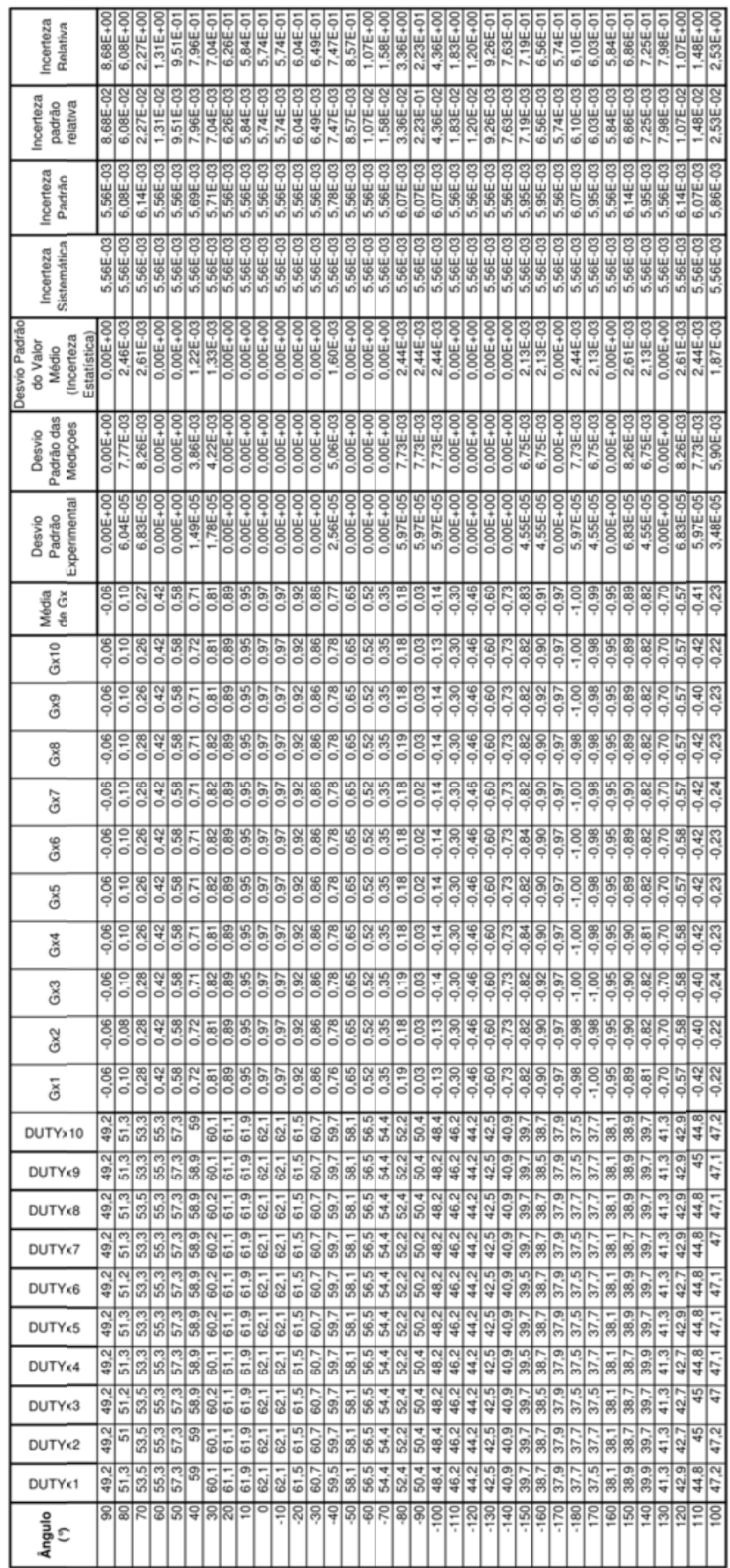




\section{Apêndice G - Caracterização do acelerômetro MX2125 - Eixo Y}

Tabela A.2 - Caracterização do eixo y do acelerômetro MX2125.

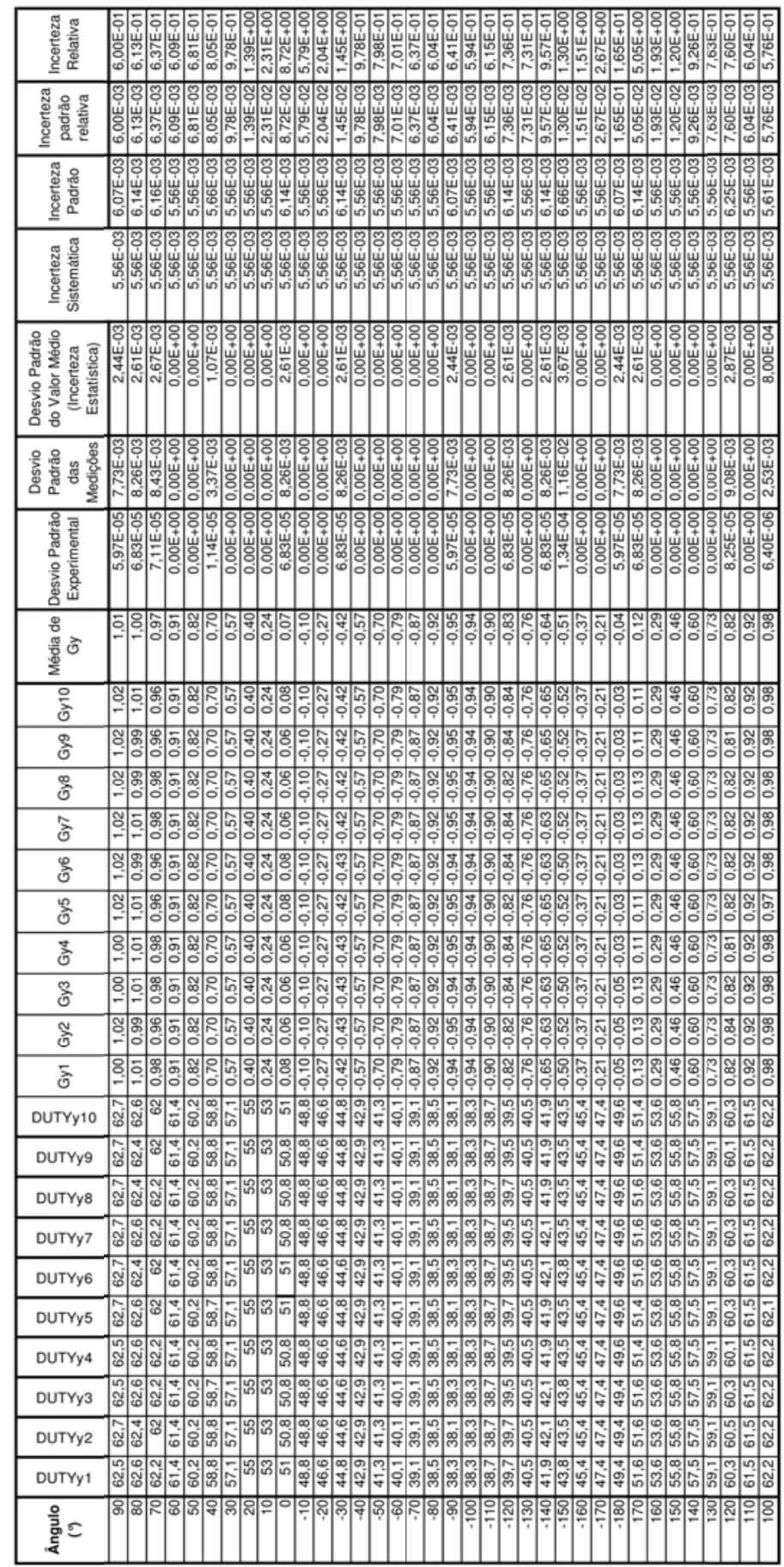




\section{Apêndice H - Caracterização do acelerômetro MX2125.}

Tabela A.3 - Caracterização do acelerômetro MX2125.

\begin{tabular}{|c|c|c|c|c|c|c|c|c|}
\hline $\begin{array}{c}\text { Ângulo } \\
\left({ }^{\circ}\right)\end{array}$ & Média de Gx & $\begin{array}{c}\text { Incerteza } \\
\text { Padrão de } \\
\text { Gx } \\
\end{array}$ & $\begin{array}{l}\text { Média de } \\
\text { Gy }\end{array}$ & $\begin{array}{c}\text { Incerteza } \\
\text { Padrão de } \\
\text { Gy } \\
\end{array}$ & $\begin{array}{c}\text { Incerteza do } \\
\text { Ângulo } \\
\text { medido }\end{array}$ & $\begin{array}{c}\text { Ângulo } \\
\text { medido } \\
(9)\end{array}$ & $\begin{array}{c}\text { Erro } \\
\text { Absoluto }\end{array}$ & \begin{tabular}{|c|} 
Erro \\
Relativo \\
$(\%)$ \\
\end{tabular} \\
\hline 90 & $-0,06$ & $1,01 \mathrm{E}+00$ & 1,01 & $1,43 \mathrm{E}+00$ & $5,00 \mathrm{E}-01$ & 93,6 & 3,6 & 0,04 \\
\hline 80 & 0,10 & $1,01 \mathrm{E}+00$ & 1,00 & $1,42 \mathrm{E}+00$ & $5,00 \mathrm{E}-01$ & 84,3 & 4,3 & 0,05 \\
\hline 70 & 0,27 & $1,01 \mathrm{E}+00$ & 0,97 & $1,40 \mathrm{E}+00$ & $5,00 \mathrm{E}-01$ & 74,4 & 4,4 & 0,06 \\
\hline 60 & 0,42 & $1,01 \mathrm{E}+00$ & 0,91 & $1,36 \mathrm{E}+00$ & $5,01 \mathrm{E}-01$ & 65,1 & 5,1 & 0,08 \\
\hline 50 & 0,58 & $1,00 \mathrm{E}+00$ & 0,82 & $1,29 \mathrm{E}+00$ & $5,01 \mathrm{E}-01$ & 54,4 & 4,4 & 0,08 \\
\hline 40 & 0,71 & $1,00 \mathrm{E}+00$ & 0,70 & $1,22 \mathrm{E}+00$ & $4,91 \mathrm{E}-01$ & 44,5 & 4,5 & 0,10 \\
\hline 30 & 0,81 & $9,91 \mathrm{E}-01$ & 0,57 & $1,14 \mathrm{E}+00$ & $4,60 \mathrm{E}-01$ & 35,0 & 5,0 & 0,14 \\
\hline 20 & 0,89 & $9,74 \mathrm{E}-01$ & 0,40 & $1,05 \mathrm{E}+00$ & $3,77 \mathrm{E}-01$ & 24,2 & 4,2 & 0,18 \\
\hline 10 & 0,95 & $9,82 \mathrm{E}-01$ & 0,24 & $1,01 \mathrm{E}+00$ & $2,44 \mathrm{E}-01$ & 14,1 & 4,1 & 0,29 \\
\hline 0 & 0,97 & $9,71 \mathrm{E}-01$ & 0,07 & $9,73 \mathrm{E}-01$ & $7,33 \mathrm{E}-02$ & 4,2 & 4,2 & 1,00 \\
\hline-10 & 0,97 & $9,73 \mathrm{E}-01$ & $-0,10$ & 9,77E-01 & $1,00 \mathrm{E}-01$ & $-5,7$ & 4,3 & 0,77 \\
\hline-20 & 0,92 & $9,59 \mathrm{E}-01$ & $-0,27$ & 9,97E-01 & $2,78 \mathrm{E}-01$ & $-16,5$ & 3,5 & 0,21 \\
\hline-30 & 0,86 & $9,55 \mathrm{E}-01$ & $-0,42$ & $1,04 \mathrm{E}+00$ & $3,96 \mathrm{E}-01$ & $-26,3$ & 3,7 & 0,14 \\
\hline-40 & 0,77 & $9,60 \mathrm{E}-01$ & $-0,57$ & $1,12 \mathrm{E}+00$ & $4,65 \mathrm{E}-01$ & $-36,3$ & 3,7 & 0,10 \\
\hline-50 & 0,65 & $9,51 \mathrm{E}-01$ & $-0,70$ & $1,18 \mathrm{E}+00$ & 4,97E-01 & $-47,0$ & 3,0 & 0,06 \\
\hline-60 & 0,52 & 9,47E-01 & $-0,79$ & $1,23 E+00$ & $5,05 \mathrm{E}-01$ & $-56,7$ & 3,3 & 0,06 \\
\hline-70 & 0,35 & $9,40 \mathrm{E}-01$ & $-0,87$ & $1,28 \mathrm{E}+00$ & $5,04 \mathrm{E}-01$ & $-68,0$ & 2,0 & 0,03 \\
\hline-80 & 0,18 & $9,38 \mathrm{E}-01$ & $-0,92$ & $1,31 \mathrm{E}+00$ & $5,02 \mathrm{E}-01$ & $-78,9$ & 1,1 & 0,01 \\
\hline-90 & 0,03 & $9,48 \mathrm{E}-01$ & $-0,95$ & $1,34 \mathrm{E}+00$ & $5,01 \mathrm{E}-01$ & $-88,4$ & 1,6 & 0,02 \\
\hline-100 & $-0,14$ & $9,46 \mathrm{E}-01$ & $-0,94$ & $1,33 \mathrm{E}+00$ & 5,02E-01 & $-98,5$ & 1,5 & 0,02 \\
\hline-110 & $-0,30$ & $9,54 \mathrm{E}-01$ & $-0,90$ & $1,31 \mathrm{E}+00$ & $5,03 \mathrm{E}-01$ & $-108,6$ & 1,4 & 0,01 \\
\hline-120 & $-0,46$ & $9,54 \mathrm{E}-01$ & $-0,83$ & $1,27 \mathrm{E}+00$ & $5,05 \mathrm{E}-01$ & $-119,1$ & 0,9 & 0,01 \\
\hline-130 & $-0,60$ & $9,68 \mathrm{E}-01$ & $-0,76$ & $1,23 E+00$ & $5,01 \mathrm{E}-01$ & $-128,3$ & 1,7 & 0,01 \\
\hline-140 & $-0,73$ & $9,70 \mathrm{E}-01$ & $-0,64$ & $1,16 \mathrm{E}+00$ & $4,85 \mathrm{E}-01$ & $-138,6$ & 1,4 & 0,01 \\
\hline-150 & $-0,83$ & $9,73 \mathrm{E}-01$ & $-0,51$ & $1,10 \mathrm{E}+00$ & $4,41 \mathrm{E}-01$ & $-148,2$ & 1,8 & 0,01 \\
\hline-160 & $-0,91$ & $9,79 \mathrm{E}-01$ & $-0,37$ & $1,05 \mathrm{E}+00$ & $3,53 \mathrm{E}-01$ & $-157,9$ & 2,1 & 0,01 \\
\hline-170 & $-0,97$ & $9,90 \mathrm{E}-01$ & $-0,21$ & $1,01 \mathrm{E}+00$ & $2,12 \mathrm{E}-01$ & $-167,9$ & 2,1 & 0,01 \\
\hline-180 & $-1,00$ & 9,96E-01 & $-0,04$ & 9,97E-01 & $3,72 \mathrm{E}-02$ & $-177,9$ & 2,1 & 0,01 \\
\hline 170 & $-0,99$ & $9,95 \mathrm{E}-01$ & 0,12 & $1,00 \mathrm{E}+00$ & $1,24 \mathrm{E}-01$ & 173,0 & 3,0 & 0,02 \\
\hline 160 & $-0,95$ & $9,95 \mathrm{E}-01$ & 0,29 & $1,04 \mathrm{E}+00$ & $2,85 \mathrm{E}-01$ & 163,2 & 3,2 & 0,02 \\
\hline 150 & $-0,89$ & $1,01 \mathrm{E}+00$ & 0,46 & $1,11 \mathrm{E}+00$ & 4,09E-01 & 152,6 & 2,6 & 0,02 \\
\hline 140 & $-0,82$ & $1,02 \mathrm{E}+00$ & 0,60 & $1,18 \mathrm{E}+00$ & $4,65 \mathrm{E}-01$ & 143,8 & 3,8 & 0,03 \\
\hline 130 & $-0,70$ & $1,01 \mathrm{E}+00$ & 0,73 & $1,24 \mathrm{E}+00$ & 4,94E-01 & 133,7 & 3,7 & 0,03 \\
\hline 120 & $-0,57$ & $1,00 \mathrm{E}+00$ & 0,82 & $1,30 \mathrm{E}+00$ & $5,01 \mathrm{E}-01$ & 124,9 & 4,9 & 0,04 \\
\hline 110 & $-0,41$ & $1,01 \mathrm{E}+00$ & 0,92 & $1,36 \mathrm{E}+00$ & $5,01 \mathrm{E}-01$ & 114,1 & 4,1 & 0,04 \\
\hline 100 & $-0,23$ & $1,00 \mathrm{E}+00$ & 0,98 & $1,40 \mathrm{E}+00$ & $5,00 \mathrm{E}-01$ & 103,3 & 3,3 & 0,03 \\
\hline
\end{tabular}




\section{Apêndice I - Caracterização do MX2125 para medida de Amplitude de Movimento}

Tabela A.4 - Caracterização do MX2125 para medida de ADM.

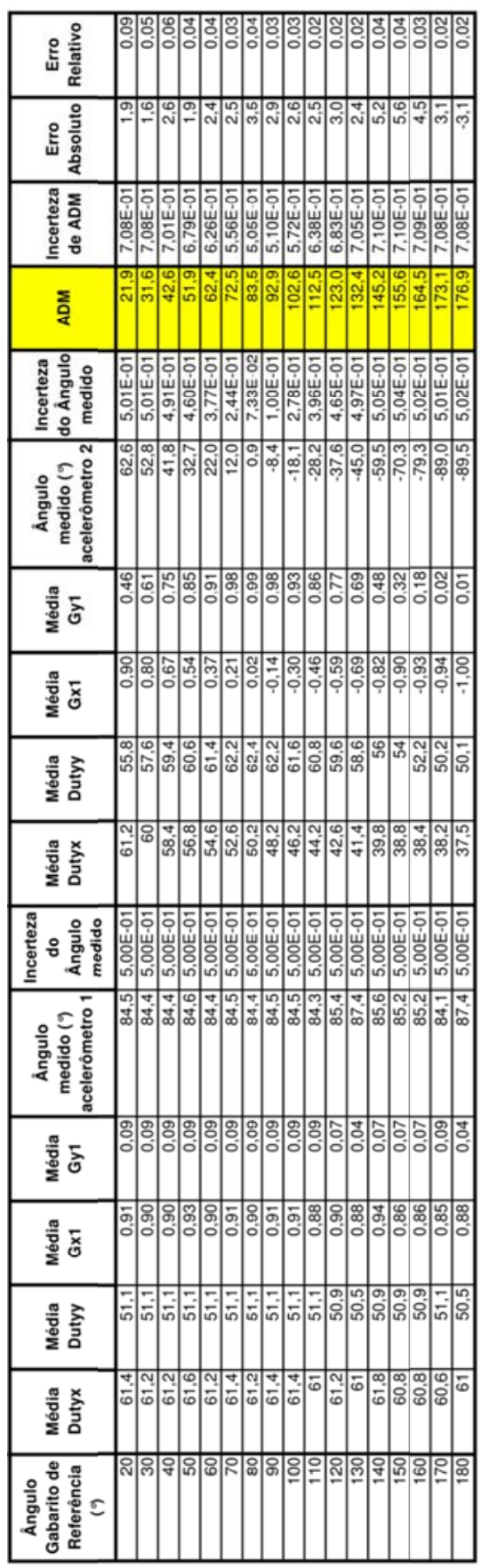




\section{Apêndice J - Esquema de polarização do microcontrolador ATmega8535}

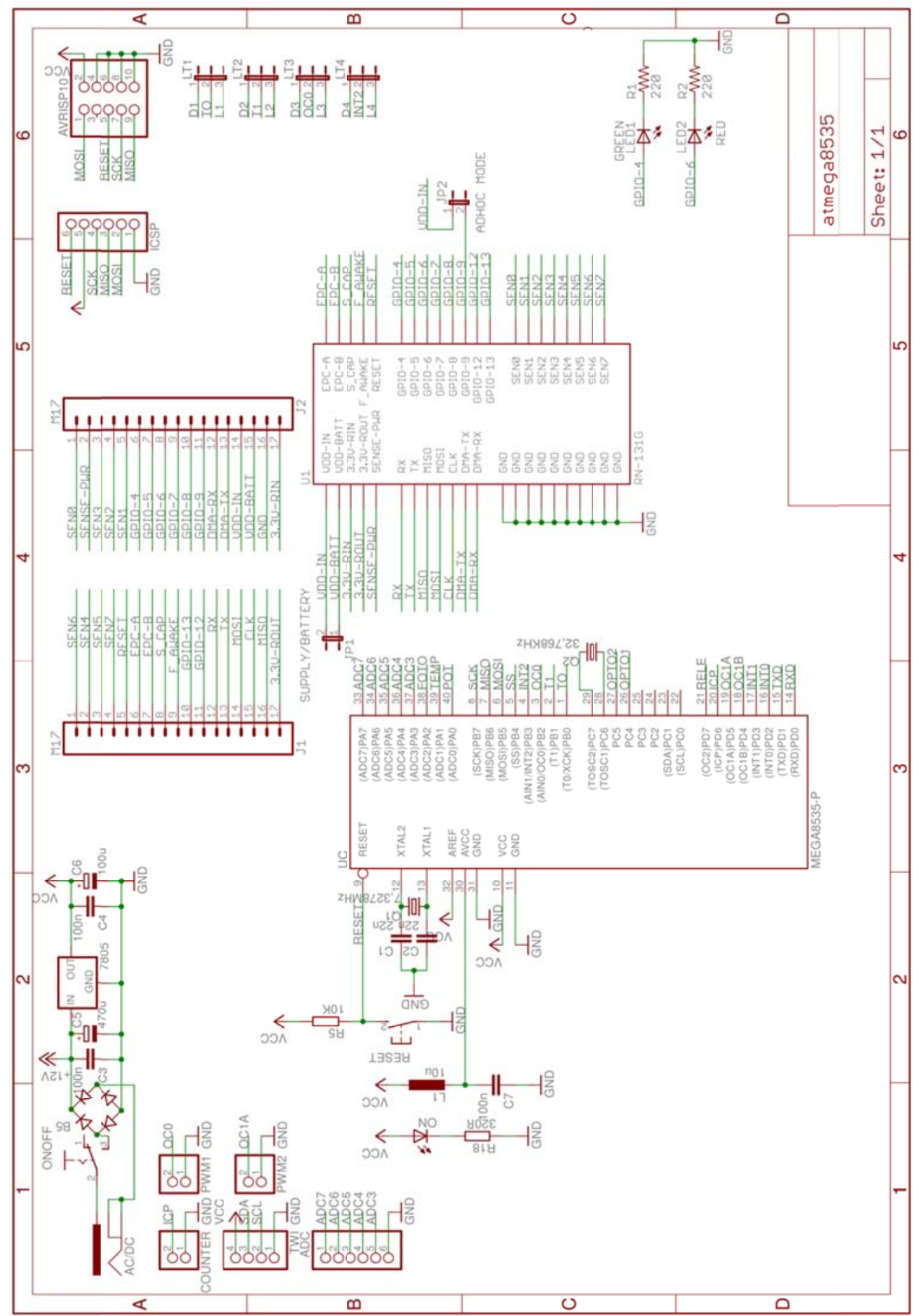




\section{Apêndice K - Esquema de polarização do microcontrolador AT90USB162.}

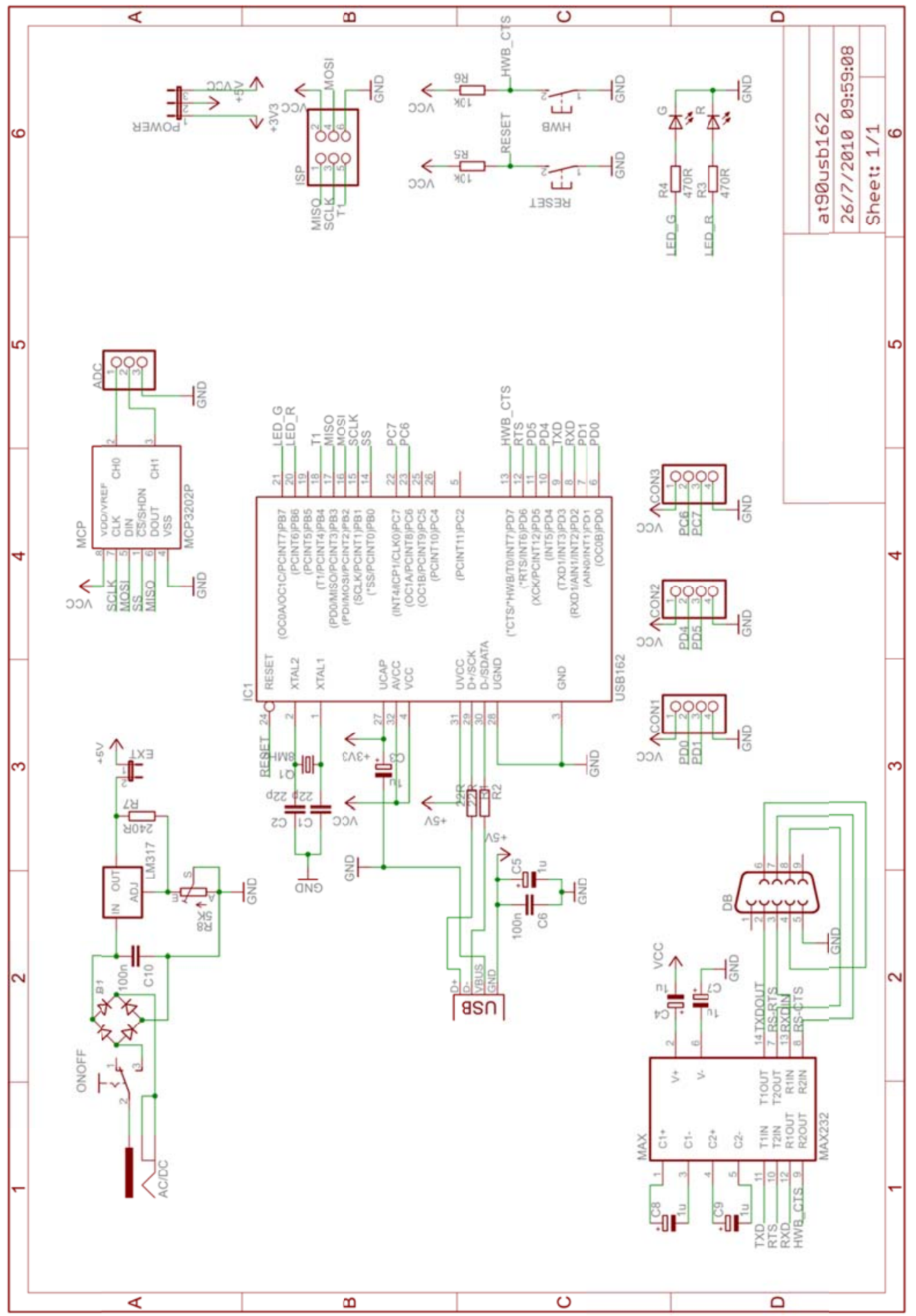




\section{Anexo A - Microcontrolador ATMega8535}

\section{Features}

- High-performance, Low-power AVR ${ }^{\circ}$-bit Microcontroller

- Advanced RISC Architecture

- 130 Powerful Instructions - Most Single Clock Cycle Execution

- $32 \times 8$ General Purpose Working Registers

- Fully Static Operation

- Up to 16 MIPS Throughput at $16 \mathrm{MHz}$

- On-chip 2-cycle Multiplier

- Nonvolatile Program and Data Memories

- 8K Bytes of In-System Selt-Programmable Flash

Endurance: 10,000 Write/Erase Cycles

- Optional Boot Code Section with Independent Lock Bits

In-System Programming by On-chip Boot Program

True Read-While-Write Operation

- 512 Bytes EEPROM

Endurance: 100,000 Write/Erase Cycles

- 512 Bytes Internal SRAM

- Programming Lock for Software Security

- Peripheral Features

- Two 8-bit Timer/Counters with Separate Prescalers and Compare Modes

- One 16-bit Timer/Counter with Separate Prescaler, Compare Mode, and Capture Mode

- Real Time Counter with Separate Oscillator

- Four PWM Channels

- 8-channel, 10-bit ADC

8 Single-ended Channels

7 Differential Channels for TQFP Package Only

2 Differential Channels with Programmable Gain at 1x, 10x, or 200x for TQFP Package Only

- Byte-oriented Two-wire Serial Interface

- Programmable Serial USART

- Programmable Serial USART

- Programmable Watchdog Timer with Separate On-chip Oscillator

- On-chip Analog Comparator

- Special Microcontroller Features

- Power-on Reset and Programmable Brown-out Detection

- Internal Calibrated RC Oscillator

- External and Internal Interrupt Sources

- Six Sleep Modes: Idle, ADC Noise Reduction, Power-save, Power-down, Standby and Extended Standby

- vo and Packages

- 32 Programmable Vo Lines

- 40-pin PDIP, 44-lead TQFP, 44-lead PLCC, and 44-pad OFNMLLF

- Operating Voltages

$-2.7-5.5 \mathrm{~V}$ for ATmega8535L

- 4.5 - $5.5 \mathrm{~V}$ for ATmega8535

- Speed Grades

- $0.8 \mathrm{MHz}$ for ATmege8535L

- $0.16 \mathrm{MHz}$ for ATmega8535

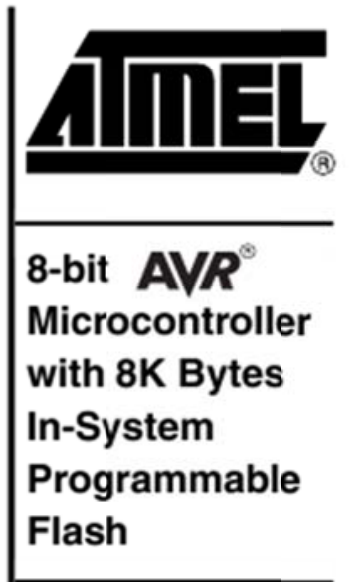

ATmega8535

ATmega8535L

\section{A且目}




\section{Anexo B - Módulo Wifi WiFly GSX 802.11b/g}

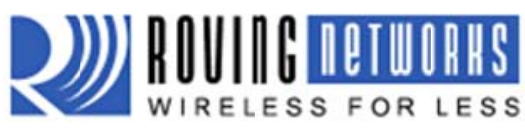

www.rovingnetworks.com

\section{WiFly GSX $802.11 \mathrm{~b} / \mathrm{g}$ Wireless LAN Module}

\section{Features}

- Qualified $2.4 \mathrm{GHz}$ IEEE $802.11 \mathrm{~b} / \mathrm{g}$ transceiver

- High throughput, $1 \mathrm{Mbps}$ sustained data rate with TCP/IP and WPA2

- Ultra-low power - 4uA sleep, 40mA Rx. $210 \mathrm{~mA}$ TX (max)

- Small, compact surface mount module

- On board ceramic chip antenna and U.FL connector for external antenna

- 8 Mbit flash memory and 128 KB RAM

- UART hardware interface

- 10 general purpose digital L/O

- 8 analog sensor interfaces

- Real-time clock for wakeup and time stamping

- Accepts $3.3 \mathrm{~V}$ regulated or 2-3V battery

- Supports Adhoc connections

- On board ECOS -OS, TCP/P stacks

- Wi-Fi Alliance certified for WPA2-PSK

- FCC/CE/ICS certified and RoHS compliant.

- Industrial (RN-131G) and commercial (RN-131C) grade temperature options

\section{Applications}

- Remote equipment monitoring

- Telemetry

- Industrial sensors and controls On-board chip antenna

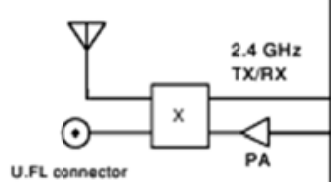
U.FL connector For optional extomal antenna

\section{RN-131G \& RN-131C}

RN-131-DS v2.5 5/11/2010

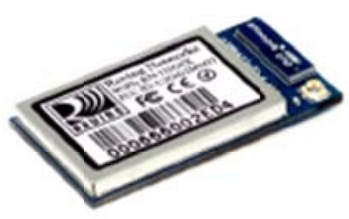

- Home Automation

- Medical device monitoring

Description

The WiFly GSX module is a stand alone, embedded wireless 802.11 networking module. Because of its small form factor and extremely low power consumption, the RN-131G is perfect for mobile wireless applications such as asset monitoring. GPS tracking and battery sensors. The WiFly GSX module incorporates a $2.4 \mathrm{GHz}$ radio, processor, TCP/P stack, real-time clock, crypto accelerator, power

management and analog sensor interfaces. This complete solution is preloaded with software to simplify integration and minimizes development of your application. In the simplest configuration the hardware only requires four connections (PWR, TX, $\mathrm{RX}, \mathrm{GND}$ ) to create a wireless data connection. Additionally, the sensor interface provides

temperature, audio, motion, acceleration and other analog data without requiring additional hardware. The Wifly GSX module is programmed and controlled with a simple ASCII command language. Once the Wifly GSX is setup it can scan to find an access point. associate, authenticate and connect over any Witt network.

Block Diagram
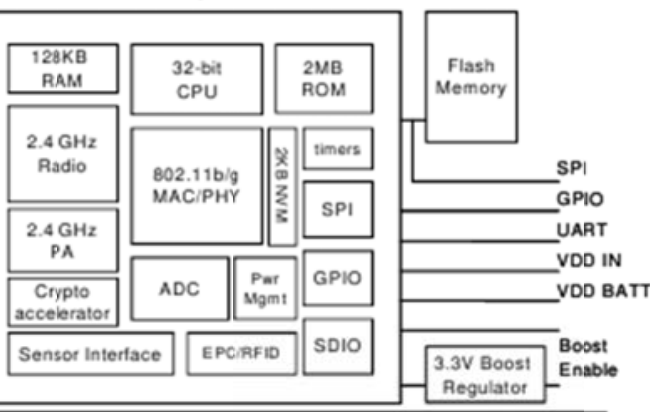

809 University Avenue - Los Gatos, CA $95032 \cdot$ Tel (408) 395-6539 - info@RovingNetworks.com

$\sim$ page $1 \sim$ 


\section{Anexo C - Microcontrolador AT90USB162}

\section{Features}

- High Performance, Low Power AVR" 8-Bit Microcontroller

- Advanced RISC Architecture

- 125 Powertul Instructions - Most Single Clock Cycle Execution

- $32 \times 8$ General Purpose Working Registers

- Fully Static Operation

- Up to 16 MIPS Throughput at $16 \mathrm{MHz}$

- Non-volatile Program and Data Memories

- 8K/16K Bytes of In-System Selt-Programmable Flash - Endurance: 10,000 Write/Erase Cycles

- Optional Boot Code Section with Independent Lock Bits

- USB boot-loader programmed by default in the factory

- In-System Programming by on-chip Boot Program hardware-activated after reset

- True Read-While-Write Operation

- 512 Bytes EEPROM

- Endurance: 100,000 Write/Erase Cycles

- 512 Bytes Internal SRAM

- Programming Lock for Software Security

- USB 2.0 Full-speed Device Module with Interrupt on Transter Completion

- Complies fully with Universal Serial Bus Specification REV 2.0

- $48 \mathrm{MHz}$ PLL for Full-speed Bus Operation : data transfer rates at $12 \mathrm{Mbit} / \mathrm{s}$

- Fully independant 176 bytes USB DPRAM for endpoint memory allocation

- Endpoint 0 for Control Transfers: from 8 up to 64-bytes

- 4 Programmable Endpoints:

- IN or Out Directions

- Bulk, Interrupt and lsochronous Transfers

- Programmable maximum packet size from 8 to 64 bytes

- Programmable single or double butter

- Suspend/Resume Interrupts

- Microcontroller reset on USB Bus Reset without detach

- USB Bus Disconnection on Microcontroller Request

- USB pad multiplexed with PS/2 peripheral for single cable capability

- Peripheral Features

- PS/2 compliant pad

- One 8-bit Timer/Counters with Separate Prescaler and Compare Mode (two 8-bit PWM channels)

- One 16-bit Timer/Counter with Separate Prescaler, Compare and Capture Mode (three 8-bit PWM channels)

- USART with SPI master only mode and hardware flow control (RTS/CTS)

- Master/Slave SPI Serial Interface

- Programmable Watchdog Timer with Separate On-chip Oscillator

- On-chip Analog Comparator

- Interrupt and Wake-up on Pin Change

- On Chip Debug Interface (debugWIRE)

- Special Microcontroller Features

- Power-On Reset and Programmable Brown-out Detection

- Internal Calibrated Oscillator

- External and Internal Interrupt Sources

\begin{tabular}{|l}
\hline 8-bit AYR \\
Microcontroller \\
with \\
8/16K Bytes of \\
ISP Flash \\
and USB \\
Controller
\end{tabular}

AT90USB82

AT90USB162

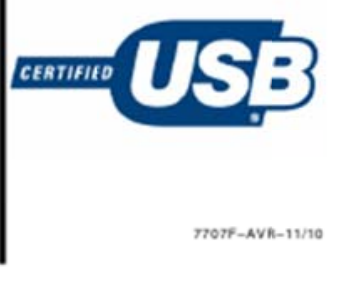




\section{Anexo D - Acelerômetro Parallax MX2125}

\begin{tabular}{|c|c|c|}
\hline 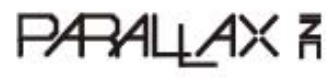 & 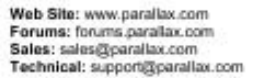 & $\begin{array}{l}\text { Ottice: (1916) 624-8333 } \\
\text { Fax: (196) 624-8003 } \\
\text { Sales: (B83) 512-1024 } \\
\text { Tech support: (888) 997-8267 }\end{array}$ \\
\hline
\end{tabular}

\section{Memsic 2125 Dual-Axis Accelerometer (\#28017)}

The Memsic 2125 is a low-cost thermal accelerometer capable of measuring tilt, collision, static and dynamic acceleration, rotation, and vibration with a range of $\pm 3 \mathrm{~g}$ on two axes. Memsic provides the 2125 IC in a surface-mount format. Parallax mounts the circuit on a tiny PCB providing all I/O connections so it can easily be inserted on a breadboard or through-hole prototype area.

\section{Features}

- Measures $\pm 3 \mathrm{~g}$ on each axis

- Simple pulse output of $g$-force for each axis

- Convenient 6-pin 0.1" spacing DIP module

- Analog output of temperature (TOut pin)

- Fully temperature compensated over 0 to $70^{\circ} \mathrm{C}$ operating temperature range

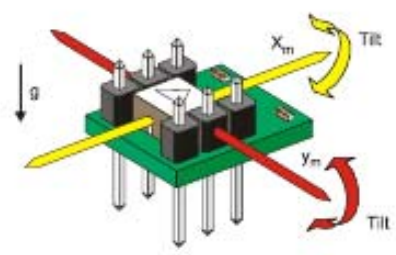

\section{Key Specifications}

- Power Requirements: 3.3 to 5 VDC $<5$ ma supply current

- Communication: TTL/CMOS compatible $100 \mathrm{~Hz}$ PWM output signal with duty cycle proportional to acceleration

- Dimensions: $0.42 \times 0.42 \times 0.45$ in $(10.7 \times 10.7 \times 11.8 \mathrm{~mm})$

- Operating temperature: 32 to $158^{\circ} \mathrm{F}$ ( 0 to $\left.70^{\circ} \mathrm{C}\right)$

\section{Application Ideas}

- Dual-axis tilt and acceleration sensing for autonomous robot navigation

- R/C tilt controller or autopilot

- Tilt-sensing Human Interface Device

- Motion/lack-of-motion sensor for alarm system

- Single-axis rotational angle and position sensing

\section{Theory of Operation}

The MX2125 has a chamber of gas with a heating element in the center and four temperature sensors around its edge. When the accelerometer is level, the hot gas pocket rises to the top-center of the chamber, and all the sensors will measure the same temperature.
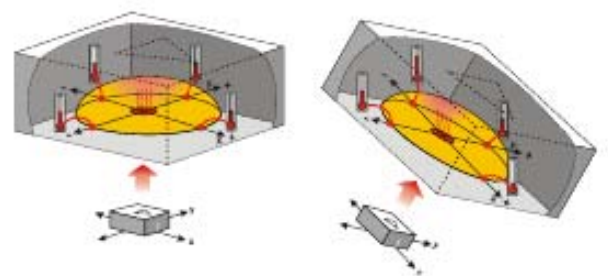

By tilting the accelerometer, the hot gas will collect closer to some of temperature sensors. By comparing the sensor temperatures, both static acceleration (gravity and tilt) and dynamic acceleration (like taking a ride in a car) can be detected. The MX2125 converts the temperature measurements into signals (pulse durations) that are easy for microcontrollers to measure and decipher. 


\section{Anexo E - Meta TEDS - IEEE1451.0}

Tabela A.5 - Meta TEDS IEEE1451.0 (IEEE, 2007a).

\begin{tabular}{|c|c|c|c|c|}
\hline $\begin{array}{l}\text { Field } \\
\text { type }\end{array}$ & $\begin{array}{l}\text { Field } \\
\text { name }\end{array}$ & Description & Data type & Woctets \\
\hline - & & Length & Ulnt32 & 4 \\
\hline $0-2$ & - & Reserved & - & - \\
\hline 3 & TEDSID & TEDS Identification Header & Ulnt8 & 4 \\
\hline 4 & UUID & Globally Unique Identifier & UUID & 10 \\
\hline $5-9$ & - & Reserved & - & - \\
\hline \multicolumn{5}{|c|}{ Timing-related information } \\
\hline 10 & OholdOff & Operational time-out & Float32 & 4 \\
\hline 11 & SHoldOff & Slow-access time-out & Float32 & 4 \\
\hline 12 & Test Time & Self-Test Time & Float32 & 4 \\
\hline \multicolumn{5}{|c|}{ Number of implemented TransducerChannels } \\
\hline 13 & MaxChan & Number of implemented TransducerChannels & Ulnt 16 & 2 \\
\hline 14 & CGroup & ControlGroup information sub-block & - & - \\
\hline \multicolumn{5}{|c|}{ Types 20, and 21 define one ControlGiroup. } \\
\hline 20 & GirpType & ControlGroup type & Ulnt8 & 1 \\
\hline 21 & MemL.ist & ControlGroup member list & $\begin{array}{ll}\text { array } \\
\text { Ulnt16 }\end{array}$ & NTe \\
\hline 15 & VGroup & NectorGroup information sub-block & - & - \\
\hline \multicolumn{5}{|c|}{ Types 20 and 21 define one VectorGroup. } \\
\hline 20 & GrpType & VectorGroup type & Ulnt8 & 1 \\
\hline 21 & MemL_ist & VectorGroup member list & $\begin{array}{l}\text { amay } \\
\text { Ulnt16 }\end{array}$ & NTV \\
\hline 16 & GeoLoc & Specialized VectorGroup for geographic location & - & - \\
\hline \multicolumn{5}{|c|}{ Types 24,20 , and 21 define one set of geographic location information. } \\
\hline 24 & LocEnum & $\begin{array}{l}\text { An enumeration defining how location information is } \\
\text { provided }\end{array}$ & Ulnt8 & 1 \\
\hline 20 & GipType & VectorGroup type & Ulnt8 & 1 \\
\hline 21 & Meml_ist & VectorGroup member list & $\begin{array}{l}\text { amay } \\
\text { Ulnt16 }\end{array}$ & NTr \\
\hline 17 & Proxies & TransducerChannel proxy definition sub-block & - & - \\
\hline \multicolumn{5}{|c|}{ Types 22,23 , and 21 define one TransducerChannel proxy. } \\
\hline 22 & ChanNum & TransducerChannel number of the TransducerChannel proxy & Ulnt 16 & 1 \\
\hline 23 & Organiz & TransducerChannel proxy data-set organization & Utnt8 & 1 \\
\hline 21 & MemL ist & TransducerChannel proxy member list & $\begin{array}{l}\text { amay } \\
\text { Ulnt16 }\end{array}$ & NTp \\
\hline $18-19$ & - & Reserved & - & - \\
\hline $25-127$ & - & Resenved & - & - \\
\hline $128-255$ & - & Open to manufacturers & - & - \\
\hline- & & Checksum & Ulnt16 & 2 \\
\hline
\end{tabular}




\section{Anexo F - TransducerChannel TEDS - IEEE1451.0}

Tabela A.6 - TransducerChannel TEDS IEEE1451.0 (IEEE, 2007a).

\begin{tabular}{|c|c|c|c|c|}
\hline Field & Field name & Description & Type & 8 octets \\
\hline- & & TEDS length & UInt32 & 4 \\
\hline $0-2$ & - & Reserved & - & - \\
\hline 3 & TEDSID & TEDS Identification & UInt8 & 4 \\
\hline 4.9 & - & Reserved & - & - \\
\hline \multicolumn{5}{|c|}{ TransducerChannel related information } \\
\hline 10 & CalKey & Calibration key & UInt8 & 1 \\
\hline 11 & ChanType & TransducerChannel type key & UInt8 & 1 \\
\hline 12 & PhyUnits & Physical Units & UNITS & 11 \\
\hline 50 & UnitType & Physical Units interpretation enumeration & UInt8 & 1 \\
\hline S1 & Radians & The exponent for Radians & UInt8 & 1 \\
\hline 52 & SterRad & The exponent for Steradians & UInt8 & 1 \\
\hline 53 & Meters & The exponent for Meters & UInt8 & $\mathrm{T}$ \\
\hline 54 & Kilogram & The exponent for Kilograms & UInt8 & 1 \\
\hline 55 & Seconds & The exponent for Seconds & UInt8 & 1 \\
\hline 56 & Amperes & The exponent for Amperes & UInt8 & 1 \\
\hline 57 & Kelvins & The exponent for Kelvins & UInt8 & 1 \\
\hline 58 & Moles & The exponent for Moles & UInt8 & 1 \\
\hline 59 & Candelas & The exponent for Candelas & UInt8 & 1 \\
\hline 60 & UnitsExt & TEDS access code for units extension & UInt8 & 1 \\
\hline 13 & LowLimit & Design operational lower range limit & Float32 & 4 \\
\hline 14 & HiL_imit & Design operational upper range limit & Float32 & 4 \\
\hline 15 & OError & Worst-case uncertainty & Float32 & 4 \\
\hline 16 & SelfTest & Self-test key & UInt8 & 1 \\
\hline 17 & MRange & Multi-range capability & UInt8 & 1 \\
\hline - & & Data converter-related information & - & - \\
\hline 18 & Sample & & - & - \\
\hline 40 & DatModal & Data model & UInt8 & 1 \\
\hline 41 & Modt.enth & Data model length & UInt8 & 1 \\
\hline 42 & SigBits & Model significant bits & UInt16 & 2 \\
\hline 19 & DataSet & & & \\
\hline 43 & Repeats & Maximum data repetitions & UInt16 & 2 \\
\hline 44 & SOrigin & Series origin & Float32 & 4 \\
\hline 45 & StepSize & Series increment & Float32 & 4 \\
\hline 46 & SUnits & Series units & UNITS & 11 \\
\hline 47 & PreTrigg & Maximum pte-trigger samples & UInt 16 & 2 \\
\hline \multicolumn{5}{|c|}{ Timing-related information } \\
\hline 20 & UpdateT & TransducerChannel update time ( $\left.\mathrm{t}_{2}\right)$ & Float32 & 4 \\
\hline 21 & WSetupT & TransducerChannel write setup time ( $\left.\mathrm{ks}_{\mathrm{s}}\right)$ & Float32 & 4 \\
\hline 22 & RSetupT & TransducerChannel read setup time $\left(\mathrm{t}_{\mathrm{n}}\right)$ & Float32 & 4 \\
\hline 23 & SPeriod & TransducerChannel sampling period $\left(t_{0}\right)$ & Float32 & 4 \\
\hline 24 & WarmU $\mathrm{T}$ & TransducerChannel warm-up time & Float32 & 4 \\
\hline 29 & RDelayT & TransducerChannel read delay time (ta) & Float32 & 4 \\
\hline 26 & TestTime & TransducerChannel self-test time requirement & Float32 & 4 \\
\hline \multicolumn{5}{|c|}{ Tume of the sample information } \\
\hline 27 & TimeSre & Source for the time of sample & UInt8 & 1 \\
\hline 28 & InPropDI & Incoming propagation delay through the data transport logic & Float32 & 4 \\
\hline 29 & OutPropD & Outgoing propagation delay through the data transport logic & Float32 & 4 \\
\hline 30 & TSError & Trigger-to-sample delay uncertainty & Float32 & 4 \\
\hline \multicolumn{5}{|c|}{ Aitributes } \\
\hline 31 & Sampling & Sampling attribute & - & - \\
\hline 48 & SampMode & Sampling mode capability & UInt8 & 1 \\
\hline 49 & SDefault & Default sampling mode & UInt8 & 1 \\
\hline 32 & DataXmit & Data transmission attribute & UInt8 & 1 \\
\hline 33 & Buffered & Buffered attribute & UInt8 & 1 \\
\hline 34 & EndOtSet & End-of-data-set operation attnbute & UInt8 & 1 \\
\hline 35 & EdgeRpt & Edge-to-report attribute & Ulnt8 & 1 \\
\hline 36 & Actllalt & Actuator-halt attribute & UInt8 & 1 \\
\hline
\end{tabular}

Table 48--Structure of the TransducerChannel TEDS data block (continued)

\begin{tabular}{|c|c|c|c|c|}
\hline Ficld & Field name & Description & Type & 8 octets \\
\hline \multicolumn{5}{|c|}{ Sensitivity } \\
\hline 37 & Directon & Sensitivity direction & Float32 & 4 \\
\hline 38 & DAngles & Direction angles & \begin{tabular}{|l|} 
Two \\
Float32
\end{tabular} & 8 \\
\hline \multicolumn{5}{|l|}{ Options } \\
\hline 39 & ESOption & Event sensor options & UInt8 & 1 \\
\hline $61-127$ & - & Reserved & - & - \\
\hline $128-255$ & - & Open to manufacturers & - & - \\
\hline- & & Checksum & UInt 16 & 2 \\
\hline
\end{tabular}




\section{Anexo G - User's Transducer Name TEDS - IEEE1451.0}

Tabela A.7 - User's Transducer Name TEDS IEEE1451.0 (IEEE, 2007a).

\begin{tabular}{|c|c|c|c|c|}
\hline Field & Description & Type & Type & 8 octets \\
\hline- & TEDS length & UInt32 & UInt32 & 4 \\
\hline $0-2$ & - & Reserved & - & - \\
\hline 3 & TEDSID & TEDS identification header & Ulnt8 & Ulnt8 \\
\hline 4-9 & - & Reserved & - & - \\
\hline 10 & EndUserData & Variable & & Variable \\
\hline - & Checksum & Uln:16 & & 2 \\
\hline
\end{tabular}




\section{Anexo H - PHY TEDS - IEEE1451.2}

Tabela A.8 - PHY TEDS IEEE1451.2 extraído de Song e Lee (2008b).

\begin{tabular}{|c|c|c|c|c|c|}
\hline te & & & & & - $\mathrm{a} \underline{\mathrm{x}}$ \\
\hline \multicolumn{6}{|c|}{ PhyTEDS } \\
\hline \multicolumn{2}{|c|}{ Field type Field Name I } & Description & \multicolumn{3}{|c|}{ Data Type Number of Octets Length Value } \\
\hline 3 & TEDSID & TFDS Identification Header & UInts & 4 & 2 D01: 11521 \\
\hline 10 & RS232 & IREE 1451.2-RS232 Physical Type & UInts & 1 & $1: 1$ \\
\hline 11 & MaxBPS & Max data throughput & UInt32 & 4 & $004 \mathrm{~B} 0: 1200$ \\
\hline 12 & MaxCDev & Max Connected Devices & UInt16 & 2 & $01: 1$ \\
\hline 13 & MaxRDey & Max Registered Devices & UInt16 & 2 & $01: 1$ \\
\hline 14 & Encrypt & Encryption & UInt16 & 1 & $00: 0$ \\
\hline 15 & Authent & Authentication & Boolean & 1 & $0: 0$ \\
\hline 16 & MinKeyL & Min Key Length & UInd6 & 2 & $00: 0$ \\
\hline 17 & MaxKeyl & Mar Key Length & UInt16 & 2 & $00: 0$ \\
\hline is & MaxSDU & Max SDU Size & UInt16 & 2 & $01: 1$ \\
\hline 19 & MinALat & Min Access Latency & Ulnt32 & 4 & $0005: 5$ \\
\hline 20 & MinTLat & Min Transmit Latency & UInt32 & 4 & $0005: 5$ \\
\hline 21 & MaxXact & Max Simultaneous Trasactions & UInts & 1 & $1: 1$ \\
\hline 22 & Baterny & Device is battery powered & UInts & 1 & $1: 1$ \\
\hline 23 & Version & Version \# & UInt16 & 2 & $00: 0$ \\
\hline 24 & MaxRetry & Max Retries before Disconnect & UInt\$ & 1 & $05: 5$ \\
\hline 41 & Baud & Baud rate & UInt32 & 4 & $002550: 9600$ \\
\hline 42 & DataBits & Data bits & UInis & 1 & $8: 8$ \\
\hline 43 & Parity & Mark Parity & UInts & 1 & $0: 0$ \\
\hline 44 & StopBit & Stop bit & UInt8 & 1 & $1: 1$ \\
\hline 45 & Terminator & Terminator & UInts & 1 & $0: 0$ \\
\hline null & Checksum & Checksum & null & 2 & FC 1B: 64539 \\
\hline \multicolumn{6}{|c|}{ Close } \\
\hline
\end{tabular}

Tabela A.9 - PHY TEDS IEEE1451.2 adaptado de IEEE P1451.2TM/D21 (não publicado).

Table 2- PHY TEDS data block

\begin{tabular}{|c|l|l|c|c|l|}
\hline $\begin{array}{c}\text { Field } \\
\text { Type }\end{array}$ & Field & Description & Type & \multicolumn{2}{|c|}{ Contents } \\
octets
\end{tabular}




\section{Anexo I - PHY TEDS - IEEE1451.5}

Tabela A.10 - PHY TEDS IEEE1451.5 (IEEE, 2007b).

Table 2-PHY TEDS data block

\begin{tabular}{|c|c|c|c|c|c|}
\hline $\begin{array}{l}\text { Field } \\
\text { type }\end{array}$ & Field name & Deseription & Type & $\begin{array}{c}N \\
\text { octets }\end{array}$ & Contents \\
\hline- & & TEDS length & Ulnt32 & 4 & \\
\hline 3 & TEDSID & $\begin{array}{l}\text { TEDS Identification } \\
\text { Header }\end{array}$ & Ulnt8 & 4 & \\
\hline $4-9$ & & Reserved & & & \\
\hline 10 & Radio & Radio Type & Ulnt8 & 1 & See Table 4 \\
\hline 11 & MaxBPS & Max data throughput & UInt32 & 4 & Bits per second \\
\hline 12 & MaxCDev & $\begin{array}{l}\text { Max Connected } \\
\text { Devices }\end{array}$ & Ulnt16 & 2 & $\begin{array}{l}\text { Maximum number of devices that } \\
\text { may be simultaneously operational } \\
\text { with this device }\end{array}$ \\
\hline 13 & MaxRDev & $\begin{array}{l}\text { Max Registered } \\
\text { Devices }\end{array}$ & Ulnt16 & 2 & $\begin{array}{l}\text { Maximum number of devices that } \\
\text { may be simultaneously registered } \\
\text { with this device }\end{array}$ \\
\hline 14 & Encrypt & Encryption & UInt16 & 2 & Encryption type and key length \\
\hline 15 & Authent & Authentication & Boolean & 1 & $\begin{array}{l}\text { TRUE - Authentication supported } \\
\text { FALSE - not supported }\end{array}$ \\
\hline 16 & MinKeyL & Min Key Length & Ulnt16 & 2 & $\begin{array}{l}\text { Minimum key length for security } \\
\text { functions }\end{array}$ \\
\hline 17 & MaxKeyL & Max Key Length & Ulnt16 & 2 & $\begin{array}{l}\text { Maximum key length for security } \\
\text { functions }\end{array}$ \\
\hline 18 & MaxSDU & Max SDU Size & Ulnt 16 & 2 & $\begin{array}{l}\text { Maximum payload size for transfer } \\
\text { between devices }\end{array}$ \\
\hline 19 & MinAL_at & Min Access Latency & Ulnt32 & 4 & $\begin{array}{l}\text { Minimum period for initiating a first } \\
\text { transmission to an unconnected } \\
\text { device }\end{array}$ \\
\hline 20 & MinTLat & Min Transmit Latency & Ulnt32 & 4 & $\begin{array}{l}\text { The period for transmitting an } \mathrm{N} \text { - } \\
\text { octet payload, where } \mathrm{N} \text { is the } \\
\text { minimum payload size }\end{array}$ \\
\hline 21 & MaxXact & $\begin{array}{l}\text { Max Simultancous } \\
\text { Transactions }\end{array}$ & Ulnt8 & 1 & $\begin{array}{l}\text { Maximum number of queued, } \\
\text { uncompleted commands }\end{array}$ \\
\hline 22 & Battery & $\begin{array}{l}\text { Device is battery } \\
\text { powered }\end{array}$ & Ulnt 8 & 1 & $\begin{array}{l}\text { A nonzero value indicates battery } \\
\text { power }\end{array}$ \\
\hline 23 & RadioVer & Radio Version If & Ulnt 16 & 2 & A zero value indicates unknown \\
\hline 24 & MaxRetry & $\begin{array}{l}\text { Maximum Retries } \\
\text { before Disconnect }\end{array}$ & Uint 16 & 2 & $\begin{array}{l}\text { A zero value indicates never } \\
\text { disconnect }\end{array}$ \\
\hline $25-31$ & & Reserved & & & \\
\hline $32-41$ & & $\begin{array}{l}\text { Bluctooth Protocol } \\
\text { Specific }\end{array}$ & & & See Table 7 \\
\hline $42-47$ & & Reserved & & & \\
\hline $48-54$ & & 802-11 Radio Specific & & & \\
\hline $55-63$ & & Reserved & & & \\
\hline $64-80$ & & $\begin{array}{l}\text { ZigBee Protocol } \\
\text { Specific }\end{array}$ & & & \\
\hline $81-95$ & & Reserved & & & \\
\hline $96-103$ & & $\begin{array}{l}\text { 6LoWWPAN Protocol } \\
\text { Specific }\end{array}$ & & & \\
\hline $104-127$ & & Reserved & & & \\
\hline $128-255$ & & Open to manuficturers & & & \\
\hline$=$ & & Checksum & Ulnt16 & 2 & \\
\hline
\end{tabular}

